\title{
Coulomb scattering in the massless Nelson model II. Regularity of ground states
}

\author{
Wojciech Dybalski \\ Zentrum Mathematik, Technische Universität München, \\ E-mail: dybalski@ma.tum.de \\ Alessandro Pizzo \\ Dipartimento di Matematica, Università di Roma "Tor Vergata" \\ E-mail: pizzo@axp.mat.uniroma2.it
}

\begin{abstract}
For the massless Nelson model we provide detailed information about the dependence of the normalized ground states $\breve{\psi}_{P, \sigma}$ of the fiber single-electron Hamiltonians $H_{P, \sigma}$ on the total momentum $P$ and the infrared cut-off $\sigma$. This information is obtained with the help of the iterative analytic perturbation theory. In particular, we derive bounds of the form

$$
\left\|\partial_{P_{i}} \breve{\psi}_{P, \sigma}\right\|,\left\|\partial_{P_{i}} \partial_{P_{j}} \check{\psi}_{P, \sigma}\right\| \leq \frac{c}{\sigma^{\delta_{\lambda_{0}}}},
$$

for some constant $c$ and a function of the maximal admissible coupling constant $\lambda_{0} \mapsto \delta_{\lambda_{0}}$ s.t. $\lim _{\lambda_{0} \rightarrow 0} \delta_{\lambda_{0}}=0$. These results hold both in the infrared-regular and infrared-singular case. They are exploited in part I of this series to construct the two-electron scattering states in the infrared-regular massless Nelson model (in the absence of an infrared cut-off) along the lines of Haag-Ruelle scattering theory. They should also be relevant for the problem of scattering of two infraparticles in the infrared-singular Nelson model, whose solution is the goal of this series of papers. Although a part of a larger investigation, the present work is written in a self-contained fashion.
\end{abstract}

\section{Introduction}

In spite of tremendous progress in the mathematical description of scattering of light and matter in the framework of non-relativistic QED [7, 9, 30, 22, 32, 10], processes involving several massive particles ('electrons') remained untreated. This important gap in our understanding can be traced back to serious conceptual and technical difficulties arising at the multi-electron level. On the conceptual side, it is essential to follow the lines of relativistic scattering theory [26, 31, 11], in spite of the fact that the 'bare electron', appearing in the model Hamiltonian, is a non-relativistic quantum-mechanical particle. In the presence of a fixed infrared cut-off [18] and for massive photons [2] a suitable variant of Haag-Ruelle scattering theory was found long time ago. However, only in our recent paper [14], this framework was generalized to theories with non-trivial infrared structure. In [14] we constructed scattering states of two electrons in the infrared-regular massless Nelson model (without an infrared cut-off) and proved their tensor product structure. This result relies on detailed spectral properties of the ground states of the single-particle fiber Hamiltonians which encode localization of 
physical electrons in space. In the present paper and in [15] we prove these properties using iterative analytic perturbation theory $[29,27,8,3]$. Hence, our spectral results here derived are the basis for the rigorous scattering theory of non-relativistic quantum particles interacting with a quantized massless boson field. While the interaction mediated by low energy (massless) bosons still requires some regularization in [14], no infrared cut-off is present.

Let $H$ be the Hamiltonian of the massless Nelson model and $H_{P}$ be the usual fiber Hamiltonians at fixed total momentum $P$ defined in (2.2) below. The analysis of the spectrum of $H_{P}$ was initiated in $[17,19]$ and advanced in [29], where the iterative analytic perturbation theory was developed. Interesting results on the spectrum of the Nelson model with a slightly different form factor were also obtained in [1] by means of different methods. The results of the present paper go much beyond the existing applications of the iterative analytic perturbation theory. In order to elucidate these improvements let us now discuss our findings in non-technical terms: Let $H_{P, \sigma}$ be the fiber Hamiltonians with an infrared cut-off $\sigma>0$ which have normalized ground state vectors $\breve{\psi}_{P, \sigma}$ (with phases fixed in Definition 5.3) corresponding to isolated simple eigenvalues $E_{P, \sigma}$. We recall that in the case of the physical electrodynamic interaction (morally corresponding to the form factor (2.3) with $\bar{\alpha}=0$ ) the vector $\breve{\psi}_{P, \sigma}$ tends weakly to zero as $\sigma \rightarrow 0$. In all cases $\bar{\alpha} \geq 0$, the behaviour of the derivatives $\partial_{P}^{\beta} \breve{\psi}_{P, \sigma}$ in the limit $\sigma \rightarrow 0$ carries information about the localization of the electron in space and therefore is of central importance for the problem of scattering of several particles. In more technical terms, these derivatives enter the proof of existence of scattering states via the non-stationary phase method [14].

The analysis of these derivatives, given in the present paper, proceeds as follows: With the help of the unitary dressing transformation $W_{P, \sigma}$, defined in (3.14) below, we obtain the following formula

$$
\partial_{P} \check{\psi}_{P, \sigma}=W_{P, \sigma}^{*} \frac{1}{H_{P, \sigma}^{W}-E_{P, \sigma}}\left(\Gamma_{P, \sigma}\right)_{i} \check{\phi}_{P, \sigma},
$$

where $H_{P, \sigma}^{W}=W_{P, \sigma} H_{P, \sigma} W_{P, \sigma}^{*}$ and $\check{\phi}_{P, \sigma}=W_{P, \sigma} \breve{\psi}_{P, \sigma}$. The expression $\Gamma_{P, \sigma}$, defined by the formula

$$
\Gamma_{P, \sigma}=\nabla E_{P, \sigma}-W_{P, \sigma}\left(P-P_{\mathrm{f}}\right) W_{P, \sigma}^{*}
$$

where $P_{\mathrm{f}}$ is the free photon momentum operator, has the property $\left\langle\check{\phi}_{P, \sigma},\left(\Gamma_{P, \sigma}\right)_{i} \breve{\phi}_{P, \sigma}\right\rangle=0$ due to the HellmanFeynman formula. The behaviour of the r.h.s. of (1.1) as a function of $\sigma$ can be controlled with the help of iterative analytic perturbation theory, and we obtain in Corollary 5.7 that

$$
\left\|\frac{1}{H_{P, \sigma}^{W}-E_{P, \sigma}}\left(\Gamma_{P, \sigma}\right)_{i} \check{\phi}_{P, \sigma}\right\| \leq \frac{c}{\sigma^{\delta_{\lambda_{0}}}},
$$

where $c$ is independent of $\sigma$ and $\lambda_{0} \mapsto \delta_{\lambda_{0}}$ tends to zero as a function of the maximal admissible coupling constant. Clearly, we have an analogous bound on the first derivative of the vector

$$
\left\|\partial_{P_{i}} \breve{\psi}_{P, \sigma}\right\| \leq \frac{c}{\sigma^{\delta_{\lambda_{0}}}} .
$$

This preliminary result is within reach of existing applications of iterative analytic perturbation theory (see e.g. $[27,23]$ for estimates comparable to (1.3) in different models). However, it is not sufficient for constructing scattering states of two electrons. For this purpose the second derivative is needed.

The analysis of the second derivative of $\breve{\psi}_{P, \sigma}$ is considerably more difficult. It starts with a derivation of a formula which has the following form

$$
\begin{aligned}
\partial_{P_{i}} \partial_{P_{i^{\prime}}} \check{\psi}_{P, \sigma}= & W_{P, \sigma}^{*}\left(\frac{Q_{P, \sigma}^{\perp}}{H_{P, \sigma}^{W}-E_{P, \sigma}}\left(\Gamma_{P, \sigma}\right)_{i^{\prime}} \frac{1}{H_{P, \sigma}^{W}-E_{P, \sigma}}\left(\Gamma_{P, \sigma}\right)_{i} \check{\phi}_{P, \sigma}+\left\{i \leftrightarrow i^{\prime}\right\}\right) \\
& -W_{P, \sigma}^{*} \check{\phi}_{P, \sigma}\left\langle\check{\phi}_{P, \sigma},\left(\Gamma_{P, \sigma}\right)_{i^{\prime}} \frac{1}{\left(H_{P, \sigma}^{W}-E_{P, \sigma}\right)^{2}}\left(\Gamma_{P, \sigma}\right)_{i} \check{\phi}_{P, \sigma}\right\rangle,
\end{aligned}
$$


where $Q_{P, \sigma}=\left|\check{\phi}_{P, \sigma}\right\rangle\left\langle\check{\phi}_{P, \sigma}\right|$. The last term on the r.h.s. of (1.5) can be estimated using (1.3). To control the first two terms we use iterative analytic perturbation theory to prove the following bound (cf. Corollary 5.7)

$$
\left\|\frac{Q_{P, \sigma}^{\perp}}{H_{P, \sigma}^{W}-E_{P, \sigma}}\left(\Gamma_{P, \sigma}\right)_{i} \frac{1}{H_{P, \sigma}^{W}-E_{P, \sigma}}\left(\Gamma_{P, \sigma}\right)_{i^{\prime}} \check{\phi}_{P, \sigma}\right\| \leq \frac{c}{\sigma^{2 \delta_{\lambda_{0}}}},
$$

and consequently we obtain:

$$
\left\|\partial_{P_{i}} \partial_{P_{i^{\prime}}} \breve{\psi}_{P, \sigma}\right\| \leq \frac{c}{\sigma^{2 \delta_{\lambda_{0}}}} .
$$

We emphasize that the proof of (1.6), which is the main technical result of this paper, is much more involved than the proof of (1.3) and other applications of the iterative analytic perturbation theory available in the literature. It requires extensive use of direct integral representations (cf. Subsection 3.4) and application of novel maximal modulus principle arguments (cf. Subsection 3.5). Another complication comes from unexpected singular terms, which prevent simple power-counting arguments (we refer e.g. to $B^{*}\left(\left.G\right|_{n} ^{n-1}\right) \phi_{P, n-1}$ and $b^{*}\left(\left.\left.\mathcal{I}\right|_{n} ^{n-1} \cdot g\right|_{n} ^{n-1}\right) \phi_{P, n-1}$ in (4.201)). A more thorough discussion of these new ingredients is presented in Subsection 4.1 .

We stress that our main spectral results reported in (1.4) and (1.7) hold also when $\bar{\alpha}=0$ in the form factor that models the interaction (see (2.3)). These results are surely needed for the construction of scattering of several infraparticles in the infrared-singular massless Nelson model, which is the goal of this series of papers. However, differently from the control of the effective dynamics up to some time scale related to the coupling constant (cf [4]), the construction of scattering states for infraparticles requires a nontrivial control of the clouds of asymptotic real photons "attached" to the charged particles emerging out of the scattering; see [30], [8] for the case of a single infraparticle. This control poses new conceptual problems in the case of many electrons. An overview of them can be found in [12] along with clear-cut mathematical conjectures, with the aim to provide a consistent LSZ scattering theory that corrects the accepted (in the physics community) but incomplete ansatz provided by Fadeev and Kulish and based on a modified asymptotic dynamics a la Dollard.

We hope that the progress from the present paper will enable other interesting investigations of regularity of the ground state vectors in models of non-relativistic QED. For example, in order to improve the control of the $P$ - dependence of $\check{\phi}_{P, \sigma}$, uniformly in $\sigma$, our results naturally come into play. We recall that the Hölder regularity in $P$, uniformly in $\sigma$, of $\breve{\phi}_{P, \sigma}$ is a crucial ingredient in the analysis of the effective dynamics of an electron coupled to an external potential and interacting with the quantized radiation; see [4]. In fact, by expressing the 1.h.s of (1.1) in terms of $\breve{\phi}_{P, \sigma}$, we obtain

$$
\partial_{P_{i}} \check{\phi}_{P, \sigma}=\frac{1}{H_{P, \sigma}^{W}-E_{P, \sigma}}\left(\Gamma_{P, \sigma}\right)_{i} \check{\phi}_{P, \sigma}+\left(\int d^{3} k f_{P, \sigma}^{(i)}(k) b(k)-h . c .\right) \check{\phi}_{P, \sigma},
$$

where $b(k)$ is the (improper) annihilation operator and $f_{P, \sigma}^{(i)}$ is a concrete function which behaves as $|k|^{-3 / 2}$ for $\sigma \leq|k| \leq 1$. We conjecture that this latter singularity is compensated by the the first term on the r.h.s. of (1.8) and altogether $\left\|\partial_{P_{i}} \breve{\phi}_{P, \sigma}\right\| \leq c$ holds uniformly in $\sigma$. Such a bound would require certain refinements of estimate (1.3), with more explicit control of the singular behaviour. We will not pursue this direction here, but we believe that it is within reach of the methods developed in this paper.

Our paper is organized as follows: In Section 2 we state our main result about regularity of the ground states and discuss a corollary about regularity of wave functions, proven in [15]. In Section 3 we include some preliminaries on Hamiltonians and their ground states, the shift of the infrared cut-off, standard resolvent bounds, direct integral representations and the maximal modulus principle. Section 4 is the core of the paper, it includes the inductive proof of estimate (1.6). In Section 5 we derive formulas for the derivatives of ground states and ground state energies, including (1.1), (1.5), and conclude the proof of our main result (Theorem 2.1), 
including estimates (1.4), (1.7). In the main part of the paper, outlined above, the focus is on new results. In the appendices we typically reestablish (under our assumptions and for the present model) relevant results from the existing literature. Thereby the paper is essentially self-contained.

Acknowledgment: A.P. thanks the Hausdorff Research Institute of Mathematics, Bonn, for hospitality. A.P. was supported by the NSF grant \#DMS-0905988. A.P. acknowledges the MIUR Excellence Department Project awarded to the Department of Mathematics, University of Rome Tor Vergata, CUP E83C18000100006.

W.D. thanks the University of California Davis and the Hausdorff Research Institute for Mathematics, Bonn, for hospitality. W.D. is supported by the German Research Foundation (DFG) within the grants SP181/25-2, DY107/1-1, DY107/2-1. Moreover, he would like to acknowledge the support of the Danish Council for Independent Research, grant no. 09-065927 "Mathematical Physics", and of the Lundbeck Foundation. Last but not least, W.D. would like to thank his wife Jasmin for her continuing support in the course of this long-term project.

\section{Results}

In this section we state the main results of this paper which were announced already in Section 1.2 of [14].

Let $\mathfrak{h}_{\mathrm{fi}}=L^{2}\left(\mathbb{R}^{3}, d^{3} k\right)$ be the single-photon subspace in the fiber picture. Let $\mathcal{F}:=\Gamma\left(\mathfrak{h}_{\mathrm{fi}}\right)$ be the symmetric Fock space over $\mathfrak{h}_{\mathrm{fi}}$ and let us denote the corresponding (improper) creation and annihilation operators by $b^{*}(k)$ and $b(k)$. The free fiber Hamiltonian of the massless Nelson model at a fixed total momentum $P$ has the form

$$
H_{P, \text { free }}:=\frac{1}{2}\left(P-P_{\mathrm{f}}\right)^{2}+H_{\mathrm{f}}, \text { where } H_{\mathrm{f}}:=\int d^{3} k|k| b^{*}(k) b(k), \quad P_{\mathrm{f}}:=\int d^{3} k k b^{*}(k) b(k),
$$

whereas the interacting fiber Hamiltonian is given by

$$
H_{P}:=\frac{1}{2}\left(P-P_{\mathrm{f}}\right)^{2}+H_{\mathrm{f}}+\int d^{3} k v_{\bar{\alpha}}(k)\left(b(k)+b^{*}(k)\right) .
$$

To define the form factor $v_{\bar{\alpha}}$ we need to introduce some notation: Let $\mathcal{B}_{r}$ be the open ball of radius $r>0$ centered at zero. Let $\chi_{r} \in C_{0}^{\infty}\left(\mathbb{R}^{3}\right)$ be a function which is rotationally invariant, non-increasing in the radial direction, supported in $\mathcal{B}_{r}$ and equal to one on $\mathcal{B}_{\left(1-\varepsilon_{0}\right) r}$, for $0<\varepsilon_{0}<1$. Let $0<|\lambda| \leq 1$ be the coupling constant and $\kappa>0$ the ultraviolet cut-off which is kept fixed in our investigation. For simplicity of the presentation we will set $\kappa=1$. The form-factor is given by

$$
v_{\bar{\alpha}}(k):=\lambda \frac{\chi_{\kappa}(k)|k|^{\bar{\alpha}}}{(2|k|)^{\frac{1}{2}}}
$$

where $\frac{1}{2} \geq \bar{\alpha} \geq 0$ is a parameter which controls the infrared behaviour of the system. (For $\bar{\alpha}=0$ we say that the model is infrared singular, for $\bar{\alpha}>0$ that it is infrared regular). This parameter is kept fixed.

An important role in the spectral analysis of the Hamiltonians $H_{P}$ is played by their counterparts $H_{P, \sigma}$ with an infrared cut-off $\sigma \in(0, \kappa]$. They are given by

$$
H_{P, \sigma}:=\frac{1}{2}\left(P-P_{\mathrm{f}}\right)^{2}+H_{\mathrm{f}}+\int d^{3} k v_{\bar{\alpha}}^{\sigma}(k)\left(b(k)+b^{*}(k)\right)
$$

The form-factor $v_{\bar{\alpha}}^{\sigma}$, which carries information both about the (sharp) infrared cut-off $\sigma$ and the (smooth) ultraviolet cut-off $\kappa$, is defined as

$$
v_{\bar{\alpha}}^{\sigma}(k):=\lambda \frac{\chi_{[\sigma, \kappa)}(k)|k|^{\bar{\alpha}}}{(2|k|)^{\frac{1}{2}}}
$$


where $\chi_{[\sigma, \kappa)}(k):=\mathbf{1}_{\mathcal{B}_{\sigma}^{\prime}}(k) \chi_{\kappa}(k), \mathcal{B}_{r}:=\left\{k \in \mathbb{R}^{3}|| k \mid<r\right\}$ and $\mathcal{B}_{r}^{\prime}=\mathbb{R}^{3} \backslash \mathcal{B}_{r}$. By the Kato-Rellich theorem, $H_{P}$ and $H_{P, \sigma}$ are self-adjoint operators on $D\left(H_{P, \text { free }}\right)$.

As our analysis concerns the bottom of the spectrum of the fiber Hamiltonians, let us define

$$
E_{P}:=\inf \sigma\left(H_{P}\right), \quad E_{P, \sigma}:=\inf \sigma\left(H_{P, \sigma}\right),
$$

where $\sigma$ denotes the spectrum. Since the model is non-relativistic, we are interested in small values of the total momentum $P$ for which the electron moves slower than the photons. For this reason we consider $P$ from the set

$$
S:=\left\{P \in \mathbb{R}^{3}|| P \mid<P_{\max }\right\}
$$

for $P_{\max }=1 / 3$. Since we work in the weak coupling regime, we fix some sufficiently small $\lambda_{0}>0$, specified in Theorem 2.1, and restrict attention to $|\lambda| \in\left(0, \lambda_{0}\right]$.

In the following theorem, which is our main result, we collect the results concerning $E_{P}, E_{P, \sigma}$ and the corresponding ground state vector $\breve{\psi}_{P, \sigma}$ which are needed in the scattering theory of two electrons in [14].

Theorem 2.1. Fix $0 \leq \bar{\alpha} \leq 1 / 2$ and let $P_{\max }=1 / 3$. Then there exists $\lambda_{0}>0$ and $\kappa \geq \kappa_{\lambda_{0}}>0$ s.t. for all $|\lambda| \in\left(0, \lambda_{0}\right]$ and $P \in S:=\mathcal{B}_{P_{\max }}$ the following statements hold:

(a) For $\sigma \in\left(0, \kappa_{\lambda_{0}}\right], E_{P, \sigma}$ is a simple eigenvalue corresponding to a normalized eigenvector $\breve{\psi}_{P, \sigma}$, whose phase is fixed in Definition 5.3 below. $S \ni P \mapsto E_{P, \sigma}$ is analytic and strictly convex, for all $\sigma \in\left(0, \kappa_{\lambda_{0}}\right]$. Moreover, for some $0<\delta_{\lambda_{0}}<1 / 4$, specified below

$$
\begin{aligned}
& \left|\partial_{P}^{\beta_{1}} E_{P, \sigma}\right| \leq c, \quad\left|\partial_{P}^{\beta_{2}} E_{P, \sigma}\right| \leq c, \quad\left|\partial_{P}^{\beta_{3}} E_{P, \sigma}\right| \leq c / \sigma^{\delta_{\lambda_{0}}} \\
& \left\|\partial_{P}^{\beta} \breve{\psi}_{P, \sigma}\right\| \leq c / \sigma^{\delta_{\lambda_{0}}}
\end{aligned}
$$

for multiindices $\beta_{i}, \beta$ s.t. $\left|\beta_{i}\right|=i$ and $0<|\beta| \leq 2$.

(b) For $\sigma \in\left(0, \kappa_{\lambda_{0}}\right]$ the estimate

$$
\left|E_{P}-E_{P, \sigma}\right| \leq c \sigma
$$

holds true. Moreover, $S \ni P \mapsto E_{P}$ is twice continuously differentiable and strictly convex.

(c) For $\bar{\alpha}>0, E_{P}$ is an eigenvalue corresponding to a normalized eigenvector $\check{\psi}_{P}$. Moreover, for a suitable choice of the phase of $\check{\psi}_{P}$ and $\sigma \in\left(0, \kappa_{\lambda_{0}}\right]$

$$
\left\|\check{\psi}_{P}-\breve{\psi}_{P, \sigma}\right\| \leq c(\bar{\alpha})^{-1} \sigma^{\bar{\alpha}} .
$$

The constant $c$ above is independent of $\sigma, P, \lambda, \bar{\alpha}$ within the assumed restrictions. Clearly, all statements above remain true after replacing $\lambda_{0}$ by some $\tilde{\lambda}_{0} \in\left(0, \lambda_{0}\right]$. The resulting function $\tilde{\lambda}_{0} \mapsto \delta_{\tilde{\lambda}_{0}}$ can be chosen s.t. $\lim _{\tilde{\lambda}_{0} \rightarrow 0} \delta_{\tilde{\lambda}_{0}}=0$.

We give the proof of this theorem in the last part of Section 5. The most innovative statements are the bound on the third derivative of $E_{P, \sigma}$ in (2.8) and on the first and second derivative of $\breve{\psi}_{P, \sigma}$ in (2.9). Other items have already been established in the Nelson model or in similar models: The fact that $S \ni P \mapsto E_{P}, S \ni P \mapsto E_{P, \sigma}$ are twice continuously differentiable and convex has been shown in non-relativistic and semi-relativistic QED in $[23,27,5]$ and in the Nelson model with a slightly different form-factor in [1]. The bound in (2.10) can be extracted from [29]. The first statement in (a) has been established already in [19]. Part (c) is implicit in [29].

It turns out that the regularity of the vector $\breve{\psi}_{P, \sigma}$, established in Theorem 2.1, is not sufficient for scattering theory of two electrons. One also needs analogous bounds for the wave functions $\left\{f_{P, \sigma}^{q}\right\}_{q \in \mathbb{N}_{0}}$ of $\breve{\psi}_{P, \sigma}$. Although these estimates are thoroughly discussed and proven in a companion paper [15], we state them here as they constitute an interesting corollary of Theorem 2.1. 
Clearly, each $f_{P, \sigma}^{q}$ is a square-integrable function symmetric in $q$ variables from $\mathbb{R}^{3}$. We introduce the following auxiliary functions:

$$
g_{\sigma}^{q}\left(k_{1}, \ldots, k_{q}\right):=\prod_{i=1}^{q} \frac{c \lambda \chi_{\left[\sigma, \kappa_{*}\right)}\left(k_{i}\right)\left|k_{i}\right|^{\bar{\alpha}}}{\left|k_{i}\right|^{3 / 2}}, \quad \kappa_{*}:=\left(1-\varepsilon_{0}\right)^{-1} \kappa,
$$

for some $0<\varepsilon_{0}<1$, where $c$ is a positive constant independent of $q, \sigma, P$ and $\lambda$ within the restrictions specified above. Next, we introduce the notation

$$
\mathcal{A}_{r_{1}, r_{2}}:=\left\{k \in \mathbb{R}^{3}\left|r_{1}<\right| k \mid<r_{2}\right\},
$$

where $0 \leq r_{1}<r_{2}$. Now we are ready to state the required properties of the functions $f_{P, \sigma}^{q}$ :

Theorem 2.2. Fix $0 \leq \bar{\alpha} \leq 1 / 2$ and let $P_{\max }=1 / 3$. Then there exists $\lambda_{0}>0$ and $\kappa>\kappa_{\lambda_{0}}>0$ s.t. for all $P \in S=\mathcal{B}_{P_{\max }}, \lambda \in\left(0, \lambda_{0}\right]$ and $\sigma \in\left(0, \kappa_{\lambda_{0}}\right]$ there holds:

(a) Let $\left\{f_{P, \sigma}^{q}\right\}_{q \in \mathbb{N}_{0}}$ be the q-particle components of $\breve{\psi}_{P, \sigma}$ and let $\overline{\mathcal{A}}_{\sigma, \kappa}^{\times q}$ be defined as the Cartesian product of $q$ copies of the closure of the set $\mathcal{A}_{\sigma, \kappa}$ introduced in (2.13). Then, for any $P \in S$, the function $f_{P, \sigma}^{q}$ is supported in $\overline{\mathcal{A}}_{\sigma, \kappa}^{\times q}$.

(b) The function

$$
S \times \mathcal{A}_{\sigma, \infty}^{\times q} \ni\left(P ; k_{1}, \ldots, k_{q}\right) \mapsto f_{P, \sigma}^{q}\left(k_{1}, \ldots, k_{q}\right)
$$

is twice continuously differentiable and extends by continuity, together with its derivatives, to the set $S \times \overline{\mathcal{A}}_{\sigma, \infty}^{\times q}$.

(c) For any multiindex $\beta, 0 \leq|\beta| \leq 2$, the function (2.14) satisfies

$$
\begin{aligned}
\left|\partial_{k_{l}}^{\beta} f_{P, \sigma}^{q}\left(k_{1}, \ldots, k_{q}\right)\right| & \leq \frac{1}{\sqrt{q !}}\left|k_{l}\right|^{-|\beta|} g_{\sigma}^{q}\left(k_{1}, \ldots, k_{q}\right), \\
\left|\partial_{P}^{\beta} f_{P, \sigma}^{q}\left(k_{1}, \ldots, k_{q}\right)\right| & \leq \frac{1}{\sqrt{q !}}\left(\frac{1}{\sigma^{\delta_{\lambda_{0}}}}\right)^{|\beta|} g_{\sigma}^{q}\left(k_{1}, \ldots, k_{q}\right), \\
\left|\partial_{P^{i}} \partial_{k_{l}^{i}} f_{P, \sigma}^{q}\left(k_{1}, \ldots, k_{q}\right)\right| & \leq \frac{1}{\sqrt{q !}} \frac{1}{\sigma^{\delta_{\lambda_{0}}}}\left|k_{l}\right|^{-1} g_{\sigma}^{q}\left(k_{1}, \ldots, k_{q}\right),
\end{aligned}
$$

where the function $\tilde{\lambda}_{0} \mapsto \delta_{\tilde{\lambda}_{0}}$ has the properties specified in Theorem 2.1 and $g_{\sigma}^{q}$ is defined in (2.12).

We give the proof of Theorem 2.2 in a companion paper [15]. Parts (a), (b) and estimate (2.15) in (c) can be extracted from $[17,19,18]$ or proven using the methods from these papers. Estimate $(2.16)$ for $|\beta|=1$ and (2.17) rely on the standard formula for the ground state wave functions from [17, 19] combined with the spectral ingredient (1.3). This latter ingredient is relatively well established in models of non-relativistic QED [23, 27] and proven for the Nelson model in the present paper (see Theorem 4.1 below). The key new result in Theorem 2.2 is the bound (2.16) for $|\beta|=2$ which requires a novel, 'infrared safe' formula for the wave functions, derived in [15] by solving a non-commutative recurrence relation. This new formula is then combined with the spectral ingredient (1.6) which is the main technical result of the present paper (see Theorem 4.3 below).

The infrared regular case (i.e. $0<\bar{\alpha} \leq 1 / 2$ ) is meanwhile relatively well understood (see e.g. [24, 16]). It is likely that in this situation the results from Theorems 2.1, 2.2 could be strengthened and their proofs simplified. While this would suffice for scattering of two electrons in the infrared regular Nelson model, described in [14], it would not help to control collisions of two infraparticles, which is the goal of this project. In this context the 
iterative analytic perturbation theory, which we use and further develop in this paper, appears to be the optimal approach.

\section{Standing assumptions and conventions:}

1. We denote by $c, c^{\prime}, c^{\prime \prime}$ numerical constants which may change from line to line. These constants are universal in the sense that are independent of $\sigma, P, \lambda, \bar{\alpha}, \epsilon$, within the assumed restrictions.

2. Upper or lower indices $i, i^{\prime}, \hat{i}, \hat{i}^{\prime}=1,2,3$ denote components of vectors in $\mathbb{R}^{3}$.

3. We denote by $\left\langle\psi_{1}, \psi_{2}\right\rangle$ the scalar product of two vectors $\psi_{1}, \psi_{2}$ in a Hilbert space.

4. The contour integrals are oriented counterclockwise. Moreover, we use the convention

$$
-\frac{1}{2 \pi i} \oint_{\gamma} \equiv \oint_{\gamma}
$$

for a contour $\gamma$. With this convention $\oint_{\gamma} d z / z=-1$ if $\gamma$ is a circle centered at zero.

5. The symbol $\mathcal{B}_{r}$ denotes the set $\left\{k \in \mathbb{R}^{3}:|k|<r\right\}$.

6. $\chi_{r} \in C_{0}^{\infty}\left(\mathbb{R}^{3}\right)$ is a function which is rotationally invariant, non-increasing in the radial direction, supported in $\mathcal{B}_{r}$ and equal to one on $\mathcal{B}_{\left(1-\varepsilon_{0}\right) r}$, for some fixed $0<\varepsilon_{0}<1$.

7. The symbol $\int_{r_{1}}^{r_{2}} d^{3} k$ stands for $\int_{\mathcal{B}_{r_{2}} \backslash \mathcal{B}_{r_{1}}} d^{3} k$.

8. The symbol $\int_{r_{1}}^{r_{2}} d^{3} k$ stands for $\int_{\mathcal{B}_{r_{2}} \backslash \mathcal{B}_{r_{1}}} d^{3} k \chi_{\kappa}(k)|k|^{\bar{\alpha}}$, where $\kappa=1$ is the UV cut-off.

9. The symbol $\int_{r_{1}}^{r_{2}} d^{3} k$ stands for $\int_{\mathcal{B}_{r_{2}} \mid \mathcal{B}_{r_{1}}} d^{3} k \chi_{\kappa}(k)^{2}|k|^{2 \bar{\alpha}}$.

10. We set $\sigma_{n}:=\kappa \epsilon^{n}$, where $n \in \mathbb{N}_{0}$ and $0<\epsilon \leq \frac{1}{2}, \kappa=1$.

11. We set $\left.\mathcal{A}\right|_{n} ^{n-1}:=\mathcal{A}_{\sigma_{n}, \sigma_{n-1}}=\left\{k \in \mathbb{R}^{3}\left|\sigma_{n}<\right| k \mid<\sigma_{n-1}\right\}$.

12. The symbol $\hat{\int}_{\left.\left.\mathcal{A}\right|_{n} ^{n-1}\right)^{\times m}} d^{3 m} k$ stands for $\int_{\mathcal{B}_{\sigma_{n-1}} \mid \mathcal{B}_{\sigma_{n}}} d^{3} k_{1} \ldots \int_{\mathcal{B}_{\sigma_{n-1}} \mid \mathcal{B}_{\sigma_{n}}} d^{3} k_{m} \chi_{\kappa}\left(k_{1}\right)\left|k_{1}\right|^{\bar{\alpha}} \ldots \chi_{\kappa}\left(k_{m}\right)\left|k_{m}\right|^{\bar{\alpha}}$.

13. The symbol $\overline{\int \mathcal{A}}_{\left.n{ }_{n}^{n-1}\right)^{\times m}} d^{3 m} k$ stands for $\int_{\mathcal{B}_{\sigma_{n-1}} \backslash \mathcal{B}_{\sigma_{n}}} d^{3} k_{1} \cdots \int_{\mathcal{B}_{\sigma_{n-1}} \backslash \mathcal{B}_{\sigma_{n}}} d^{3} k_{m} \chi_{\kappa}\left(k_{1}\right)^{2}\left|k_{1}\right|^{2 \bar{\alpha}} \cdots \chi_{\kappa}\left(k_{m}\right)^{2}\left|k_{m}\right|^{2 \bar{\alpha}}$.

14. If $X$ is an element of a Banach space and $\|X\| \leq c Y$ for some $Y \in \mathbb{R}_{+}$then we write $X=O(Y)$. If, furthermore, $Y \leq c^{\prime} Z$ for some $Z \in \mathbb{R}_{+}$we will write $X=O(Y)=O(Z)$.

15. The symbol $\mathbb{N}_{0}$ stands for $\mathbb{N} \cup\{0\}$.

\section{Preliminaries}

\subsection{Hamiltonians and their ground states}

This subsection concerns the ground states of physical, transformed and intermediate Hamiltonians, defined by formulas (3.2), (3.16) and (3.17), respectively. One reason for introducing the transformed Hamiltonians is that their ground states exist in the limit $\sigma \rightarrow 0$ (cf. Corollary 5.1 below.)

First, we introduce a sequence of infrared cut-offs

$$
\sigma_{n}:=\kappa \epsilon^{n}, \quad n \in \mathbb{N}_{0}, \quad 0<\epsilon \leq \frac{1}{2}
$$


and the corresponding cut-off Hamiltonians at fixed total momentum and fixed ultraviolet cut-off $\kappa=1$, i.e.,

$$
H_{P, \sigma_{n}}=\frac{\left(P-P_{\mathrm{f}}\right)^{2}}{2}+\lambda \int_{\sigma_{n}}^{k} d^{3} k \frac{1}{\sqrt{2|k|}}\left\{b(k)+b^{*}(k)\right\}+H_{\mathrm{f}} .
$$

From now on, we will also write $H_{P, n}:=H_{P, \sigma_{n}}$ and $E_{P, n}:=E_{P, \sigma_{n}}$.

Remark 3.1. Since we work in the low-coupling regime, we will always assume that $|\lambda| \in\left(0, \lambda_{0}\right]$, where $\lambda_{0}$ is assumed to be sufficiently small though we will often omit this specification. Furthermore, we assume that $\epsilon \in\left(0, \epsilon_{0}\right]$ for some $0<\epsilon_{0} \leq 1 / 2$. The maximal values $\lambda_{0}, \epsilon_{0}$ will be reduced in the course of the argument, but will remain non-zero and depend only on universal constants. (Until the end of Section 3 we will have $\epsilon_{0}=1 / 2$, then this parameter will be decreased in Theorems 4.1 and 4.3). At any stage of the paper it is assumed that $\lambda_{0}$ and $\epsilon_{0}$ are chosen small enough so that all the preceding results hold.

For $n \in \mathbb{N}_{0}$, we define the bosonic Fock spaces for $m \leq n$

$$
\begin{aligned}
\mathcal{F} & :=\Gamma\left(L^{2}\left(\mathbb{R}^{3}, d^{3} k\right)\right), \\
\mathcal{F}_{n} & :=\Gamma\left(L^{2}\left(\mathbb{R}^{3} \backslash \mathcal{B}_{\sigma_{n}}, d^{3} k\right)\right), \\
\left.\mathcal{F}\right|_{n} ^{m} & :=\Gamma\left(L^{2}\left(\mathcal{B}_{\sigma_{m}} \backslash \mathcal{B}_{\sigma_{n}}, d^{3} k\right)\right) .
\end{aligned}
$$

In all these Fock spaces we shall use the same symbol $\Omega$ to denote the vacuum vector. Clearly, $\mathcal{F}_{n}=\mathcal{F}_{n-1} \otimes$ $\left.\mathcal{F}\right|_{n} ^{n-1}$. For a vector $\psi$ in $\mathcal{F}_{n-1}$ and an operator $O$ on $\mathcal{F}_{n-1}$ we shall use the same symbol to denote the vector $\psi \otimes \Omega$ in $\mathcal{F}_{n}$ and the operator $O \otimes 1_{\left.\mathcal{F}\right|_{n} ^{n-1}}$ on $\mathcal{F}_{n}$, respectively, where $1_{\left.\mathcal{F}\right|_{n} ^{n-1}}$ is the identity operator on $\left.\mathcal{F}\right|_{n} ^{n-1}$ (e.g., $\left.\int_{\kappa \epsilon^{n-1}}^{k} d^{3} k b(k) 1 \mathcal{F}_{n} \equiv \int_{\kappa \epsilon^{n-1}}^{k} d^{3} k b(k) \otimes 1_{\left.\mathcal{F}\right|_{n} ^{n-1}}\right)$. For a given operator $A$ on $\mathcal{F}$ we set $\|A\|_{\mathcal{F}_{n}}:=\left\|A 1 \mathcal{F}_{n}\right\|$. The Hamiltonians $H_{P, n}$ act on the Hilbert space $\mathcal{F}$.

Proposition 3.2, stated below, provides a basis for our investigation. This result is standard, but in the available literature (see $[29,6,8,27]$ ) there are differences in terms of models and ranges of parameters. Thus to make our work accessible to non-experts we provide a rather detailed proof in Appendix A. Some intermediate results from Appendix A will also be used at later stages of our discussion (e.g., estimate (A.26) enters into the proof of the resolvent bound (3.50) below).

Proposition 3.2. Let $|P| \leq P_{\max }=1 / 3,|\lambda| \in\left(0, \lambda_{0}\right], \epsilon \in(0,1 / 2]$ and $|\lambda| \leq \epsilon^{2}$. Then, for all $n \in \mathbb{N}_{0}$ the resolvent $\left(H_{P, n}-z\right)^{-1} 1 \mathcal{F}_{n}$ is well-defined in $(1 / 16) \sigma_{n} \leq\left|E_{P, n-1}-z\right| \leq(1 / 3) \sigma_{n}$. Furthermore, the Hamiltonian $H_{P, n} 1 \mathcal{F}_{n}$ has a unique (unnormalized) ground state vector $\psi_{P, n}$, and the corresponding ground state energy coincides with $E_{P, n}:=\inf \sigma\left(H_{P, n} 1 \mathcal{F}\right) . \psi_{P, n}$ is defined iteratively from $\psi_{P, 0} \equiv \Omega$ by the following relation valid for $n \geq 1$,

$$
\psi_{P, n}:=\oint_{\gamma_{P, n}} \frac{d w_{n}}{H_{P, n}-w_{n}} \psi_{P, n-1}, \quad \gamma_{P, n}:=\left\{w_{n} \in \mathbb{C}|| w_{n}-E_{P, n-1} \mid=\frac{\sigma_{n}}{3}\right\} .
$$

The following properties hold true: The function $S \ni P \mapsto E_{P, n}$ is analytic for any fixed $n \in \mathbb{N}_{0}$. Moreover,

$$
\begin{aligned}
& E_{P-k, n}-E_{P, n} \geq-\left(\frac{1}{3}+c|\lambda|\right)|k|, \quad k \in \mathbb{R}^{3}, \\
& \left|\nabla E_{P, n}\right| \leq \frac{1}{3}+c|\lambda|, \\
& E_{P, n} \leq E_{P, n-1}, \\
& \left|E_{P, n-1}-E_{P, n}\right| \leq c_{\Delta E} \lambda^{2} \sigma_{n-1}, \\
& \operatorname{Gap}\left(H_{P, n} 1 \mathcal{F}_{n}\right):=\inf _{\phi \perp \psi_{P, n}, \phi \in \mathcal{F}_{n}}\left\langle\frac{\phi}{\|\phi\|},\left(H_{P, n}-E_{P, n}\right) \frac{\phi}{\|\phi\|}\right\rangle \geq \frac{\sigma_{n}}{3}, \\
& \operatorname{Gap}\left(H_{P, n-1} 1 \mathcal{F}_{n}\right) \geq \frac{\sigma_{n}}{2},
\end{aligned}
$$

where $c, c_{\Delta E}$ are universal constants. 
Remark 3.3. It is clear from the above proposition that formula (3.6) and the estimate in (3.10) imply

$$
\psi_{P, n}=\oint_{\gamma_{P, n+1}} \frac{d w_{n+1}}{H_{P, n}-w_{n+1}} \psi_{P, n-1},
$$

as we modified the contour within the region of holomorphy. This variant will be more convenient for applications in this paper.

Next, we introduce the transformed Hamiltonians and some related formulas that will be needed in Lemma 3.12 and for the proof of Theorem 4.1. These definitions have been introduced in [29].

Definition 3.4. Let us introduce the Weyl operators:

$$
\begin{aligned}
& W_{P, n}:=\exp \left(-\lambda \int_{\sigma_{n}}^{K} \frac{d^{3} k}{\sqrt{2}|k|^{\frac{3}{2}} \alpha_{P, n}(\hat{k})}\left\{b(k)-b^{*}(k)\right\}\right), \\
& \widetilde{W}_{P, n}:=\exp \left(-\lambda \int_{\sigma_{n}}^{K} \frac{d^{3} k}{\sqrt{2}|k|^{\frac{3}{2}} \alpha_{P, n-1}(\hat{k})}\left\{b(k)-b^{*}(k)\right\}\right),
\end{aligned}
$$

where $\alpha_{P, n}(\hat{k}):=\left(1-\hat{k} \cdot \nabla E_{P, n}\right)$. The transformed Hamiltonians and the intermediate Hamiltonians are given by

$$
\begin{aligned}
& H_{P, n}^{W}:=W_{P, n} H_{P, n} W_{P, n}^{*}, \\
& \hat{H}_{P, n}^{W}:=\widetilde{W}_{P, n} H_{P, n} \widetilde{W}_{P, n}^{*}=\widetilde{W}_{P, n} W_{P, n}^{*} H_{P, n}^{W} W_{P, n} \widetilde{W}_{P, n}^{*},
\end{aligned}
$$

respectively. The equalities are meant on $D\left(H_{P, n}\right)=D\left(H_{P, \text { free }}\right)$ which is invariant under $W_{P, n}^{*}, \widetilde{W}_{P, n}^{*}$ [20]. (Equalities of unbounded operators in the remaining part of this section are meant on $D\left(H_{P \text {,free }}\right)$ unless stated otherwise).

Now we recall an iterative construction of ground states of the modified and transformed Hamiltonians from [29].

Corollary 3.5. Let $|P| \leq P_{\max }=1 / 3,|\lambda| \in\left(0, \lambda_{0}\right], \epsilon \in(0,1 / 2]$ and $|\lambda| \leq \epsilon^{2}$. The ground state vectors $\phi_{P, n}$ and $\hat{\phi}_{P, n}$ of the Hamiltonians $H_{P, n}^{W}$ and $\hat{H}_{P, n}^{W}$, respectively, are iteratively defined by

$$
\hat{\phi}_{P, n}:=\oint_{\gamma_{P, n+1}} \frac{d w_{n+1}}{\hat{H}_{P, n}^{W}-w_{n+1}} \phi_{P, n-1}, \quad \phi_{P, n}:=W_{P, n} \widetilde{W}_{P, n}^{*} \hat{\phi}_{P, n}, \quad \phi_{P, 0}:=\Omega
$$

They satisfy

$$
\left|\nabla E_{P, n-1}-\nabla E_{P, n}\right| \leq c_{1}\left[\lambda^{2} \sigma_{n-1}+\left\|\frac{\hat{\phi}_{P, n}}{\left\|\hat{\phi}_{P, n}\right\|}-\frac{\phi_{P, n-1}}{\left\|\phi_{P, n-1}\right\|}\right\|\right] .
$$

Proof. The contour integral in expression (3.18) is well defined by Proposition 3.2 and (3.13). Estimate (3.19), which follows from the proof of Lemma A.2 of [29], is verified in Lemma D.6.

\subsection{Shift of the cut-off from $\sigma_{n-1}$ to $\sigma_{n}$}

In Subsection 3.1 we studied how the ground state energy $E_{P, n}$ and the ground state vectors $\psi_{P, n}, \phi_{P, n}, \hat{\phi}_{P, n}$ behave under the change of the infrared cut-off by one step. In this subsection we determine the behaviour of various relevant operator quantities. The most important relations are (3.25), (3.36), (3.40). We start with a simple computational result. 
Lemma 3.6. [29] The transformed Hamiltonian has the form

$$
H_{P, n}^{W}=\frac{\Gamma_{P, n}^{2}}{2}+\int d^{3} k \alpha_{P, n}(k)|k| b^{*}(k) b(k)+c_{P}^{\sigma_{n}},
$$

where

$$
\begin{aligned}
\Gamma_{P, n} & :=\Pi_{P, n}-\frac{\left\langle W_{P, n} \psi_{P, n}, \Pi_{P, n} W_{P, n} \psi_{P, n}\right\rangle}{\left\langle\psi_{P, n}, \psi_{P, n}\right\rangle}=\nabla E_{P, n}-W_{P, n}\left(P-P_{\mathrm{f}}\right) W_{P, n}^{*}, \\
\Pi_{P, n} & :=P_{\mathrm{f}}-\lambda \int_{\sigma_{n}}^{\kappa} \frac{d^{3} k k}{\sqrt{2}|k|^{\frac{3}{2}} \alpha_{P, n}(\hat{k})}\left\{b(k)+b^{*}(k)\right\}, \\
c_{P}^{\sigma_{n}} & :=\frac{P^{2}}{2}-\frac{1}{2}\left(P-\nabla E_{P, n}\right)^{2}-\lambda^{2} \bar{\int}_{\sigma_{n}}^{\kappa} \frac{d^{3} k}{2|k|^{2} \alpha_{P, n}(\hat{k})} .
\end{aligned}
$$

Furthermore, $\left\langle\phi_{P, n}, \Gamma_{P, n} \phi_{P, n}\right\rangle=0$.

In Lemma 3.7 we will consider a new contour, namely

$$
\tilde{\gamma}_{P, n}:=\left\{z_{n} \in \mathbb{C} \mid \operatorname{Re} z_{n}=E_{P, n-1}+\frac{\sigma_{n}}{3}\right\},
$$

which appears in the statements of Theorems 4.1 and 4.3. We stress that while the former theorem could be proven with the standard contour $\gamma_{P, n}$, this does not seem to be the case for the latter theorem. The following expansion is used in the first step of the proof of Theorems 4.1 and 4.3 .

Lemma 3.7. [29] For $|P| \leq P_{\max }=1 / 3,|\lambda| \in\left(0, \lambda_{0}\right], \epsilon \in(0,1 / 2]$ and $|\lambda| \leq \epsilon^{4}$ the resolvent of the intermediate Hamiltonian (3.17) satisfies

$$
\frac{1}{\hat{H}_{P, n}^{W}-z_{n+1}}=\frac{1}{H_{P, n-1}^{W}+\left.\Delta c_{P}\right|_{n} ^{n-1}-z_{n+1}} \sum_{j=0}^{\infty}\left\{-\left.H_{I}^{W}\right|_{n} ^{n-1} \frac{1}{H_{P, n-1}^{W}+\left.\Delta c_{P}\right|_{n} ^{n-1}-z_{n+1}}\right\}^{j},
$$

for $z_{n+1} \in \gamma_{P, n+1} \cup \tilde{\gamma}_{P, n+1}$, where the equality is understood on $\mathcal{F}_{n}$ and

$$
\begin{aligned}
\left.H_{I}^{W}\right|_{n} ^{n-1} & :=\frac{1}{2}\left(\Gamma_{P, n-1} \cdot\left(\left.\mathcal{L}\right|_{n} ^{n-1}+\left.\mathcal{I}\right|_{n} ^{n-1}\right)+\text { h.c. }\right)+\frac{1}{2}\left(\left.\mathcal{L}\right|_{n} ^{n-1}+\left.\mathcal{I}\right|_{n} ^{n-1}\right)^{2}, \\
\left.\mathcal{L}\right|_{n} ^{n-1} & :=-\lambda \int_{\sigma_{n}}^{\sigma_{n-1}} d^{3} k \frac{k\left(b(k)+b^{*}(k)\right)}{\sqrt{2}|k|^{3 / 2} \alpha_{P, n-1}(\hat{k})}, \\
\left.\mathcal{I}\right|_{n} ^{n-1} & :=\lambda^{2} \bar{\int}_{\sigma_{n}}^{\sigma_{n-1}} d^{3} k \frac{k}{2|k|^{3}\left[\alpha_{P, n-1}(\hat{k})\right]^{2}}, \\
\left.\Delta c_{P}\right|_{n} ^{n-1} & :=-\lambda^{2} \int_{\sigma_{n}}^{\sigma_{n-1}} \frac{d^{3} k}{2|k|^{2} \alpha_{P, n}(\hat{k})} .
\end{aligned}
$$

Remark 3.8. We warn the reader that $\left.H_{I}^{W}\right|_{n} ^{n-1}$ depends on $P$, although this is not reflected by our notation.

Proof. By a standard computation we obtain $\hat{H}_{P, n}^{W}=H_{P, n-1}^{W}+\left.\Delta c_{P}\right|_{n} ^{n-1}+\left.H_{I}^{W}\right|_{n} ^{n-1}$. The convergence of the series follows from estimate (3.51) below. Also the restriction $|\lambda| \leq \epsilon^{4}$ enters via this estimate.

The following two lemmas are obtained again by standard computations. We remark that formula (3.31) below enters into the proof of estimate (3.19) in Lemma D.6. 
Lemma 3.9. [29] We recall that $\Pi_{P, n}$ is given by (3.22) and define

$$
\hat{\Pi}_{P, n}:=\widetilde{W}_{P, n} W_{P, n}^{*} \Pi_{P, n} W_{P, n} \widetilde{W}_{P, n}^{*} .
$$

There holds

$$
\hat{\Pi}_{P, n}-\Pi_{P, n-1}=\left.\mathcal{L}\right|_{n} ^{n-1}+\lambda^{2} \int_{\sigma_{n}}^{\sigma_{n-1}} d^{3} k \frac{k\left(\alpha_{P, n}^{2}(\hat{k})-\alpha_{P, n-1}^{2}(\hat{k})\right)}{2|k|^{3} \alpha_{P, n-1}^{2}(\hat{k}) \alpha_{P, n}^{2}(\hat{k})} .
$$

Lemma 3.10. [29] We recall that $\Gamma_{P, n}$ is given by (3.21). We define

$$
\begin{aligned}
\hat{\Gamma}_{P, n} & :=\widetilde{W}_{P, n} W_{P, n}^{*} \Gamma_{P, n} W_{P, n} \widetilde{W}_{P, n}^{*}, \\
\left.\Delta \Gamma_{P}\right|_{n} ^{n-1} & :=\hat{\Gamma}_{P, n}-\Gamma_{P, n-1}, \\
\left.\Delta^{\prime} \Gamma_{P}\right|_{n} ^{n-1} & :=-\nabla E_{P, n-1}+\nabla E_{P, n}+\left.\mathcal{L}\right|_{n} ^{n-1} .
\end{aligned}
$$

There holds

$$
\left.\Delta \Gamma_{P}\right|_{n} ^{n-1}=\left.\Delta^{\prime} \Gamma_{P}\right|_{n} ^{n-1}+\left.\mathcal{I}\right|_{n} ^{n-1}
$$

or, in other words,

$$
\hat{\Gamma}_{P, n}-\Gamma_{P, n-1}=-\nabla E_{P, n-1}+\nabla E_{P, n}+\left.\mathcal{L}\right|_{n} ^{n-1}+\left.\mathcal{I}\right|_{n} ^{n-1} .
$$

The reader should note that the difference $\hat{\Gamma}_{P, n}-\Gamma_{P, n-1}$ in formula (3.36) is controlled by the difference $-\nabla E_{P, n-1}+\nabla E_{P, n}$, which in turn depends on $\hat{\phi}_{P, n}-\phi_{P, n-1}$ via estimate (3.19). These relations will be used in the proof of Theorems 4.1 and 4.3.

In the last lemma of this subsection we analyse the ground state projections. This information will be used in the first step of the proof of Theorem 4.3.

Lemma 3.11. Recall from Remark A.11 that the ground state eigenvector of $H_{P, n}$ (defined on $\left.\mathcal{F}\right)$ is $\psi_{P, n}^{(\infty)}:=$ $\psi_{P, n} \otimes \Omega$ in $\mathcal{F}=\left.\mathcal{F}_{n} \otimes \mathcal{F}\right|_{\infty} ^{n}$. Consistently with (3.13), we define the following projections on $\mathcal{F}$ for $n \geq 1$

$$
\begin{array}{lll}
\bar{Q}_{P, n}:=\oint_{\gamma_{P, n+1}} \frac{d w_{n+1}}{H_{P, n}-w_{n+1}}, & \bar{Q}_{P, n}^{\perp}:=1-\bar{Q}_{P, n}, \\
Q_{P, n}:=W_{P, n} \bar{Q}_{P, n} W_{P, n}^{*}, & Q_{P, n}^{\perp}:=1-Q_{P, n}, \\
\hat{Q}_{P, n}:=\widetilde{W}_{P, n} W_{P, n}^{*} Q_{P, n} W_{P, n} \widetilde{W}_{P, n}^{*}, & \hat{Q}_{P, n}^{\perp}:=1-\hat{Q}_{P, n} .
\end{array}
$$

$\bar{Q}_{P, n}, Q_{P, n}, \hat{Q}_{P, n}$ leave $\mathcal{F}_{m}$ invariant for $m \geq n$. Furthermore, for $|P| \leq P_{\max }=1 / 3,|\lambda| \in\left(0, \lambda_{0}\right], \epsilon \in(0,1 / 2]$ and $|\lambda| \leq \epsilon^{4}$ there holds the following equality on $\mathcal{F}_{n}$

$$
\hat{Q}_{P, n}^{\perp}=Q_{P, n-1}^{\perp}-\oint_{\gamma_{P, n+1}} d w_{n+1} \frac{1}{H_{P, n-1}^{W}+\left.\Delta c_{P}\right|_{n} ^{n-1}-w_{n+1}} \sum_{j=1}^{\infty}\left\{-\left.H_{I}^{W}\right|_{n} ^{n-1} \frac{1}{H_{P, n-1}^{W}+\left.\Delta c_{P}\right|_{n} ^{n-1}-w_{n+1}}\right\}^{j} .
$$

Proof. Making use of Lemma 3.7, we write on $\mathcal{F}_{n}$

$$
\begin{aligned}
\hat{Q}_{P, n}^{\perp} & :=\widetilde{W}_{P, n} W_{P, n}^{*} Q_{P, n}^{\perp} W_{P, n} \widetilde{W}_{P, n}^{*} \\
& =1-\oint_{\gamma_{P, n+1}} \frac{d w_{n+1}}{\hat{H}_{P, n}^{W}-w_{n+1}} \\
& =1-\oint_{\gamma_{P, n+1}} d w_{n+1} \frac{1}{H_{P, n-1}^{W}+\left.\Delta c_{P}\right|_{n} ^{n-1}-w_{n+1}} \sum_{j=0}^{\infty}\left\{-\left.H_{I}^{W}\right|_{n} ^{n-1} \frac{1}{H_{P, n-1}^{W}+\left.\Delta c_{P}\right|_{n} ^{n-1}-w_{n+1}}\right\}^{j} .
\end{aligned}
$$


We conclude the proof by noting that on $\mathcal{F}_{n}$

$$
1-\oint_{\gamma_{P, n+1}} d w_{n+1} \frac{1}{H_{P, n-1}^{W}+\left.\Delta c_{P}\right|_{n} ^{n-1}-w_{n+1}}=1-\frac{\left(\left|\phi_{P, n-1}\right\rangle \otimes|\Omega\rangle\right)\left(\left\langle\phi_{P, n-1}\right| \otimes\langle\Omega|\right)}{\left\|\phi_{P, n-1} \otimes \Omega\right\|^{2}}=Q_{P, n-1}^{\perp} .
$$

Here we need that the resolvent is well defined and the contour $-\left.\Delta c_{P}\right|_{n} ^{n-1}+\gamma_{P, n+1}$ encloses no spectral point of $H_{P, n-1}^{W}$ except for the ground state energy. Since (cf. Proposition 3.2 and formula (3.29)) $\left|\Delta c_{P}\right|_{n}^{n-1} \mid \leq c \lambda^{2} \sigma_{n-1}$, $\left|E_{P, n-1}-E_{P, n}\right| \leq c_{\Delta E} \lambda^{2} \sigma_{n-1},\left|w_{n+1}-E_{P, n}\right|=\frac{\sigma_{n+1}}{3}$, and $\operatorname{Gap}\left(H_{P, n-1} 1 \mathcal{F}_{n}\right) \geq \frac{\sigma_{n}}{2}$, this can be achieved, uniformly in $n$, by choosing $\lambda_{0}$ sufficiently small. Now the first equality in (3.42) follows from Proposition 3.2.

\subsection{Standard resolvent bounds}

In this subsection we collect some standard bounds on the quantities introduced above which will be heavily used in the proofs of Theorems 4.1 and 4.3. The proof of the following lemma is given in Appendix B.

Lemma 3.12. Define

$$
\begin{aligned}
H_{P, n-1}^{W+} & :=H_{P, n-1}^{W}+\left.\Delta c_{P}\right|_{n} ^{n-1} \\
\left(\left.H_{I}^{W}\right|_{n} ^{n-1}\right)_{\text {mix }} & :=\frac{1}{2}\left(\Gamma_{P, n-1} \cdot\left(\left.\mathcal{L}\right|_{n} ^{n-1}+\left.\mathcal{I}\right|_{n} ^{n-1}\right)+\text { h.c. }\right), \\
\Delta\left(\left.H_{I}^{W}\right|_{n} ^{n-1}\right)_{\text {mix }} & :=\left(\left.H_{I}^{W}\right|_{n} ^{n-1}\right)_{\text {mix }}-\left(\left.\mathcal{L}\right|_{n} ^{n-1}+\left.\mathcal{I}\right|_{n} ^{n-1}\right) \cdot \Gamma_{P, n-1} \\
& =\sum_{i=1}^{3} \frac{1}{2}\left[\left(\Gamma_{P, n-1}\right)_{i},\left(\left.\mathcal{L}\right|_{n} ^{n-1}\right)_{i}\right] \\
\left(\left.H_{I}^{W}\right|_{n} ^{n-1}\right)_{\text {quad }} & :=\left.H_{I}^{W}\right|_{n} ^{n-1}-\left(\left.H_{I}^{W}\right|_{n} ^{n-1}\right)_{\text {mix }}=\frac{1}{2}\left(\left.\mathcal{L}\right|_{n} ^{n-1}+\left.\mathcal{I}\right|_{n} ^{n-1}\right)^{2} .
\end{aligned}
$$

Let $|P| \leq P_{\max }=1 / 3,|\lambda| \in\left(0, \lambda_{0}\right], \epsilon \in(0,1 / 2]$ and $|\lambda| \leq \epsilon^{4}$. Then, for $z_{n+1} \in \tilde{\gamma}_{P, n+1} \cup \gamma_{P, n+1}$, the following estimates hold true for universal constants $c_{0}, c_{2}, c_{3}, c_{4}$ :

$$
\begin{aligned}
& \left.\left\|\frac{1}{H_{P, n-1}^{W+}-z_{n+1}}\right\|\right|_{\mathcal{F}_{n}} \leq \frac{c_{0}}{\sigma_{n+1}}, \\
& \left\|\frac{1}{H_{P, n-1}^{W+}-z_{n+1}}\left(\Gamma_{P, n-1}\right)_{i}\right\|_{\mathcal{F}_{n}} \leq \frac{c_{0}}{\sigma_{n+1}} \\
& \left\|\frac{1}{H_{P, n-1}^{W+}-z_{n+1}}\left(\left.\mathcal{I}\right|_{n} ^{n-1}\right)_{i}\right\|_{\mathcal{F}_{n}} \leq c_{2}|\lambda|^{\frac{1}{2}}, \\
& \left\|\frac{1}{H_{P, n-1}^{W+}-z_{n+1}}\left(\left.\mathcal{L}\right|_{n} ^{n-1}\right)_{i}\right\|_{\mathcal{F}_{n}} \leq c_{2}|\lambda|^{\frac{1}{2}}, \\
& \left\|\frac{1}{H_{P, n-1}^{W+}-z_{n+1}}\left(\left.H_{I}^{W}\right|_{n} ^{n-1}\right)\right\|_{\mathcal{F}_{n}} \leq c_{2}|\lambda|^{\frac{1}{2}}, \\
& \left\|\frac{1}{H_{P, n-1}^{W+}-z_{n+1}}\left(\left.H_{I}^{W}\right|_{n} ^{n-1}\right)_{\text {quad }}\right\|_{\mathcal{F}_{n}} \leq c_{3}|\lambda| \sigma_{n-1}, \\
& \left\|\frac{1}{H_{P, n-1}^{W+}-z_{n+1}} \Delta\left(\left.H_{I}^{W}\right|_{n} ^{n-1}\right)_{\text {mix }}\right\|_{\mathcal{F}_{n}} \leq c_{4}|\lambda|^{\frac{1}{2}} \sigma_{n-1} .
\end{aligned}
$$

All estimates are uniform in $z_{n+1}$ within the specified restrictions. The estimate in (3.50) still holds after replacing $\left(\left.\mathcal{L}\right|_{n} ^{n-1}\right)_{i}$ with its creation or annihilation parts $\left(\left.\mathcal{L}\right|_{n} ^{n-1}\right)_{i}^{( \pm)}$. The estimate in (3.49) can readily be improved to $c_{2}|\lambda|^{3 / 2}(c f .(B .11))$, but it will be convenient to have the same bound in (3.49)-(3.51). 
Remark 3.13. It will be convenient to assume in the following that

$$
\begin{aligned}
\left|E_{P, n-1}-E_{P, n}\right| & \leq \frac{1}{20} \sigma_{n+1}, \\
\left|\Delta c_{P}\right|_{n}^{n-1} \mid & \leq \frac{1}{20} \sigma_{n+1} .
\end{aligned}
$$

By (3.29) and (3.10) this can be ensured by reducing $\lambda_{0}$ and using $|\lambda| \leq \epsilon^{4}$. Furthermore, we require that

$$
c_{2}\left|\lambda_{0}\right|^{1 / 2} \leq 1 / 2, \quad c_{0} c_{2}\left|\lambda_{0}\right|^{1 / 4} \leq 1 / 2,
$$

where $c_{0}, c_{2}$ are universal constants appearing in Lemma 3.12. This is needed, in particular, in (C.3) below.

\subsection{Direct integral representations}

In this subsection we introduce direct integral representations of transformed Hamiltonians which simplify the proof of Theorem 4.1 and are crucial for the proof of Theorem 4.3. The relevance of such representations for iterative analytic perturbation theory was noticed in [27] (see e.g. Step 1 of the proof of Lemma 6.1 of this reference).

Let us first introduce the following auxiliary Hamiltonians, acting on the Hilbert space $\mathcal{F}$, for $k_{1}, \ldots, k_{m}$ s.t. $\sigma_{n} \leq\left|k_{\ell}\right| \leq \sigma_{n-1}$ and $n \geq 1$ :

$$
\begin{aligned}
{\left[H_{P, n-1}^{W+}\right]_{k_{1}, \ldots, k_{m}}: } & =H_{P, n-1}^{W+}+\sum_{\ell=1}^{m}\left(k_{\ell} \cdot \Gamma_{P, n-1}+\alpha_{P, n-1}\left(\hat{k}_{\ell}\right)\left|k_{\ell}\right|\right)+\frac{\left(\sum_{\ell=1}^{m} k_{\ell}\right)^{2}}{2} \\
& =W_{P, n-1} H_{P-\sum_{\ell=1}^{m} k_{\ell}, n-1} W_{P, n-1}^{*}+\left.\Delta c_{P}\right|_{n} ^{n-1}+\sum_{\ell=1}^{m}\left|k_{\ell}\right|,
\end{aligned}
$$

where the step from (3.57) to (3.58) uses (3.16), (3.21), and (3.43).

To exhibit the geometric meaning of these Hamiltonians we recall that $\mathcal{F}_{n}=\left.\mathcal{F}_{n-1} \otimes \mathcal{F}\right|_{n} ^{n-1}$ and $\left.\mathcal{F}\right|_{n} ^{n-1}$ is the direct sum of $m$-particle subspaces $\left(\left.\mathcal{F}\right|_{n} ^{n-1}\right)^{(m)}, m \geq 0$. We have $\left(\left.\mathcal{F}\right|_{n} ^{n-1}\right)^{(1)}:=L^{2}\left(\left.\mathcal{A}\right|_{n} ^{n-1}, d^{3} k\right)$ and $\left(\left.\mathcal{F}\right|_{n} ^{n-1}\right)^{(m)}:=L_{\mathrm{S}}^{2}\left(\left(\left.\mathcal{A}\right|_{n} ^{n-1}\right)^{\times m}, d^{3 m} k\right)$, where the subscript s denotes symmetric subspace. Thus we have the standard identifications

$$
\begin{aligned}
& \mathcal{F}_{n-1} \otimes\left(\left.\mathcal{F}\right|_{n} ^{n-1}\right)^{(1)} \simeq \int_{\left.\mathcal{A}\right|_{n} ^{n-1}}^{\oplus} d^{3} k \mathcal{F}_{n-1}, \\
& \mathcal{F}_{n-1} \otimes\left(\left.\mathcal{F}\right|_{n} ^{n-1}\right)^{(m)} \simeq\left[\int_{\left(\left.\mathcal{A}\right|_{n} ^{n-1}\right)^{\times m}}^{\oplus} d^{3 m} k \mathcal{F}_{n-1}\right]_{\mathrm{s}},
\end{aligned}
$$

where $[\ldots]_{\mathrm{S}}$ denotes the subspace of symmetric sections. In these terms we can write (e.g. in the sense of functional calculus)

$$
\begin{aligned}
H_{P, n-1}^{W+} 1\left(\mathcal{F}_{n-1} \otimes\left(\left.\mathcal{F}\right|_{n} ^{n-1}\right)^{(m)}\right) & \simeq \int_{\left(\left.\mathcal{A}\right|_{n} ^{n-1}\right)^{\times m}}^{\oplus} d^{3 m} k\left(\left[H_{P, n-1}^{W+}\right]_{k_{1}, \ldots, k_{m}} 1 \mathcal{F}_{n-1}\right), \\
\Gamma_{P, n-1} 1\left(\mathcal{F}_{n-1} \otimes\left(\left.\mathcal{F}\right|_{n} ^{n-1}\right)^{(m)}\right) & \simeq \int_{\left(\left.\mathcal{A}\right|_{n} ^{n-1}\right)^{\times m}}^{\oplus} d^{3 m} k\left(\left(\Gamma_{P, n-1}+\sum_{\ell=1}^{m} k_{\ell}\right) 1 \mathcal{F}_{n-1}\right) .
\end{aligned}
$$

To justify (3.61) for $m=1$ it suffices to write

$$
\begin{aligned}
& H_{P, n-1}^{W+} 1\left(\mathcal{F}_{n-1} \otimes\left(\left.\mathcal{F}\right|_{n} ^{n-1}\right)^{(1)}\right) \\
& =W_{P, n-1}\left(\frac{1}{2}\left(P-\left.P_{\mathrm{f}}\right|_{n-1} ^{0} \otimes 1-\left.1 \otimes k\right|_{n} ^{n-1}\right)^{2}+\left.H_{\mathrm{f}}\right|_{n-1} ^{0} \otimes 1+\left.1 \otimes|k|\right|_{n} ^{n-1}+\left.\lambda \Phi\right|_{n-1} ^{0} \otimes 1+\left.1 \otimes \Delta c_{P}\right|_{n} ^{n-1}\right) W_{P, n-1}^{*},
\end{aligned}
$$


where $\left.k\right|_{n} ^{n-1},\left.|k|\right|_{n} ^{n-1}$ are multiplication operators on $L^{2}\left(\left.\mathcal{A}\right|_{n} ^{n-1}, d^{3} k\right)$ and $\left.\Phi\right|_{n-1} ^{0},\left.H_{\mathrm{f}}\right|_{n-1} ^{0},\left.P_{\mathrm{f}}\right|_{n-1} ^{0}$ are defined in (A.1)(A.3). The case $m>1$ is treated analogously.

Example. To illustrate the above definitions we write the direct integral representation of the following expression which appears in Lemma 4.16 below:

$$
\begin{aligned}
& \frac{1}{H_{P, n-1}^{W+}-z_{n+1}}\left(\Gamma_{P, n-1}\right)_{i} \frac{1}{H_{P, n-1}^{W+}-z_{n+1}^{\prime}}\left(\left.\mathcal{L}\right|_{n} ^{n-1}\right)_{i^{\prime}}^{(+)} \phi_{P, n-1} \\
& \simeq-\lambda \int_{\left.\mathcal{P}\right|_{n} ^{n-1}}^{\oplus} \frac{d^{3} k}{\sqrt{2}|k|^{3 / 2}} \frac{k_{i^{\prime}}}{\alpha_{P, n-1}(\hat{k})} \frac{1}{\left[H_{P, n-1}^{W+}\right]_{k}-z_{n+1}}\left(\Gamma_{P, n-1}+k\right)_{i} \frac{1}{\left[H_{P, n-1}^{W+}\right]_{k}-z_{n+1}^{\prime}} \phi_{P, n-1},
\end{aligned}
$$

where $z_{n+1}, z_{n+1}^{\prime} \in \tilde{\gamma}_{P, n+1}$ and we used definition (3.27):

$$
\left(\left.\mathcal{L}\right|_{n} ^{n-1}\right)_{i^{\prime}}^{(+)}:=-\lambda \int_{\left.\mathcal{A}\right|_{n} ^{n-1}} \frac{d^{3} k}{\sqrt{2}|k|^{3 / 2}} \frac{k_{i^{\prime}}}{\alpha_{P, n-1}(\hat{k})} b^{*}(k) .
$$

By standard properties of direct integrals, we also have

$$
\|(3.64)\|=|\lambda|\left(\int_{\left.\mathcal{P}\right|_{n} ^{n-1}} \frac{d^{3} k}{2|k|^{3}} \frac{k_{i^{\prime}}^{2}}{\alpha_{P, n-1}(\hat{k})^{2}}\left\|\frac{1}{\left[H_{P, n-1}^{W+}\right]_{k}-z_{n+1}}\left(\Gamma_{P, n-1}+k\right)_{i} \frac{1}{\left[H_{P, n-1}^{W+}\right]_{k}-z_{n+1}^{\prime}} \phi_{P, n-1}\right\|^{2}\right)^{1 / 2} .
$$

There is a different way to do the above computations, which we only sketch, as it will not be used in the following. Considering that for $\sigma_{n} \leq|k| \leq \sigma_{n-1}$

$$
\frac{1}{H_{P, n-1}^{W+}-z_{n+1}} b^{*}(k)=b^{*}(k) \frac{1}{\left[H_{P, n-1}^{W+}\right]_{k}-z_{n+1}}, \quad \Gamma_{P, n-1} b^{*}(k)=b^{*}(k)\left(\Gamma_{P, n-1}+k\right),
$$

and referring to definition (3.66), we can simply commute $\left(\left.\mathcal{L}\right|_{n} ^{n-1}\right)_{i^{\prime}}^{(+)}$to the left in (3.64), obtaining

$$
\text { (3.64) }=-\lambda \int_{\left.\mathcal{A}\right|_{n} ^{n-1}} \frac{d^{3} k}{\sqrt{2}|k|^{3 / 2}} \frac{k_{i^{\prime}}}{\alpha_{P, n-1}(\hat{k})} b^{*}(k) \frac{1}{\left[H_{P, n-1}^{W+}\right]_{k}-z_{n+1}}\left(\Gamma_{P, n-1}+k\right)_{i} \frac{1}{\left[H_{P, n-1}^{W+}\right]_{k}-z_{n+1}^{\prime}} \phi_{P, n-1} .
$$

This gives (3.67) by an obvious computation using $\left[b(k), b^{*}\left(k^{\prime}\right)\right]=\delta\left(k-k^{\prime}\right)$ and $b(k) \phi_{P, n-1}=0$ for $\sigma_{n} \leq|k| \leq$ $\sigma_{n-1}$. We remark that (3.65) can be seen as a rigorous implementation of (3.69).

In the following lemma we collect the relevant properties of $\left[H_{P, n-1}^{W+}\right]_{k_{1}, \ldots, k_{m}}$. We recall that they are understood as operators on $\mathcal{F}$.

Lemma 3.14. Suppose that $|P| \leq P_{\max }=1 / 3,|\lambda| \in\left(0, \lambda_{0}\right], \epsilon \in(0,1 / 2]$ and $|\lambda| \leq \epsilon^{4}$. Then, for $\sigma_{n} \leq$ $\left|k_{1}\right|, \ldots,\left|k_{m}\right| \leq \sigma_{n-1}, n \in \mathbb{N}$ and $z_{n+1} \in \gamma_{P, n+1} \cup \tilde{\gamma}_{P, n+1}$

$$
\begin{aligned}
& \left\|\frac{1}{\left[H_{P, n-1}^{W+}\right]_{k_{1}, \ldots, k_{m}}-z_{n+1}}\right\| \leq \frac{c}{\left|k_{1}\right|+\cdots+\left|k_{m}\right|}, \\
& \left\|\frac{1}{\left[H_{P, n-1}^{W+}\right]_{k_{1}, \ldots, k_{m}}-z_{n+1}}\left(\Gamma_{P, n-1}\right)_{i}\right\| \leq \frac{c m}{\left|k_{1}\right|+\cdots+\left|k_{m}\right|} .
\end{aligned}
$$

Furthermore, for any $\phi \in \mathcal{F}_{n}$

$$
\left\|\frac{1}{\left[H_{P, n-1}^{W+}\right]_{k_{1}, \ldots, k_{m}}-z_{n+1}} Q_{P, n-1}^{\perp}\left(\Gamma_{P, n-1}\right)_{i} \phi\right\| \leq c m\left\|Q_{P, n-1}^{\perp} \frac{1}{H_{P, n-1}^{W+}-z_{n+1}}\left(\Gamma_{P, n-1}\right)_{i} \phi\right\|,
$$

where the r.h.s. is well defined by (3.48). 
Proof. We treat only the case of $z_{n+1} \in \tilde{\gamma}_{P, n+1}$ as the case of circle contours is analogous and simpler. Making use of representation (3.58), we estimate

$$
\begin{aligned}
{\left[H_{P, n-1}^{W+}\right]_{k_{1}, \ldots, k_{m}}-\operatorname{Re} z_{n+1}=} & W_{P, n-1}\left(H_{P-\sum_{\ell=1}^{m} k_{\ell}, n-1}-E_{P-\sum_{\ell=1}^{m} k_{\ell, n-1}}\right) W_{P, n-1}^{*} \\
& +\left(E_{P-\sum_{\ell=1}^{m} k_{\ell}, n-1}-E_{P, n-1}\right)+\left(E_{P, n-1}-E_{P, n}\right) \\
& +\left(E_{P, n}-\operatorname{Re} z_{n+1}\right)+\left.\Delta c_{P}\right|_{n} ^{n-1}+\sum_{\ell=1}^{m}\left|k_{\ell}\right| \\
\geq & -(1 / 3+c|\lambda|)\left|\sum_{\ell=1}^{m} k_{\ell}\right|-(1 / 3+1 / 10) \sigma_{n+1}+\sum_{\ell=1}^{m}\left|k_{\ell}\right| \\
\geq & -(1 / 3+c|\lambda|) \sum_{\ell=1}^{m}\left|k_{\ell}\right|+(1 / 2) \sum_{\ell=1}^{m}\left|k_{\ell}\right| \\
& +(1 / 2)\left(\sum_{\ell=1}^{m}\left|k_{\ell}\right|\right)-(1 / 2)(1 / 3+1 / 10)(1 / m)\left(\sum_{\ell=1}^{m}\left|k_{\ell}\right|\right) \geq c^{\prime}\left(\sum_{\ell=1}^{m}\left|k_{\ell}\right|\right)
\end{aligned}
$$

for $c^{\prime}>0$ and $\lambda_{0}$ sufficiently small, both independent of $m$. Here we made use of Remark A.11, which tells us that $E_{P-\sum_{\ell=1}^{m} k_{\ell}, n-1}$ is the infimum of the spectrum of $H_{P-\sum_{\ell=1}^{m} k_{\ell}, n-1}$ over the full Fock space $\mathcal{F}$. (Therefore the norms in (3.70), (3.71) can be taken over $\mathcal{F}$ ). Furthermore, in (3.73) we used Proposition 3.2, restrictions (3.54), (3.55) and $\sigma_{n+1} \leq(1 / 2) \sigma_{n} \leq(1 / 2)(1 / m) \sum_{\ell=1}^{m}\left|k_{\ell}\right|$. This gives the estimate in (3.70).

Now we consider the bound in (3.71). We recall that by assumption $\sigma_{n} \leq\left|k_{\ell}\right| \leq \sigma_{n-1} \leq 1$. Then, making use of (3.58), (3.70) and $\left|\nabla E_{P, n}\right| \leq \frac{1}{3}+c|\lambda|$ we have, setting $A\left(z_{n+1}\right):=H_{P-\sum_{\ell=1}^{m} k_{\ell, n-1}}+\left.\Delta c_{P}\right|_{n} ^{n-1}+\sum_{\ell=1}^{m}\left|k_{\ell}\right|-z_{n+1}$,

$$
\begin{aligned}
\left\|\frac{1}{\left[H_{P, n-1}^{W+}\right]_{k}-z_{n+1}}\left(\Gamma_{P, n-1}\right)_{i}\right\| & =\left\|A\left(z_{n+1}\right)^{-1}\left(-\left(P-P_{\mathrm{f}}\right)+\nabla E_{P, n}\right)_{i}\right\| \\
& \leq\left\|A\left(z_{n+1}\right)^{-1}\left(P-\sum_{\ell=1}^{m} k_{\ell}-P_{\mathrm{f}}\right)_{i}\right\|+O\left(m\left(\sum_{\ell=1}^{m}\left|k_{\ell}\right|\right)^{-1}\right) \\
& \leq c\left\|A\left(z_{n+1}\right)^{-1} H_{P-\sum_{\ell=1}^{m} k_{\ell}, \text { free }} A\left(\bar{z}_{n+1}\right)^{-1}\right\|^{1 / 2}+O\left(m\left(\sum_{\ell=1}^{m}\left|k_{\ell}\right|\right)^{-1}\right) \\
& \leq c\left\|A\left(z_{n+1}\right)^{-1} H_{P-\sum_{\ell=1}^{m} k_{\ell}, n-1} A\left(\bar{z}_{n+1}\right)^{-1}\right\|^{1 / 2}+O\left(m\left(\sum_{\ell=1}^{m}\left|k_{\ell}\right|\right)^{-1}\right),
\end{aligned}
$$

where in the last step we made use of Lemma A.3 and again of (3.70). By writing $H_{P-\sum_{\ell=1}^{m} k_{\ell}, n-1}=A\left(z_{n+1}\right)-$ $\left(\left.\Delta c_{P}\right|_{n} ^{n-1}+\sum_{\ell=1}^{m}\left|k_{\ell}\right|-z_{n+1}\right)$, considering separately the case $\left|\operatorname{Im} z_{n+1}\right| \leq 1$ and $\left|\operatorname{Im} z_{n+1}\right| \geq 1$, and making use again of (3.70) we conclude the proof of (3.71).

To show estimate (3.72), we apply the resolvent expansion truncated at first order using representation (3.57) of the Hamiltonian

$$
\begin{aligned}
\frac{1}{\left[H_{P, n-1}^{W+}\right]_{k_{1}, \ldots, k_{m}}-z_{n+1}}= & \frac{1}{H_{P, n-1}^{W+}-z_{n+1}} \\
& +\frac{1}{\left[H_{P, n-1}^{W+}\right]_{k_{1}, \ldots, k_{m}}-z_{n+1}}\left[-\sum_{\ell=1}^{m}\left(k_{\ell} \cdot \Gamma_{P, n-1}+\alpha_{P, n-1}\left(\hat{k}_{\ell}\right)\left|k_{\ell}\right|\right)-\frac{1}{2}\left(\sum_{\ell=1}^{m} k_{\ell}\right)^{2}\right] \frac{1}{H_{P, n-1}^{W+}-z_{n+1}} .
\end{aligned}
$$

Now, using the bounds (3.70), (3.71) and the constraint $\sigma_{n} \leq\left|k_{\ell}\right| \leq \sigma_{n-1} \leq 1$, we obtain

$$
\left\|\frac{1}{\left[H_{P, n-1}^{W+}\right]_{k_{1}, \ldots, k_{m}}-z_{n+1}} Q_{P, n-1}^{\perp}\left(\Gamma_{P, n-1}\right)_{i} \phi\right\| \leq c m\left\|Q_{P, n-1}^{\perp} \frac{1}{H_{P, n-1}^{W+}-z_{n+1}}\left(\Gamma_{P, n-1}\right)_{i} \phi\right\| .
$$


This concludes the proof of (3.72) and of the lemma.

\subsection{Maximal modulus principle arguments}

We recall the standard maximal modulus principle:

Theorem 3.15. Let $f$ be a holomorphic function of $m$ complex variables with a bounded connected region of holomorphy $O \subset \mathbb{C}^{m}$, which extends by continuity to the boundary $\partial O$. Then

$$
\sup _{z \in O}|f(z)|=\sup _{z \in \partial O}|f(z)| .
$$

Now we state a simple application:

Lemma 3.16. Let $F$ be a Hilbert space valued function of $m$ complex variables, holomorphic in

$$
\tilde{\gamma}_{P, n}^{-}:=\left\{\underline{z}:=\left(z_{1}, \ldots, z_{m}\right) \in \mathbb{C}^{m} \mid \operatorname{Re} z_{i}<E_{P, n-1}+\sigma_{n} / 3, i=1, \ldots, m\right\},
$$

which extends by continuity to the closure of this region. Suppose furthermore that

$$
\lim _{R \rightarrow \infty} \sup _{\underline{z} \in C_{R, n}^{\times m}}\|F(\underline{z})\|=0
$$

where $C_{R, n}:=\left\{z \in \mathbb{C} \mid z=E_{P, n-1}+\sigma_{n} / 3+R e^{i \phi}, \phi \in[\pi / 2,3 \pi / 2]\right\}$. Then, for any $\underline{z} \in \tilde{\gamma}_{P, n}^{-}$,

$$
\|F(\underline{z})\| \leq \sup _{\underline{z^{\prime}} \in \tilde{\gamma}_{P, n}^{\times m}}\left\|F\left(\underline{z^{\prime}}\right)\right\|
$$

where $\tilde{\gamma}_{P, n}$ is defined in (3.24).

Proof. For any $\psi \in \mathcal{H}$ with $\|\psi\|=1$ consider $f_{\psi}(\underline{z}):=\langle\psi, F(\underline{z})\rangle$. We note that we cannot apply Theorem 3.15 directly to $f_{\psi}$, because the region $\tilde{\gamma}_{P, n}^{-}$is unbounded. Let $O_{R, n} \subset \mathbb{C}$ be the bounded region limited by the line $\tilde{\gamma}_{P, n}$ and the half-circle $C_{R, n}$. Given $\underline{z} \in \tilde{\gamma}_{P, n}^{-}$we choose $R$ large enough so that $\underline{z} \in O_{R, n}^{\times m}$. Then, by (3.77)

$$
\left|f_{\psi}(\underline{z})\right| \leq \sup _{\underline{z}^{\prime} \in \partial O_{R, n}^{\times m}}\left|f_{\psi}\left(\underline{z}^{\prime}\right)\right| \leq \max \left\{\sup _{\underline{z}^{\prime} \in \tilde{\gamma}_{P, n}^{\times n}}\left|f_{\psi}\left(\underline{z^{\prime}}\right)\right|, \sup _{\underline{z}^{\prime} \in C_{R, n}^{\times m}}\left|f_{\psi}\left(\underline{z}^{\prime}\right)\right|\right\}=\sup _{\underline{z}^{\prime} \in \tilde{\gamma}_{P, n}^{\times m}}\left|f_{\psi}\left(\underline{z}^{\prime}\right)\right| \leq \sup _{\underline{z}^{\prime} \in \tilde{\gamma}_{P, n}^{\times m}}\left\|F\left(\underline{z}^{\prime}\right)\right\|,
$$

where in the third step we took $R$ sufficiently large and used (3.79). Now (3.80) follows by taking the supremum over $\psi$.

\section{Main technical result}

In this section we state and prove our main technical result, which is Theorem 4.3 below. To our knowledge this result goes beyond the existing applications of iterative analytic perturbation theory. The proof relies on Theorem 4.1 stated below, which is proven in Appendix C. We recall that results comparable to Theorem 4.1 were obtained in $[23,27]$ for different models but the case of the Nelson model was left aside.

Theorem 4.1. Let $|P| \leq P_{\max }=1 / 3$. Then, for any $\delta$, with $\frac{1}{4}>\delta>0$, there exist $\epsilon(\delta)>0$ and $\lambda_{0} \equiv \lambda_{0}(\epsilon(\delta))>0$ with the following property: For any $\epsilon \in(0, \epsilon(\delta)]$ and $|\lambda| \in\left(0, \lambda_{0}\right]$ s.t. $|\lambda| \leq \epsilon^{8}$ we have for $n \geq 0$ :

i)

$$
\sup _{z_{n+1} \in \tilde{\gamma} P, n+1}\left\|\frac{1}{H_{P, n}^{W}-z_{n+1}}\left(\Gamma_{P, n}\right)_{i} \phi_{P, n}\right\| \leq \frac{1}{\sigma_{n}^{\delta}}, \quad i=1,2,3,
$$


ii)

$$
\left\|\hat{\phi}_{P, n+1}-\phi_{P, n}\right\| \leq|\lambda|^{\frac{1}{4}} \sigma_{n}^{1-\delta},
$$

iii)

$$
\left\|\phi_{P, n+1}\right\| \geq 1-2 \sum_{j=0}^{n}\left\{|\lambda|^{\frac{1}{4}} \sigma_{j}^{\frac{1}{2}}\right\} \geq \frac{1}{2} .
$$

Remark 4.2. Making use of the auxiliary bounds (3.54), (3.55), it is easy to see that for $z_{n+1} \in \tilde{\gamma}_{P, n+1}, z_{n+2} \in$ $\tilde{\gamma}_{P, n+2}$ we have $\operatorname{Re} z_{n+2}-\left.\Delta c_{P}\right|_{n+1} ^{n} \leq \operatorname{Re} z_{n+1}$. Given this, it is an immediate consequence of claim i) of Theorem 4.1 and the maximal modulus principle (Lemma 3.16) that the first estimate below holds

$$
\sup _{z_{n+2} \in \tilde{\gamma}_{P, n+2}}\left\|\frac{1}{H_{P, n}^{W+}-z_{n+2}}\left(\Gamma_{P, n}\right)_{i} \phi_{P, n}\right\| \leq \frac{1}{\sigma_{n}^{\delta}}, \quad \sup _{z_{n+1} \in \tilde{\gamma}_{P, n+1}}\left\|\frac{1}{H_{P, n-1}^{W+}-z_{n+1}}\left(\Gamma_{P, n-1}\right)_{i} \phi_{P, n-1}\right\| \leq \frac{1}{\sigma_{n-1}^{\delta}} .
$$

Here the gap estimate (3.11) and the fact that $\Gamma_{P, n} \phi_{P, n}$ is orthogonal to $\phi_{P, n}$ ensured the holomorphy in the relevant region. The second inequality above follows analogously (for $n \geq 1$ ) from claim i) for $n \rightarrow n-1$ and thus it can be used in the inductive proof of Theorem 4.1. (It is also useful in the proof of Theorem 4.3 below). Alluding again to the maximal modulus principle, we can replace $\tilde{\gamma}_{P, n+2}, \tilde{\gamma}_{P, n+1}$ with $\gamma_{P, n+2}, \gamma_{P, n+1}$ in (4.4).

It may be demanding for the reader to scrutinize the proof of the following theorem. We suggest that he/she opens two copies of the paper on a large computer screen to have both the main line of the argument and the relevant auxiliary lemmas and definitions simultaneously in front of his/her eyes.

Theorem 4.3. Let $|P| \leq P_{\max }=1 / 3$. Then, for any $\delta$, with $\frac{1}{4}>\delta>0$, there exist $\epsilon^{*}(\delta)>0$ and $\lambda_{0}^{*} \equiv \lambda_{0}^{*}\left(\epsilon^{*}(\delta)\right)>$ 0 with the following property: For any $\epsilon \in\left(0, \epsilon^{*}(\delta)\right]$ and $|\lambda| \in\left(0, \lambda_{0}^{*}\right]$ s.t. $|\lambda| \leq \epsilon^{8}$ we have for $n \geq 0$ :

$$
\sup _{z_{n+1}, z_{n+1}^{\prime} \in \tilde{\gamma}_{P, n+1}}\left\|\frac{Q_{P, n}^{\perp}}{H_{P, n}^{W}-z_{n+1}}\left(\Gamma_{P, n}\right)_{i} \frac{1}{H_{P, n}^{W}-z_{n+1}^{\prime}}\left(\Gamma_{P, n}\right)_{i^{\prime}} \phi_{P, n}\right\| \leq \frac{1}{\sigma_{n}^{2 \delta}} .
$$

Remark 4.4. Obviously, we have $\lambda_{0}^{*} \in\left(0, \lambda_{0}\right]$ and $\epsilon^{*}(\delta) \in(0, \epsilon(\delta)]$, where $\lambda_{0}$ and $\epsilon(\delta)$ are the values fixed in Theorem 4.1.

The remaining part of this section is devoted to the proof of Theorem 4.3 which is divided into several subsections. The inequality in (4.5) is manifestly true for $n=0$ because $\left(\Gamma_{P, 0}\right)_{i} \phi_{P, 0}=0$. Therefore we can assume that (4.5) is fulfilled for $n-1(n \geq 1)$ and prove that, consequently, it holds for $n$. Our induction hypothesis has the form

$$
\sup _{z_{n}, z_{n}^{\prime} \in \tilde{\gamma}_{P, n}}\left\|\frac{Q_{P, n-1}^{\perp}}{H_{P, n-1}^{W}-z_{n}}\left(\Gamma_{P, n-1}\right)_{i} \frac{1}{H_{P, n-1}^{W}-z_{n}^{\prime}}\left(\Gamma_{P, n-1}\right)_{i^{\prime}} \phi_{P, n-1}\right\| \leq \frac{1}{\sigma_{n-1}^{2 \delta}} .
$$

Remark 4.5. Similarly as in (4.4), with the help of the maximal modulus principle (Lemma 3.16), inequality (4.6) implies an estimate which is more convenient in applications:

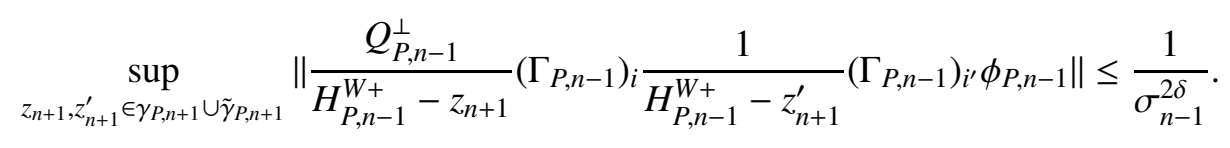

We also remark that in the following discussion $z_{n+1}, z_{n+1}^{\prime}$ belong to $\tilde{\gamma}_{P, n+1}$ (or to $\gamma_{P, n+1} \cup \tilde{\gamma}_{P, n+1}$ if explicitly specified), whereas $w_{n+1}, w_{n+1}^{\prime}$ belong to $\gamma_{P, n+1}$. 
Proceeding to the inductive proof, we use the unitary operator $\widetilde{W}_{P, n} W_{P, n}^{*}$ to switch in (4.5) from the given expression to the corresponding expression with 'hats'

$$
\left\|\frac{Q_{P, n}^{\perp}}{H_{P, n}^{W}-z_{n+1}}\left(\Gamma_{P, n}\right)_{i} \frac{1}{H_{P, n}^{W}-z_{n+1}^{\prime}}\left(\Gamma_{P, n}\right)_{i^{\prime}} \phi_{P, n}\right\|=\left\|\frac{\hat{Q}_{P, n}^{\perp}}{\hat{H}_{P, n}^{W}-z_{n+1}}\left(\hat{\Gamma}_{P, n}\right)_{i} \frac{1}{\hat{H}_{P, n}^{W}-z_{n+1}^{\prime}}\left(\hat{\Gamma}_{P, n}\right)_{i^{\prime}} \hat{\phi}_{P, n}\right\|,
$$

where $z_{n+1}, z_{n+1}^{\prime} \in \tilde{\gamma}_{P, n+1}$. Using the definition in (3.43) and formulae (3.25), (3.33), and (3.40), we proceed with the full expansion of $\hat{Q}_{P, n}^{\perp}, \hat{\phi}_{P, n}, \frac{1}{\hat{H}_{P, n}^{W}-z_{n+1}}$, and $\left(\hat{\Gamma}_{P, n}\right)_{i}$ :

$$
\begin{aligned}
\hat{Q}_{P, n}^{\perp} & \frac{1}{\hat{H}_{P, n}^{W}-z_{n+1}}\left(\hat{\Gamma}_{P, n}\right)_{i} \frac{1}{\hat{H}_{P, n}^{W}-z_{n+1}^{\prime}}\left(\hat{\Gamma}_{P, n}\right)_{i^{\prime}} \hat{\phi}_{P, n} \\
= & \left\{Q_{P, n-1}^{\perp}-\sum_{r=1}^{\infty} \oint_{\gamma_{P, n+1}} d w_{n+1}^{\prime}\left\{\left.\frac{1}{H_{P, n-1}^{W+}-w_{n+1}^{\prime}}(-) H_{I}^{W}\right|_{n} ^{n-1}\right\}^{r} \frac{1}{H_{P, n-1}^{W+}-w_{n+1}^{\prime}}\right\} \times \\
\times & \sum_{l=0}^{\infty}\left\{\left.\frac{1}{H_{P, n-1}^{W+}-z_{n+1}}(-) H_{I}^{W}\right|_{n} ^{n-1}\right\}^{l} \frac{1}{H_{P, n-1}^{W+}-z_{n+1}} \times \\
& \times\left(\Gamma_{P, n-1}+\left.\Delta \Gamma_{P}\right|_{n} ^{n-1}\right)_{i} \times \\
& \times \sum_{l^{\prime}=0}^{\infty}\left\{\left.\frac{1}{H_{P, n-1}^{W+}-z_{n+1}^{\prime}}(-) H_{I}^{W}\right|_{n} ^{n-1}\right\}^{l^{\prime}} \frac{1}{H_{P, n-1}^{W+}-z_{n+1}^{\prime}} \times \\
& \times\left(\Gamma_{P, n-1}+\left.\Delta \Gamma_{P}\right|_{n} ^{n-1}\right)_{i^{\prime}} \times \\
& \times \sum_{l^{\prime \prime}=0}^{\infty} \oint_{\gamma_{P, n+1}} d w_{n+1}\left\{\left.\frac{1}{H_{P, n-1}^{W+}-w_{n+1}}(-) H_{I}^{W}\right|_{n} ^{n-1}\right\}^{l^{\prime \prime}} \frac{1}{H_{P, n-1}^{W+}-w_{n+1}} \phi_{P, n-1} .
\end{aligned}
$$

To manipulate this long expression we introduce some short-hand notations. We suppress the dependence on $P$ and $n$ since these parameters do not change within the relevant part of the arguments, and set

$$
\begin{aligned}
R & :=\frac{1}{H_{P, n-1}^{W+}-z_{n+1}}, \quad R^{\prime}:=\frac{1}{H_{P, n-1}^{W+}-z_{n+1}^{\prime}}, \quad R^{\prime \prime}:=\frac{1}{H_{P, n-1}^{W+}-w_{n+1}}, \\
R^{\prime \prime \prime} & :=\frac{1}{H_{P, n-1}^{W+}-w_{n+1}^{\prime}}, \quad V:=\left.(-) H_{I}^{W}\right|_{n} ^{n-1} .
\end{aligned}
$$

We also set

$$
\left(Q_{P, n-1}^{\perp}\right)^{q}= \begin{cases}Q_{P, n-1}^{\perp} & \text { for } q=0, \\ \sum_{r=1}^{\infty} \oint_{\gamma_{P, n+1}} d w_{n+1}^{\prime}\left\{R^{\prime \prime \prime} V\right\}^{r} R^{\prime \prime \prime} & \text { for } q=1 .\end{cases}
$$

Furthermore, we define for $q=0,1$

$$
\begin{aligned}
I^{q}:=\left(Q_{P, n-1}^{\perp}\right)^{q} \sum_{l=0}^{\infty}\{R V\}^{l} R\left(\Gamma_{P, n-1}+\left.\Delta \Gamma_{P}\right|_{n} ^{n-1}\right)_{i} \sum_{l^{\prime}=0}^{\infty}\left\{R^{\prime} V\right\}^{l^{\prime}} R^{\prime}\left(\Gamma_{P, n-1}+\left.\Delta \Gamma_{P}\right|_{n} ^{n-1}\right)_{i^{\prime}} \\
\quad \times \sum_{l^{\prime \prime}=0}^{\infty} \oint_{\gamma_{P, n+1}} d w_{n+1}\left\{R^{\prime \prime} V\right\}^{l^{\prime \prime}} R^{\prime \prime} \phi_{P, n-1},
\end{aligned}
$$

and note that (4.9) $=I^{0}-I^{1}$. Thus to conclude the inductive argument, it suffices to show that $\left\|I^{q}\right\| \leq c \sigma_{n-1}^{-2 \delta}=$ $\left(c \epsilon^{2 \delta}\right) \sigma_{n}^{-2 \delta}$ and then set $\epsilon(\delta)$ sufficiently small. We divide the argument into three cases studied in Subsections 4.2, 4.3, 4.4. This lengthy discussion is preceded by Subsection 4.1, which explains briefly the main novel ingredients of the proof. Without further notice, in the estimates below we assume the constraints in Remark 3.13 and use the results of Section 3. 


\subsection{Main novel ingredients of the proof}

The overall strategy of the inductive proof of Theorem 4.3 is similar to proving claim i) in Theorem 4.1, but there are several additional complications, which we would like to briefly explain in this subsection. To demonstrate various pitfalls of the present proof, we will state several plausible looking relations decorated by '?'. We believe that all these relations are incorrect (although we did not attempt to formally disprove them). We hope that the remarks below will convince the reader that the proof of Theorem 4.3 is more than just a tedious application of an existing method. Concerning notation, we warn the reader that from now on the symbol $O(\ldots)$ is also referred to operators and means that their norm $\|\ldots\|_{\mathcal{F}_{n}}$ is $O(\ldots)$.

\subsubsection{Singular terms $b^{*}\left(\left.g_{i}\right|_{n} ^{n-1}\right) \phi_{P, n-1}, B^{*}\left(\left.G\right|_{n} ^{n-1}\right) \phi_{P, n-1}$}

Let us consider the following contribution to (4.19), which will be studied systematically in the second part of the proof, in Subsection 4.3,

$$
I_{\mathbb{N}_{0}, \mathbb{N}, 0}^{q}:=\left(Q_{P, n-1}^{\perp}\right)^{q} \sum_{l=0}^{\infty}\{R V\}^{l} R\left(\Gamma_{P, n-1}+\left.\Delta \Gamma_{P}\right|_{n} ^{n-1}\right)_{i} \sum_{l^{\prime}=0}^{\infty}\left\{R^{\prime} V\right\}^{l^{\prime}}\left(R^{\prime} V\right) R^{\prime}\left(\Gamma_{P, n-1}+\left.\Delta \Gamma_{P}\right|_{n} ^{n-1}\right)_{i^{\prime}} \phi_{P, n-1} .
$$

More specifically, we would like to look at the following part

$$
\begin{aligned}
I_{\mathbb{N}_{0}, \mathbb{N}, 0}^{q} \ni\left(Q_{P, n-1}^{\perp}\right)^{q} \sum_{l=0}^{\infty}\{R V\}^{l} R\left(\Gamma_{P, n-1}\right)_{i} \sum_{l^{\prime}=0}^{\infty}\left\{R^{\prime} V\right\}^{l^{\prime}}\left(R^{\prime} V\right) R^{\prime}\left(\Gamma_{P, n-1}\right)_{i^{\prime}} \phi_{P, n-1} \\
\ni\left(Q_{P, n-1}^{\perp}\right)^{q} \sum_{l=0}^{\infty}\{R V\}^{l} R\left(\Gamma_{P, n-1}\right)_{i} \sum_{l^{\prime}=0}^{\infty}\left\{R^{\prime} V\right\}^{l^{\prime}} Q_{P, n-1}^{\perp}\left(R^{\prime} V\right) R^{\prime}\left(\Gamma_{P, n-1}\right)_{i^{\prime}} \phi_{P, n-1},
\end{aligned}
$$

where $\ni$ means that we simply dropped the terms which are irrelevant for the present informal discussion.

By Lemma 3.12 (cf. also (4.60) below) we have

$$
\sum_{l=0}^{\infty}\{R V\}^{l}=O(1), \quad R\left(\Gamma_{P, n-1}\right)_{i}=O\left(\sigma_{n+1}^{-1}\right), \quad \sum_{l^{\prime}=0}^{\infty}\left\{R^{\prime} V\right\}^{l^{\prime}}=O(1)
$$

Thus in order to obtain the desired bound, namely $(4.21)=O\left(\sigma_{n-1}^{-2 \delta}\right)$, we have to control the factor $R\left(\Gamma_{P, n-1}\right)_{i}$, for example by compensating the singularity $\sigma_{n+1}^{-1}$ appearing in the second estimate in (4.22). One might try to achieve this by establishing the following bound

$$
Q_{P, n-1}^{\perp}\left(R^{\prime} V\right) R^{\prime}\left(\Gamma_{P, n-1}\right)_{i^{\prime}} \phi_{P, n-1} \stackrel{?}{=} O\left(|\lambda|^{1 / 2} \sigma_{n-1}^{1-2 \delta}\right),
$$

and then using $|\lambda| \leq \epsilon^{8}$ to estimate $|\lambda|^{1 / 2} \sigma_{n+1}^{-1} \sigma_{n-1}^{1-2 \delta}=|\lambda|^{1 / 2} \epsilon^{2} \sigma_{n-1}^{-2 \delta} \leq \sigma_{n-1}^{-2 \delta}$. At first sight (4.23) may seem plausible: By Theorem 4.1 we have $R^{\prime}\left(\Gamma_{P, n-1}\right)_{i^{\prime}} \phi_{P, n-1}=O\left(\sigma_{n-1}^{-\delta}\right)$. Furthermore, by (3.46),

$$
-V:=\left.H_{I}^{W}\right|_{n} ^{n-1}=\left(\left.\mathcal{L}\right|_{n} ^{n-1}+\left.\mathcal{I}\right|_{n} ^{n-1}\right) \cdot \Gamma_{P, n-1}+\Delta\left(\left.H_{I}^{W}\right|_{n} ^{n-1}\right)_{\mathrm{mix}}+\left(\left.H_{I}^{W}\right|_{n} ^{n-1}\right)_{\mathrm{quad}},
$$

where by Lemma 3.12 and definition (3.28)

$$
R^{\prime} \Delta\left(\left.H_{I}^{W}\right|_{n} ^{n-1}\right)_{\mathrm{mix}}=O\left(|\lambda|^{1 / 2} \sigma_{n-1}\right), \quad R^{\prime}\left(\left.H_{I}^{W}\right|_{n} ^{n-1}\right)_{\text {quad }}=O\left(|\lambda| \sigma_{n-1}\right), \quad|\mathcal{I}|_{n}^{n-1} \mid \leq c_{I} \lambda^{2} \sigma_{n-1} .
$$

Given this information we are seemingly close to establishing (4.23), since we can write

$$
Q_{P, n-1}^{\perp}\left(R^{\prime} V\right) R^{\prime}\left(\Gamma_{P, n-1}\right)_{i^{\prime}} \phi_{P, n-1}=Q_{P, n-1}^{\perp} R^{\prime}\left(\left.\mathcal{L}\right|_{n} ^{n-1} \cdot \Gamma_{P, n-1}\right) R^{\prime}\left(\Gamma_{P, n-1}\right)_{i^{\prime}} \phi_{P, n-1}+O\left(|\lambda|^{1 / 2} \sigma_{n-1}^{1-2 \delta}\right),
$$


where we also used the induction hypothesis (4.6) to treat the term involving $\left.\mathcal{I}\right|_{n} ^{n-1}$.

Let us now pay close attention to the first term on the r.h.s. of (4.26), involving $\mathcal{L}_{n}^{n-1} \cdot \Gamma_{P, n-1}$. First, we recall the definition of $\left.\mathcal{L}\right|_{n} ^{n-1}$

$$
\left.\mathcal{L}\right|_{n} ^{n-1}:=-\lambda \int_{\left.\mathcal{A}\right|_{n} ^{n-1}} d^{3} k \frac{k\left(b(k)+b^{*}(k)\right)}{\sqrt{2}|k|^{3 / 2} \alpha_{P, n-1}(\hat{k})},
$$

which easily gives $\left.\mathcal{L}\right|_{n} ^{n-1} \phi_{P, n-1}=O\left(|\lambda|^{1 / 2} \sigma_{n-1}\right)$. One could hope that by combining this property with the inductive hypothesis (4.6) the desired bound follows, i.e., that the following implication holds

$$
\begin{gathered}
\left\{Q_{P, n-1}^{\perp} R^{\prime}\left(\Gamma_{P, n-1}\right)_{i} R^{\prime}\left(\Gamma_{P, n-1}\right)_{i^{\prime}} \phi_{P, n-1}=O\left(\sigma_{n-1}^{-2 \delta}\right),\left.\quad \mathcal{L}\right|_{n} ^{n-1} \phi_{P, n-1}=O\left(|\lambda|^{1 / 2} \sigma_{n-1}\right)\right\} \\
\Downarrow ? \\
Q_{P, n-1}^{\perp} R^{\prime}\left(\left.\mathcal{L}\right|_{n} ^{n-1} \cdot \Gamma_{P, n-1}\right) R^{\prime}\left(\Gamma_{P, n-1}\right)_{i^{\prime}} \phi_{P, n-1} \stackrel{?}{=} O\left(|\lambda|^{1 / 2} \sigma_{n-1}^{1-2 \delta}\right) .
\end{gathered}
$$

It turns out, however, that both the implication in (4.28)-(4.29) and the bounds in (4.29), (4.23) resist verification. The infrared regularity encoded in the (correct) estimates in (4.28) does not carry over by simple 'power counting' to the expression on the 1.h.s. of (4.29).

Let us now outline a correct treatment of the 1.h.s. of (4.29), whose complete discussion is given in Lemma 4.18 below. First, we note that only the creation part $\left(\left.\mathcal{L}\right|_{n} ^{n-1}\right)^{(+)}$has a non-zero contribution to (4.29), i.e. we can write

$$
Q_{P, n-1}^{\perp} R^{\prime}\left(\left.\mathcal{L}\right|_{n} ^{n-1} \cdot \Gamma_{P, n-1}\right) R^{\prime}\left(\Gamma_{P, n-1}\right)_{i^{\prime}} \phi_{P, n-1}=R^{\prime}\left(\left(\left.\mathcal{L}\right|_{n} ^{n-1}\right)^{(+)} \cdot \Gamma_{P, n-1}\right) R^{\prime}\left(\Gamma_{P, n-1}\right)_{i^{\prime}} \phi_{P, n-1},
$$

where we also observed that the vector on the r.h.s. of (4.30) is automatically in the range of $Q_{P, n-1}^{\perp}$. In contrast to (4.30), for the induction hypothesis in (4.28) the projection $Q_{P, n-1}^{\perp}$ is essential. Thus one could doubt the implication in (4.28)-(4.29) already at the present stage. To understand better why this implication is problematic, we compute, using the direct integral representations from Subsection 3.4,

$$
\begin{aligned}
R^{\prime}\left(\left(\left.\mathcal{L}\right|_{n} ^{n-1}\right)^{(+)} \cdot \Gamma_{P, n-1}\right) R^{\prime}\left(\Gamma_{P, n-1}\right)_{i^{\prime}} \phi_{P, n-1} & :=\frac{1}{H_{P, n-1}^{W+}-z_{n+1}^{\prime}}\left(\left(\left.\mathcal{L}\right|_{n} ^{n-1}\right)^{(+)} \cdot \Gamma_{P, n-1}\right) \frac{1}{H_{P, n-1}^{W+}-z_{n+1}^{\prime}}\left(\Gamma_{P, n-1}\right)_{i^{\prime}} \phi_{P, n-1} \\
\simeq- & \lambda \int_{\left.\mathcal{A}\right|_{n} ^{n-1}}^{{ }^{\oplus}} \frac{d^{3} k}{\sqrt{2}|k|^{3 / 2}} \frac{k_{i}}{\alpha_{P, n-1}(\hat{k})} \times \\
& \times \frac{1}{\left[H_{P, n-1}^{W+}\right]_{k}-z_{n+1}^{\prime}} Q_{P, n-1}^{\perp}\left(\Gamma_{P, n-1}\right)_{i} \frac{1}{H_{P, n-1}^{W+}-z_{n+1}^{\prime}}\left(\Gamma_{P, n-1}\right)_{i^{\prime}} \phi_{P, n-1} \\
& -\lambda \int_{\left.\mathcal{A}\right|_{n} ^{n-1}}^{\oplus \frac{d^{3} k}{\sqrt{2}|k|^{3 / 2}} \frac{k_{i}}{\alpha_{P, n-1}(\hat{k})} \times} \\
& \times \frac{1}{\left[H_{P, n-1}^{W+}\right]_{k}-z_{n+1}^{\prime}} Q_{P, n-1}\left(\Gamma_{P, n-1}\right)_{i} \frac{1}{H_{P, n-1}^{W+}-z_{n+1}^{\prime}}\left(\Gamma_{P, n-1}\right)_{i^{\prime}} \phi_{P, n-1},
\end{aligned}
$$

where in the second step we inserted $1=Q_{P, n-1}+Q_{P, n-1}^{\perp}$ inside the direct integral. The term in (4.32) can indeed be treated using the induction hypothesis and the structure of $\left.\mathcal{L}\right|_{n} ^{n-1}$ (as we hoped to treat the whole expression (4.31) in (4.28)-(4.29)). Using estimate (3.72), $\left|k_{i}\right| \leq \sigma_{n-1}$ and $|\lambda|^{1 / 2}|\ln \epsilon|^{1 / 2} \leq c$ we conclude that $(4.32)=O\left(|\lambda|^{1 / 2} \sigma_{n-1}^{1-2 \delta}\right)$. But we are still left with (4.33). A careful analysis in Lemma 4.18 shows that there is a function $\left.g_{i^{\prime}}\right|_{n} ^{n-1}$ with support in $\left.\overline{\mathcal{A}}\right|_{n} ^{n-1}$ s.t.

$$
\text { (4.33) }=b^{*}\left(\left.g_{i^{\prime}}\right|_{n} ^{n-1}\right) \phi_{P, n-1}+O\left(|\lambda|^{1 / 2} \sigma_{n-1}^{1-2 \delta}\right) \quad \text { and } \quad\left|g_{i^{\prime}}(k)\right|_{n}^{n-1}|\leq| \lambda \mid \frac{c}{|k|^{3 / 2}} \sigma_{n-1}^{-\delta},
$$


where $b^{*}\left(\left.g_{i^{\prime}}\right|_{n} ^{n-1}\right):=\left.\int d^{3} k g_{i^{\prime}}(k)\right|_{n} ^{n-1} b^{*}(k)$. As the estimate in (4.34) only gives $b^{*}\left(\left.g_{i^{\prime}}\right|_{n} ^{n-1}\right) \phi_{P, n-1}=O\left(|\lambda|^{1 / 2} \sigma_{n-1}^{-\delta}\right)$, we see no hope for establishing (4.29), (4.23). Instead, the first relation in (4.34) should be substituted back to (4.21) and treated using separate arguments (see Lemmas 4.19, 4.20 below) so that the desired estimate (4.21) $=O\left(\sigma_{n-1}^{-2 \delta}\right)$ is eventually established.

To conclude this discussion we remark that in the last part of the proof, in Subsection 4.4, also 'two-photon' singular terms of the following form appear

$$
B^{*}\left(\left.G\right|_{n} ^{n-1}\right) \phi_{P, n-1}:=\left.\int d^{3} k_{1} d^{3} k_{2} G\left(k_{1}, k_{2}\right)\right|_{n} ^{n-1} b^{*}\left(k_{1}\right) b^{*}\left(k_{2}\right) \phi_{P, n-1} .
$$

Here $\left.G\right|_{n} ^{n-1}$ is a function with support in $\left.\overline{\mathcal{A}}\right|_{n} ^{n-1} \times\left.\overline{\mathcal{A}}\right|_{n} ^{n-1}$ which satisfies the pointwise bound

$$
\left.\left|G\left(k_{1}, k_{2}\right)\right|_{n}^{n-1}|\leq c| \lambda\right|^{2}\left|k_{1}\right|^{-3 / 2}\left|k_{2}\right|^{-3 / 2} \sigma_{n-1}^{1-\delta} .
$$

This bound gives $B^{*}\left(\left.G\right|_{n} ^{n-1}\right) \phi_{P, n-1}=O\left(|\lambda| \sigma_{n-1}^{1-2 \delta}\right)$ which is less regular than the error term $O\left(|\lambda|^{1 / 2} \sigma_{n-1}^{2-2 \delta}\right)$ in relation (4.201) below, where (4.35) enters. The origin of (4.35), which will be briefly discussed in Subsection 4.1.2, is analogous to the origin of $b^{*}\left(\left.g_{i^{\prime}}\right|_{n} ^{n-1}\right) \phi_{P, n-1}$ above.

\subsubsection{Straight-line contours $\tilde{\gamma}_{P, n}$ and the maximal modulus principle}

Let us now consider the following contribution to (4.19), which will be studied systematically in the last part of the proof, in Subsection 4.4

$$
\begin{gathered}
I_{\mathbb{N}_{0}, \mathbb{N}_{0}, \mathbb{N}}^{q}:=\left(Q_{P, n-1}^{\perp}\right)^{q} \sum_{l=0}^{\infty}\{R V\}^{l} R\left(\Gamma_{P, n-1}+\left.\Delta \Gamma_{P}\right|_{n} ^{n-1}\right)_{i} \sum_{l^{\prime}=0}^{\infty}\left\{R^{\prime} V\right\}^{l^{\prime}} R^{\prime}\left(\Gamma_{P, n-1}+\left.\Delta \Gamma_{P}\right|_{n} ^{n-1}\right)_{i^{\prime}} \times \\
\times \sum_{l^{\prime \prime}=0}^{\infty} \oint_{\gamma_{P, n+1}} d w_{n+1}\left\{R^{\prime \prime} V\right\}^{l^{\prime \prime}} R^{\prime \prime} V R^{\prime \prime} \phi_{P, n-1} \\
\ni\left(Q_{P, n-1}^{\perp}\right)^{q} \sum_{l=0}^{\infty}\{R V\}^{l} R\left(\Gamma_{P, n-1}\right)_{i} \sum_{l^{\prime}=0}^{\infty}\left\{R^{\prime} V\right\}^{l^{\prime}} R^{\prime}\left(\Gamma_{P, n-1}\right)_{i^{\prime}} \times \\
\times \sum_{l^{\prime \prime}=0}^{\infty} \oint_{\gamma_{P, n+1}} d w_{n+1} f\left(w_{n+1}\right)\left\{R^{\prime \prime} V\right\}^{l^{\prime \prime}} Q_{P, n-1}^{\perp} R^{\prime \prime} V R^{\prime \prime} V \phi_{P, n-1} .
\end{gathered}
$$

Here in the second step we dropped the terms proportional to $\left.\Delta \Gamma_{P}\right|_{n} ^{n-1}$ and the $l^{\prime \prime}=0$ term in (4.37) as they will not be relevant for the present discussion. We also noticed that $R^{\prime \prime} \phi_{P, n-1}=f\left(w_{n+1}\right) \phi_{P, n-1}$, where the numerical function $f\left(w_{n+1}\right)=O\left(\sigma_{n+1}^{-1}\right)$ will be compensated by the length of the integration contour $\gamma_{P, n+1}$ in (4.38). We recall from (4.22) that simple-minded norm estimates of the factors $R\left(\Gamma_{P, n-1}\right)_{i}, R^{\prime}\left(\Gamma_{P, n-1}\right)_{i^{\prime}}$ yield together a singularity $O\left(\sigma_{n+1}^{-2}\right)$, which has to be tamed by a proper treatment of $Q_{P, n-1}^{\perp} R^{\prime \prime} V R^{\prime \prime} V \phi_{P, n-1}$. Lemma 4.25 gives the following estimate

$$
Q_{P, n-1}^{\perp} R^{\prime \prime} V R^{\prime \prime} V \phi_{P, n-1}=B^{*}\left(\left.G\right|_{n} ^{n-1}\right) \phi_{P, n-1}+\left(\left.\mathcal{I}\right|_{n} ^{n-1}\right)_{i^{\prime}} b^{*}\left(\left.g_{i^{\prime}}\right|_{n} ^{n-1}\right) \phi_{P, n-1}+O\left(|\lambda|^{1 / 2} \sigma_{n-1}^{2-2 \delta}\right),
$$

where the summation over $i^{\prime}=1,2,3$ is understood and the singular terms $B^{*}\left(\left.G\right|_{n} ^{n-1}\right) \phi_{P, n-1}, b^{*}\left(\left.g_{i^{\prime}}\right|_{n} ^{n-1}\right) \phi_{P, n-1}$ were discussed in Subsection 4.1.1. The error term $O\left(|\lambda|^{1 / 2} \sigma_{n-1}^{2-2 \delta}\right)$ compensates the $O\left(\sigma_{n+1}^{-2}\right)$ singularity directly, while the singular terms are substituted back to (4.38) and treated by separate arguments, in particular in Lemmas 4.28, 4.26. Eventually, the desired bound $(4.38)=O\left(\sigma_{n-1}^{-2 \delta}\right)$ is established.

In the remaining part of this subsection we will have a closer look at one particular contribution to the expression $Q_{P, n-1}^{\perp} R^{\prime \prime} V R^{\prime \prime} V \phi_{P, n-1}$, namely (cf. definition (4.24))

$$
R^{\prime \prime}\left(\left(\left.\mathcal{L}\right|_{n} ^{n-1}\right)^{(+)} \cdot \Gamma_{P, n-1}\right) R^{\prime \prime}\left(\left(\left.\mathcal{L}\right|_{n} ^{n-1}\right)^{(+)} \cdot \Gamma_{P, n-1}\right) \phi_{P, n-1},
$$


where we dropped the projection $Q_{P, n-1}^{\perp}$ as this vector is manifestly in its range. Making use of the direct integral representations from Subsection 3.4, and recalling that $R^{\prime \prime}=\left(H_{P, n-1}^{W+}-w_{n+1}\right)^{-1}$, we obtain

$$
\begin{aligned}
& (4.40) \simeq \frac{\lambda^{2}}{\sqrt{2}} \int_{\left(\mathcal{A l n} n^{n-1}\right)^{\times 2}}^{\oplus} d^{3} k_{1} d^{3} k_{2}\left(\frac{1}{\sqrt{2}\left|k_{1}\right|^{3 / 2}} \frac{k_{1, j}}{\alpha_{P, n-1}\left(\hat{k}_{1}\right)} \frac{1}{\sqrt{2}\left|k_{2}\right|^{3 / 2}} \frac{k_{2, j^{\prime}}}{\alpha_{P, n-1}\left(\hat{k}_{2}\right)} \times\right. \\
& \left.\times \frac{1}{\left[H_{P, n-1}^{W+}\right]_{k_{1}, k_{2}}-w_{n+1}}\left(\Gamma_{P, n-1}+k_{2}\right)_{j} \frac{1}{\left[H_{P, n-1}^{W+}\right]_{k_{2}}-w_{n+1}}\left(\Gamma_{P, n-1}\right)_{j^{\prime}} \phi_{P, n-1}+\{1 \leftrightarrow 2\}\right) \\
& \ni \frac{\lambda^{2}}{\sqrt{2}} \int_{\left(\left.\mathcal{A}\right|_{n} ^{n-1}\right)^{\times 2}}^{\oplus} d^{3} k_{1} d^{3} k_{2}\left(\frac{1}{\sqrt{2}\left|k_{1}\right|^{3 / 2}} \frac{k_{1, j}}{\alpha_{P, n-1}\left(\hat{k}_{1}\right)} \frac{1}{\sqrt{2}\left|k_{2}\right|^{3 / 2}} \frac{k_{2, j^{\prime}}}{\alpha_{P, n-1}\left(\hat{k}_{2}\right)} \times\right. \\
& \left.\times \frac{1}{\left[H_{P, n-1}^{W+}\right]_{k_{1}, k_{2}}-w_{n+1}} Q_{P, n-1}^{\perp}\left(\Gamma_{P, n-1}\right)_{j} \frac{1}{\left[H_{P, n-1}^{W+}\right]_{k_{2}}-w_{n+1}}\left(\Gamma_{P, n-1}\right)_{j^{\prime}} \phi_{P, n-1}+\{1 \leftrightarrow 2\}\right) \\
& +\frac{\lambda^{2}}{\sqrt{2}} \int_{\left(\left.\mathcal{A l}\right|_{n} ^{n-1}\right)^{\times 2}}^{\oplus} d^{3} k_{1} d^{3} k_{2}\left(\frac{1}{\sqrt{2}\left|k_{1}\right|^{3 / 2}} \frac{k_{1, j}}{\alpha_{P, n-1}\left(\hat{k}_{1}\right)} \frac{1}{\sqrt{2}\left|k_{2}\right|^{3 / 2}} \frac{k_{2, j^{\prime}}}{\alpha_{P, n-1}\left(\hat{k}_{2}\right)} \times\right. \\
& \left.\times \frac{1}{\left[H_{P, n-1}^{W+}\right]_{k_{1}, k_{2}}-w_{n+1}} Q_{P, n-1}\left(\Gamma_{P, n-1}\right)_{j} \frac{1}{\left[H_{P, n-1}^{W+}\right]_{k_{2}}-w_{n+1}}\left(\Gamma_{P, n-1}\right)_{j^{\prime}} \phi_{P, n-1}+\{1 \leftrightarrow 2\}\right),
\end{aligned}
$$

where in the second step we skipped the term proportional to $k_{2, j}$ from $\left(\Gamma_{P, n-1}+k_{2}\right)_{j}$ (as it will not be relevant for the present discussion) and inserted $1=Q_{P, n-1}+Q_{P, n-1}^{\perp}$ similarly as in (4.32)-(4.33). As one might expect from this latter computation, (4.43) gives rise to the singular term $B^{*}\left(\left.G\right|_{n} ^{n-1}\right) \phi_{P, n-1}$ in (4.39). Our main concern here is, however, the term in (4.42), which will eventually contribute to the error term $O\left(|\lambda|^{1 / 2} \sigma_{n-1}^{2-2 \delta}\right)$ in (4.39).

Our analysis of (4.42) starts from a declaration that the factor $\lambda^{2} k_{1, j} k_{2, j^{\prime}}$, which satisfies $\lambda^{2}\left|k_{1, j}\right|\left|k_{2, j}\right| \leq$ $\lambda^{2} \sigma_{n-1}^{2}$, should contribute the $|\lambda|^{1 / 2} \sigma_{n-1}^{2}$-part of the anticipated estimate $O\left(|\lambda|^{1 / 2} \sigma_{n-1}^{2-2 \delta}\right)$. Thus for the remaining part of (4.42) we need to establish a bound of order $\sigma^{-2 \delta}$, possibly multiplied by some inverse power of $\epsilon$. Given the form of this bound and the structure of this remaining part, it is natural to try to relate it to the inductive hypothesis (4.7).

To this end, we first use estimate (3.72) with $\phi:=\left(\left[H_{P, n-1}^{W+}\right]_{k_{2}}-w_{n+1}\right)^{-1}\left(\Gamma_{P, n-1}\right)_{j^{\prime}} \phi_{P, n-1}$, which gives for $\sigma_{n} \leq\left|k_{1}\right|,\left|k_{2}\right| \leq \sigma_{n-1}$

$$
\begin{aligned}
& \left\|\frac{1}{\left[H_{P, n-1}^{W+}\right]_{k_{1}, k_{2}}-w_{n+1}} Q_{P, n-1}^{\perp}\left(\Gamma_{P, n-1}\right)_{j} \frac{1}{\left[H_{P, n-1}^{W+}\right]_{k_{2}}-w_{n+1}}\left(\Gamma_{P, n-1}\right)_{j^{\prime}} \phi_{P, n-1}\right\| \\
& \leq c\left\|Q_{P, n-1}^{\perp} \frac{1}{H_{P, n-1}^{W+}-w_{n+1}}\left(\Gamma_{P, n-1}\right)_{j} \frac{1}{\left[H_{P, n-1}^{W+}\right]_{k_{2}}-w_{n+1}}\left(\Gamma_{P, n-1}\right)_{j^{\prime}} \phi_{P, n-1}\right\| .
\end{aligned}
$$

In Lemma 4.30 below we essentially replace $\left[H_{P, n-1}^{W+}\right]_{k_{2}}$ with $H_{P, n-1}^{W+}$ by a suitable expansion of the resolvent. To explain this argument, we first recall that

$$
\left[H_{P, n-1}^{W+}\right]_{k}:=H_{P, n-1}^{W+}+k \cdot \Gamma_{P, n-1}+\frac{|k|^{2}}{2}+\alpha_{P, n-1}(\hat{k})|k| .
$$

Next, we expand the resolvent of $\left[H_{P, n-1}^{W+}\right]_{k}$ in (4.44) to the second order:

$$
\begin{aligned}
\frac{1}{\left[H_{P, n-1}^{W+}\right]_{k}-w_{n+1}}= & \frac{1}{H_{P, n-1}^{W+}+\alpha_{P, n-1}(\hat{k})|k|-w_{n+1}} \\
& +\frac{1}{H_{P, n-1}^{W+}+\alpha_{P, n-1}(\hat{k})|k|-w_{n+1}}\left[-k \cdot \Gamma_{P, n-1}-\frac{|k|^{2}}{2}\right] \frac{1}{H_{P, n-1}^{W+}+\alpha_{P, n-1}(\hat{k})|k|-w_{n+1}} \\
& +\frac{1}{\left[H_{P, n-1}^{W+}\right]_{k}-w_{n+1}}\left\{\left[-k \cdot \Gamma_{P, n-1}-\frac{|k|^{2}}{2}\right] \frac{1}{H_{P, n-1}^{W+}+\alpha_{P, n-1}(\hat{k})|k|-w_{n+1}}\right\}^{2} .
\end{aligned}
$$


We set $\tilde{w}_{n+1}:=w_{n+1}-\alpha_{P, n-1}(\hat{k})|k|$ and consider the contribution of (4.46) to (4.44). We have

$$
\begin{aligned}
& \left\|Q_{P, n-1}^{\perp} \frac{1}{H_{P, n-1}^{W+}-w_{n+1}}\left(\Gamma_{P, n-1}\right)_{j} \frac{1}{H_{P, n-1}^{W+}-\tilde{w}_{n+1}}\left(\Gamma_{P, n-1}\right)_{j^{\prime}} \phi_{P, n-1}\right\| \\
& \leq \sup _{z_{n+1}^{\prime} \in \tilde{\gamma}_{P, n+1}}\left\|Q_{P, n-1}^{\perp} \frac{1}{H_{P, n-1}^{W+}-w_{n+1}}\left(\Gamma_{P, n-1}\right)_{j} \frac{1}{H_{P, n-1}^{W+}-z_{n+1}^{\prime}}\left(\Gamma_{P, n-1}\right)_{j^{\prime}} \phi_{P, n-1}\right\| \leq \sigma_{n-1}^{-2 \delta},
\end{aligned}
$$

where we used that $\alpha_{P, n-1}(\hat{k})|k| \geq 0$, applied the maximal modulus principle and the inductive hypothesis via relation (4.7). We refrain from a complete analysis of the higher order terms (4.47), (4.48) here, which give contributions to (4.44) of order $O\left(\epsilon^{-4} \sigma_{n-1}^{-2 \delta}\right)$ and eventually lead to (4.42) $=O\left(|\lambda|^{1 / 2} \sigma_{n-1}^{2-2 \delta}\right)$. However, we would like to remark for future reference that an alternative expansion, related to (4.46)-(4.48) by

$$
\begin{aligned}
\frac{1}{H_{P, n-1}^{W+}+\alpha_{P, n-1}(\hat{k})|k|-w_{n+1}} & \rightarrow \frac{1}{H_{P, n-1}^{W+}-w_{n+1}}, \\
{\left[-k \cdot \Gamma_{P, n-1}-\frac{|k|^{2}}{2}\right] } & \rightarrow\left[-k \cdot \Gamma_{P, n-1}-\alpha_{P, n-1}(\hat{k})|k|-\frac{|k|^{2}}{2}\right],
\end{aligned}
$$

generates higher order terms which, substituted to (4.44), do not appear to behave as $\sigma_{n-1}^{-2 \delta}$. Thus there is no obvious alternative to expansion (4.46)-(4.48) and the use of variable $\tilde{w}_{n+1}:=w_{n+1}-\alpha_{P, n-1}(\hat{k})|k|$ introduced above.

We would like to stress that the use of the straight-line contour $\tilde{\gamma}_{P, n+1}$ in the induction hypothesis (4.7) was essential for estimate (4.49). If instead of (4.7) we only had

$$
\sup _{w_{n+1}^{\prime}, w_{n+1}^{\prime \prime} \in \gamma_{P, n+1}}\left\|\frac{Q_{P, n-1}^{\perp}}{H_{P, n-1}^{W}-w_{n+1}^{\prime}}\left(\Gamma_{P, n-1}\right)_{i} \frac{1}{H_{P, n-1}^{W}-w_{n+1}^{\prime \prime}}\left(\Gamma_{P, n-1}\right)_{i^{\prime}} \phi_{P, n-1}\right\| \leq \sigma_{n-1}^{-2 \delta},
$$

we could not conclude. Indeed, for $w_{n+1} \in \gamma_{P, n+1}$ the resulting variable $\tilde{w}_{n+1}:=w_{n+1}-\alpha_{P, n-1}(\hat{k})|k|$ is always outside of the circle $\gamma_{P, n+1}$ and the maximal modulus principle does not apply. (Using the larger circle $\gamma_{P, n}$, in analogy to (4.6), does not help). A direct application of the Cauchy integral formula, which is a potential tool to handle such problems, does not apply for the same reason. Also a shift of the resolvent as in (4.50), (4.51) generates problematic error terms as mentioned above.

Finally we remark that for the induction hypothesis of Theorem 4.1 straight-line contours $\tilde{\gamma}_{P, n+1}$ were not essential. If instead of the induction hypothesis (4.4) we had

$$
\sup _{w_{n+1} \in \gamma_{P, n+1}}\left\|\frac{1}{H_{P, n-1}^{W+}-w_{n+1}}\left(\Gamma_{P, n-1}\right)_{i} \phi_{P, n-1}\right\| \leq \sigma_{n-1}^{-\delta},
$$

the induction would still close, yielding a weaker variant of Theorem 4.1 with $\tilde{\gamma}_{P, n+1}$ replaced with $\gamma_{P, n+1}$. (This variant would not suffice to prove Theorem 4.3, however).

\subsection{Contribution to $I^{q}$ with $l \in \mathbb{N}_{0}, l^{\prime}=0, l^{\prime \prime}=0$}

The contribution we analyse in this subsection is stated below. In (4.54)-(4.56) below we insert $Q_{P, n-1}^{\perp}+Q_{P, n-1}=$ 
1 and use $\sum_{l=0}^{\infty}\{R V\}^{l}=1+\sum_{l=0}^{\infty}\{R V\}^{l} R V$.

$$
\begin{aligned}
I_{\mathbb{N}_{0}, 0,0}^{q}:= & \left(Q_{P, n-1}^{\perp}\right)^{q} \sum_{l=0}^{\infty}\{R V\}^{l} R\left(\Gamma_{P, n-1}+\left.\Delta \Gamma_{P}\right|_{n} ^{n-1}\right)_{i} R^{\prime}\left(\Gamma_{P, n-1}+\left.\Delta \Gamma_{P}\right|_{n} ^{n-1}\right)_{i^{\prime}} \phi_{P, n-1} \\
= & \left(Q_{P, n-1}^{\perp}\right)^{q} \sum_{l=0}^{\infty}\{R V\}^{l} Q_{P, n-1}^{\perp} R\left(\Gamma_{P, n-1}\right)_{i} R^{\prime}\left(\Gamma_{P, n-1}\right)_{i^{\prime}} \phi_{P, n-1} \\
& +\delta_{q, 1}\left(Q_{P, n-1}^{\perp}\right)^{q} Q_{P, n-1} R\left(\Gamma_{P, n-1}\right)_{i} R^{\prime}\left(\Gamma_{P, n-1}\right)_{i^{\prime}} \phi_{P, n-1} \\
& +\left(Q_{P, n-1}^{\perp}\right)^{q} \sum_{l=0}^{\infty}\{R V\}^{l} R V Q_{P, n-1} R\left(\Gamma_{P, n-1}\right)_{i} R^{\prime}\left(\Gamma_{P, n-1}\right)_{i^{\prime}} \phi_{P, n-1} \\
& +\left(Q_{P, n-1}^{\perp}\right)^{q} \sum_{l=0}^{\infty}\{R V\}^{l} R\left(\left.\Delta \Gamma_{P}\right|_{n} ^{n-1}\right)_{i} R^{\prime}\left(\Gamma_{P, n-1}\right)_{i^{\prime}} \phi_{P, n-1} \\
& +\left(Q_{P, n-1}^{\perp}\right)^{q} \sum_{l=0}^{\infty}\{R V\}^{l} R\left(\Gamma_{P, n-1}\right)_{i} R^{\prime}\left(\left.\Delta \Gamma_{P}\right|_{n} ^{n-1}\right)_{i^{\prime}} \phi_{P, n-1} \\
& +\left(Q_{P, n-1}^{\perp}\right)^{q} \sum_{l=0}^{\infty}\{R V\}^{l} R\left(\left.\Delta \Gamma_{P}\right|_{n} ^{n-1}\right)_{i} R^{\prime}\left(\left.\Delta \Gamma_{P}\right|_{n} ^{n-1}\right)_{i^{\prime}} \phi_{P, n-1}=O\left(\sigma_{n-1}^{-2 \delta}\right) .
\end{aligned}
$$

To justify the last estimate we first recall that by (3.51) in Lemma 3.12

$$
\left\|\sum_{l=0}^{\infty}\{R V\}^{l}\right\|_{\mathcal{F}_{n}}:=\left\|\sum_{l=0}^{\infty}\left\{\left.\frac{1}{H_{P, n-1}^{W+}-z_{n+1}}(-) H_{I}^{W}\right|_{n} ^{n-1}\right\}^{l}\right\|_{\mathcal{F}_{n}} \leq \frac{1}{1-|\lambda|^{1 / 2} c_{2}} .
$$

Now (4.59) follows from Lemmas 4.6- 4.13 below.

Lemma 4.6. (Control of (4.54)) Under the assumptions of Theorem 4.3 and the inductive hypothesis

$$
\begin{aligned}
& Q_{P, n-1}^{\perp} R\left(\Gamma_{P, n-1}\right)_{i} R^{\prime}\left(\Gamma_{P, n-1}\right)_{i^{\prime}} \phi_{P, n-1} \\
& :=Q_{P, n-1}^{\perp} \frac{1}{H_{P, n-1}^{W+}-z_{n+1}}\left(\Gamma_{P, n-1}\right)_{i} \frac{1}{H_{P, n-1}^{W+}-z_{n+1}^{\prime}}\left(\Gamma_{P, n-1}\right)_{i^{\prime}} \phi_{P, n-1}=O\left(\sigma_{n-1}^{-2 \delta}\right) .
\end{aligned}
$$

Proof. This is a consequence of the inductive hypothesis stated in (4.7).

Lemma 4.7. (Control of (4.55)) Under the assumptions of Theorem 4.3 and for $q=1$

$$
\begin{aligned}
& \delta_{q, 1}\left(Q_{P, n-1}^{\perp}\right)^{q} Q_{P, n-1} \\
& :=\sum_{r=1}^{\infty} \oint_{\gamma_{P, n+1}} d w_{n+1}^{\prime}\left\{\left.\frac{1}{H_{P, n-1}^{W+}-w_{n+1}^{\prime}}(-) H_{I}^{W}\right|_{n} ^{n-1}\right\}^{r} \frac{1}{H_{P, n-1}^{W+}-w_{n+1}^{\prime}} Q_{P, n-1}=O\left(|\lambda|^{1 / 2} \sigma_{n-1}^{1-\delta}\right) .
\end{aligned}
$$

Proof. We first note that

$$
\frac{1}{H_{P, n-1}^{W+}-w_{n+1}^{\prime}} Q_{P, n-1}=f\left(w_{n+1}^{\prime}\right) Q_{P, n-1},
$$

where $f\left(w_{n+1}^{\prime}\right)=O\left(\sigma_{n+1}^{-1}\right)$. Furthermore, by Lemma 4.10 below

$$
\left.\frac{1}{H_{P, n-1}^{W+}-w_{n+1}^{\prime}}(-) H_{I}^{W}\right|_{n} ^{n-1} Q_{P, n-1}=O\left(|\lambda|^{1 / 2} \sigma_{n-1}^{1-\delta}\right)
$$


Thus taking into account that by Lemma 3.12

$$
\left\|\left.\frac{1}{H_{P, n-1}^{W+}-w_{n+1}^{\prime}}(-) H_{I}^{W}\right|_{n} ^{n-1}\right\|_{\mathcal{F}_{n}}=O\left(|\lambda|^{1 / 2}\right),
$$

and that the length of the region of integration is proportional to $\sigma_{n+1}$, we have

$$
\delta_{q, 1}\left(Q_{P, n-1}^{\perp}\right)^{q} Q_{P, n-1}=O\left(|\lambda|^{1 / 2} \sigma_{n-1}^{1-\delta}\right) .
$$

This concludes the proof.

Lemma 4.8. (Control of (4.55) and (4.56)) Under the assumptions of Theorem 4.3

$$
\begin{aligned}
& Q_{P, n-1} R\left(\Gamma_{P, n-1}\right)_{i} R^{\prime}\left(\Gamma_{P, n-1}\right)_{i^{\prime}} \phi_{P, n-1} \\
& :=Q_{P, n-1} \frac{1}{H_{P, n-1}^{W+}-z_{n+1}}\left(\Gamma_{P, n-1}\right)_{i} \frac{1}{H_{P, n-1}^{W+}-z_{n+1}^{\prime}}\left(\Gamma_{P, n-1}\right)_{i^{\prime}} \phi_{P, n-1}=O\left(\sigma_{n+1}^{-1} \sigma_{n-1}^{-\delta}\right) .
\end{aligned}
$$

Proof. Clearly, the expression from the statement of the lemma is bounded in norm by

$$
\frac{c_{0}}{\sigma_{n+1}}\left\|Q_{P, n-1}\left(\Gamma_{P, n-1}\right)_{i}\right\|_{\mathcal{F}_{n-1}}\left\|\frac{1}{H_{P, n-1}^{W+}-z_{n+1}^{\prime}}\left(\Gamma_{P, n-1}\right)_{i^{\prime}} \phi_{P, n-1}\right\| \leq \frac{c_{0}}{\sigma_{n+1}}\left\|Q_{P, n-1}\left(\Gamma_{P, n-1}\right)_{i}\right\|_{\mathcal{F}_{n-1}} \frac{1}{\sigma_{n-1}^{\delta}},
$$

where we applied estimate (3.47) of Lemma 3.12 and Theorem 4.1 (more precisely second formula in (4.4)). To conclude the proof we note that (cf. formulas (3.21), (3.38), and (3.8))

$$
\left\|Q_{P, n-1}\left(\Gamma_{P, n-1}\right)_{i}\right\|_{\mathcal{F}_{n-1}} \leq c+\left\|\bar{Q}_{P, n-1}\left(P-P_{\mathrm{f}}\right)_{i}\right\|_{\mathcal{F}_{n-1}} \leq c+c^{\prime}\left\|\bar{Q}_{P, n-1} H_{P, \text { free }} \bar{Q}_{P, n-1}\right\|_{\mathcal{F}_{n-1}}^{1 / 2},
$$

which is bounded uniformly in $n$ by Lemma A.3.

Remark 4.9. Without further notice, in the next lemmas we repeatedly make use of the constraint $|\lambda|^{1 / 2} \leq \epsilon^{4}$.

Lemma 4.10. (Control of (4.56)) Under the assumptions of Theorem 4.3

$$
R V Q_{P, n-1}:=\left.\frac{1}{H_{P, n-1}^{W+}-z_{n+1}}(-) H_{I}^{W}\right|_{n} ^{n-1} Q_{P, n-1}=O\left(|\lambda|^{1 / 2} \sigma_{n-1}^{1-\delta}\right) .
$$

The same bound is true for $z_{n+1} \in \gamma_{P, n+1}$.

Proof. We recall from (3.46) that

$$
\left.H_{I}^{W}\right|_{n} ^{n-1}:=\left(\left.\mathcal{L}\right|_{n} ^{n-1}+\left.\mathcal{I}\right|_{n} ^{n-1}\right) \cdot \Gamma_{P, n-1}+\Delta\left(\left.H_{I}^{W}\right|_{n} ^{n-1}\right)_{\text {mix }}+\left(\left.H_{I}^{W}\right|_{n} ^{n-1}\right)_{\text {quad }} .
$$

We consider the contributions from the respective terms on the r.h.s. of (4.71) to the expression in the statement of the lemma. First, making use of Lemma 4.15, we obtain

$$
\left.\frac{1}{H_{P, n-1}^{W+}-z_{n+1}} \mathcal{L}\right|_{n} ^{n-1} \cdot \Gamma_{P, n-1} Q_{P, n-1}=O\left(|\lambda||\ln \epsilon|^{1 / 2} \sigma_{n-1}^{1-\delta}\right) .
$$

Next, we have by (3.28)

$$
\left.|\mathcal{I}|_{n}^{n-1}\left|\leq c_{I}\right| \lambda\right|^{2} \sigma_{n-1}
$$

Thus we can write, applying Theorem 4.1,

$$
\left.\frac{1}{H_{P, n-1}^{W+}-z_{n+1}} \mathcal{I}\right|_{n} ^{n-1} \cdot \Gamma_{P, n-1} Q_{P, n-1}=O\left(|\lambda|^{2} \sigma_{n-1} \sigma_{n-1}^{-\delta}\right) .
$$

Finally, by Lemma 3.12,

$$
\frac{1}{H_{P, n-1}^{W+}-z_{n+1}}\left(\Delta\left(\left.H_{I}^{W}\right|_{n} ^{n-1}\right)_{\text {mix }}+\left(\left.H_{I}^{W}\right|_{n} ^{n-1}\right)_{\text {quad }}\right) Q_{P, n-1}=O\left(|\lambda|^{1 / 2} \sigma_{n-1}\right) . \square
$$


Lemma 4.11. (Control of (4.57)) Under the assumptions of Theorem 4.3

$$
R\left(\left.\Delta \Gamma_{P}\right|_{n} ^{n-1}\right)_{i} R^{\prime}\left(\Gamma_{P, n-1}\right)_{i^{\prime}} \phi_{P, n-1}=\frac{1}{H_{P, n-1}^{W+}-z_{n+1}}\left(\left.\Delta \Gamma_{P}\right|_{n} ^{n-1}\right)_{i} \frac{1}{H_{P, n-1}^{W+}-z_{n+1}^{\prime}}\left(\Gamma_{P, n-1}\right)_{i^{\prime}} \phi_{P, n-1}=O\left(\sigma_{n-1}^{-2 \delta}\right) .
$$

Proof. We recall that

$$
\left.\Delta \Gamma_{P}\right|_{n} ^{n-1}:=-\nabla E_{P, n-1}+\nabla E_{P, n}+\left.\mathcal{I}\right|_{n} ^{n-1}+\left.\mathcal{L}\right|_{n} ^{n-1} .
$$

Making use of the facts that (cf. (3.19), claims ii), iii) of Theorem 4.1 and (3.28))

$$
\left|\nabla E_{P, n-1}-\nabla E_{P, n}\right| \leq c_{1}\left[\lambda^{2} \sigma_{n-1}+4|\lambda|^{1 / 4} \sigma_{n-1}^{1-\delta}\right],\left.\quad|\mathcal{I}|_{n}^{n-1}\left|\leq c_{I}\right| \lambda\right|^{2} \sigma_{n-1},
$$

we have

$$
\left.\left|-\nabla E_{P, n-1}+\nabla E_{P, n}+\mathcal{I}\right|_{n}^{n-1}|\leq c| \lambda\right|^{1 / 4} \sigma_{n-1}^{1-\delta} .
$$

Now, by Theorem 4.1, we can write

$$
\begin{array}{r}
\frac{1}{H_{P, n-1}^{W+}-z_{n+1}}\left(-\nabla E_{P, n-1}+\nabla E_{P, n}+\left.\mathcal{I}\right|_{n} ^{n-1}\right)_{i} \frac{1}{H_{P, n-1}^{W+}-z_{n+1}^{\prime}}\left(\Gamma_{P, n-1}\right)_{i^{\prime}} \phi_{P, n-1} \\
=O\left(|\lambda|^{1 / 4} \sigma_{n+1}^{-1} \sigma_{n-1}^{1-\delta} \sigma_{n-1}^{-\delta}\right)=O\left(\sigma_{n-1}^{-2 \delta}\right) .
\end{array}
$$

Arguing similarly and making use of (3.50) in Lemma 3.12, we get

$$
\frac{1}{H_{P, n-1}^{W+}-z_{n+1}}\left(\left.\mathcal{L}\right|_{n} ^{n-1}\right)_{i} \frac{1}{H_{P, n-1}^{W+}-z_{n+1}^{\prime}}\left(\Gamma_{P, n-1}\right)_{i^{\prime}} \phi_{P, n-1}=O\left(|\lambda|^{1 / 2} \sigma_{n-1}^{-\delta}\right) .
$$

This concludes the proof.

Lemma 4.12. (Control of (4.58)) Under the assumptions of Theorem 4.3

$$
R\left(\Gamma_{P, n-1}\right)_{i} R^{\prime}\left(\left.\Delta \Gamma_{P}\right|_{n} ^{n-1}\right)_{i^{\prime}} \phi_{P, n-1}:=\frac{1}{H_{P, n-1}^{W+}-z_{n+1}}\left(\Gamma_{P, n-1}\right)_{i} \frac{1}{H_{P, n-1}^{W+}-z_{n+1}^{\prime}}\left(\left.\Delta \Gamma_{P}\right|_{n} ^{n-1}\right)_{i^{\prime}} \phi_{P, n-1}=O\left(\sigma_{n-1}^{-2 \delta}\right) .
$$

Proof. We have $\left.\Delta \Gamma_{P}\right|_{n} ^{n-1}:=-\nabla E_{P, n-1}+\nabla E_{P, n}+\left.\mathcal{I}\right|_{n} ^{n-1}+\left.\mathcal{L}\right|_{n} ^{n-1}$ and we consider first the contribution of the first three terms. Arguing as in the proof of Lemma 4.11, we have

$$
\begin{array}{r}
\frac{1}{H_{P, n-1}^{W+}-z_{n+1}}\left(\Gamma_{P, n-1}\right)_{i} \frac{1}{H_{P, n-1}^{W+}-z_{n+1}^{\prime}}\left(-\nabla E_{P, n-1}+\nabla E_{P, n}+\left.\mathcal{I}\right|_{n} ^{n-1}\right)_{i^{\prime}} \phi_{P, n-1} \\
=O\left(|\lambda|^{1 / 4} \sigma_{n-1}^{1-\delta} \sigma_{n+1}^{-1} \sigma_{n-1}^{-\delta}\right)=O\left(\sigma_{n-1}^{-2 \delta}\right) .
\end{array}
$$

Now we consider the contribution proportional to $\left.\mathcal{L}\right|_{n} ^{n-1}$. By Lemma 4.16 we have

$$
\frac{1}{H_{P, n-1}^{W+}-z_{n+1}}\left(\Gamma_{P, n-1}\right)_{i} \frac{1}{H_{P, n-1}^{W+}-z_{n+1}^{\prime}}\left(\left.\mathcal{L}\right|_{n} ^{n-1}\right)_{i^{\prime}} \phi_{P, n-1}=O\left(|\lambda|^{1 / 2} \sigma_{n-1}^{-\delta}\right),
$$

which concludes the proof.

Lemma 4.13. (Control of l.h.s.(4.59)) Under the assumptions of Theorem 4.3

$$
R\left(\left.\Delta \Gamma_{P}\right|_{n} ^{n-1}\right)_{i} R^{\prime}\left(\left.\Delta \Gamma_{P}\right|_{n} ^{n-1}\right)_{i^{\prime}} \phi_{P, n-1}:=\frac{1}{H_{P, n-1}^{W+}-z_{n+1}}\left(\left.\Delta \Gamma_{P}\right|_{n} ^{n-1}\right)_{i} \frac{1}{H_{P, n-1}^{W+}-z_{n+1}^{\prime}}\left(\left.\Delta \Gamma_{P}\right|_{n} ^{n-1}\right)_{i^{\prime}} \phi_{P, n-1}=O\left(\sigma_{n-1}^{-2 \delta}\right)
$$


Proof. Recalling from (4.79) that $\left.\Delta \Gamma_{P}\right|_{n} ^{n-1}=\left.X\right|_{n} ^{n-1}+\left.\mathcal{L}\right|_{n} ^{n-1}$, where $\left.X\right|_{n} ^{n-1}:=-\nabla E_{P, n-1}+\nabla E_{P, n}+\left.\mathcal{I}\right|_{n} ^{n-1}$ is a vector in $\mathbb{R}^{3}$ satisfying

$$
\left.|X|_{n}^{n-1}|\leq c| \lambda\right|^{1 / 4} \sigma_{n-1}^{1-\delta}
$$

we write

$$
\begin{aligned}
\text { 1.h.s.(4.85) }= & \frac{1}{H_{P, n-1}^{W+}-z_{n+1}}\left(\left.X\right|_{n} ^{n-1}\right)_{i} \frac{1}{H_{P, n-1}^{W+}-z_{n+1}^{\prime}}\left(\left.X\right|_{n} ^{n-1}\right)_{i^{\prime}} \phi_{P, n-1} \\
& +\frac{1}{H_{P, n-1}^{W+}-z_{n+1}}\left(\left.X\right|_{n} ^{n-1}\right)_{i} \frac{1}{H_{P, n-1}^{W+}-z_{n+1}^{\prime}}\left(\left.\mathcal{L}\right|_{n} ^{n-1}\right)_{i^{\prime}} \phi_{P, n-1} \\
& +\frac{1}{H_{P, n-1}^{W+}-z_{n+1}}\left(\left.\mathcal{L}\right|_{n} ^{n-1}\right)_{i} \frac{1}{H_{P, n-1}^{W+}-z_{n+1}^{\prime}}\left(\left.X\right|_{n} ^{n-1}\right)_{i^{\prime}} \phi_{P, n-1} \\
& +\frac{1}{H_{P, n-1}^{W+}-z_{n+1}}\left(\left.\mathcal{L}\right|_{n} ^{n-1}\right)_{i}^{(+)} \frac{1}{H_{P, n-1}^{W+}-z_{n+1}^{\prime}}\left(\left.\mathcal{L}\right|_{n} ^{n-1}\right)_{i^{\prime}} \phi_{P, n-1} . \\
& +\frac{1}{H_{P, n-1}^{W+}-z_{n+1}}\left(\left.\mathcal{L}\right|_{n} ^{n-1}\right)_{i}^{(-)} \frac{1}{H_{P, n-1}^{W+}-z_{n+1}^{\prime}}\left(\left.\mathcal{L}\right|_{n} ^{n-1}\right)_{i^{\prime}} \phi_{P, n-1},
\end{aligned}
$$

where $\left(\left.\mathcal{L}\right|_{n} ^{n-1}\right)_{i}^{( \pm)}$are the creation/annihilation parts of $\left(\left.\mathcal{L}\right|_{n} ^{n-1}\right)_{i}$. We have by (4.86) and Lemma 3.12

$$
\|(4.87)\| \leq c|\lambda|^{1 / 2}\left(\frac{\sigma_{n-1}}{\sigma_{n+1}}\right)^{2} \frac{1}{\sigma_{n-1}^{2 \delta}} \leq \frac{c^{\prime}}{\sigma_{n-1}^{2 \delta}} .
$$

Now we analyse the terms involving $\left.\mathcal{L}\right|_{n} ^{n-1}$. Clearly,

$$
(4.88)=-\lambda \int_{\left.\mathcal{A}\right|_{n} ^{n-1}}^{\oplus} \frac{d^{3} k}{\sqrt{2}|k|^{3 / 2}} \frac{k_{i^{\prime}}}{\alpha_{P, n-1}(\hat{k})} \frac{1}{\left[H_{P, n-1}^{W+}\right]_{k}-z_{n+1}} \frac{\left(\left.X\right|_{n} ^{n-1}\right)_{i}}{\left[H_{P, n-1}^{W+}\right]_{k}-z_{n+1}^{\prime}} \phi_{P, n-1},
$$

and therefore, by Lemma 3.14, (which gives $\left.\left|k_{i^{\prime}}\right|\left\|\left(\left[H_{P, n-1}^{W+}\right]_{k}-z_{n+1}\right)^{-1}\right\|_{\mathcal{F}_{n}}\left\|\left(\left[H_{P, n-1}^{W+}\right]_{k}-z_{n+1}^{\prime}\right)^{-1}\right\|_{\mathcal{F}_{n}} \leq c|k|^{-1}\right)$, $|k|^{-1} \leq \sigma_{n}^{-1}$ and estimate (4.86), we have

$$
\|(4.88)\| \leq c|\lambda|^{5 / 4} \sigma_{n-1}^{1-\delta}|\ln \epsilon|^{1 / 2} \sigma_{n}^{-1} \leq c|\lambda|^{5 / 4} \frac{\sigma_{n-1}}{\sigma_{n}}|\ln \epsilon|^{1 / 2} \frac{1}{\sigma_{n-1}^{\delta}} \leq \frac{c^{\prime}}{\sigma_{n-1}^{\delta}} .
$$

Now we consider (4.89). In the direct integral notation we have

$$
(4.89) \simeq-\lambda \int_{\left.\mathcal{A}\right|_{n} ^{n-1}}^{\oplus} \frac{d^{3} k}{\sqrt{2}|k|^{3 / 2}} \frac{k_{i}}{\alpha_{P, n-1}(\hat{k})} \frac{1}{\left[H_{P, n-1}^{W+}\right]_{k}-z_{n+1}} \frac{\left(\left.X\right|_{n} ^{n-1}\right)_{i^{\prime}}}{H_{P, n-1}^{W+}-z_{n+1}^{\prime}} \phi_{P, n-1} .
$$

by Lemma 3.14 and (4.86) we obtain

$$
\|(4.89)\| \leq c|\lambda|^{5 / 4} \frac{\sigma_{n-1}}{\sigma_{n+1}}|\ln \epsilon|^{1 / 2} \frac{1}{\sigma_{n-1}^{\delta}} \leq \frac{c^{\prime}}{\sigma_{n-1}^{\delta}} .
$$

Next, we consider (4.90). In the direct integral representation it has the form

$$
\begin{aligned}
(4.90) \simeq & \frac{\lambda^{2}}{\sqrt{2}} \int_{\left(\left.\mathcal{A}\right|_{n} ^{n-1}\right)^{\times 2}}^{\oplus} d^{3} k_{1} d^{3} k_{2} \frac{1}{\left[H_{P, n-1}^{W+}\right]_{k_{1}, k_{2}}-z_{n+1}} \times \\
& \times\left\{\frac{1}{\sqrt{2}\left|k_{1}\right|^{3 / 2}} \frac{k_{1, i}}{\alpha_{P, n-1}\left(\hat{k}_{1}\right)} \frac{1}{\sqrt{2}\left|k_{2}\right|^{3 / 2}} \frac{k_{2, i^{\prime}}}{\alpha_{P, n-1}\left(\hat{k}_{2}\right)} \frac{1}{\left[H_{P, n-1}^{W+}\right]_{k_{2}}-z_{n+1}} \phi_{P, n-1}+\{1 \leftrightarrow 2\}\right\} .
\end{aligned}
$$


Now making use of Lemma 3.14 we get $\|(4.90)\| \leq c|\lambda|^{2}|\ln \epsilon| \leq c^{\prime}$. Finally we estimate (4.91). It is easy to see that

$$
\begin{array}{r}
(4.91)=\lambda^{2} \frac{1}{H_{P, n-1}^{W+}-z_{n+1}} \int_{\left.\mathcal{A}\right|_{n} ^{n-1}} d^{3} k \frac{1}{2|k|^{3}} \frac{k_{i} k_{i^{\prime}}}{\alpha_{P, n-1}(\hat{k})^{2}} \frac{1}{\left[H_{P, n-1}^{W+}\right]_{k}-z_{n+1}^{\prime}} \phi_{P, n-1} \\
=O\left(\lambda^{2}|\ln \epsilon| \sigma_{n-1} \sigma_{n+1}^{-1}\right)=O(|\lambda|),
\end{array}
$$

where we made use of Lemma 3.12 and (3.70). This concludes the proof.

Remark 4.14. In the estimate of (4.94) and other direct integral estimates in the proof of Lemma 4.13, we follow a procedure that is not optimal but is enough for our purposes and will be applied (without further notice) to similar quantities studied in the rest of this paper. Namely, we first took the norm of the expression with the direct integral in order to obtain an ordinary integral, then used $\left|k_{i^{\prime}}\right|$ appearing in the numerator to compensate $1 /|k|$ resulting from the bound $\left\|\frac{1}{\left[H_{P, n-1}^{W+}\right]_{k}-z_{n+1}}\right\|_{\mathcal{F}_{n}} \leq \frac{c}{|k|}$ of Lemma 3.14, and only in the end computed the integral. (If we estimated instead $\frac{c}{|k|} \leq \frac{c}{\sigma_{n+1}}$, and computed the integral directly, incorporating $k_{i}^{\prime}$, we could avoid the factor $|\ln \epsilon|^{1 / 2}$ ). On other occasions, e.g. in the estimate (4.100), we apply $\left|k_{i}\right| \leq \sigma_{n-1}$ to the $k_{i}$ in the numerator. Again, the logarithms could be avoided here by streamlining the computation differently.

Lemma 4.15. Under the assumptions of Theorem 4.3 we have

$$
\left.\frac{1}{H_{P, n-1}^{W+}-z_{n+1}} \mathcal{L}\right|_{n} ^{n-1} \cdot \Gamma_{P, n-1} \phi_{P, n-1}=O\left(|\lambda \| \ln \epsilon|^{1 / 2} \sigma_{n-1}^{1-\delta}\right)
$$

for $z_{n+1} \in \gamma_{P, n+1} \cup \tilde{\gamma}_{P, n+1}$.

Proof. Making use of the direct integral representations from Subsection 3.4, we get

$$
\begin{aligned}
\left.\frac{1}{H_{P, n-1}^{W+}-z_{n+1}} \mathcal{L}\right|_{n} ^{n-1} \cdot \Gamma_{P, n-1} \phi_{P, n-1} & \simeq-\lambda \int_{\left.\mathcal{A}\right|_{n} ^{n-1}}^{\oplus} \frac{d^{3} k}{\sqrt{2}|k|^{3 / 2}} \frac{k_{i}}{\alpha_{P, n-1}(\hat{k})} \frac{1}{\left[H_{P, n-1}^{W+}\right]_{k}-z_{n+1}} \Gamma_{P, n-1} \phi_{P, n-1} \\
& =O\left(|\lambda \| \ln \epsilon|^{1 / 2} \sigma_{n-1}^{1-\delta}\right),
\end{aligned}
$$

where we applied (3.72) and Theorem 4.1. Here, the reader should note that (3.72) is used together with the identity

$$
\frac{1}{\left[H_{P, n-1}^{W+}\right]_{k}-z_{n+1}} \Gamma_{P, n-1} \phi_{P, n-1}=\frac{1}{\left[H_{P, n-1}^{W+}\right]_{k}-z_{n+1}} Q_{P, n-1}^{\perp} \Gamma_{P, n-1} \phi_{P, n-1}
$$

which follows from the definition in (3.21).

Lemma 4.16. Under the assumptions of Theorem 4.3 we have

$$
\begin{aligned}
& \frac{1}{H_{P, n-1}^{W+}-z_{n+1}}\left(\Gamma_{P, n-1}\right)_{i} \frac{1}{H_{P, n-1}^{W+}-z_{n+1}^{\prime}}\left(\left.\mathcal{L}\right|_{n} ^{n-1}\right)_{i^{\prime}} \phi_{P, n-1}=O\left(|\lambda|^{1 / 2} \sigma_{n-1}^{-\delta}\right), \\
& \frac{1}{H_{P, n-1}^{W+}-z_{n+1}}\left(\Gamma_{P, n-1}\right)_{i} \frac{1}{H_{P, n-1}^{W+}-z_{n+1}^{\prime}}\left(\left.\stackrel{\circ}{\mathcal{L}}\right|_{n} ^{n-1}\right) \phi_{P, n-1}=O\left(|\lambda|^{1 / 2} \sigma_{n-1}^{1-\delta}\right),
\end{aligned}
$$

for $z_{n+1}, z_{n+1}^{\prime} \in \gamma_{P, n+1} \cup \tilde{\gamma}_{P, n+1}$. Here we denoted

$$
\left(\stackrel{\mathcal{L}}{\left.\right|_{n} ^{n-1}}\right):=\sum_{i=1}^{3}\left[\left(\Gamma_{P, n-1}\right)_{i},\left(\left.\mathcal{L}\right|_{n} ^{n-1}\right)_{i}\right]=\lambda \int_{\sigma_{n}}^{\sigma_{n-1}} d^{3} k \frac{k^{2}\left(b(k)-b^{*}(k)\right)}{\sqrt{2}|k|^{3 / 2} \alpha_{P, n-1}(\hat{k})} .
$$


Proof. Making use of the direct integral representation from Subsection 3.4, we have

$$
\begin{aligned}
& \frac{1}{H_{P, n-1}^{W+}-z_{n+1}}\left(\Gamma_{P, n-1}\right)_{i} \frac{1}{H_{P, n-1}^{W+}-z_{n+1}^{\prime}}\left(\left.\mathcal{L}\right|_{n} ^{n-1}\right)_{i^{\prime}} \phi_{P, n-1} \\
& \simeq-\lambda \int_{\mathcal{P l}_{n}^{n-1}}^{\oplus} \frac{d^{3} k}{\sqrt{2}|k|^{3 / 2}} \frac{k_{i^{\prime}}}{\alpha_{P, n-1}(\hat{k})} \frac{1}{\left[H_{P, n-1}^{W+}\right]_{k}-z_{n+1}}\left(\Gamma_{P, n-1}+k\right)_{i} \frac{1}{\left[H_{P, n-1}^{W+}\right]_{k}-z_{n+1}^{\prime}} \phi_{P, n-1} \\
& =-\lambda \int_{\left.\mathcal{F}\right|_{n} ^{n-1}}^{\oplus} \frac{d^{3} k}{\sqrt{2}|k|^{3 / 2}} \frac{k_{i^{\prime}}}{\alpha_{P, n-1}(\hat{k})} \frac{1}{\left[H_{P, n-1}^{W+}\right]_{k}-z_{n+1}}\left(\Gamma_{P, n-1}\right)_{i} \frac{1}{\left[H_{P, n-1}^{W+}\right]_{k}-z_{n+1}^{\prime}} \phi_{P, n-1} \\
& +O\left(|\lambda \| \ln \epsilon|^{1 / 2}\right),
\end{aligned}
$$

where in the first step we made use of (3.62) and in the last step we applied (3.70). Since $|\lambda| \leq \epsilon^{8}$, the error term in (4.106) contributes to the r.h.s. of (4.102). To treat (4.105), we apply the following resolvent expansion to the resolvent next to $\phi_{P, n-1}$ :

$$
\begin{aligned}
\frac{1}{\left[H_{P, n-1}^{W+}\right]_{k}-z_{n+1}^{\prime}}= & \frac{1}{H_{P, n-1}^{W+}+\alpha_{P, n-1}(\hat{k})|k|-z_{n+1}^{\prime}} \\
& +\frac{1}{\left[H_{P, n-1}^{W+}\right]_{k}-z_{n+1}^{\prime}}\left[-k \cdot \Gamma_{P, n-1}-\frac{|k|^{2}}{2}\right] \frac{1}{H_{P, n-1}^{W+}+\alpha_{P, n-1}(\hat{k})|k|-z_{n+1}^{\prime}}
\end{aligned}
$$

This is well defined because for $\sigma_{n} \leq|k| \leq \sigma_{n-1}$, making use of (3.54), (3.55) and the definitions of $\gamma_{P, n+1}, \tilde{\gamma}_{P, n+1}$, we can state

$$
\left|E_{P, n-1}+\Delta c_{P}\right|_{n}^{n-1}+\alpha_{P, n-1}(\hat{k})|k|-\operatorname{Re} z_{n+1}^{\prime}|\geq c| k \mid
$$

for some $c>0$. We first consider the contribution to (4.105) coming from (4.107). It has the form

$$
\begin{array}{r}
-\lambda \int_{\left.\mathcal{A}\right|_{n} ^{n-1}}^{\oplus} \frac{d^{3} k}{\sqrt{2}|k|^{3 / 2}} \frac{k_{i^{\prime}}}{\alpha_{P, n-1}(\hat{k})} \frac{1}{\left[H_{P, n-1}^{W+}\right]_{k}-z_{n+1}}\left(\Gamma_{P, n-1}\right)_{i} \frac{1}{H_{P, n-1}^{W+}+\alpha_{P, n-1}(\hat{k})|k|-z_{n+1}^{\prime}} \phi_{P, n-1} \\
=O\left(|\lambda \| \ln \epsilon|^{1 / 2} \sigma_{n-1}^{-\delta}\right)=O\left(|\lambda|^{1 / 2} \sigma_{n-1}^{-\delta}\right),
\end{array}
$$

where we made use of (4.109), (3.72) and Theorem 4.1. The contribution to (4.105) coming from the term in (4.108) involving $|k|^{2}$ is readily shown to be $O\left(|\lambda|^{1 / 2}\right.$ ) by (4.109), (3.70), and (3.71). Finally, the contribution to (4.105) coming from the term in (4.108) involving $k \cdot \Gamma_{P, n-1}$ has the form

$$
\lambda \int_{\left.\mathcal{A}\right|_{n} ^{n-1}}^{\oplus} \frac{d^{3} k}{\sqrt{2}|k|^{3 / 2}} \frac{k_{i^{\prime}} O\left(|k|^{-1}\right)}{\alpha_{P, n-1}(\hat{k})} \frac{1}{\left[H_{P, n-1}^{W+}\right]_{k}-z_{n+1}}\left(\Gamma_{P, n-1}\right)_{i} \frac{1}{\left[H_{P, n-1}^{W+}\right]_{k}-z_{n+1}^{\prime}}\left(k \cdot \Gamma_{P, n-1}\right) \phi_{P, n-1},
$$

where the $O\left(|k|^{-1}\right)$ insertion comes from (4.109). This expression is $O\left(|\lambda|^{1 / 2} \sigma_{n-1}^{-\delta}|\ln \epsilon|^{1 / 2}\right)$ by (3.71), (3.72) and Theorem 4.1. This concludes the proof of (4.102). Noting that $\mathcal{L}_{n}^{n-1}$ involves one additional power of $k$ compared to $\left.\mathcal{L}\right|_{n} ^{n-1}$, we obtain (4.103) by a completely analogous argument.

\subsection{Contribution to $I^{q}$ with $l \in \mathbb{N}_{0}, l^{\prime} \in \mathbb{N}, l^{\prime \prime}=0$}

In the following lemma we merely recall several facts which appeared in earlier parts of the paper and that will be used repeatedly in the present section. 
Lemma 4.17. Under the assumptions of Theorem 4.3

$$
\begin{aligned}
& \left\|R\left(\Gamma_{P, n-1}\right)_{i}\right\|_{\mathcal{F}_{n}}:=\left\|\frac{1}{H_{P, n-1}^{W+}-z_{n+1}}\left(\Gamma_{P, n-1}\right)_{i}\right\|_{\mathcal{F}_{n}}=O\left(\sigma_{n+1}^{-1}\right), \\
& \|R V\|_{\mathcal{F}_{n}}:=\left\|\left.\frac{1}{H_{P, n-1}^{W+}-z_{n+1}}(-) H_{I}^{W}\right|_{n} ^{n-1}\right\|_{\mathcal{F}_{n}}=O\left(|\lambda|^{1 / 2}\right), \\
& \left\|R\left(\left.\Delta \Gamma_{P}\right|_{n} ^{n-1}\right)_{i}\right\|_{\mathcal{F}_{n}}:=\left\|\frac{1}{H_{P, n-1}^{W+}-z_{n+1}}\left(\left.\Delta \Gamma_{P}\right|_{n} ^{n-1}\right)_{i}\right\|_{\mathcal{F}_{n}}=O\left(\sigma_{n-1}^{-\delta}\right), \\
& \left\|R\left(\Gamma_{P, n-1}\right)_{i^{\prime}} \phi_{P, n-1}\right\|:=\left\|\frac{1}{H_{P, n-1}^{W+}-z_{n+1}}\left(\Gamma_{P, n-1}\right)_{i^{\prime}} \phi_{P, n-1}\right\|=O\left(\sigma_{n-1}^{-\delta}\right)
\end{aligned}
$$

and the same estimates hold for $R$ replaced with $R^{\prime}, R^{\prime \prime}, R^{\prime \prime \prime}$ (cf. definitions (4.16), (4.17)).

Proof. Estimates (4.112) and (4.113) come from Lemma 3.12. As for estimate (4.114), we recall that $\left.\Delta \Gamma_{P}\right|_{n} ^{n-1}:=$ $-\nabla E_{P, n-1}+\nabla E_{P, n}+\left.\mathcal{I}\right|_{n} ^{n-1}+\left.\mathcal{L}\right|_{n} ^{n-1}$ and refer to (4.79) and Lemma 3.12. The bound in (4.115) follows from Theorem 4.1.

The contribution studied in this subsection has the form

$$
\begin{aligned}
I_{\mathbb{N}_{0}, \mathbb{N}, 0}^{q}:= & \left(Q_{P, n-1}^{\perp}\right)^{q} \sum_{l=0}^{\infty}\{R V\}^{l} R\left(\Gamma_{P, n-1}+\left.\Delta \Gamma_{P}\right|_{n} ^{n-1}\right)_{i} \sum_{l^{\prime}=0}^{\infty}\left\{R^{\prime} V\right\}^{l^{\prime}}\left(R^{\prime} V\right) R^{\prime}\left(\Gamma_{P, n-1}+\left.\Delta \Gamma_{P}\right|_{n} ^{n-1}\right)_{i^{\prime}} \phi_{P, n-1} \\
= & \left(Q_{P, n-1}^{\perp}\right)^{q} \sum_{l=0}^{\infty}\{R V\}^{l} R\left(\Gamma_{P, n-1}+\left.\Delta \Gamma_{P}\right|_{n} ^{n-1}\right)_{i} \sum_{l^{\prime}=0}^{\infty}\left\{R^{\prime} V\right\}^{l^{\prime}} Q_{P, n-1}^{\perp}\left(R^{\prime} V\right) R^{\prime}\left(\Gamma_{P, n-1}\right)_{i^{\prime}} \phi_{P, n-1} \\
& +\left(Q_{P, n-1}^{\perp}\right)^{q} \sum_{l=0}^{\infty}\{R V\}^{l} R\left(\Gamma_{P, n-1}+\left.\Delta \Gamma_{P}\right|_{n} ^{n-1}\right)_{i} \sum_{l^{\prime}=0}^{\infty}\left\{R^{\prime} V\right\}^{l^{\prime}} Q_{P, n-1}\left(R^{\prime} V\right) R^{\prime}\left(\Gamma_{P, n-1}\right)_{i^{\prime}} \phi_{P, n-1} \\
& +\left(Q_{P, n-1}^{\perp}\right)^{q} \sum_{l=0}^{\infty}\{R V\}^{l} R\left(\Gamma_{P, n-1}+\left.\Delta \Gamma_{P}\right|_{n} ^{n-1}\right)_{i} \sum_{l^{\prime}=0}^{\infty}\left\{R^{\prime} V\right\}^{l^{\prime}}\left(R^{\prime} V\right) R^{\prime}\left(\left.\Delta \Gamma_{P}\right|_{n} ^{n-1}\right)_{i^{\prime}} \phi_{P, n-1} \\
= & O\left(\sigma_{n-1}^{-2 \delta}\right) .
\end{aligned}
$$

To show this bound, let us first analyse (4.116). By (4.60), $\left(Q_{P, n-1}^{\perp}\right)^{q} \sum_{l=0}^{\infty}\{R V\}^{l}=O(1)$ and $\sum_{l^{\prime}=0}^{\infty}\left\{R^{\prime} V\right\}^{l^{\prime}}=O(1)$, thus we can write

$$
\begin{aligned}
(4.116)= & O(1) R\left(\Gamma_{P, n-1}+\left.\Delta \Gamma_{P}\right|_{n} ^{n-1}\right)_{i} \sum_{l^{\prime}=0}^{\infty}\left\{R^{\prime} V\right\}^{l^{\prime}}\left(b^{*}\left(\left.g_{i^{\prime}}\right|_{n} ^{n-1}\right) \phi_{P, n-1}+O\left(|\lambda|^{1 / 2} \sigma_{n-1}^{1-2 \delta}\right)\right) \\
= & O(1) R\left(\Gamma_{P, n-1}+\left.\Delta \Gamma_{P}\right|_{n} ^{n-1}\right)_{i} \sum_{l^{\prime}=0}^{\infty}\left\{R^{\prime} V\right\}^{l^{\prime}} b^{*}\left(\left.g_{i^{\prime}}\right|_{n} ^{n-1}\right) \phi_{P, n-1}+O\left(\sigma_{n-1}^{-2 \delta}\right) \\
= & O(1) R\left(\Gamma_{P, n-1}+\left.\Delta \Gamma_{P}\right|_{n} ^{n-1}\right)_{i} \sum_{l^{\prime}=0}^{\infty}\left\{R^{\prime} V\right\}^{l^{\prime}} R^{\prime} V b^{*}\left(\left.g_{i^{\prime}}\right|_{n} ^{n-1}\right) \phi_{P, n-1} \\
& +O(1) R\left(\Gamma_{P, n-1}+\left.\Delta \Gamma_{P}\right|_{n} ^{n-1}\right)_{i} b^{*}\left(\left.g_{i^{\prime}}\right|_{n} ^{n-1}\right) \phi_{P, n-1}+O\left(\sigma_{n-1}^{-2 \delta}\right) \\
= & O\left(\sigma_{n-1}^{-2 \delta}\right),
\end{aligned}
$$

where $\left.g_{i^{\prime}}\right|_{n} ^{n-1}$ is a function defined in (4.135), cf. Lemma 4.18. Here, in the first step we used Lemma 4.18 and in the second step Lemma 4.17. The term in (4.120) is estimated using Lemmas 4.19, 4.17. The term in (4.121) 
is estimated using Lemmas 4.20, 4.21. Now we analyse (4.117):

$$
\begin{aligned}
(4.117) & =O(1) R\left(\Gamma_{P, n-1}+\left.\Delta \Gamma_{P}\right|_{n} ^{n-1}\right)_{i} Q_{P, n-1}\left(R^{\prime} V\right) R^{\prime}\left(\Gamma_{P, n-1}\right)_{i^{\prime}} \phi_{P, n-1} \\
& +O(1) R\left(\Gamma_{P, n-1}+\left.\Delta \Gamma_{P}\right|_{n} ^{n-1}\right)_{i} \sum_{l^{\prime}=0}^{\infty}\left\{R^{\prime} V\right\}^{l^{\prime}}\left(R^{\prime} V\right) Q_{P, n-1}\left(R^{\prime} V\right) R^{\prime}\left(\Gamma_{P, n-1}\right)_{i^{\prime}} \phi_{P, n-1} \\
& =O\left(\sigma_{n-1}^{-2 \delta}\right) .
\end{aligned}
$$

Expression (4.122) is estimated using Lemma 4.17. Expression (4.123) is estimated using Lemmas 4.10, 4.17. Finally (4.118) is bounded using Lemma 4.22 and Lemma 4.17.

Lemma 4.18. (Control of (4.116)) Under the assumptions of Theorem 4.3 and the inductive hypothesis

$$
\begin{aligned}
Q_{P, n-1}^{\perp}\left(R^{\prime} V\right) R^{\prime}\left(\Gamma_{P, n-1}\right)_{i^{\prime}} \phi_{P, n-1}: & \left.Q_{P, n-1}^{\perp} \frac{1}{H_{P, n-1}^{W+}-z_{n+1}^{\prime}}(-) H_{I}^{W}\right|_{n} ^{n-1} \frac{1}{H_{P, n-1}^{W+}-z_{n+1}^{\prime}}\left(\Gamma_{P, n-1}\right)_{i^{\prime}} \phi_{P, n-1} \\
& =b^{*}\left(\left.g_{i^{\prime}}\right|_{n} ^{n-1}\right) \phi_{P, n-1}+O\left(|\lambda|^{1 / 2} \sigma_{n-1}^{1-2 \delta}\right),
\end{aligned}
$$

where $b^{*}\left(\left.g_{i^{\prime}}\right|_{n} ^{n-1}\right):=\left.\int d^{3} k g_{i^{\prime}}(k)\right|_{n} ^{n-1} b^{*}(k)$ with $\left.g_{i^{\prime}}\right|_{n} ^{n-1}$ a function with support in $\left.\overline{\mathcal{A}}\right|_{n} ^{n-1}$ which satisfies the pointwise bound

$$
\left|g_{i^{\prime}}(k)\right|_{n}^{n-1}|\leq| \lambda \mid \frac{c}{|k|^{3 / 2} \sigma_{n-1}^{\delta}}
$$

Proof. We recall that $\left.H_{I}^{W}\right|_{n} ^{n-1}:=\left(\left.\mathcal{L}\right|_{n} ^{n-1}+\left.\mathcal{I}\right|_{n} ^{n-1}\right) \cdot \Gamma_{P, n-1}+\Delta\left(\left.H_{I}^{W}\right|_{n} ^{n-1}\right)_{\text {mix }}+\left(\left.H_{I}^{W}\right|_{n} ^{n-1}\right)_{\text {quad }}$ and consider the respective terms. First, by Lemma 3.12 and Theorem 4.1, we obtain that

$$
Q_{P, n-1}^{\perp} \frac{1}{H_{P, n-1}^{W+}-z_{n+1}^{\prime}}\left(\Delta\left(\left.H_{I}^{W}\right|_{n} ^{n-1}\right)_{\mathrm{mix}}+\left(\left.H_{I}^{W}\right|_{n} ^{n-1}\right)_{\mathrm{quad}}\right) \frac{1}{H_{P, n-1}^{W+}-z_{n+1}^{\prime}}\left(\Gamma_{P, n-1}\right)_{i^{\prime}} \phi_{P, n-1}
$$

is $O\left(|\lambda|^{1 / 2} \sigma_{n-1} \sigma_{n-1}^{-\delta}\right)=O\left(|\lambda|^{1 / 2} \sigma_{n-1}^{1-\delta}\right)$. Next, since $\left.|\mathcal{I}|_{n}^{n-1}\left|\leq c_{I}\right| \lambda\right|^{2} \sigma_{n-1}$, we have

$$
Q_{P, n-1}^{\perp} \frac{1}{H_{P, n-1}^{W+}-z_{n+1}^{\prime}}\left(\left.\mathcal{I}\right|_{n} ^{n-1} \cdot \Gamma_{P, n-1}\right) \frac{1}{H_{P, n-1}^{W+}-z_{n+1}^{\prime}}\left(\Gamma_{P, n-1}\right)_{i^{\prime}} \phi_{P, n-1}=O\left(|\lambda|^{2} \sigma_{n-1}^{1-2 \delta}\right),
$$

where we applied the inductive hypothesis of Theorem 4.3. Finally, we observe that in vector (4.128) below we can omit $Q_{P, n-1}^{\perp}$ since this vector is manifestly in its range (cf. Lemma 3.11). Afterwards, we use the direct integral representation and then insert $Q_{P, n-1}+Q_{P, n-1}^{\perp}=1$ on $\mathcal{F}_{n-1}$ inside the direct integral:

$$
\begin{gathered}
Q_{P, n-1}^{\perp} \frac{1}{H_{P, n-1}^{W+}-z_{n+1}^{\prime}}\left(\left.\mathcal{L}\right|_{n} ^{n-1} \cdot \Gamma_{P, n-1}\right) \frac{1}{H_{P, n-1}^{W+}-z_{n+1}^{\prime}}\left(\Gamma_{P, n-1}\right)_{i^{\prime}} \phi_{P, n-1} \\
\simeq-\lambda \int_{\left.\mathcal{A}\right|_{n} ^{n-1}}^{\oplus} \frac{d^{3} k}{\sqrt{2}|k|^{3 / 2}} \frac{k_{i}}{\alpha_{P, n-1}(\hat{k})} \times \\
\quad \times \frac{1}{\left[H_{P, n-1}^{W+}\right]_{k}-z_{n+1}^{\prime}} Q_{P, n-1}^{\perp}\left(\Gamma_{P, n-1}\right)_{i} \frac{1}{H_{P, n-1}^{W+}-z_{n+1}^{\prime}}\left(\Gamma_{P, n-1}\right)_{i^{\prime}} \phi_{P, n-1} \\
-\lambda \int_{\mathcal{A}||_{n}^{n-1}}^{\oplus} \frac{d^{3} k}{\sqrt{2}|k|^{3 / 2}} \frac{k_{i}}{\alpha_{P, n-1}(\hat{k})} \times \\
\quad \times \frac{1}{\left[H_{P, n-1}^{W+}\right]_{k}-z_{n+1}^{\prime}} Q_{P, n-1}\left(\Gamma_{P, n-1}\right)_{i} \frac{1}{H_{P, n-1}^{W+}-z_{n+1}^{\prime}}\left(\Gamma_{P, n-1}\right)_{i^{\prime}} \phi_{P, n-1} .
\end{gathered}
$$


Now using estimate (3.72) we obtain

$$
\|(4.129)\| \leq c|\lambda| \sigma_{n-1}|\ln \epsilon|^{1 / 2}\left\|Q_{P, n-1}^{\perp} \frac{1}{H_{P, n-1}^{W}-z_{n+1}}\left(\Gamma_{P, n-1}\right)_{i} \frac{1}{H_{P, n-1}^{W+}-z_{n+1}}\left(\Gamma_{P, n-1}\right)_{i^{\prime}} \phi_{P, n-1}\right\| \leq c|\lambda|^{1 / 2} \sigma_{n-1}^{1-2 \delta},
$$

where we made use of the induction hypothesis. As for (4.130), with the help of (4.107)-(4.108), (4.109), Lemma 3.14 and Theorem 4.1, we can write

$$
\frac{1}{\left[H_{P, n-1}^{W+}\right]_{k}-z_{n+1}^{\prime}} \phi_{P, n-1}=g_{0}(k) \phi_{P, n-1}+O\left(\sigma_{n-1}^{-\delta}\right),
$$

where $g_{0}$ is a numerical function bounded by $c|k|^{-1}$. We also note that by Theorem 4.1

$$
g_{i, i^{\prime}}:=\frac{1}{\left\|\phi_{P, n-1}\right\|^{2}}\left\langle\phi_{P, n-1},\left(\Gamma_{P, n-1}\right)_{i} \frac{1}{H_{P, n-1}^{W+}-z_{n+1}^{\prime}}\left(\Gamma_{P, n-1}\right)_{i^{\prime}} \phi_{P, n-1}\right\rangle=O\left(\sigma_{n-1}^{-\delta}\right) .
$$

Making use of (4.132), (4.133), (4.131) we get

$$
\text { (4.130) }=b^{*}\left(\left.g_{i^{\prime}}\right|_{n} ^{n-1}\right) \phi_{P, n-1}+O\left(|\lambda||\ln \epsilon|^{1 / 2} \sigma_{n-1}^{1-2 \delta}\right)=b^{*}\left(\left.g_{i^{\prime}}\right|_{n} ^{n-1}\right) \phi_{P, n-1}+O\left(|\lambda|^{1 / 2} \sigma_{n-1}^{1-2 \delta}\right),
$$

where $\left.g_{i^{\prime}}\right|_{n} ^{n-1}$ is the following function from $\left(\left.\mathcal{F}\right|_{n} ^{n-1}\right)^{(1)}$

$$
\left.g_{i^{\prime}}(k)\right|_{n} ^{n-1}:=-\lambda \chi_{\kappa}(k)|k|^{\bar{\alpha}} \frac{1}{\sqrt{2}|k|^{3 / 2}} \frac{k_{i}}{\alpha_{P, n-1}(\hat{k})} g_{0}(k) g_{i, i^{\prime}} .
$$

Clearly, it satisfies the pointwise bound $\left|g_{i^{\prime}}(k)\right|_{n}^{n-1}|\leq| \lambda \mid \frac{c}{|k|^{3 / 2} \sigma_{n-1}^{\delta}}$.

Lemma 4.19. (Control of (4.120)) Under the assumptions of Theorem 4.3

$$
R^{\prime} V b^{*}\left(\left.g_{i^{\prime}}\right|_{n} ^{n-1}\right) \phi_{P, n-1}:=\left.\frac{1}{H_{P, n-1}^{W+}-z_{n+1}^{\prime}}(-) H_{I}^{W}\right|_{n} ^{n-1} b^{*}\left(\left.g_{i^{\prime}}\right|_{n} ^{n-1}\right) \phi_{P, n-1}=O\left(|\lambda| \sigma_{n-1}^{1-2 \delta}\right),
$$

where $\left.g_{i^{\prime}}\right|_{n} ^{n-1}$ was introduced in Lemma 4.18. The same bound holds for $z_{n+1}^{\prime} \in \gamma_{P, n+1}$.

Proof. We recall that $\left.H_{I}^{W}\right|_{n} ^{n-1}:=\left(\left.\mathcal{L}\right|_{n} ^{n-1}+\left.\mathcal{I}\right|_{n} ^{n-1}\right) \cdot \Gamma_{P, n-1}+\Delta\left(\left.H_{I}^{W}\right|_{n} ^{n-1}\right)_{\operatorname{mix}}+\left(\left.H_{I}^{W}\right|_{n} ^{n-1}\right)_{\text {quad }}$ and consider the respective terms. First, by (3.52) and (3.53) in Lemma 3.12 and the fact that $\left\|\left.g_{i^{\prime}}\right|_{n} ^{n-1}\right\|_{2}=O\left(|\lambda|^{1 / 2} \sigma_{n-1}^{-\delta}\right)$, we obtain that

$$
\frac{1}{H_{P, n-1}^{W+}-z_{n+1}^{\prime}}\left(\Delta\left(\left.H_{I}^{W}\right|_{n} ^{n-1}\right)_{\mathrm{mix}}+\left(\left.H_{I}^{W}\right|_{n} ^{n-1}\right)_{\mathrm{quad}}\right) b^{*}\left(\left.g_{i^{\prime}}\right|_{n} ^{n-1}\right) \phi_{P, n-1}=O\left(|\lambda| \sigma_{n-1}^{1-\delta}\right)
$$

Next, making use of $\left.|\mathcal{I}|_{n}^{n-1}\left|\leq c_{I}\right| \lambda\right|^{2} \sigma_{n-1}$, the identity in (4.101) combined with (3.72) in Lemma 3.14, Theorem 4.1 and bound (4.125), we get

$$
\begin{aligned}
& \frac{1}{H_{P, n-1}^{W+}-z_{n+1}^{\prime}}\left(\left.\mathcal{I}\right|_{n} ^{n-1} \cdot \Gamma_{P, n-1}\right) b^{*}\left(\left.g_{i^{\prime}}\right|_{n} ^{n-1}\right) \phi_{P, n-1} \\
& \left.\simeq\left(\left.\mathcal{I}\right|_{n} ^{n-1}\right)_{j} \int_{\left.\mathcal{A}\right|_{n} ^{n-1}}^{\oplus} d^{3} k g^{i^{\prime}}(k)\right|_{n} ^{n-1} \frac{1}{\left[H_{P, n-1}^{W+}\right]_{k}-z_{n+1}^{\prime}}\left(\Gamma_{P, n-1}+k\right)_{j} \phi_{P, n-1}=O\left(|\lambda|^{2} \sigma_{n-1}^{1-2 \delta}\right),
\end{aligned}
$$

where $\left.g_{i^{\prime}}(k)\right|_{n} ^{n-1}=:\left.\chi_{\kappa}(k)|k|^{\bar{\alpha}} g^{i^{\prime}}(k)\right|_{n} ^{n-1}$, as we now indicate the factor $\chi_{\kappa}(k)|k|^{\bar{\alpha}}$ by 'hat' over the integral. Now we look at

$$
\begin{aligned}
\frac{1}{H_{P, n-1}^{W+}-z_{n+1}^{\prime}}\left(\left.\mathcal{L}\right|_{n} ^{n-1} \cdot \Gamma_{P, n-1}\right) b^{*}\left(\left.g_{i^{\prime}}\right|_{n} ^{n-1}\right) \phi_{P, n-1}= & \frac{1}{H_{P, n-1}^{W+}-z_{n+1}^{\prime}}\left(\left(\left.\mathcal{L}\right|_{n} ^{n-1}\right)^{(+)} \cdot \Gamma_{P, n-1}\right) b^{*}\left(\left.g_{i^{\prime}}\right|_{n} ^{n-1}\right) \phi_{P, n-1} \\
& +\frac{1}{H_{P, n-1}^{W+}-z_{n+1}^{\prime}}\left(\left(\left.\mathcal{L}\right|_{n} ^{n-1}\right)^{(-)} \cdot \Gamma_{P, n-1}\right) b^{*}\left(\left.g_{i^{\prime}}\right|_{n} ^{n-1}\right) \phi_{P, n-1} .
\end{aligned}
$$


The direct integral representation, an identity analogous to (4.101) combined with (3.72) of Lemma 3.14, and Theorem 4.1 give

$$
\begin{aligned}
(4.139) & \simeq-\frac{\lambda}{\sqrt{2}} \int_{\left(\left.\mathcal{A l}\right|_{n} ^{n-1}\right)^{\times 2}}^{\oplus} d^{3} k_{1} d^{3} k_{2} \\
& \left(\left.\frac{1}{\sqrt{2}\left|k_{1}\right|^{3 / 2}} \frac{k_{1, j}}{\alpha_{P, n-1}\left(\hat{k}_{1}\right)} g^{i^{\prime}}\left(k_{2}\right)\right|_{n} ^{n-1} \frac{1}{\left[H_{P, n-1}^{W+}\right]_{k_{1}, k_{2}}-z_{n+1}^{\prime}}\left(\Gamma_{P, n-1}+k_{2}\right)_{j} \phi_{P, n-1}+\{1 \leftrightarrow 2\}\right) \\
= & O\left(\lambda^{2}|\ln \epsilon| \sigma_{n-1}^{1-2 \delta}\right)=O\left(|\lambda| \sigma_{n-1}^{1-2 \delta}\right) .
\end{aligned}
$$

As for (4.140), we have by Lemma 3.12, Theorem 4.1 and $|k| \leq \sigma_{n-1}$

$$
\text { (4.140) }=-\left.\lambda \bar{\int}_{\left.\mathcal{A}\right|_{n} ^{n-1}} d^{3} k \frac{1}{\sqrt{2}|k|^{3 / 2}} \frac{k_{j}}{\alpha_{P, n-1}(\hat{k})} g^{i^{\prime}}(k)\right|_{n} ^{n-1}\left(\frac{1}{H_{P, n-1}^{W+}-z_{n+1}^{\prime}}\right)\left(\Gamma_{P, n-1}+k\right)_{j} \phi_{P, n-1},
$$

which is $O\left(|\lambda|^{2} \sigma_{n-1}\left(\sigma_{n-1}^{-\delta}+\epsilon^{-2}\right)|\ln \epsilon| \sigma_{n-1}^{-\delta}\right)=O\left(|\lambda| \sigma_{n-1}^{1-2 \delta}\right)$.

Lemma 4.20. (Control of (4.121)) Under the assumptions of Theorem 4.3

$$
R\left(\Gamma_{P, n-1}\right)_{i} b^{*}\left(\left.g_{i^{\prime}}\right|_{n} ^{n-1}\right) \phi_{P, n-1}:=\frac{1}{H_{P, n-1}^{W+}-z_{n+1}} \Gamma_{P, n-1} b^{*}\left(\left.g_{i^{\prime}}\right|_{n} ^{n-1}\right) \phi_{P, n-1}=O\left(\sigma_{n-1}^{-2 \delta}\right),
$$

where $g_{i^{\prime}}{ }_{n}^{n-1}$ was introduced in Lemma 4.18.

Proof. We write using the direct integral representation

$$
\begin{aligned}
& \frac{1}{H_{P, n-1}^{W+}-z_{n+1}}\left(\Gamma_{P, n-1}\right)_{i} b^{*}\left(g_{i^{\prime}} n_{n}^{n-1}\right) \phi_{P, n-1} \\
& \left.\simeq \int_{\left.\mathcal{A}\right|_{n} ^{n-1}}^{\oplus} d^{3} k g_{i^{\prime}}(k)\right|_{n} ^{n-1} \frac{1}{\left[H_{P, n-1}^{W+}\right]_{k}-z_{n+1}}\left(\Gamma_{P, n-1}+k\right)_{i} \phi_{P, n-1}=O\left(|\lambda||\ln \epsilon|^{\frac{1}{2}} \sigma_{n-1}^{-2 \delta}\right),
\end{aligned}
$$

where we made use of estimate (4.125), the identity in (4.101), (3.72) in Lemma 3.14, and Theorem 4.1.

Lemma 4.21. (Control of (4.121)) Under the assumptions of Theorem 4.3

$$
R\left(\left.\Delta \Gamma_{P}\right|_{n} ^{n-1}\right)_{i} b^{*}\left(\left.g_{i^{\prime}}\right|_{n} ^{n-1}\right) \phi_{P, n-1}:=\frac{1}{H_{P, n-1}^{W+}-z_{n+1}}\left(\left.\Delta \Gamma_{P}\right|_{n} ^{n-1}\right)_{i} b^{*}\left(\left.g_{i^{\prime}}\right|_{n} ^{n-1}\right) \phi_{P, n-1}=O\left(\sigma_{n-1}^{-2 \delta}\right),
$$

where $\left.g_{i^{\prime}}\right|_{n} ^{n-1}$ was introduced in Lemma 4.18.

Proof. We recall that $\left.\Delta \Gamma_{P}\right|_{n} ^{n-1}=\left.X\right|_{n} ^{n-1}+\left.\mathcal{L}\right|_{n} ^{n-1}$, where $\left.X\right|_{n} ^{n-1}:=-\nabla E_{P, n-1}+\nabla E_{P, n}+\left.\mathcal{I}\right|_{n} ^{n-1}$ is a vector in $\mathbb{R}^{3}$ satisfying

$$
\left.|X|_{n}^{n-1}|\leq c| \lambda\right|^{1 / 4} \sigma_{n-1}^{1-\delta}
$$

Since $\left\|\left(H_{P, n-1}^{W+}-z_{n+1}\right)^{-1}\right\|_{\mathcal{F}_{n}}=O\left(\sigma_{n+1}^{-1}\right)$ and $\left\|\left.g_{i^{\prime}}\right|_{n} ^{n-1}\right\|_{2}=O\left(|\lambda|^{1 / 2} \sigma_{n-1}^{-\delta}\right)$, we have

$$
\frac{1}{H_{P, n-1}^{W+}-z_{n+1}}\left(\left.X\right|_{n} ^{n-1}\right)_{i} b^{*}\left(\left.g_{i^{\prime}}\right|_{n} ^{n-1}\right) \phi_{P, n-1}=O\left(|\lambda|^{1 / 2}|\lambda|^{1 / 4} \epsilon^{-2} \sigma_{n-1}^{-2 \delta}\right)=O\left(\sigma_{n-1}^{-2 \delta}\right) .
$$


Next, we consider the contribution with $\left.\mathcal{L}\right|_{n} ^{n-1}$

$$
\begin{aligned}
\frac{1}{H_{P, n-1}^{W+}-z_{n+1}}\left(\left.\mathcal{L}\right|_{n} ^{n-1}\right)_{i} b^{*}\left(\left.g_{i^{\prime}}\right|_{n} ^{n-1}\right) \phi_{P, n-1}=\frac{1}{H_{P, n-1}^{W+}-z_{n+1}}\left(\left.\mathcal{L}\right|_{n} ^{n-1}\right)_{i}^{(+)} b^{*}\left(\left.g_{i^{\prime}}\right|_{n} ^{n-1}\right) \phi_{P, n-1} \\
-\left(\left.\lambda \int_{\left.\mathcal{F}\right|_{n} ^{n-1}} d^{3} k \frac{1}{\sqrt{2}|k|^{3 / 2}} \frac{k_{i}}{\alpha_{P, n-1}(\hat{k})} g_{i^{\prime}}\right|_{n} ^{n-1}(k)\right) \frac{1}{H_{P, n-1}^{W+}-z_{n+1}} \phi_{P, n-1} .
\end{aligned}
$$

To the r.h.s. of (4.148) we apply the direct integral representation

$$
\begin{aligned}
& \text { r.h.s.(4.148) } \simeq-\frac{\lambda}{\sqrt{2}} \int_{\left(\left.\mathcal{A l}\right|_{n} ^{n-1}\right)^{\times 2}}^{\oplus} d^{3} k_{1} d^{3} k_{2} \\
& \qquad\left(\left.\frac{1}{\sqrt{2}\left|k_{1}\right|^{3 / 2}} \frac{k_{1, i}}{\alpha_{P, n-1}\left(\hat{k}_{1}\right)} g^{i^{\prime}}\left(k_{2}\right)\right|_{n} ^{n-1} \frac{1}{\left[H_{P, n-1}^{W+}\right]_{k_{1}, k_{2}}-z_{n+1}} \phi_{P, n-1}+\{1 \leftrightarrow 2\}\right),
\end{aligned}
$$

where $\left.g^{i^{\prime}}\right|_{n} ^{n-1}$ was defined below (4.138). By Lemma 3.14, this gives the bound $O\left(|\lambda|^{2}|\ln \epsilon| \sigma_{n-1}^{-\delta}\right)=O\left(|\lambda| \sigma_{n-1}^{-\delta}\right)$. Finally, the term in (4.149) is clearly $O\left(|\lambda|^{2}|\ln \epsilon| \epsilon^{-2} \sigma_{n-1}^{-\delta}\right)=O\left(|\lambda| \sigma_{n-1}^{-\delta}\right)$.

Lemma 4.22. (Control of (4.118)) Under the assumptions of Theorem 4.3

$$
\begin{aligned}
& \left(R^{\prime} V\right) R^{\prime}\left(\left.\Delta \Gamma_{P}\right|_{n} ^{n-1}\right)_{i^{\prime}} \phi_{P, n-1} \\
& :=\left.\frac{1}{H_{P, n-1}^{W+}-z_{n+1}^{\prime}}(-) H_{I}^{W}\right|_{n} ^{n-1} \frac{1}{H_{P, n-1}^{W+}-z_{n+1}^{\prime}}\left(\left.\Delta \Gamma_{P}\right|_{n} ^{n-1}\right)_{i^{\prime}} \phi_{P, n-1}=O\left(|\lambda|^{1 / 2} \sigma_{n-1}^{1-2 \delta}\right) .
\end{aligned}
$$

Proof. We recall that $\left.H_{I}^{W}\right|_{n} ^{n-1}:=\left(\left.\mathcal{L}\right|_{n} ^{n-1}+\left.\mathcal{I}\right|_{n} ^{n-1}\right) \cdot \Gamma_{P, n-1}+\Delta\left(\left.H_{I}^{W}\right|_{n} ^{n-1}\right)_{\text {mix }}+\left(\left.H_{I}^{W}\right|_{n} ^{n-1}\right)_{\text {quad }}$ with $\left.|\mathcal{I}|_{n}^{n-1}\left|\leq c_{I}\right| \lambda\right|^{2} \sigma_{n-1}$ and $\left.\Delta \Gamma_{P}\right|_{n} ^{n-1}=\left.X\right|_{n} ^{n-1}+\left.\mathcal{L}\right|_{n} ^{n-1}$ with $\left.|X|_{n}^{n-1}|\leq c| \lambda\right|^{1 / 4} \sigma_{n-1}^{1-\delta}$ and analyse the respective terms. First, we have by (3.52) and (3.53) (in Lemma 3.12) and (4.114)

$$
\frac{1}{H_{P, n-1}^{W+}-z_{n+1}^{\prime}}\left(\Delta\left(\left.H_{I}^{W}\right|_{n} ^{n-1}\right)_{\mathrm{mix}}+\left(\left.H_{I}^{W}\right|_{n} ^{n-1}\right)_{\mathrm{quad}}\right) \frac{1}{H_{P, n-1}^{W+}-z_{n+1}^{\prime}}\left(\left.\Delta \Gamma_{P}\right|_{n} ^{n-1}\right)_{i^{\prime}} \phi_{P, n-1}=O\left(|\lambda|^{1 / 2} \sigma_{n-1}^{1-\delta}\right) .
$$

Next, we consider

$$
\begin{aligned}
& \frac{1}{H_{P, n-1}^{W+}-z_{n+1}^{\prime}}\left(\left.\mathcal{I}\right|_{n} ^{n-1} \cdot \Gamma_{P, n-1}\right) \frac{1}{H_{P, n-1}^{W+}-z_{n+1}^{\prime}}\left(\left.X\right|_{n} ^{n-1}\right)_{i^{\prime}} \phi_{P, n-1} \\
& =f\left(z_{n+1}^{\prime}\right)\left(\left.\mathcal{I}\right|_{n} ^{n-1}\right)_{j}\left(\left.X\right|_{n} ^{n-1}\right)_{i^{\prime}} \frac{1}{H_{P, n-1}^{W+}-z_{n+1}^{\prime}}\left(\Gamma_{P, n-1}\right)_{j} \phi_{P, n-1}=O\left(|\lambda|^{2} \sigma_{n-1}^{1-2 \delta}\right),
\end{aligned}
$$

where $f\left(z_{n+1}^{\prime}\right):=\left(E_{P, n-1}+\left.\Delta c_{P}\right|_{n} ^{n-1}-z_{n+1}^{\prime}\right)^{-1}=O\left(\sigma_{n+1}^{-1}\right)$ and we made use of Theorem 4.1. Next, we write, making use of (4.102) in Lemma 4.16

$$
\frac{1}{H_{P, n-1}^{W+}-z_{n+1}^{\prime}}\left(\left.\mathcal{I}\right|_{n} ^{n-1} \cdot \Gamma_{P, n-1}\right) \frac{1}{H_{P, n-1}^{W+}-z_{n+1}^{\prime}}\left(\left.\mathcal{L}\right|_{n} ^{n-1}\right)_{i^{\prime}} \phi_{P, n-1}=O\left(|\lambda|^{5 / 2} \sigma_{n-1}^{1-\delta}\right) .
$$

Now, making use of the direct integral representation, we obtain

$$
\begin{aligned}
& \frac{1}{H_{P, n-1}^{W+}-z_{n+1}^{\prime}}\left(\left.\mathcal{L}\right|_{n} ^{n-1} \cdot \Gamma_{P, n-1}\right) \frac{1}{H_{P, n-1}^{W+}-z_{n+1}^{\prime}}\left(\left.X\right|_{n} ^{n-1}\right)_{i^{\prime}} \phi_{P, n-1} \\
& \simeq f\left(z_{n+1}^{\prime}\right)\left(\left.X\right|_{n} ^{n-1}\right)_{i^{\prime}}(-\lambda) \int_{\left.\mathcal{A}\right|_{n} ^{n-1}}^{\oplus} d^{3} k \frac{1}{\sqrt{2}|k|^{3 / 2}} \frac{k_{j}}{\alpha_{P, n-1}(\hat{k})} \frac{1}{\left[H_{P, n-1}^{W+}\right]_{k}-z_{n+1}^{\prime}}\left(\Gamma_{P, n-1}\right)_{j} \phi_{P, n-1} \\
& =O\left(|\lambda|^{5 / 4} \epsilon^{-2}|\ln \epsilon|^{1 / 2} \sigma_{n-1}^{1-2 \delta}\right)=O\left(|\lambda|^{1 / 2} \sigma_{n-1}^{1-2 \delta}\right),
\end{aligned}
$$


where we made use of (4.101) and (3.72) in Lemma 3.14, and Theorem 4.1. Finally, we obtain from Lemma 4.23

$$
\frac{1}{H_{P, n-1}^{W+}-z_{n+1}^{\prime}}\left(\left.\mathcal{L}\right|_{n} ^{n-1} \cdot \Gamma_{P, n-1}\right) \frac{1}{H_{P, n-1}^{W+}-z_{n+1}^{\prime}}\left(\left.\mathcal{L}\right|_{n} ^{n-1}\right)_{i^{\prime}} \phi_{P, n-1}=O\left(|\lambda| \sigma_{n-1}^{1-\delta}\right) .
$$

This concludes the proof.

Lemma 4.23. Under the assumptions of Theorem 4.3

$$
\begin{aligned}
& \frac{1}{H_{P, n-1}^{W+}-z_{n+1}}\left(\left.\mathcal{L}\right|_{n} ^{n-1} \cdot \Gamma_{P, n-1}\right) \frac{1}{H_{P, n-1}^{W+}-z_{n+1}^{\prime}}\left(\left.\mathcal{L}\right|_{n} ^{n-1}\right)_{i^{\prime}} \phi_{P, n-1}=O\left(|\lambda| \sigma_{n-1}^{1-\delta}\right), \\
& \left.\frac{1}{H_{P, n-1}^{W+}-z_{n+1}}\left(\left.\mathcal{L}\right|_{n} ^{n-1} \cdot \Gamma_{P, n-1}\right) \frac{1}{H_{P, n-1}^{W+}-z_{n+1}^{\prime}} \mathcal{L}\right|_{n} ^{n-1} \phi_{P, n-1}=O\left(|\lambda| \sigma_{n-1}^{2-\delta}\right)
\end{aligned}
$$

for $z_{n+1}, z_{n+1}^{\prime} \in \gamma_{P, n+1} \cup \tilde{\gamma}_{P, n+1}$. Here we denoted

$$
\left(\stackrel{\mathcal{L}}{\left.\right|_{n} ^{n-1}}\right):=\sum_{i=1}^{3}\left[\left(\Gamma_{P, n-1}\right)_{i},\left(\left.\mathcal{L}\right|_{n} ^{n-1}\right)_{i}\right]=\lambda \int_{\sigma_{n}}^{\sigma_{n-1}} d^{3} k \frac{k^{2}\left(b(k)-b^{*}(k)\right)}{\sqrt{2}|k|^{3 / 2} \alpha_{P, n-1}(\hat{k})} .
$$

Proof. We first consider (4.157). We write

$$
\begin{aligned}
\frac{1}{H_{P, n-1}^{W+}-z_{n+1}} & \left(\left.\mathcal{L}\right|_{n} ^{n-1} \cdot \Gamma_{P, n-1}\right) \frac{1}{H_{P, n-1}^{W+}-z_{n+1}^{\prime}}\left(\left.\mathcal{L}\right|_{n} ^{n-1}\right)_{i^{\prime}} \phi_{P, n-1} \\
= & \frac{1}{H_{P, n-1}^{W+}-z_{n+1}}\left(\left(\left.\mathcal{L}\right|_{n} ^{n-1}\right)^{(+)} \cdot \Gamma_{P, n-1}\right) \frac{1}{H_{P, n-1}^{W+}-z_{n+1}^{\prime}}\left(\left.\mathcal{L}\right|_{n} ^{n-1}\right)_{i^{\prime}} \phi_{P, n-1} \\
& +\frac{1}{H_{P, n-1}^{W+}-z_{n+1}}\left(\left(\left.\mathcal{L}\right|_{n} ^{n-1}\right)^{(-)} \cdot \Gamma_{P, n-1}\right) \frac{1}{H_{P, n-1}^{W+}-z_{n+1}^{\prime}}\left(\left.\mathcal{L}\right|_{n} ^{n-1}\right)_{i^{\prime}} \phi_{P, n-1} .
\end{aligned}
$$

Making use of the direct integral representation, we have

$$
\begin{aligned}
(4.161) \simeq & \frac{\lambda^{2}}{\sqrt{2}} \int_{\left(\left.\mathcal{A}\right|_{n} ^{n-1}\right)^{\times 2}}^{\oplus} d^{3} k_{1} d^{3} k_{2}\left(\frac{1}{\sqrt{2}\left|k_{1}\right|^{3 / 2}} \frac{k_{1, j}}{\alpha_{P, n-1}\left(\hat{k}_{1}\right)} \frac{1}{\sqrt{2}\left|k_{2}\right|^{3 / 2}} \frac{k_{2, i^{\prime}}}{\alpha_{P, n-1}\left(\hat{k}_{2}\right)} \times\right. \\
& \left.\times \frac{1}{\left[H_{P, n-1}^{W+}\right]_{k_{1}, k_{2}}-z_{n+1}}\left(\Gamma_{P, n-1}+k_{2}\right)_{j} \frac{1}{\left[H_{P, n-1}^{W+}\right]_{k_{2}}-z_{n+1}^{\prime}} \phi_{P, n-1}+\{1 \leftrightarrow 2\}\right) \\
= & \frac{\lambda^{2}}{\sqrt{2}} \int_{\left(\left.\mathcal{A}\right|_{n} ^{n-1}\right)^{\times 2}}^{\oplus} d^{3} k_{1} d^{3} k_{2}\left(\frac{1}{\sqrt{2}\left|k_{1}\right|^{3 / 2}} \frac{k_{1, j}}{\alpha_{P, n-1}\left(\hat{k}_{1}\right)} \frac{1}{\sqrt{2}\left|k_{2}\right|^{3 / 2}} \frac{k_{2, i^{\prime}}}{\alpha_{P, n-1}\left(\hat{k}_{2}\right)} \times\right. \\
& \left.\times \frac{1}{\left[H_{P, n-1}^{W+}\right]_{k_{1}, k_{2}}-z_{n+1}}\left(\Gamma_{P, n-1}\right)_{j} \frac{1}{\left[H_{P, n-1}^{W+}\right]_{k_{2}}-z_{n+1}^{\prime}} \phi_{P, n-1}+\{1 \leftrightarrow 2\}\right)+O\left(|\lambda|^{2}|\ln \epsilon| \sigma_{n-1}\right),
\end{aligned}
$$

where we made use of (3.70) of Lemma 3.14. Now making use of the resolvent expansion (4.107)-(4.108) we can rewrite a part of the integrand above as follows

$$
\begin{aligned}
& \frac{1}{\left[H_{P, n-1}^{W+}\right]_{k_{1}, k_{2}}-z_{n+1}}\left(\Gamma_{P, n-1}\right)_{i} \frac{1}{\left[H_{P, n-1}^{W+}\right]_{k_{2}}-z_{n+1}^{\prime}} \phi_{P, n-1} \\
& \quad=f_{1}\left(k_{2}\right) \frac{1}{\left[H_{P, n-1}^{W+}\right]_{k_{1}, k_{2}}-z_{n+1}}\left(\Gamma_{P, n-1}\right)_{i} \phi_{P, n-1} \\
& \quad+f_{1}\left(k_{2}\right) \frac{1}{\left[H_{P, n-1}^{W+}\right]_{k_{1}, k_{2}}-z_{n+1}}\left(\Gamma_{P, n-1}\right)_{i} \frac{1}{\left[H_{P, n-1}^{W+}\right]_{k_{2}}-z_{n+1}^{\prime}}\left[-k_{2} \cdot \Gamma_{P, n-1}-\frac{\left|k_{2}\right|^{2}}{2}\right] \phi_{P, n-1} \\
& \quad=O\left(\left|k_{2}\right|^{-1} \sigma_{n-1}^{-\delta}\right),
\end{aligned}
$$


where we set $f_{1}\left(k_{2}\right):=\left(E_{P, n-1}+\left.\Delta c_{P}\right|_{n} ^{n-1}+\alpha_{P, n-1}\left(\hat{k}_{2}\right)\left|k_{2}\right|-z_{n+1}^{\prime}\right)^{-1}$, made use of the fact that $f_{1}\left(k_{2}\right)=O\left(\left|k_{2}\right|^{-1}\right)$ by (4.109) and applied (3.70), (3.71) and (4.101) combined with (3.72) of Lemma 3.14, and Theorem 4.1. Consequently,

$$
(4.161)=O\left(|\lambda|^{2}|\ln \epsilon| \sigma_{n-1}^{1-\delta}\right)=O\left(|\lambda| \sigma_{n-1}^{1-\delta}\right) .
$$

Next, we write

$$
\begin{aligned}
(4.162)= & \lambda^{2} \bar{J}_{\left.\mathcal{A}\right|_{n} ^{n-1}} d^{3} k \frac{1}{2|k|^{3}} \frac{k_{i^{\prime}} k_{j}}{\alpha_{P, n-1}(\hat{k})^{2}}\left(\frac{1}{H_{P, n-1}^{W+}-z_{n+1}}\left(\Gamma_{P, n-1}+k\right)_{j} \frac{1}{\left[H_{P, n-1}^{W+}\right]_{k}-z_{n+1}^{\prime}} \phi_{P, n-1}\right) \\
= & \lambda^{2} \bar{\int}_{\left.\mathcal{A}\right|_{n} ^{n-1}} d^{3} k \frac{1}{2|k|^{3}} \frac{k_{i^{\prime}} k_{j}}{\alpha_{P, n-1}(\hat{k})^{2}}\left(\frac{1}{H_{P, n-1}^{W+}-z_{n+1}}\left(\Gamma_{P, n-1}\right)_{j} \frac{1}{\left[H_{P, n-1}^{W+}\right]_{k}-z_{n+1}^{\prime}} \phi_{P, n-1}\right) \\
& +O\left(|\lambda|^{2}|\ln \epsilon| \epsilon^{-2} \sigma_{n-1}\right),
\end{aligned}
$$

where we made use of (3.70) in Lemma 3.14 and (3.47) in Lemma 3.12. Now we rewrite a part of the integrand in (4.166) using the resolvent expansion, analogously as in (4.164). We have

$$
\begin{aligned}
& \frac{1}{H_{P, n-1}^{W+}-z_{n+1}}\left(\Gamma_{P, n-1}\right)_{i} \frac{1}{\left[H_{P, n-1}^{W+}\right]_{k}-z_{n+1}^{\prime}} \phi_{P, n-1} \\
& \quad=f_{1}(k) \frac{1}{H_{P, n-1}^{W+}-z_{n+1}}\left(\Gamma_{P, n-1}\right)_{i} \phi_{P, n-1} \\
& \quad+f_{1}(k) \frac{1}{H_{P, n-1}^{W+}-z_{n+1}}\left(\Gamma_{P, n-1}\right)_{i} \frac{1}{\left[H_{P, n-1}^{W+}\right]_{k}-z_{n+1}^{\prime}}\left[-k \cdot \Gamma_{P, n-1}-\frac{|k|^{2}}{2}\right] \phi_{P, n-1}=O\left(|k|^{-1} \epsilon^{-2} \sigma_{n-1}^{-\delta}\right),
\end{aligned}
$$

where we used $|k| \leq \sigma_{n-1}$, Lemmas 3.14, 3.12 and Theorem 4.1. Substituting (4.167) to (4.166), we obtain

$$
(4.162)=O\left(|\lambda|^{2}|\ln \epsilon| \epsilon^{-2} \sigma_{n-1}\right)+O\left(|\lambda|^{2}|\ln \epsilon| \epsilon^{-2} \sigma_{n-1}^{1-\delta}\right)=O\left(|\lambda| \sigma_{n-1}^{1-\delta}\right) .
$$

This concludes the proof of (4.157). As for (4.158), we see from (4.159) that $\stackrel{\mathcal{L}}{\left.\right|_{n} ^{n-1}}$ has one power of $k$ more than $\left(\left.\mathcal{L}\right|_{n} ^{n-1}\right)_{i}$. Thus by the same argument as above we obtain the bound by $O\left(|\lambda| \sigma_{n-1}^{2-\delta}\right)$.

\subsection{Contribution to $I^{q}$ with $l \in \mathbb{N}_{0}, l^{\prime} \in \mathbb{N}_{0}, l^{\prime \prime} \in \mathbb{N}$}

The contribution has the form

$$
\begin{aligned}
I_{\mathbb{N}_{0}, \mathbb{N}_{0}, \mathbb{N}}^{q}:=\left(Q_{P, n-1}^{\perp}\right)^{q} \sum_{l=0}^{\infty}\{R V\}^{l} R\left(\Gamma_{P, n-1}+\left.\Delta \Gamma_{P}\right|_{n} ^{n-1}\right)_{i} \sum_{l^{\prime}=0}^{\infty}\left\{R^{\prime} V\right\}^{l^{\prime}} R^{\prime}\left(\Gamma_{P, n-1}+\left.\Delta \Gamma_{P}\right|_{n} ^{n-1}\right)_{i^{\prime}} \times & \times \sum_{l^{\prime \prime}=0}^{\infty} \oint_{\gamma_{P, n+1}} d w_{n+1}\left\{R^{\prime \prime} V\right\}^{l^{\prime \prime}} R^{\prime \prime} V R^{\prime \prime} \phi_{P, n-1} \\
= & O(1) R\left(\Gamma_{P, n-1}+\left.\Delta \Gamma_{P}\right|_{n} ^{n-1}\right)_{i} \sum_{l^{\prime}=0}^{\infty}\left\{R^{\prime} V\right\}^{l^{\prime}} R^{\prime}\left(\Gamma_{P, n-1}+\left.\Delta \Gamma_{P}\right|_{n} ^{n-1}\right)_{i^{\prime}} \oint_{\gamma_{P, n+1}} d w_{n+1} R^{\prime \prime} V R^{\prime \prime} \phi_{P, n-1} \\
+ & O\left(\sigma_{n+1}^{-1}\right) R^{\prime}\left(\Gamma_{P, n-1}+\left.\Delta \Gamma_{P}\right|_{n} ^{n-1}\right)_{i^{\prime}} \sum_{l^{\prime \prime}=0}^{\infty} \oint_{\gamma_{P, n+1}} d w_{n+1}\left(R^{\prime \prime} V\right)^{\ell^{\prime \prime}} R^{\prime \prime} V R^{\prime \prime} V R^{\prime \prime} \phi_{P, n-1},
\end{aligned}
$$

where after splitting $\sum_{l^{\prime \prime}=0}^{\infty}\left\{R^{\prime \prime} V\right\}^{l^{\prime \prime}}=1+\sum_{l^{\prime \prime}=0}^{\infty}\left\{R^{\prime \prime} V\right\}^{l^{\prime \prime}} R^{\prime \prime} V$ we have estimated

$$
\left(Q_{P, n-1}^{\perp}\right)^{q} \sum_{l=0}^{\infty}\{R V\}^{l}=O(1) \quad \text { and } \quad\left(Q_{P, n-1}^{\perp}\right)^{q} \sum_{l=0}^{\infty}\{R V\}^{l} R\left(\Gamma_{P, n-1}+\left.\Delta \Gamma_{P}\right|_{n} ^{n-1}\right)_{i} \sum_{l^{\prime}=0}^{\infty}\left\{R^{\prime} V\right\}^{l^{\prime}}=O\left(\sigma_{n+1}^{-1}\right)
$$


in (4.169) and (4.170), respectively, using Lemma 4.17. To proceed, we set $f\left(w_{n+1}\right):=\left(E_{P, n-1}+\left.\Delta c_{P}\right|_{n} ^{n-1}-\right.$ $\left.w_{n+1}\right)^{-1}=O\left(\sigma_{n+1}^{-1}\right)$ so that $R^{\prime \prime} \phi_{P, n-1}=f\left(w_{n+1}\right) \phi_{P, n-1}$. We first analyse (4.169). Since we have $\left\|R^{\prime}\left(\left.\Delta \Gamma_{P}\right|_{n} ^{n-1}\right)_{i}\right\|_{\mathcal{F}_{n}}=$ $O\left(\sigma_{n-1}^{-\delta}\right),\left\|R^{\prime}\left(\Gamma_{P, n-1}\right)_{i}\right\|_{\mathcal{F}_{n}}=O\left(\sigma_{n+1}^{-1}\right)$ by Lemma 4.17, and Lemma 4.10 gives that $R^{\prime \prime} V \phi_{P, n-1}=O\left(|\lambda|^{1 / 2} \sigma_{n-1}^{1-\delta}\right)$, we obtain

$$
(4.169)=O(1) \oint_{\gamma_{P, n+1}} d w_{n+1} f\left(w_{n+1}\right) R\left(\Gamma_{P, n-1}\right)_{i} \sum_{l^{\prime}=0}^{\infty}\left\{R^{\prime} V\right\}^{l^{\prime}} R^{\prime}\left(\Gamma_{P, n-1}\right)_{i^{\prime}} R^{\prime \prime} V \phi_{P, n-1}+O\left(\sigma_{n-1}^{-2 \delta}\right),
$$

where we also exploited that the length of the integration contour compensates $f\left(w_{n+1}\right)=O\left(\sigma_{n+1}^{-1}\right)$. Using the splitting $1=Q_{P, n-1}^{\perp}+Q_{P, n-1}$, we can write

$$
\begin{aligned}
(4.169)= & O(1) \oint_{\gamma_{P, n+1}} d w_{n+1} f\left(w_{n+1}\right) R\left(\Gamma_{P, n-1}\right)_{i} \sum_{l^{\prime}=0}^{\infty}\left\{R^{\prime} V\right\}^{l^{\prime}} Q_{P, n-1}^{\perp} R^{\prime}\left(\Gamma_{P, n-1}\right)_{i^{\prime}} R^{\prime \prime} V \phi_{P, n-1} \\
& +O(1) \oint_{\gamma_{P, n+1}} d w_{n+1} f\left(w_{n+1}\right) R\left(\Gamma_{P, n-1}\right)_{i} \sum_{l^{\prime}=0}^{\infty}\left\{R^{\prime} V\right\}^{l^{\prime}} R^{\prime} V Q_{P, n-1} R^{\prime}\left(\Gamma_{P, n-1}\right)_{i^{\prime}} R^{\prime \prime} V \phi_{P, n-1} \\
& +O(1) \oint_{\gamma_{P, n+1}} d w_{n+1} f\left(w_{n+1}\right) R\left(\Gamma_{P, n-1}\right)_{i} Q_{P, n-1} R^{\prime}\left(\Gamma_{P, n-1}\right)_{i^{\prime}} R^{\prime \prime} V \phi_{P, n-1}+O\left(\sigma_{n-1}^{-2 \delta}\right) .
\end{aligned}
$$

We analyse (4.173). We have by Lemmas 4.24, 4.19, 4.20

$$
\begin{aligned}
(4.173)= & O(1) \oint_{\gamma_{P, n}} d w_{n+1} f\left(w_{n+1}\right) R\left(\Gamma_{P, n-1}\right)_{i} \sum_{l^{\prime}=0}^{\infty}\left\{R^{\prime} V\right\}^{l^{\prime}} R^{\prime} V b^{*}\left(\left.g_{2, i}\right|_{n} ^{n-1}\right) \phi_{P, n-1} \\
& +O(1) \oint_{\gamma_{P, n}} d w_{n+1} f\left(w_{n+1}\right) R\left(\Gamma_{P, n-1}\right)_{i} b^{*}\left(\left.g_{2, i}\right|_{n} ^{n-1}\right) \phi_{P, n-1}+O\left(\sigma_{n-1}^{-2 \delta}\right) \\
= & O\left(\sigma_{n-1}^{-2 \delta}\right),
\end{aligned}
$$

where $\left.g_{2, i}\right|_{n} ^{n-1}$ is as specified in Lemma 4.33 and analogous to the one introduced in Lemma 4.18 (the only difference being the inessential substitution $\left.\tilde{\gamma}_{P, n+1} \ni z_{n+1}^{\prime} \mapsto w_{n+1} \in \gamma_{P, n+1}\right)$.

Now we consider (4.174) and (4.175). We have by the ingredients stated above (4.172), namely $R^{\prime \prime} V \phi_{P, n-1}=$ $O\left(|\lambda|^{1 / 2} \sigma_{n-1}^{1-\delta}\right)$ and $\left\|R^{\prime}\left(\Gamma_{P, n-1}\right)_{i}\right\|_{\mathcal{F}_{n}}=O\left(\sigma_{n+1}^{-1}\right)$, together with $\left\|R^{\prime}\left(\Gamma_{P, n-1}\right)_{i} \phi_{P, n-1}\right\|=O\left(\sigma_{n-1}^{-\delta}\right)$ of Lemma 4.17

$$
(4.174)=O\left(\sigma_{n-1}^{-2 \delta}\right), \quad \text { and } \quad(4.175)=O\left(\sigma_{n-1}^{-2 \delta}\right) .
$$

Thus altogether

$$
(4.169)=O\left(\sigma_{n-1}^{-2 \delta}\right) \text {. }
$$

Now we analyse (4.170). Using the splitting $1=Q_{P, n-1}^{\perp}+Q_{P, n-1}$ and $\sum_{l^{\prime \prime}=0}^{\infty}\left\{R^{\prime \prime} V\right\}^{l^{\prime \prime}}=1+\sum_{l^{\prime \prime}=0}^{\infty}\left\{R^{\prime \prime} V\right\}^{l^{\prime \prime}} R^{\prime \prime} V$, as well as the estimates $\left\|R^{\prime}\left(\Gamma_{P, n-1}\right)_{i}\right\|_{\mathcal{F}_{n}}=O\left(\sigma_{n+1}^{-1}\right),\left\|R^{\prime}\left(\left.\Delta \Gamma_{P}\right|_{n} ^{n-1}\right)_{i}\right\|_{\mathcal{F}_{n}}=O\left(\sigma_{n-1}^{-\delta}\right)$ of Lemma 4.17, the expression reads

$$
\begin{aligned}
(4.170)= & O\left(\sigma_{n+1}^{-1}\right) R^{\prime}\left(\Gamma_{P, n-1}+\left.\Delta \Gamma_{P}\right|_{n} ^{n-1}\right)_{i^{\prime}} \sum_{l^{\prime \prime}=0}^{\infty} \oint_{\gamma_{P, n+1}} d w_{n+1} f\left(w_{n+1}\right)\left(R^{\prime \prime} V\right)^{\ell^{\prime \prime}} Q_{P, n-1}^{\perp} R^{\prime \prime} V R^{\prime \prime} V \phi_{P, n-1} \\
& +O\left(\sigma_{n+1}^{-1}\right) R^{\prime}\left(\Gamma_{P, n-1}+\left.\Delta \Gamma_{P}\right|_{n} ^{n-1}\right)_{i^{\prime}} \oint_{\gamma_{P, n+1}} d w_{n+1} f\left(w_{n+1}\right) Q_{P, n-1} R^{\prime \prime} V R^{\prime \prime} V \phi_{P, n-1} \\
& +O\left(\sigma_{n+1}^{-2}\right) \sum_{l^{\prime \prime}=0}^{\infty} \oint_{\gamma_{P, n+1}} d w_{n+1} f\left(w_{n+1}\right)\left(R^{\prime \prime} V\right)^{\ell^{\prime \prime}} R^{\prime \prime} V Q_{P, n-1} R^{\prime \prime} V R^{\prime \prime} V \phi_{P, n-1} .
\end{aligned}
$$


Let us consider (4.181). By Lemmas 4.17 and 4.10, we have that

$$
R^{\prime}\left(\Gamma_{P, n-1}+\left.\Delta \Gamma_{P}\right|_{n} ^{n-1}\right)_{i^{\prime}} Q_{P, n-1}=O\left(\sigma_{n-1}^{-\delta}\right), \quad Q_{P, n-1} R^{\prime \prime} V R^{\prime \prime} V \phi_{P, n-1}=O\left(|\lambda| \sigma_{n-1}^{1-\delta}\right),
$$

hence

$$
(4.181)=O\left(\sigma_{n-1}^{-2 \delta}\right)
$$

As for (4.182), recalling from Lemma 4.10 that $R^{\prime \prime} V Q_{P, n-1}=O\left(|\lambda|^{1 / 2} \sigma_{n-1}^{1-\delta}\right)$ and making use of the second formula in (4.183) we get

$$
(4.182)=O\left(\sigma_{n-1}^{-2 \delta}\right)
$$

Now making use of Lemmas 4.25 and 4.17 combined with the splitting $\sum_{l^{\prime \prime}=0}^{\infty}\left\{R^{\prime \prime} V\right\}^{l^{\prime \prime}}=1+\sum_{l^{\prime \prime}=0}^{\infty}\left\{R^{\prime \prime} V\right\}^{l^{\prime \prime}} R^{\prime \prime} V$, we have

$$
\begin{aligned}
(4.180)=\{ & O\left(\sigma_{n+1}^{-1}\right) R^{\prime}\left(\Gamma_{P, n-1}+\left.\Delta \Gamma_{P}\right|_{n} ^{n-1}\right)_{i^{\prime}} \sum_{l^{\prime \prime}=0}^{\infty} \oint_{\gamma_{P, n+1}} d w_{n+1} f\left(w_{n+1}\right)\left(R^{\prime \prime} V\right)^{\ell^{\prime \prime}} \times \\
& \left.\times\left(B^{*}\left(\left.G\right|_{n} ^{n-1}\right) \phi_{P, n-1}+b^{*}\left(\left.\left.\mathcal{I}\right|_{n} ^{n-1} \cdot g\right|_{n} ^{n-1}\right) \phi_{P, n-1}\right)\right\}+O\left(\sigma_{n-1}^{-2 \delta}\right) \\
= & O\left(\sigma_{n+1}^{-1}\right) R^{\prime}\left(\Gamma_{P, n-1}+\left.\Delta \Gamma_{P}\right|_{n} ^{n-1}\right)_{i^{\prime}} B^{*}\left(\left.G\right|_{n} ^{n-1}\right) \phi_{P, n-1} \\
& +O\left(\sigma_{n+1}^{-2}\right) \sum_{l^{\prime \prime}=0}^{\infty} \oint_{\gamma_{P, n+1}} d w_{n+1} f\left(w_{n+1}\right)\left(R^{\prime \prime} V\right)^{\ell^{\prime \prime}} R^{\prime \prime} V B^{*}\left(\left.G\right|_{n} ^{n-1}\right) \phi_{P, n-1} \\
& +O\left(\sigma_{n+1}^{-1}\right) R^{\prime}\left(\Gamma_{P, n-1}+\left.\Delta \Gamma_{P}\right|_{n} ^{n-1}\right)_{i^{\prime}} b^{*}\left(\left.\left.\mathcal{I}\right|_{n} ^{n-1} \cdot g\right|_{n} ^{n-1}\right) \phi_{P, n-1} \\
& +O\left(\sigma_{n+1}^{-2}\right) \sum_{l^{\prime \prime}=0}^{\infty} \oint_{\gamma_{P, n+1}} d w_{n+1} f\left(w_{n+1}\right)\left(R^{\prime \prime} V\right)^{\ell^{\prime \prime}} R^{\prime \prime} V b^{*}\left(\left.\left.\mathcal{I}\right|_{n} ^{n-1} \cdot g\right|_{n} ^{n-1}\right) \phi_{P, n-1} \\
& +O\left(\sigma_{n-1}^{-2 \delta}\right) .
\end{aligned}
$$

We have by Lemma 4.19, formula (4.60) and $\left.|\mathcal{I}|_{n}^{n-1}\left|\leq c_{I}\right| \lambda\right|^{2} \sigma_{n-1}$ that (4.189) $=O\left(\sigma_{n-1}^{-2 \delta}\right)$. Similarly, by Lemmas 4.20, 4.21 and $\left.|\mathcal{I}|_{n}^{n-1}\left|\leq c_{I}\right| \lambda\right|^{2} \sigma_{n-1}$ we have (4.188) $=O\left(\sigma_{n-1}^{-2 \delta}\right)$. Furthermore, by Lemma 4.26 we have $(4.187)=O\left(\sigma_{n-1}^{-2 \delta}\right)$ and by Lemmas 4.28, 4.27, we have (4.186) $=O\left(\sigma_{n-1}^{-2 \delta}\right)$. Thus altogether

$$
(4.180)=O\left(\sigma_{n-1}^{-2 \delta}\right) \text { and }(4.170)=O\left(\sigma_{n-1}^{-2 \delta}\right) .
$$

Thus the proof of Theorem 4.3 is complete, given the auxiliary lemmas listed below.

Lemma 4.24. (Control of (4.173)) Under the assumptions of Theorem 4.3 and the induction hypothesis

$$
\begin{aligned}
Q_{P, n-1}^{\perp} R^{\prime}\left(\Gamma_{P, n-1}\right)_{i^{\prime}} R^{\prime \prime} V \phi_{P, n-1} & :=\left.Q_{P, n-1}^{\perp} \frac{1}{H_{P, n-1}^{W+}-z_{n+1}^{\prime}}\left(\Gamma_{P, n-1}\right)_{i^{\prime}} \frac{1}{H_{P, n-1}^{W+}-w_{n+1}}(-) H_{I}^{W}\right|_{n} ^{n-1} \phi_{P, n-1} \\
& =b^{*}\left(\left.g_{2, i}\right|_{n} ^{n-1}\right) \phi_{P, n-1}+O\left(|\lambda|^{1 / 4} \sigma_{n-1}^{1-2 \delta}\right)
\end{aligned}
$$

where $\left.g_{2, i}\right|_{n} ^{n-1}$ is as specified in Lemma 4.33 and analogous to the one introduced in Lemma 4.18 (the only difference being the inessential substitution $\left.\tilde{\gamma}_{P, n+1} \ni z_{n+1}^{\prime} \mapsto w_{n+1} \in \gamma_{P, n+1}\right)$.

Proof. We recall that $\left.H_{I}^{W}\right|_{n} ^{n-1}:=\left(\left.\mathcal{L}\right|_{n} ^{n-1}+\left.\mathcal{I}\right|_{n} ^{n-1}\right) \cdot \Gamma_{P, n-1}+\Delta\left(\left.H_{I}^{W}\right|_{n} ^{n-1}\right)_{\text {mix }}+\left(\left.H_{I}^{W}\right|_{n} ^{n-1}\right)_{\text {quad }}$ with $\left.|\mathcal{I}|_{n}^{n-1}\left|\leq c_{I}\right| \lambda\right|^{2} \sigma_{n-1}$ and study the respective terms. We have

$$
\left.Q_{P, n-1}^{\perp} \frac{1}{H_{P, n-1}^{W+}-z_{n+1}^{\prime}}\left(\Gamma_{P, n-1}\right)_{i^{\prime}} \frac{1}{H_{P, n-1}^{W+}-w_{n+1}} \mathcal{I}\right|_{n} ^{n-1} \cdot \Gamma_{P, n-1} \phi_{P, n-1}=O\left(|\lambda|^{2} \sigma_{n-1}^{1-2 \delta}\right)
$$


by the induction hypothesis (see Remark 4.5). Furthermore, by Lemma 4.33,

$$
\left.Q_{P, n-1}^{\perp} \frac{1}{H_{P, n-1}^{W+}-z_{n+1}^{\prime}}\left(\Gamma_{P, n-1}\right)_{i^{\prime}} \frac{1}{H_{P, n-1}^{W+}-w_{n+1}} \mathcal{L}\right|_{n} ^{n-1} \cdot \Gamma_{P, n-1} \phi_{P, n-1}=b^{*}\left(\left.g_{2, i}\right|_{n} ^{n-1}\right) \phi_{P, n-1}+O\left(|\lambda|^{1 / 4} \sigma_{n-1}^{1-2 \delta}\right),
$$

where $\left.g_{2, i}\right|_{n} ^{n-1}$ is provided in this lemma. Next, in order to analyse the terms proportional to $\Delta\left(\left.H_{I}^{W}\right|_{n} ^{n-1}\right)_{\operatorname{mix}}$ and $\left(\left.H_{I}^{W}\right|_{n} ^{n-1}\right)_{\text {quad }}$, we recall that

$$
\begin{aligned}
\Delta\left(\left.H_{I}^{W}\right|_{n} ^{n-1}\right)_{\text {mix }} & :=\frac{1}{2} \sum_{i=1}^{3}\left[\left(\Gamma_{P, n-1}\right)_{i},\left(\left.\mathcal{L}\right|_{n} ^{n-1}\right)_{i}\right]=\frac{\lambda}{2} \int_{\left.\mathcal{A}\right|_{n} ^{n-1}} d^{3} k \frac{k^{2}\left(b(k)-b^{*}(k)\right)}{\sqrt{2}|k|^{3 / 2} \alpha_{P, n-1}(\hat{k})}, \\
\left(\left.H_{I}^{W}\right|_{n} ^{n-1}\right)_{\text {quad }} & :=\frac{1}{2}\left(\left.\mathcal{L}\right|_{n} ^{n-1}+\left.\mathcal{I}\right|_{n} ^{n-1}\right)^{2},
\end{aligned}
$$

and note that $\Delta\left(\left.H_{I}^{W}\right|_{n} ^{n-1}\right)_{\text {mix }}$ has the same structure as $\left.\mathcal{L}\right|_{n} ^{n-1}$ (see (3.27)) but is by one power of $k$ more regular. To stress this fact we write $\left.\mathcal{L}\right|_{n} ^{n-1}:=2 \Delta\left(\left.H_{I}^{W}\right|_{n} ^{n-1}\right)_{\text {mix }}$ consistently with the notation in Lemmas B.2, 4.23. We have by Lemma 4.16

$$
\left.Q_{P, n-1}^{\perp} \frac{1}{H_{P, n-1}^{W+}-z_{n+1}^{\prime}}\left(\Gamma_{P, n-1}\right)_{i^{\prime}} \frac{1}{H_{P, n-1}^{W+}-w_{n+1}} \stackrel{\circ}{\mathcal{L}}\right|_{n} ^{n-1} \phi_{P, n-1}=O\left(|\lambda|^{1 / 2} \sigma_{n-1}^{1-\delta}\right) .
$$

Now we study the three terms coming from $\left(\left.H_{I}^{W}\right|_{n} ^{n-1}\right)_{\text {quad }}$. We have by Lemma 4.32

$$
\left.\left.Q_{P, n-1}^{\perp} \frac{1}{H_{P, n-1}^{W+}-z_{n+1}^{\prime}}\left(\Gamma_{P, n-1}\right)_{i^{\prime}} \frac{1}{H_{P, n-1}^{W+}-w_{n+1}} \mathcal{L}\right|_{n} ^{n-1} \cdot \mathcal{L}\right|_{n} ^{n-1} \phi_{P, n-1}=O\left(|\lambda| \sigma_{n-1}^{1-\delta}\right) .
$$

Next, making use of $\left.|\mathcal{I}|_{n}^{n-1}\left|\leq c_{I}\right| \lambda\right|^{2} \sigma_{n-1}$ and Lemma 4.16, we get

$$
\left.\left.Q_{P, n-1}^{\perp} \frac{1}{H_{P, n-1}^{W+}-z_{n+1}^{\prime}}\left(\Gamma_{P, n-1}\right)_{i^{\prime}} \frac{1}{H_{P, n-1}^{W+}-w_{n+1}} \mathcal{I}\right|_{n} ^{n-1} \cdot \mathcal{L}\right|_{n} ^{n-1} \phi_{P, n-1}=O\left(|\lambda|^{5 / 2} \sigma_{n-1}^{1-\delta}\right) .
$$

Finally, by $\left.|\mathcal{I}|_{n}^{n-1}\left|\leq c_{I}\right| \lambda\right|^{2} \sigma_{n-1}$ and Theorem 4.1

$$
Q_{P, n-1}^{\perp} \frac{1}{H_{P, n-1}^{W+}-z_{n+1}^{\prime}}\left(\Gamma_{P, n-1}\right)_{i^{\prime}} \frac{1}{H_{P, n-1}^{W+}-w_{n+1}}\left(\left.\mathcal{I}\right|_{n} ^{n-1}\right)^{2} \phi_{P, n-1}=O\left(|\lambda|^{3} \sigma_{n-1}^{1-\delta}\right) .
$$

This completes the proof.

Lemma 4.25. (Control of (4.180) associated with (4.170)) Under the assumptions of Theorem 4.3 and the induction hypothesis

$$
\begin{aligned}
& Q_{P, n-1}^{\perp} R^{\prime \prime} V R^{\prime \prime} V \phi_{P, n-1} \\
& :=\left.\left.Q_{P, n-1}^{\perp} \frac{1}{H_{P, n-1}^{W+}-w_{n+1}}(-) H_{I}^{W}\right|_{n} ^{n-1} \frac{1}{H_{P, n-1}^{W+}-w_{n+1}}(-) H_{I}^{W}\right|_{n} ^{n-1} \phi_{P, n-1} \\
& =B^{*}\left(\left.G\right|_{n} ^{n-1}\right) \phi_{P, n-1}+b^{*}\left(\left.\left.\mathcal{I}\right|_{n} ^{n-1} \cdot g\right|_{n} ^{n-1}\right) \phi_{P, n-1}+O\left(|\lambda|^{1 / 2} \sigma_{n-1}^{2-2 \delta}\right),
\end{aligned}
$$

where $\left.g_{i}\right|_{n} ^{n-1}$ is a 3-tuple of functions with support in $\left.\overline{\mathcal{A}}\right|_{n} ^{n-1},\left.G\right|_{n} ^{n-1}$ is a function with support in $\left.\overline{\mathcal{A}}\right|_{n} ^{n-1} \times\left.\overline{\mathcal{A}}\right|_{n} ^{n-1}$ and $B^{*}\left(\left.G\right|_{n} ^{n-1}\right):=\left.\int d^{3} k_{1} d^{3} k_{2} G\left(k_{1}, k_{2}\right)\right|_{n} ^{n-1} b^{*}\left(k_{1}\right) b^{*}\left(k_{2}\right)$. They satisfy the pointwise bounds:

$$
\begin{aligned}
& \left.|g(k)|_{n}^{n-1}|\leq c| \lambda|| k\right|^{-3 / 2} \sigma_{n-1}^{-\delta}, \\
& \left.\left|G\left(k_{1}, k_{2}\right)\right|_{n}^{n-1}|\leq c| \lambda\right|^{2}\left|k_{1}\right|^{-3 / 2}\left|k_{2}\right|^{-3 / 2} \sigma_{n-1}^{1-\delta} .
\end{aligned}
$$


Proof. We recall that $\left.H_{I}^{W}\right|_{n} ^{n-1}:=\left(\left.\mathcal{L}\right|_{n} ^{n-1}+\left.\mathcal{I}\right|_{n} ^{n-1}\right) \cdot \Gamma_{P, n-1}+\Delta\left(\left.H_{I}^{W}\right|_{n} ^{n-1}\right)_{\text {mix }}+\left(\left.H_{I}^{W}\right|_{n} ^{n-1}\right)_{\text {quad }}$ with $\left.|\mathcal{I}|_{n}^{n-1}\left|\leq c_{I}\right| \lambda\right|^{2} \sigma_{n-1}$ and study the respective terms.

Step 1: First, we consider

$$
\begin{aligned}
& Q_{P, n-1}^{\perp} \frac{1}{H_{P, n-1}^{W+}-w_{n+1}}\left(\left.\mathcal{L}\right|_{n} ^{n-1}+\left.\mathcal{I}\right|_{n} ^{n-1}\right) \cdot \Gamma_{P, n-1} \frac{1}{H_{P, n-1}^{W+}-w_{n+1}}\left(\left.\mathcal{L}\right|_{n} ^{n-1}+\left.\mathcal{I}\right|_{n} ^{n-1}\right) \cdot \Gamma_{P, n-1} \phi_{P, n-1} \\
&=\left.Q_{P, n-1}^{\perp} \frac{1}{H_{P, n-1}^{W+}-w_{n+1}}\left(\left.\mathcal{L}\right|_{n} ^{n-1}\right)^{(+)} \cdot \Gamma_{P, n-1} \frac{1}{H_{P, n-1}^{W+}-w_{n+1}} \mathcal{L}\right|_{n} ^{n-1} \cdot \Gamma_{P, n-1} \phi_{P, n-1} \\
&+\left.Q_{P, n-1}^{\perp} \frac{1}{H_{P, n-1}^{W+}-w_{n+1}}\left(\left.\mathcal{L}\right|_{n} ^{n-1}\right)^{(-)} \cdot \Gamma_{P, n-1} \frac{1}{H_{P, n-1}^{W+}-w_{n+1}} \mathcal{L}\right|_{n} ^{n-1} \cdot \Gamma_{P, n-1} \phi_{P, n-1} \\
&+\left.\left.Q_{P, n-1}^{\perp} \frac{1}{H_{P, n-1}^{W+}-w_{n+1}} \mathcal{L}\right|_{n} ^{n-1} \cdot \Gamma_{P, n-1} \frac{1}{H_{P, n-1}^{W+}-w_{n+1}} \mathcal{I}\right|_{n} ^{n-1} \cdot \Gamma_{P, n-1} \phi_{P, n-1} \\
&+\left.\left.Q_{P, n-1}^{\perp} \frac{1}{H_{P, n-1}^{W+}-w_{n+1}} \mathcal{I}\right|_{n} ^{n-1} \cdot \Gamma_{P, n-1} \frac{1}{H_{P, n-1}^{W+}-w_{n+1}} \mathcal{L}\right|_{n} ^{n-1} \cdot \Gamma_{P, n-1} \phi_{P, n-1} \\
&+\left.\left.Q_{P, n-1}^{\perp} \frac{1}{H_{P, n-1}^{W+}-w_{n+1}} \mathcal{I}\right|_{n} ^{n-1} \cdot \Gamma_{P, n-1} \frac{1}{H_{P, n-1}^{W+}-w_{n+1}} \mathcal{I}\right|_{n} ^{n-1} \cdot \Gamma_{P, n-1} \phi_{P, n-1} .
\end{aligned}
$$

We note that in (4.205), (4.207), (4.208) we can skip the projection $Q_{P, n-1}^{\perp}$ as the respective vectors are manifestly in its range. Now we estimate the terms above:

$$
\begin{aligned}
(4.205) \simeq & \frac{\lambda^{2}}{\sqrt{2}} \int_{\left(\left.\mathcal{A l}\right|_{n} ^{n-1}\right)^{\times 2}}^{\oplus} d^{3} k_{1} d^{3} k_{2}\left(\frac{1}{\sqrt{2}\left|k_{1}\right|^{3 / 2}} \frac{k_{1, j}}{\alpha_{P, n-1}\left(\hat{k}_{1}\right)} \frac{1}{\sqrt{2}\left|k_{2}\right|^{3 / 2}} \frac{k_{2, j^{\prime}}}{\alpha_{P, n-1}\left(\hat{k}_{2}\right)} \times\right. \\
& \left.\times \frac{1}{\left[H_{P, n-1}^{W+}\right]_{k_{1}, k_{2}}-w_{n+1}}\left(\Gamma_{P, n-1}+k_{2}\right)_{j} \frac{1}{\left[H_{P, n-1}^{W+}\right]_{k_{2}}-w_{n+1}}\left(\Gamma_{P, n-1}\right)_{j^{\prime}} \phi_{P, n-1}+\{1 \leftrightarrow 2\}\right) \\
= & \frac{\lambda^{2}}{\sqrt{2}} \int_{\left(\left.\mathcal{A l}\right|_{n} ^{n-1}\right)^{\times 2}}^{\oplus} d^{3} k_{1} d^{3} k_{2}\left(\frac{1}{\sqrt{2}\left|k_{1}\right|^{3 / 2}} \frac{k_{1, j}}{\alpha_{P, n-1}\left(\hat{k}_{1}\right)} \frac{1}{\sqrt{2}\left|k_{2}\right|^{3 / 2}} \frac{k_{2, j^{\prime}}}{\alpha_{P, n-1}\left(\hat{k}_{2}\right)} \times\right. \\
& \left.\times \frac{1}{\left[H_{P, n-1}^{W+}\right]_{k_{1}, k_{2}}-w_{n+1}} Q_{P, n-1}^{\perp}\left(\Gamma_{P, n-1}\right)_{j} \frac{1}{\left[H_{P, n-1}^{W+}\right]_{k_{2}}-w_{n+1}}\left(\Gamma_{P, n-1}\right)_{j^{\prime}} \phi_{P, n-1}+\{1 \leftrightarrow 2\}\right) \\
& +\frac{\lambda^{2}}{\sqrt{2}} \int_{\left(\left.\mathcal{A}\right|_{n} ^{n-1}\right)^{\times 2}}^{\oplus} d^{3} k_{1} d^{3} k_{2}\left(\frac{1}{\sqrt{2}\left|k_{1}\right|^{3 / 2}} \frac{k_{1, j}}{\alpha_{P, n-1}\left(\hat{k}_{1}\right)} \frac{1}{\sqrt{2}\left|k_{2}\right|^{3 / 2}} \frac{k_{2, j^{\prime}}}{\alpha_{P, n-1}\left(\hat{k}_{2}\right)} \times\right. \\
& \left.\times \frac{1}{\left[H_{P, n-1}^{W+}\right]_{k_{1}, k_{2}}-w_{n+1}} Q_{P, n-1}\left(\Gamma_{P, n-1}\right)_{j} \frac{1}{\left[H_{P, n-1}^{W+}\right]_{k_{2}}-w_{n+1}}\left(\Gamma_{P, n-1}\right)_{j^{\prime}} \phi_{P, n-1}+\{1 \leftrightarrow 2\}\right) \\
+ & O\left(|\lambda| \sigma_{n-1}^{2-\delta}\right),
\end{aligned}
$$

where the error term in (4.213) comes from the part of (4.210) proportional to $k_{2, j}$ and is obtained using Lemma 3.14 and Theorem 4.1. As for the term in (4.211), using (3.72) in Lemma 3.14 we can estimate a part of the integrand as follows

$$
\begin{aligned}
& \left\|\frac{1}{\left[H_{P, n-1}^{W+}\right]_{k_{1}, k_{2}}-w_{n+1}} Q_{P, n-1}^{\perp}\left(\Gamma_{P, n-1}\right)_{j} \frac{1}{\left[H_{P, n-1}^{W+}\right]_{k_{2}}-w_{n+1}}\left(\Gamma_{P, n-1}\right)_{j^{\prime}} \phi_{P, n-1}\right\| \\
& \leq c\left\|Q_{P, n-1}^{\perp} \frac{1}{H_{P, n-1}^{W+}-w_{n+1}}\left(\Gamma_{P, n-1}\right)_{j} \frac{1}{\left[H_{P, n-1}^{W+}\right]_{k_{2}}-w_{n+1}}\left(\Gamma_{P, n-1}\right)_{j^{\prime}} \phi_{P, n-1}\right\|=O\left(\epsilon^{-4} \sigma_{n-1}^{-2 \delta}\right),
\end{aligned}
$$


where in the last step we used Lemma 4.30. Consequently,

$$
(4.211)=O\left(|\lambda|^{2}|\ln \epsilon| \epsilon^{-4} \sigma_{n-1}^{2-2 \delta}\right)=O\left(|\lambda| \sigma_{n-1}^{2-2 \delta}\right) .
$$

Let us now consider the term in (4.212). Using the definition in (3.57), in Lemma 4.29 we show that

$$
\frac{1}{\left[H_{P, n-1}^{W+}\right]_{k_{1}, k_{2}}-w_{n+1}} \phi_{P, n-1}=G_{0}\left(k_{1}, k_{2}\right) \phi_{P, n-1}+O\left(\sigma_{n-1}^{-\delta}\right),
$$

where $G_{0}\left(k_{1}, k_{2}\right)=O\left(\left(\left|k_{1}\right|+\left|k_{2}\right|\right)^{-1}\right)$ is a numerical function, symmetric under the exchange of variables. We also note that by Lemma 3.14, the identity in (4.101), Theorem 4.1, and $\left\|\left(\Gamma_{P, n-1}\right)_{j} \phi_{P, n-1}\right\| \leq c$ (cf. Appendix B) we have

$$
G_{j, j^{\prime}}(k):=\frac{1}{\left\|\phi_{P, n-1}\right\|^{2}}\left\langle\phi_{P, n-1},\left(\Gamma_{P, n-1}\right)_{j} \frac{1}{\left[H_{P, n-1}^{W+}\right]_{k}-w_{n+1}}\left(\Gamma_{P, n-1}\right)_{j^{\prime}} \phi_{P, n-1}\right\rangle=O\left(\sigma_{n-1}^{-\delta}\right) .
$$

Making use of (4.216) and (4.217), we have

$$
(4.212)=B^{*}\left(\left.G\right|_{n} ^{n-1}\right) \phi_{P, n-1}+O\left(|\lambda|^{2}|\ln \epsilon| \sigma_{n-1}^{2-2 \delta}\right)=B^{*}\left(\left.G\right|_{n} ^{n-1}\right) \phi_{P, n-1}+O\left(|\lambda| \sigma_{n-1}^{2-2 \delta}\right),
$$

where $\left.G\right|_{n} ^{n-1}$ is a symmetric function with support in $\left.\overline{\mathcal{A}}\right|_{n} ^{n-1} \times\left.\overline{\mathcal{A}}\right|_{n} ^{n-1}$ given by

$$
\begin{aligned}
\left.G\left(k_{1}, k_{2}\right)\right|_{n} ^{n-1}:= & \lambda^{2} \chi_{\kappa}\left(k_{1}\right) \chi_{\kappa}\left(k_{2}\right)\left|k_{1}\right|^{\bar{\alpha}}\left|k_{2}\right|^{\bar{\alpha}} \frac{k_{1, j}}{\left|k_{1}\right|^{3 / 2} \alpha_{P, n-1}\left(\hat{k}_{1}\right)} \frac{k_{2, j^{\prime}}}{\left|k_{2}\right|^{3 / 2} \alpha_{P, n-1}\left(\hat{k}_{2}\right)} \times \\
& \times G_{0}\left(k_{1}, k_{2}\right) \frac{1}{2}\left(G_{j, j^{\prime}}\left(k_{1}\right)+G_{j, j^{\prime}}\left(k_{2}\right)\right)
\end{aligned}
$$

and $B^{*}\left(\left.G\right|_{n} ^{n-1}\right) \phi_{P, n-1}:=\left.\int d^{3} k_{1} d^{3} k_{2} G\left(k_{1}, k_{2}\right)\right|_{n} ^{n-1} b^{*}\left(k_{1}\right) b^{*}\left(k_{2}\right) \phi_{P, n-1}$. Clearly, we have the pointwise bound

$$
\left.\left|G\left(k_{1}, k_{2}\right)\right|_{n}^{n-1}|\leq| \lambda\right|^{2} \frac{c}{\left|k_{1}\right|^{3 / 2}\left|k_{2}\right|^{3 / 2}} \sigma_{n-1}^{1-\delta},
$$

where we used $\left|k_{1}\right|\left|k_{2}\right| \leq\left|k_{1}\right|^{1 / 2}\left|k_{2}\right|^{1 / 2}\left(\left|k_{1}\right|+\left|k_{2}\right|\right) \leq \sigma_{n-1}\left(\left|k_{1}\right|+\left|k_{2}\right|\right)$.

Now we consider (4.206). It has the form

$$
\begin{aligned}
\text { (4.206) } & =\left.Q_{P, n-1}^{\perp} \frac{1}{H_{P, n-1}^{W+}-w_{n+1}}\left(\left.\mathcal{L}\right|_{n} ^{n-1}\right)^{(-)} \cdot \Gamma_{P, n-1} \frac{1}{H_{P, n-1}^{W+}-w_{n+1}} \mathcal{L}\right|_{n} ^{n-1} \cdot \Gamma_{P, n-1} \phi_{P, n-1} \\
& =\lambda^{2} \int_{\left.\mathcal{A}\right|_{n} ^{n-1}} d^{3} k \frac{1}{2|k|^{3}} \frac{k_{i} k_{i^{\prime}}}{\alpha_{P, n-1}(\hat{k})^{2}} Q_{P, n-1}^{\perp} \frac{1}{H_{P, n-1}^{W+}-w_{n+1}}\left(\Gamma_{P, n-1}+k\right)_{i} \frac{1}{\left[H_{P, n-1}^{W+}\right]_{k}-w_{n+1}}\left(\Gamma_{P, n-1}\right)_{i^{\prime}} \phi_{P, n-1} .
\end{aligned}
$$

The part of (4.206) proportional to $k$ is $O\left(\lambda^{2} \epsilon^{-2}|\ln \epsilon| \sigma_{n-1}^{2-\delta}\right)=O\left(|\lambda| \sigma_{n-1}^{2-\delta}\right)$ due to Lemma 3.14, identity (4.101), Theorem 4.1 and (3.47) in Lemma 3.12. The remaining part of (4.206) is $O\left(|\lambda|^{2}|\ln \epsilon| \epsilon^{-4} \sigma_{n-1}^{2-2 \delta}\right)=O\left(|\lambda| \sigma_{n-1}^{2-2 \delta}\right)$ by Lemma 4.30. Thus altogether

$$
(4.206)=O\left(|\lambda| \sigma_{n-1}^{2-2 \delta}\right) .
$$

Next we estimate (4.207). Proceeding as in the analysis of (4.128) and using $\left.|\mathcal{I}|_{n}^{n-1}\left|\leq c_{I}\right| \lambda\right|^{2} \sigma_{n-1}$ we have

$$
(4.207)=b^{*}\left(\left.\sum_{i^{\prime}=1}^{3}\left(\left.\mathcal{I}\right|_{n} ^{n-1}\right)_{i^{\prime}} g_{1, i^{\prime}}\right|_{n} ^{n-1}\right) \phi_{P, n-1}+O\left(|\lambda|^{5 / 2} \sigma_{n-1}^{2-2 \delta}\right),
$$


where $\left.g_{1, i^{\prime}}\right|_{n} ^{n-1}$ is a 3-tuple of functions with support in $\left.\overline{\mathcal{A}}\right|_{n} ^{n-1}$ analogous to the one introduced in Lemma 4.18 (the only difference being the inessential substitution $\tilde{\gamma}_{P, n+1} \ni z_{n+1}^{\prime} \mapsto w_{n+1} \in \gamma_{P, n+1}$ ) which satisfy the pointwise bound $\left|g_{1, i^{\prime}}(k)\right|_{n}^{n-1}|\leq| \lambda \mid \frac{c}{|k|^{3 / 2} \sigma_{n-1}^{\delta}}$.

Now we consider (4.208). We have by Lemma 4.33 and $\left.|\mathcal{I}|_{n}^{n-1}\left|\leq c_{I}\right| \lambda\right|^{2} \sigma_{n-1}$

$$
(4.208)=b^{*}\left(\left.\sum_{i^{\prime}=1}^{3}\left(\left.\mathcal{I}\right|_{n} ^{n-1}\right)_{i^{\prime}} g_{2, i^{\prime}}\right|_{n} ^{n-1}\right) \phi_{P, n-1}+O\left(|\lambda|^{2} \sigma_{n-1}^{2-2 \delta}\right)
$$

where $\left.g_{2, i^{\prime}}\right|_{n} ^{n-1}$ is a 3-tuple of functions with support in $\left.\overline{\mathcal{A}}\right|_{n} ^{n-1}$ which satisfy the pointwise bound $\left|g_{2, i^{\prime}}(k)\right|_{n}^{n-1} \mid \leq$ $|\lambda| \frac{c}{|k|^{3 / 2} \sigma_{n-1}^{\delta}}$.

Since it is readily seen that (4.209) $=O\left(|\lambda|^{4} \sigma_{n-1}^{2-2 \delta}\right)$ using $\left.|\mathcal{I}|_{n}^{n-1}\left|\leq c_{I}\right| \lambda\right|^{2} \sigma_{n-1}$ and the inductive hypothesis, our analysis of (4.204) is complete. Summing up, we have shown that

$$
(4.204)=\left\{B^{*}\left(\left.G\right|_{n} ^{n-1}\right)+b^{*}\left(\left.\sum_{i^{\prime}=1}^{3}\left(\left.\mathcal{I}\right|_{n} ^{n-1}\right)_{i^{\prime}} g_{2, i^{\prime}}\right|_{n} ^{n-1}\right)+b^{*}\left(\left.\sum_{i=1}^{3}\left(\left.\mathcal{I}\right|_{n} ^{n-1}\right)_{i} g_{1, i}\right|_{n} ^{n-1}\right)\right\} \phi_{P, n-1}+O\left(|\lambda| \sigma_{n-1}^{2-2 \delta}\right),
$$

where $\left.\left|G\left(k_{1}, k_{2}\right)\right|_{n}^{n-1}|\leq c| \lambda\right|^{2}\left|k_{1}\right|^{-3 / 2}\left|k_{2}\right|^{-3 / 2} \sigma_{n-1}^{1-\delta}$ and $\left\{\left.\sum_{i=1}^{3}\left|g_{\#, i}(k)\right|_{n}^{n-1}\right|^{2}\right\}^{\frac{1}{2}} \leq c|\lambda||k|^{-3 / 2} \sigma_{n-1}^{-\delta}$ with \# = 1,2.

Step 2: Now we consider the following contribution to the expression from the statement of the lemma

$$
\begin{aligned}
& Q_{P, n-1}^{\perp} \frac{1}{H_{P, n-1}^{W+}-w_{n+1}}\left(\Delta\left(\left.H_{I}^{W}\right|_{n} ^{n-1}\right)_{\text {mix }}+\left(\left.H_{I}^{W}\right|_{n} ^{n-1}\right)_{\text {quad }}\right) \frac{1}{H_{P, n-1}^{W+}-w_{n+1}}\left(\left.\mathcal{L}\right|_{n} ^{n-1}+\left.\mathcal{I}\right|_{n} ^{n-1}\right) \cdot \Gamma_{P, n-1} \phi_{P, n-1} \\
&= O\left(|\lambda|^{1 / 2} \sigma_{n-1}\right) \frac{1}{H_{P, n-1}^{W+}-w_{n+1}}\left(\left.\mathcal{L}\right|_{n} ^{n-1} \cdot \Gamma_{P, n-1}\right) \phi_{P, n-1} \\
&+O\left(|\lambda|^{1 / 2} \sigma_{n-1}\right) \frac{1}{H_{P, n-1}^{W+}-w_{n+1}}\left(\left.\mathcal{I}\right|_{n} ^{n-1} \cdot \Gamma_{P, n-1}\right) \phi_{P, n-1}
\end{aligned}
$$

where we made use of (3.52) and (3.53) in Lemma 3.12. Clearly (4.228) $=O\left(|\lambda|^{5 / 2} \sigma_{n-1}^{2-\delta}\right.$ ) due to $|\mathcal{I}|_{n}^{n-1} \mid \leq$ $c_{I}|\lambda|^{2} \sigma_{n-1}$ and Theorem 4.1. Furthermore, we have by Lemma 4.15 that (4.227) $=O\left(|\lambda| \sigma_{n-1}^{2-\delta}\right)$. Thus altogether

$$
(4.226)=O\left(|\lambda| \sigma_{n-1}^{2-\delta}\right) \text {. }
$$

Step 3: Next we consider the following contribution to the expression from the statement of the lemma

$$
\begin{aligned}
& Q_{P, n-1}^{\perp} \frac{1}{H_{P, n-1}^{W+}-w_{n+1}}\left(\left.\mathcal{L}\right|_{n} ^{n-1}+\left.\mathcal{L}\right|_{n} ^{n-1}\right) \cdot \Gamma_{P, n-1} \times \\
& \quad \times \frac{1}{H_{P, n-1}^{W+}-w_{n+1}}\left(\Delta\left(\left.H_{I}^{W}\right|_{n} ^{n-1}\right)_{\mathrm{mix}}+\left(\left.H_{I}^{W}\right|_{n} ^{n-1}\right)_{\mathrm{quad}}\right) \phi_{P, n-1} \\
& =\left.Q_{P, n-1}^{\perp} \frac{1}{H_{P, n-1}^{W+}-w_{n+1}} \mathcal{I}\right|_{n} ^{n-1} \cdot \Gamma_{P, n-1} \frac{1}{H_{P, n-1}^{W+}-w_{n+1}} \Delta\left(\left.H_{I}^{W}\right|_{n} ^{n-1}\right)_{\operatorname{mix}} \phi_{P, n-1} \\
& \quad+\left.Q_{P, n-1}^{\perp} \frac{1}{H_{P, n-1}^{W+}-w_{n+1}} \mathcal{L}\right|_{n} ^{n-1} \cdot \Gamma_{P, n-1} \frac{1}{H_{P, n-1}^{W+}-w_{n+1}} \Delta\left(\left.H_{I}^{W}\right|_{n} ^{n-1}\right)_{\operatorname{mix}} \phi_{P, n-1} \\
& \quad+\left.Q_{P, n-1}^{\perp} \frac{1}{H_{P, n-1}^{W+}-w_{n+1}} \mathcal{I}\right|_{n} ^{n-1} \cdot \Gamma_{P, n-1} \frac{1}{H_{P, n-1}^{W+}-w_{n+1}}\left(\left.H_{I}^{W}\right|_{n} ^{n-1}\right)_{\mathrm{quad}} \phi_{P, n-1} \\
& \quad+\left.Q_{P, n-1}^{\perp} \frac{1}{H_{P, n-1}^{W+}-w_{n+1}} \mathcal{L}\right|_{n} ^{n-1} \cdot \Gamma_{P, n-1} \frac{1}{H_{P, n-1}^{W+}-w_{n+1}}\left(\left.H_{I}^{W}\right|_{n} ^{n-1}\right)_{\mathrm{quad}} \phi_{P, n-1} .
\end{aligned}
$$


To analyse these expressions, norm-bounds involving $\Delta\left(\left.H_{I}^{W}\right|_{n} ^{n-1}\right)_{\operatorname{mix}},\left(\left.H_{I}^{W}\right|_{n} ^{n-1}\right)_{\text {quad }}$ from Lemma 3.12 are not sufficient and we have to recall the definitions:

$$
\begin{aligned}
\Delta\left(\left.H_{I}^{W}\right|_{n} ^{n-1}\right)_{\text {mix }} & :=\sum_{i=1}^{3} \frac{1}{2}\left[\left(\Gamma_{P, n-1}\right)_{i},\left(\left.\mathcal{L}\right|_{n} ^{n-1}\right)_{i}\right]=\frac{\lambda}{2} \int_{\left.\mathcal{A}\right|_{n} ^{n-1}} d^{3} k \frac{k^{2}\left(b(k)-b^{*}(k)\right)}{\sqrt{2}|k|^{3 / 2} \alpha_{P, n-1}(\hat{k})}, \\
\left(\left.H_{I}^{W}\right|_{n} ^{n-1}\right)_{\text {quad }} & :=\frac{1}{2}\left(\left.\mathcal{L}\right|_{n} ^{n-1}+\left.\mathcal{I}\right|_{n} ^{n-1}\right)^{2} .
\end{aligned}
$$

We note that $\Delta\left(\left.H_{I}^{W}\right|_{n} ^{n-1}\right)_{\text {mix }}$ has the same structure as $\left.\mathcal{L}\right|_{n} ^{n-1}$ but is by one power of $k$ more regular. To stress this fact we write $\left.\mathcal{L}\right|_{n} ^{n-1}:=2 \Delta\left(\left.H_{I}^{W}\right|_{n} ^{n-1}\right)_{\text {mix }}$ consistently with the notation in Lemmas B.2, 4.16, 4.23.

Thus we immediately get by Lemma 4.16 and $\left.|\mathcal{I}|_{n}^{n-1}\left|\leq c_{I}\right| \lambda\right|^{2} \sigma_{n-1}$, that

$$
(4.231)=O\left(|\lambda|^{5 / 2} \sigma_{n-1}^{2-\delta}\right) .
$$

Similarly, by Lemma 4.23 we obtain

$$
(4.232)=O\left(|\lambda| \sigma_{n-1}^{2-\delta}\right) .
$$

Now proceeding to (4.233), we write

$$
\begin{aligned}
2 \times(4.233)= & \left.Q_{P, n-1}^{\perp} \frac{1}{H_{P, n-1}^{W+}-w_{n+1}} \mathcal{I}\right|_{n} ^{n-1} \cdot \Gamma_{P, n-1} \frac{1}{H_{P, n-1}^{W+}-w_{n+1}}\left(\left.\mathcal{I}\right|_{n} ^{n-1}\right)^{2} \phi_{P, n-1} \\
& +\left.Q_{P, n-1}^{\perp} \frac{1}{H_{P, n-1}^{W+}-w_{n+1}} \mathcal{I}\right|_{n} ^{n-1} \cdot \Gamma_{P, n-1} \frac{1}{H_{P, n-1}^{W+}-w_{n+1}}\left(\left.\left.2 \mathcal{I}\right|_{n} ^{n-1} \cdot \mathcal{L}\right|_{n} ^{n-1}\right) \phi_{P, n-1} \\
& +\left.Q_{P, n-1}^{\perp} \frac{1}{H_{P, n-1}^{W+}-w_{n+1}} \mathcal{I}\right|_{n} ^{n-1} \cdot \Gamma_{P, n-1} \frac{1}{H_{P, n-1}^{W+}-w_{n+1}}\left(\left.\mathcal{L}\right|_{n} ^{n-1}\right) \cdot\left(\left.\mathcal{L}\right|_{n} ^{n-1}\right) \phi_{P, n-1} .
\end{aligned}
$$

Using $\left.|\mathcal{I}|_{n}^{n-1}\left|\leq c_{I}\right| \lambda\right|^{2} \sigma_{n-1}$, the fact that $\left(H_{P, n-1}^{W+}-w_{n+1}\right)^{-1} \phi_{P, n-1}=O\left(\sigma_{n+1}^{-1}\right)$ and Theorem 4.1, we have

$$
(4.239)=O\left(|\lambda|^{6} \epsilon^{-2} \sigma_{n-1}^{2-\delta}\right)=O\left(|\lambda|^{5} \sigma_{n-1}^{2-\delta}\right) \text {. }
$$

Next, by Lemma 4.16 and $\left.|\mathcal{I}|_{n}^{n-1}\left|\leq c_{I}\right| \lambda\right|^{2} \sigma_{n-1}$

$$
(4.240)=O\left(|\lambda|^{9 / 2} \sigma_{n-1}^{2-\delta}\right) \text {. }
$$

Now we consider (4.241). We have by Lemma 4.32 and $\left.|\mathcal{I}|_{n}^{n-1}\left|\leq c_{I}\right| \lambda\right|^{2} \sigma_{n-1}$

$$
(4.241)=O\left(|\lambda|^{3} \sigma_{n-1}^{2-\delta}\right) .
$$

Thus, altogether, we have

$$
(4.233)=O\left(|\lambda|^{3} \sigma_{n-1}^{2-\delta}\right) .
$$

Finally, we consider (4.234). We have

$$
\begin{aligned}
2 \times(4.234)= & Q_{P, n-1}^{\perp} \frac{1}{H_{P, n-1}^{W+}-w_{n+1}}\left(\left.\mathcal{L}\right|_{n} ^{n-1}\right)^{(+)} \cdot \Gamma_{P, n-1} \frac{1}{H_{P, n-1}^{W+}-w_{n+1}}\left(\left.\mathcal{I}\right|_{n} ^{n-1}\right)^{2} \phi_{P, n-1} \\
& +\left.Q_{P, n-1}^{\perp} \frac{1}{H_{P, n-1}^{W+}-w_{n+1}} \mathcal{L}\right|_{n} ^{n-1} \cdot \Gamma_{P, n-1} \frac{1}{H_{P, n-1}^{W+}-w_{n+1}}\left(\left.2 \mathcal{I}\right|_{n} ^{n-1} \cdot\left(\left.\mathcal{L}\right|_{n} ^{n-1}\right)^{(+)}\right) \phi_{P, n-1} \\
& +Q_{P, n-1}^{\perp} \frac{1}{H_{P, n-1}^{W+}-w_{n+1}}\left(\left.\mathcal{L}\right|_{n} ^{n-1}\right)^{(+)} \cdot \Gamma_{P, n-1} \frac{1}{H_{P, n-1}^{W+}-w_{n+1}}\left(\left(\left.\mathcal{L}\right|_{n} ^{n-1}\right)^{(+)} \cdot\left(\left.\mathcal{L}\right|_{n} ^{n-1}\right)^{(+)}\right) \phi_{P, n-1} \\
& +Q_{P, n-1}^{\perp} \frac{1}{H_{P, n-1}^{W+}-w_{n+1}}\left(\left.\mathcal{L}\right|_{n} ^{n-1}\right)^{(-)} \cdot \Gamma_{P, n-1} \frac{1}{H_{P, n-1}^{W+}-w_{n+1}}\left(\left(\left.\mathcal{L}\right|_{n} ^{n-1}\right)^{(+)} \cdot\left(\left.\mathcal{L}\right|_{n} ^{n-1}\right)^{(+)}\right) \phi_{P, n-1} \\
& +Q_{P, n-1}^{\perp} \frac{1}{H_{P, n-1}^{W+}-w_{n+1}}\left(\left.\mathcal{L}\right|_{n} ^{n-1}\right)^{(+)} \cdot \Gamma_{P, n-1} \frac{1}{H_{P, n-1}^{W+}-w_{n+1}}\left(\left(\left.\mathcal{L}\right|_{n} ^{n-1}\right)^{(-)} \cdot\left(\left.\mathcal{L}\right|_{n} ^{n-1}\right)^{(+)}\right) \phi_{P, n-1} .
\end{aligned}
$$


Making use of $\left.|\mathcal{I}|_{n}^{n-1}\left|\leq c_{I}\right| \lambda\right|^{2} \sigma_{n-1}$ and applying Lemma 4.15, we easily see

$$
(4.246)=O\left(\lambda^{4} \sigma_{n-1}^{2-\delta}\right) \text {. }
$$

Next, applying Lemma 4.23 and using $\left.|\mathcal{I}|_{n}^{n-1}\left|\leq c_{I}\right| \lambda\right|^{2} \sigma_{n-1}$, we have

$$
(4.247)=O\left(|\lambda|^{3} \sigma_{n-1}^{2-\delta}\right) \text {. }
$$

Now we consider (4.248) where we can omit $Q_{P, n-1}^{\perp}$. We can write

$$
\begin{aligned}
(4.248) \simeq & \frac{(-\lambda)^{3}}{\sqrt{3 !}} \int_{\left(\left.\mathcal{A}\right|_{n} ^{n-1}\right)^{\times 3}}^{\oplus} d^{3} k_{1} d^{3} k_{2} d^{3} k_{3} \\
& \left\{\frac{1}{\sqrt{2}\left|k_{1}\right|^{3 / 2}} \frac{k_{1, j}}{\alpha_{P, n-1}\left(\hat{k}_{1}\right)} \frac{1}{\sqrt{2}\left|k_{2}\right|^{3 / 2}} \frac{k_{2, j}}{\alpha_{P, n-1}\left(\hat{k}_{2}\right)} \frac{1}{\sqrt{2}\left|k_{3}\right|^{3 / 2}} \frac{k_{3, j^{\prime}}}{\alpha_{P, n-1}\left(\hat{k}_{3}\right)} \times\right. \\
& \left.\times \frac{1}{\left[H_{P, n-1}^{W+}\right]_{k_{1}, k_{2}, k_{3}}-w_{n+1}}\left(\Gamma_{P, n-1}+k_{1}+k_{2}\right)_{j^{\prime}} \frac{1}{\left[H_{P, n-1}^{W+}\right]_{k_{1}, k_{2}}-w_{n+1}} \phi_{P, n-1}+\{\text { perm }\}\right\},
\end{aligned}
$$

where $\{$ perm $\}$ denotes all the remaining permutations of the variables $k_{1}, k_{2}, k_{3}$ and summation over $j, j^{\prime}$ is understood. The part of (4.253) which is proportional to $k_{1}+k_{2}$ is $O\left(|\lambda|^{3}|\ln \epsilon|^{3 / 2} \sigma_{n-1}^{2}\right.$ ) by (3.70), (3.71) in Lemma 3.14. Furthermore, we have by Lemmas 4.29, 3.14 and Theorem 4.1

$$
\begin{aligned}
& \frac{1}{\left[H_{P, n-1}^{W+}\right]_{k_{1}, k_{2}, k_{3}}-w_{n+1}}\left(\Gamma_{P, n-1}\right)_{j^{\prime}} \frac{1}{\left[H_{P, n-1}^{W+}\right]_{k_{1}, k_{2}}-w_{n+1}} \phi_{P, n-1} \\
& \quad=G_{0}\left(k_{1}, k_{2}\right) \frac{1}{\left[H_{P, n-1}^{W+}\right]_{k_{1}, k_{2}, k_{3}}-w_{n+1}}\left(\Gamma_{P, n-1}\right)_{j^{\prime}} \phi_{P, n-1}+\frac{1}{\left[H_{P, n-1}^{W+}\right]_{k_{1}, k_{2}, k_{3}}-w_{n+1}}\left(\Gamma_{P, n-1}\right)_{j^{\prime}} O\left(\sigma_{n-1}^{-\delta}\right) \\
& \quad=O\left(\left|k_{1}\right|^{-1} \sigma_{n-1}^{-\delta}\right) .
\end{aligned}
$$

By substituting (4.254) to (4.253), we have

$$
(4.248)=O\left(|\lambda|^{2} \sigma_{n-1}^{2-\delta}\right) .
$$

Next, we estimate (4.249). We have

$$
\begin{aligned}
(4.249) \simeq & 2(-\lambda)^{3} \int_{\left.\mathcal{F}\right|_{n} ^{n-1}}^{\oplus} d^{3} k^{\prime} \frac{1}{\sqrt{2}\left|k^{\prime}\right|^{3 / 2}} \frac{k_{j^{\prime}}^{\prime}}{\alpha_{P, n-1}\left(\hat{k}^{\prime}\right)} \int_{\left.\mathcal{P}\right|_{n} ^{n-1}} d^{3} k \frac{1}{2|k|^{3}} \frac{k_{j^{\prime}} k_{j}}{\alpha_{P, n-1}(\hat{k})^{2}} \times \\
& \times \frac{1}{\left[H_{P, n-1}^{W+}\right]_{k^{\prime}}-w_{n+1}}\left(\Gamma_{P, n-1}+k+k^{\prime}\right)_{j} \frac{1}{\left[H_{P, n-1}^{W+}\right]_{k, k^{\prime}}-w_{n+1}} \phi_{P, n-1} .
\end{aligned}
$$

We note that by Lemma 3.14 the term proportional to $k+k^{\prime}$ in this expression is $O\left(|\lambda|^{3}|\ln \epsilon|^{3 / 2} \sigma_{n-1}^{2}\right)$. Furthermore, we have by Lemmas 4.29, 3.14 and Theorem 4.1,

$$
\begin{aligned}
& \frac{1}{\left[H_{P, n-1}^{W+}\right]_{k^{\prime}}-w_{n+1}}\left(\Gamma_{P, n-1}\right)_{j^{\prime}} \frac{1}{\left[H_{P, n-1}^{W+}\right]_{k, k^{\prime}}-w_{n+1}} \phi_{P, n-1} \\
& =G_{0}\left(k, k^{\prime}\right) \frac{1}{\left[H_{P, n-1}^{W+}\right]_{k^{\prime}}-w_{n+1}}\left(\Gamma_{P, n-1}\right)_{j^{\prime}} \phi_{P, n-1}+\frac{1}{\left[H_{P, n-1}^{W+}\right]_{k^{\prime}}-w_{n+1}}\left(\Gamma_{P, n-1}\right)_{j^{\prime}} O\left(\sigma_{n-1}^{-\delta}\right)=O\left(\left|k^{\prime}\right|^{-1} \sigma_{n-1}^{-\delta}\right) .
\end{aligned}
$$

By substituting (4.257) to (4.256), we have altogether

$$
(4.249)=O\left(|\lambda|^{2} \sigma_{n-1}^{2-\delta}\right) .
$$


Finally, we consider (4.250). Making use of (4.311) and Lemma 4.15, we have

$$
(4.250)=O\left(|\lambda|^{2}|\ln \epsilon| \epsilon^{-2} \sigma_{n-1}\right) \frac{1}{H_{P, n-1}^{W+}-w_{n+1}}\left(\left.\mathcal{L}\right|_{n} ^{n-1}\right)^{(+)} \cdot \Gamma_{P, n-1} \phi_{P, n-1}=O\left(|\lambda| \sigma_{n-1}^{2-\delta}\right) .
$$

Thus altogether

$$
(4.234)=O\left(|\lambda| \sigma_{n-1}^{2-\delta}\right)
$$

Summing up,

$$
(4.230)=O\left(|\lambda| \sigma_{n-1}^{2-\delta}\right) .
$$

Step 4: Finally, we consider the following contribution to the expression from the statement of the lemma

$$
\begin{aligned}
& Q_{P, n-1}^{\perp} \frac{1}{H_{P, n-1}^{W+}-w_{n+1}}\left(\Delta\left(\left.H_{I}^{W}\right|_{n} ^{n-1}\right)_{\mathrm{mix}}+\left(\left.H_{I}^{W}\right|_{n} ^{n-1}\right)_{\text {quad }}\right) \times \\
& \quad \times \frac{1}{H_{P, n-1}^{W+}-w_{n+1}}\left(\Delta\left(\left.H_{I}^{W}\right|_{n} ^{n-1}\right)_{\mathrm{mix}}+\left(\left.H_{I}^{W}\right|_{n} ^{n-1}\right)_{\mathrm{quad}}\right) \phi_{P, n-1}=O\left(|\lambda|^{1 / 2} \sigma_{n-1}^{2}\right),
\end{aligned}
$$

where we made use of (3.52) and (3.53) in Lemma 3.12. This concludes the proof.

Lemma 4.26. (Control of (4.187) associated with (4.180)) Under the assumptions of Theorem 4.3

$$
R^{\prime \prime} V B^{*}\left(\left.G\right|_{n} ^{n-1}\right) \phi_{P, n-1}:=\left.\frac{1}{H_{P, n-1}^{W+}-w_{n+1}}(-) H_{I}^{W}\right|_{n} ^{n-1} B^{*}\left(\left.G\right|_{n} ^{n-1}\right) \phi_{P, n-1}=O\left(|\lambda|^{3 / 2} \sigma_{n-1}^{2-2 \delta}\right),
$$

where $B^{*}\left(\left.G\right|_{n} ^{n-1}\right)$ appeared in Lemma 4.25 .

Proof. We recall that $\left.H_{I}^{W}\right|_{n} ^{n-1}:=\left(\left.\mathcal{L}\right|_{n} ^{n-1}+\left.\mathcal{I}\right|_{n} ^{n-1}\right) \cdot \Gamma_{P, n-1}+\Delta\left(\left.H_{I}^{W}\right|_{n} ^{n-1}\right)_{\operatorname{mix}}+\left(\left.H_{I}^{W}\right|_{n} ^{n-1}\right)_{\text {quad }}$ and consider the respective terms. First, by (3.52)-(3.53) in Lemma 3.12 combined with the fact that $\left\|\left.G\right|_{n} ^{n-1}\right\|_{2}=O\left(|\lambda| \sigma_{n-1}^{1-\delta}\right)$ and $B\left(\left.G\right|_{n} ^{n-1}\right) \phi_{P, n-1}=0$, we obtain that

$$
\frac{1}{H_{P, n-1}^{W+}-w_{n+1}}\left(\Delta\left(\left.H_{I}^{W}\right|_{n} ^{n-1}\right)_{\mathrm{mix}}+\left(\left.H_{I}^{W}\right|_{n} ^{n-1}\right)_{\mathrm{quad}}\right) B^{*}\left(\left.G\right|_{n} ^{n-1}\right) \phi_{P, n-1}=O\left(|\lambda|^{3 / 2} \sigma_{n-1}^{2-\delta}\right) .
$$

Next, making use of $\left.|\mathcal{I}|_{n}^{n-1}\left|\leq c_{I}\right| \lambda\right|^{2} \sigma_{n-1}$, (3.72) in Lemma 3.14 combined with an identity analogous to (4.101), Theorem 4.1, (3.70) in Lemma 3.14 (for the $k_{1}+k_{2}$ term below), and the bound in (4.203), we get

$$
\begin{aligned}
& \frac{1}{H_{P, n-1}^{W+}-w_{n+1}}\left(\left.\mathcal{I}\right|_{n} ^{n-1} \cdot \Gamma_{P, n-1}\right) B^{*}\left(\left.G\right|_{n} ^{n-1}\right) \phi_{P, n-1} \\
& \left.\simeq\left(\left.\mathcal{I}\right|_{n} ^{n-1}\right)_{j} \int_{\left(\left.\mathcal{A}\right|_{n} ^{n-1}\right)^{\times 2}}^{\oplus} d^{3} k_{1} d^{3} k_{2} G^{\prime}\left(k_{1}, k_{2}\right)\right|_{n} ^{n-1} \frac{1}{\left[H_{P, n-1}^{W+}\right]_{k_{1}, k_{2}}-w_{n+1}}\left(\Gamma_{P, n-1}+k_{1}+k_{2}\right)_{j} \phi_{P, n-1} \\
& =O\left(|\lambda|^{3} \sigma_{n-1}^{2-2 \delta}\right),
\end{aligned}
$$

where we defined $\left.G^{\prime}\right|_{n} ^{n-1}$ by $\left.G\left(k_{1}, k_{2}\right)\right|_{n} ^{n-1}=:\left.\chi_{\kappa}\left(k_{1}\right) \chi_{\kappa}\left(k_{2}\right)\left|k_{1}\right|^{\bar{\alpha}}\left|k_{2}\right|^{\bar{\alpha}} G^{\prime}\left(k_{1}, k_{2}\right)\right|_{n} ^{n-1}$ and accounted for the factor $\chi_{\kappa}\left(k_{1}\right) \chi_{\kappa}\left(k_{2}\right)\left|k_{1}\right|^{\bar{\alpha}}\left|k_{2}\right|^{\bar{\alpha}}$ by the 'hat' over the integral. Now we look at

$$
\begin{aligned}
& \frac{1}{H_{P, n-1}^{W+}-w_{n+1}}\left(\left.\mathcal{L}\right|_{n} ^{n-1} \cdot \Gamma_{P, n-1}\right) B^{*}\left(\left.G\right|_{n} ^{n-1}\right) \phi_{P, n-1} \\
&=\frac{1}{H_{P, n-1}^{W+}-w_{n+1}}\left(\left(\left.\mathcal{L}\right|_{n} ^{n-1}\right)^{(+)} \cdot \Gamma_{P, n-1}\right) B^{*}\left(\left.G\right|_{n} ^{n-1}\right) \phi_{P, n-1} \\
&+\frac{1}{H_{P, n-1}^{W+}-w_{n+1}}\left(\left(\left.\mathcal{L}\right|_{n} ^{n-1}\right)^{(-)} \cdot \Gamma_{P, n-1}\right) B^{*}\left(\left.G\right|_{n} ^{n-1}\right) \phi_{P, n-1}
\end{aligned}
$$


The direct integral representation, (3.52), (3.72) in Lemma 3.14 combined with an identity analogous to (4.101), Theorem 4.1, (3.70) in Lemma 3.14 (for the $k_{2}+k_{3}$ term below) and the bound in (4.203), give

$$
\begin{aligned}
&(4.266) \simeq-\frac{\lambda}{\sqrt{3 !}} \int_{\left(\left.\mathcal{H}\right|_{n} ^{n-1}\right)^{\times 3}}^{\oplus} d^{3} k_{1} d^{3} k_{2} d^{3} k_{3}\left(\left.\frac{1}{\sqrt{2}\left|k_{1}\right|^{3 / 2}} \frac{k_{1, j}}{\alpha_{P, n-1}\left(\hat{k}_{1}\right)} G^{\prime}\left(k_{2}, k_{3}\right)\right|_{n} ^{n-1} \times\right. \\
&\left.\times \frac{1}{\left[H_{P, n-1}^{W+}\right]_{k_{1}, k_{2}, k_{3}}-w_{n+1}}\left(\Gamma_{P, n-1}+k_{2}+k_{3}\right)_{j} \phi_{P, n-1}+\{\text { perm }\}\right) \\
&=O\left(|\lambda|^{3}|\ln \epsilon|^{3 / 2} \sigma_{n-1}^{2-2 \delta}\right)=O\left(|\lambda|^{2} \sigma_{n-1}^{2-2 \delta}\right),
\end{aligned}
$$

where summation over $j$ is understood. As for (4.267), we have by (3.70) in Lemma 3.14 (used only for the term proportional to $k+k^{\prime}$ below), (3.72) in Lemma 3.14 combined with the identity in (4.101), Theorem 4.1, the bound in (4.203), and the constraint $\sigma_{n} \leq|k|,\left|k^{\prime}\right| \leq \sigma_{n-1}$

$$
\begin{aligned}
(4.267) \simeq & -\left.2 \lambda \int_{\left.\mathcal{A}\right|_{n} ^{n-1}}^{\oplus} d^{3} k^{\prime} \int_{\left.\mathcal{A}\right|_{n} ^{n-1}} d^{3} k \frac{1}{\sqrt{2}|k|^{3 / 2}} \frac{k_{j}}{\alpha_{P, n-1}(\hat{k})} G^{\prime}\left(k, k^{\prime}\right)\right|_{n} ^{n-1} \times \\
& \times \frac{1}{\left[H_{P, n-1}^{W+}\right]_{k^{\prime}}-w_{n+1}}\left(\Gamma_{P, n-1}+k+k^{\prime}\right)_{j} \phi_{P, n-1} \\
= & O\left(|\lambda|^{3} \sigma_{n-1}^{2-\delta}|\ln \epsilon|^{3 / 2}\left(\sigma_{n-1}^{-\delta}+1+\epsilon^{-1}\right)\right)=O\left(\lambda^{2} \sigma_{n-1}^{2-2 \delta}\right),
\end{aligned}
$$

which concludes the proof.

Lemma 4.27. (Control of (4.186) associated with (4.180)) Under the assumptions of Theorem 4.3

$$
R^{\prime}\left(\left.\Delta \Gamma_{P}\right|_{n} ^{n-1}\right)_{i^{\prime}} B^{*}\left(\left.G\right|_{n} ^{n-1}\right) \phi_{P, n-1}:=\frac{1}{H_{P, n-1}^{W+}-z_{n+1}^{\prime}}\left(\left.\Delta \Gamma_{P}\right|_{n} ^{n-1}\right)_{i^{\prime}} B^{*}\left(\left.G\right|_{n} ^{n-1}\right) \phi_{P, n-1}=O\left(|\lambda| \sigma_{n-1}^{1-2 \delta}\right),
$$

where $B^{*}\left(\left.G\right|_{n} ^{n-1}\right)$ appeared in Lemma 4.25 .

Proof. We recall that $\left.\Delta \Gamma_{P}\right|_{n} ^{n-1}=\left.X\right|_{n} ^{n-1}+\left.\mathcal{L}\right|_{n} ^{n-1}$, where $\left.X\right|_{n} ^{n-1}:=-\nabla E_{P, n-1}+\nabla E_{P, n}+\left.\mathcal{I}\right|_{n} ^{n-1}$ is a vector in $\mathbb{R}^{3}$ satisfying

$$
\left.|X|_{n}^{n-1}|\leq c| \lambda\right|^{1 / 4} \sigma_{n-1}^{1-\delta}
$$

Since $\left\|\left(H_{P, n-1}^{W+}-z_{n+1}\right)^{-1}\right\|_{\mathcal{F}_{n}}=O\left(\sigma_{n+1}^{-1}\right)$ and $\left\|\left.G\right|_{n} ^{n-1}\right\|_{2}=O\left(|\lambda|^{2}|\ln \epsilon| \sigma_{n-1}^{1-\delta}\right)=O\left(|\lambda| \sigma_{n-1}^{1-\delta}\right)$ (see (4.220)), we have

$$
\frac{1}{H_{P, n-1}^{W+}-z_{n+1}^{\prime}}\left(\left.X\right|_{n} ^{n-1}\right)_{i} B^{*}\left(\left.G\right|_{n} ^{n-1}\right) \phi_{P, n-1}=O\left(|\lambda|^{5 / 4} \epsilon^{-2} \sigma_{n-1}^{1-2 \delta}\right)=O\left(|\lambda| \sigma_{n-1}^{1-2 \delta}\right) .
$$

Next, we consider the contribution with $\left.\mathcal{L}\right|_{n} ^{n-1}$. We have

$$
\begin{aligned}
\frac{1}{H_{P, n-1}^{W+}-z_{n+1}^{\prime}}\left(\left.\mathcal{L}\right|_{n} ^{n-1}\right)_{i^{\prime}} B^{*}\left(\left.G\right|_{n} ^{n-1}\right) \phi_{P, n-1}= & \frac{1}{H_{P, n-1}^{W+}-z_{n+1}^{\prime}}\left(\left.\mathcal{L}\right|_{n} ^{n-1}\right)_{i^{\prime}}^{(+)} B^{*}\left(\left.G\right|_{n} ^{n-1}\right) \phi_{P, n-1} \\
& +\frac{1}{H_{P, n-1}^{W+}-z_{n+1}^{\prime}}\left(\left.\mathcal{L}\right|_{n} ^{n-1}\right)_{i^{\prime}}^{(-)} B^{*}\left(\left.G\right|_{n} ^{n-1}\right) \phi_{P, n-1} .
\end{aligned}
$$

Making use of (3.70) in Lemma 3.14 and of (4.203), similarly as in (4.253), we have

$$
\begin{aligned}
(4.274) \simeq & \frac{(-\lambda)}{\sqrt{3 !}} \int_{\left(\left.\mathcal{A}\right|_{n} ^{n-1}\right)^{\times 3}}^{\oplus} d^{3} k_{1} d^{3} k_{2} d^{3} k_{3} \\
& \left\{\left.\frac{1}{\sqrt{2}\left|k_{1}\right|^{3 / 2}} \frac{k_{1, i^{\prime}}}{\alpha_{P, n-1}\left(\hat{k}_{1}\right)} G^{\prime}\left(k_{2}, k_{3}\right)\right|_{n} ^{n-1} \frac{1}{\left[H_{P, n-1}^{W+}\right]_{k_{1}, k_{2}, k_{3}}-z_{n+1}^{\prime}} \phi_{P, n-1}+\{\text { perm }\}\right\}, \\
= & O\left(|\lambda|^{3}|\ln \epsilon|^{3 / 2} \sigma_{n-1}^{1-\delta}\right)=O\left(|\lambda|^{2} \sigma_{n-1}^{1-\delta}\right),
\end{aligned}
$$


where we recall that $\left.G\left(k_{1}, k_{2}\right)\right|_{n} ^{n-1}=:\left.\chi_{\kappa}\left(k_{1}\right) \chi_{\kappa}\left(k_{2}\right)\left|k_{1}\right|^{\bar{\alpha}}\left|k_{2}\right|^{\bar{\alpha}} G^{\prime}\left(k_{1}, k_{2}\right)\right|_{n} ^{n-1}$. Next we write, similarly as in (4.256), applying Lemma 3.14, (4.203) and the constraint $\sigma_{n} \leq|k|,\left|k^{\prime}\right| \leq \sigma_{n-1}$

$$
\begin{aligned}
(4.275) & \left.\simeq 2(-\lambda) \int_{\left.\mathcal{H}\right|_{n} ^{n-1}}^{\oplus} d^{3} k^{\prime} \int_{\mathcal{A} n_{n}^{n-1}} d^{3} k \frac{1}{\sqrt{2}|k|^{3 / 2}} \frac{k_{i^{\prime}}}{\alpha_{P, n-1}(\hat{k})} G^{\prime}\left(k, k^{\prime}\right)\right|_{n} ^{n-1} \frac{1}{\left[H_{P, n-1}^{W+}\right]_{k^{\prime}}-z_{n+1}^{\prime}} \phi_{P, n-1} \\
& =O\left(|\lambda|^{3}|\ln \epsilon|^{3 / 2} \epsilon^{-1} \sigma_{n-1}^{1-\delta}\right)=O\left(|\lambda|^{2} \sigma_{n-1}^{1-\delta}\right) .
\end{aligned}
$$

This concludes the proof.

Lemma 4.28. (Control of (4.186) associated with (4.180)) Under the assumptions of Theorem 4.3

$$
R^{\prime}\left(\Gamma_{P, n-1}\right)_{i^{\prime}} B^{*}\left(\left.G\right|_{n} ^{n-1}\right) \phi_{P, n-1}:=\frac{1}{H_{P, n-1}^{W+}-z_{n+1}^{\prime}}\left(\Gamma_{P, n-1}\right)_{i^{\prime}} B^{*}\left(\left.G\right|_{n} ^{n-1}\right) \phi_{P, n-1}=O\left(|\lambda| \sigma_{n-1}^{1-2 \delta}\right),
$$

where $B^{*}\left(\left.G\right|_{n} ^{n-1}\right)$ appeared in Lemma 4.25 .

Proof. Making use of the direct integral representation, of (3.72) in Lemma 3.14 combined with an identity analogous to (4.101) and of (3.70) in Lemma 3.14 (for the $k_{1}+k_{2}$ term below), we get

$$
\begin{aligned}
& \frac{1}{H_{P, n-1}^{W+}-z_{n+1}^{\prime}}\left(\Gamma_{P, n-1}\right)_{i^{\prime}} B^{*}\left(\left.G\right|_{n} ^{n-1}\right) \phi_{P, n-1} \\
& \left.\simeq \sqrt{2} \int_{\left(\left.\mathcal{A}\right|_{n} ^{n-1}\right)^{\times 2}}^{\oplus} d^{3} k_{1} d^{3} k_{2} G^{\prime}\left(k_{1}, k_{2}\right)\right|_{n} ^{n-1} \frac{1}{\left[H_{P, n-1}^{W+}\right]_{k_{1}, k_{2}}-z_{n+1}^{\prime}}\left(\Gamma_{P, n-1}+k_{1}+k_{2}\right)_{i^{\prime}} \phi_{P, n-1} \\
& =O\left(|\lambda|^{2}|\ln \epsilon| \sigma_{n-1}^{1-2 \delta}\right)=O\left(|\lambda| \sigma_{n-1}^{1-2 \delta}\right)
\end{aligned}
$$

where we exploited (4.203) and $G^{\prime}$ is defined below (4.276). This concludes the proof.

Lemma 4.29. Under the assumptions of Theorem 4.3

$$
\frac{1}{\left[H_{P, n-1}^{W+}\right]_{k_{1}, k_{2}}-z_{n+1}} \phi_{P, n-1}=G_{0}\left(k_{1}, k_{2}\right) \phi_{P, n-1}+O\left(\sigma_{n-1}^{-\delta}\right)
$$

where $\sigma_{n} \leq\left|k_{1}\right|,\left|k_{2}\right| \leq \sigma_{n-1}, z_{n+1} \in \gamma_{P, n+1} \cup \tilde{\gamma}_{P, n+1}$ and $G_{0}\left(k_{1}, k_{2}\right)=O\left(\left(\left|k_{1}\right|+\left|k_{2}\right|\right)^{-1}\right)$ is a symmetric numerical function with support in $\left.\overline{\mathcal{A}}\right|_{n} ^{n-1} \times\left.\overline{\mathcal{A}}\right|_{n} ^{n-1}$.

Proof. We recall that

$$
\left[H_{P, n-1}^{W+}\right]_{k_{1}, k_{2}}:=H_{P, n-1}^{W+}+\left(k_{1}+k_{2}\right) \cdot \Gamma_{P, n-1}+\frac{\left(k_{1}+k_{2}\right)^{2}}{2}+\alpha_{P, n-1}\left(\hat{k}_{1}\right)\left|k_{1}\right|+\alpha_{P, n-1}\left(\hat{k}_{2}\right)\left|k_{2}\right| .
$$

Set $a_{P, n-1}\left(k_{1}, k_{2}\right):=\alpha_{P, n-1}\left(\hat{k}_{1}\right)\left|k_{1}\right|+\alpha_{P, n-1}\left(\hat{k}_{2}\right)\left|k_{2}\right|$ and write the following resolvent expansion

$$
\begin{aligned}
\frac{1}{\left[H_{P, n-1}^{W+}\right]_{k_{1}, k_{2}}-z_{n+1}}= & \frac{1}{H_{P, n-1}^{W+}+a_{P, n-1}\left(k_{1}, k_{2}\right)-z_{n+1}} \\
+\frac{1}{\left[H_{P, n-1}^{W+}\right]_{k_{1}, k_{2}}-z_{n+1}}[ & \left.-\left(k_{1}+k_{2}\right) \cdot \Gamma_{P, n-1}-\frac{\left(k_{1}+k_{2}\right)^{2}}{2}\right] \times \\
& \times \frac{1}{H_{P, n-1}^{W+}+a_{P, n-1}\left(k_{1}, k_{2}\right)-z_{n+1}} .
\end{aligned}
$$


The term $G_{0}\left(k_{1}, k_{2}\right) \phi_{P, n-1}$ in (4.280) originates from the first term on the r.h.s. of (4.282), i.e.

$$
G_{0}\left(k_{1}, k_{2}\right):=\left(E_{P, n-1}+\left.\Delta c_{P}\right|_{n} ^{n-1}+a_{P, n-1}\left(k_{1}, k_{2}\right)-z_{n+1}\right)^{-1} .
$$

We have

$$
\begin{aligned}
\left|E_{P, n-1}+\Delta c_{P}\right|_{n}^{n-1}+a_{P, n-1}\left(k_{1}, k_{2}\right)-\operatorname{Re} z_{n+1} \mid \\
\quad \geq a_{P, n-1}\left(k_{1}, k_{2}\right)-\left|E_{P, n-1}-E_{P, n}\right|-\left|\Delta c_{P}\right|_{n}^{n-1} \mid-\frac{\sigma_{n+1}}{3} \\
\quad \geq \frac{1}{3}\left(\left|k_{1}\right|+\left|k_{2}\right|\right)-(1 / 6+1 / 20)\left(\left|k_{1}\right|+\left|k_{2}\right|\right) \geq c^{\prime}\left(\left|k_{1}\right|+\left|k_{2}\right|\right),
\end{aligned}
$$

where $c^{\prime}>0$. Here we made use of the definition $\alpha_{P, n-1}(\hat{k}):=\left(1-\nabla E_{P, n-1} \cdot \hat{k}\right)$, the bounds $\left|\Delta c_{P}\right|_{n}^{n-1}|,| E_{P, n-1}-$ $E_{P, n}\left|\leq(1 / 20) \sigma_{n+1} \leq(1 / 40)\left(\left|k_{1}\right|+\left|k_{2}\right|\right), \nabla E_{P, n} \leq(1 / 3)+c\right| \lambda \mid$ and $\left|\operatorname{Re} z_{n+1}-E_{P, n}\right| \leq \sigma_{n+1} / 3 \leq(1 / 6)\left(\left|k_{1}\right|+\left|k_{2}\right|\right)$. This proves that $G_{0}\left(k_{1}, k_{2}\right)=O\left(\left(\left|k_{1}\right|+\left|k_{2}\right|\right)^{-1}\right)$.

Let us now explain the error term in (4.280). We get from (4.283)-(4.284), (3.72) in Lemma 3.14 combined with an identity analogous to (4.101), Theorem 4.1 and (4.286) that the part of the error term proportional to $\left(k_{1}+k_{2}\right) \cdot \Gamma_{P, n-1}$ is $O\left(\sigma_{n-1}^{-\delta}\right)$. The part proportional to $\left(k_{1}+k_{2}\right)^{2}$ is $O(1)$ by (3.70) in Lemma 3.14.

Lemma 4.30. Under the assumptions of Theorem 4.3 and the inductive hypothesis

$$
\left\|Q_{P, n-1}^{\perp} \frac{1}{H_{P, n-1}^{W+}-w_{n+1}}\left(\Gamma_{P, n-1}\right)_{j} \frac{1}{\left[H_{P, n-1}^{W+}\right]_{k}-w_{n+1}}\left(\Gamma_{P, n-1}\right)_{j^{\prime}} \phi_{P, n-1}\right\|=O\left(\epsilon^{-4} \sigma_{n-1}^{-2 \delta}\right)
$$

for $\sigma_{n} \leq|k| \leq \sigma_{n-1}$.

Proof. To be able to use the induction hypothesis we expand the resolvent of $\left[H_{P, n-1}^{W+}\right]_{k}$ in (4.287) to the second order:

$$
\begin{aligned}
& \frac{1}{\left[H_{P, n-1}^{W+}\right]_{k}-w_{n+1}}:=\frac{1}{H_{P, n-1}^{W+}+k \cdot \Gamma_{P, n-1}+\frac{|k|^{2}}{2}+\alpha_{P, n-1}(\hat{k})|k|-w_{n+1}} \\
= & \frac{1}{H_{P, n-1}^{W+}+\alpha_{P, n-1}(\hat{k})|k|-w_{n+1}} \\
& +\frac{1}{H_{P, n-1}^{W+}+\alpha_{P, n-1}(\hat{k})|k|-w_{n+1}}\left[-k \cdot \Gamma_{P, n-1}-\frac{|k|^{2}}{2}\right] \frac{1}{H_{P, n-1}^{W+}+\alpha_{P, n-1}(\hat{k})|k|-w_{n+1}} \\
& +\frac{1}{\left[H_{P, n-1}^{W+}\right]_{k}-w_{n+1}}\left\{\left[-k \cdot \Gamma_{P, n-1}-\frac{|k|^{2}}{2}\right] \frac{1}{H_{P, n-1}^{W+}+\alpha_{P, n-1}(\hat{k})|k|-w_{n+1}}\right\}^{2} .
\end{aligned}
$$

We set $\tilde{w}_{n+1}:=w_{n+1}-\alpha_{P, n-1}(\hat{k})|k|$ and first consider the contribution of (4.288) to (4.287). We have

$$
\begin{aligned}
& \left\|Q_{P, n-1}^{\perp} \frac{1}{H_{P, n-1}^{W+}-w_{n+1}}\left(\Gamma_{P, n-1}\right)_{j} \frac{1}{H_{P, n-1}^{W+}-\tilde{w}_{n+1}}\left(\Gamma_{P, n-1}\right)_{j^{\prime}} \phi_{P, n-1}\right\| \\
& \leq \sup _{z_{n+1}^{\prime} \in \tilde{\gamma}_{P, n+1}}\left\|Q_{P, n-1}^{\perp} \frac{1}{H_{P, n-1}^{W+}-w_{n+1}}\left(\Gamma_{P, n-1}\right)_{j} \frac{1}{H_{P, n-1}^{W+}-z_{n+1}^{\prime}}\left(\Gamma_{P, n-1}\right)_{j^{\prime}} \phi_{P, n-1}\right\| \leq \sigma_{n-1}^{-2 \delta},
\end{aligned}
$$

where we used that $\alpha_{P, n-1}(\hat{k})|k| \geq 0$, applied the maximal modulus principle and the inductive hypothesis via relation (4.7). 
Now we consider the contribution of the term involving $\Gamma_{P, n-1}$ in (4.289) to (4.287):

$$
\begin{aligned}
& \left\|Q_{P, n-1}^{\perp} \frac{1}{H_{P, n-1}^{W+}-w_{n+1}}\left(\Gamma_{P, n-1}\right)_{j} \frac{1}{H_{P, n-1}^{W+}-\tilde{w}_{n+1}}\left(k \cdot \Gamma_{P, n-1}\right) \frac{1}{H_{P, n-1}^{W+}-\tilde{w}_{n+1}}\left(\Gamma_{P, n-1}\right)_{j^{\prime}} \phi_{P, n-1}\right\| \\
& =\left\|Q_{P, n-1}^{\perp} \frac{1}{H_{P, n-1}^{W+}-w_{n+1}}\left(\Gamma_{P, n-1}\right)_{j} Q_{P, n-1}^{\perp} \frac{1}{H_{P, n-1}^{W+}-\tilde{w}_{n+1}}\left(k \cdot \Gamma_{P, n-1}\right) \frac{1}{H_{P, n-1}^{W+}-\tilde{w}_{n+1}}\left(\Gamma_{P, n-1}\right)_{j^{\prime}} \phi_{P, n-1}\right\| \\
& \quad+\left\|Q_{P, n-1}^{\perp} \frac{1}{H_{P, n-1}^{W+}-w_{n+1}}\left(\Gamma_{P, n-1}\right)_{j} Q_{P, n-1} \frac{1}{H_{P, n-1}^{W+}-\tilde{w}_{n+1}}\left(k \cdot \Gamma_{P, n-1}\right) \frac{1}{H_{P, n-1}^{W+}-\tilde{w}_{n+1}}\left(\Gamma_{P, n-1}\right)_{j^{\prime}} \phi_{P, n-1}\right\| .
\end{aligned}
$$

With the help of (3.48) in Lemma 3.12, the constraint $|k| \leq \sigma_{n-1}$, the maximal modulus principle, and the inductive hypothesis, we have

$$
\text { (4.292) } \leq c \epsilon^{-2} \sigma_{n-1}^{-2 \delta}
$$

Next, applying Theorem 4.1 (twice) in combination with the maximal modulus principle, Lemma 4.31 and $|k| \leq \sigma_{n-1}$ we obtain

$$
(4.293) \leq c \epsilon^{-2} \sigma_{n-1}^{-2 \delta}
$$

Next, we consider the contribution of the term involving $|k|^{2}$ in (4.289) to (4.287). By Theorem 4.1 combined with the maximal modulus principle, Lemma 4.31 and (3.47) in Lemma 3.12, we obtain

$$
\left\|Q_{P, n-1}^{\perp} \frac{1}{H_{P, n-1}^{W}-w_{n+1}}\left(\Gamma_{P, n-1}\right)_{j} \frac{1}{H_{P, n-1}^{W+}-\tilde{w}_{n+1}} \frac{|k|^{2}}{2} \frac{1}{H_{P, n-1}^{W+}-\tilde{w}_{n+1}}\left(\Gamma_{P, n-1}\right)_{j^{\prime}} \phi_{P, n-1}\right\|=O\left(\epsilon^{-4} \sigma_{n-1}^{-\delta}\right) .
$$

Finally, we study the contribution of (4.290) to (4.287). Expression (4.290) consist of four terms which we denote $(k \Gamma, k \Gamma),\left(k \Gamma, k^{2}\right),\left(k^{2}, k \Gamma\right),\left(k^{2}, k^{2}\right)$ in the obvious order. The contribution of $(k \Gamma, k \Gamma)$ to (4.287) can be bounded by

$$
\begin{aligned}
& O\left(\sigma_{n+1}^{-1}\right) \| \frac{1}{\left[H_{P, n-1}^{W+}\right]_{k}-w_{n+1}}\left(k \cdot \Gamma_{P, n-1}\right) \times \\
& \times \frac{1}{H_{P, n-1}^{W+}-\tilde{w}_{n+1}}\left(k \cdot \Gamma_{P, n-1}\right) \frac{1}{H_{P, n-1}^{W+}-\tilde{w}_{n+1}}\left(\Gamma_{P, n-1}\right)_{j^{\prime}} \phi_{P, n-1} \| \\
&=O\left(\sigma_{n+1}^{-1}\right) \| \frac{1}{\left[H_{P, n-1}^{W+}\right]_{k}-w_{n+1}}\left(k \cdot \Gamma_{P, n-1}\right) \times \\
& \times Q_{P, n-1}^{\perp} \frac{1}{H_{P, n-1}^{W+}-\tilde{w}_{n+1}}\left(k \cdot \Gamma_{P, n-1}\right) \frac{1}{H_{P, n-1}^{W+}-\tilde{w}_{n+1}}\left(\Gamma_{P, n-1}\right)_{j^{\prime}} \phi_{P, n-1} \| \\
&+O\left(\sigma_{n+1}^{-1}\right) \| \frac{1}{\left[H_{P, n-1}^{W+}\right]_{k}-w_{n+1}}\left(k \cdot \Gamma_{P, n-1}\right) Q_{P, n-1} \times \\
& \quad \times Q_{P, n-1} \frac{1}{H_{P, n-1}^{W+}-\tilde{w}_{n+1}}\left(k \cdot \Gamma_{P, n-1}\right) \frac{1}{H_{P, n-1}^{W+}-\tilde{w}_{n+1}}\left(\Gamma_{P, n-1}\right)_{j^{\prime}} \phi_{P, n-1} \| .
\end{aligned}
$$

Thus we have by (3.71) in Lemma 3.14, $|k| \leq \sigma_{n-1}$, the maximal modulus principle, and the inductive hypothesis

$$
\text { (4.298) } \leq c \epsilon^{-2} \sigma_{n-1}^{-2 \delta} \text {. }
$$

Now by Lemma 3.14, Theorem 4.1 combined with the maximal modulus principle, $|k| \leq \sigma_{n-1}$ and Lemma 4.31,

$$
\text { (4.299) } \leq c \epsilon^{-4} \sigma_{n-1}^{-2 \delta} \text {. }
$$


Thus altogether

$$
\text { (4.297) } \leq c \epsilon^{-4} \sigma_{n-1}^{-2 \delta}
$$

Now the contribution of $\left(k \Gamma, k^{2}\right)$ to $(4.287)$ has the form

$$
\begin{aligned}
O\left(\sigma_{n+1}^{-1}\right) & \| \frac{1}{\left[H_{P, n-1}^{W+}\right]_{k}-w_{n+1}}\left(k \cdot \Gamma_{P, n-1}\right) \times \\
& \times \frac{1}{H_{P, n-1}^{W+}-\tilde{w}_{n+1}} \frac{|k|^{2}}{2} \frac{1}{H_{P, n-1}^{W+}-\tilde{w}_{n+1}}\left(\Gamma_{P, n-1}\right)_{j^{\prime}} \phi_{P, n-1} \| \leq c \epsilon^{-4} \sigma_{n-1}^{-\delta},
\end{aligned}
$$

where we made use of $\tilde{w}_{n+1}=w_{n+1}-\alpha_{P, n-1}(\hat{k})|k|$, Theorem 4.1 in combination with the maximal modulus principle, (3.71) in Lemma 3.14, Lemma 4.31 and $|k| \leq \sigma_{n-1}$.

Next, the contribution of $\left(k^{2}, k \Gamma\right)$ to $(4.287)$ has the form

$$
\begin{aligned}
& O\left(\sigma_{n+1}^{-1}\right) \| \frac{1}{\left[H_{P, n-1}^{W+}\right]_{k}-w_{n+1}} \frac{|k|^{2}}{2} \times \\
& \quad \times \frac{1}{H_{P, n-1}^{W+}-\tilde{w}_{n+1}}\left(k \cdot \Gamma_{P, n-1}\right) \frac{1}{H_{P, n-1}^{W+}-\tilde{w}_{n+1}}\left(\Gamma_{P, n-1}\right)_{j^{\prime}} \phi_{P, n-1} \| \leq c \epsilon^{-4} \sigma_{n-1}^{-\delta},
\end{aligned}
$$

where we made use of Theorem 4.1 in combination with the maximal modulus principle, (3.70) in Lemma 3.14, $|k| \leq \sigma_{n-1}$ and Lemma 4.31 .

Finally, the contribution of $\left(k^{2}, k^{2}\right)$ to (4.287) has the form

$$
\begin{aligned}
O\left(\sigma_{n+1}^{-1}\right) & \| \frac{1}{\left[H_{P, n-1}^{W+}\right]_{k}-w_{n+1}} \frac{|k|^{2}}{2} \times \\
& \times \frac{1}{H_{P, n-1}^{W+}-\tilde{w}_{n+1}} \frac{|k|^{2}}{2} \frac{1}{H_{P, n-1}^{W+}-\tilde{w}_{n+1}}\left(\Gamma_{P, n-1}\right)_{j^{\prime}} \phi_{P, n-1} \| \leq c \epsilon^{-4} \sigma_{n-1}^{1-\delta},
\end{aligned}
$$

where we made use of the same ingredients as in (4.304). Thus the proof is complete.

Lemma 4.31. Under the assumptions of Theorem 4.3

$$
\begin{aligned}
& \left\|\frac{1}{H_{P, n-1}^{W+}-\tilde{w}_{n+1}}\right\|_{\mathcal{F}_{n}} \leq \frac{c}{\sigma_{n+1}}, \\
& \left\|\frac{1}{H_{P, n-1}^{W+}-\tilde{w}_{n+1}}\left(\Gamma_{P, n-1}\right)_{i}\right\|_{\mathcal{F}_{n}} \leq \frac{c}{\sigma_{n+1}},
\end{aligned}
$$

where $\tilde{w}_{n+1}=w_{n+1}-\alpha_{P, n-1}(\hat{k})|k|, \sigma_{n} \leq|k| \leq \sigma_{n-1}, w_{n+1} \in \gamma_{P, n+1}$.

Proof. We show only (4.307) as the proof of (4.306) is analogous and simpler. We write

$$
\frac{1}{H_{P, n-1}^{W+}-\tilde{w}_{n+1}}\left(\Gamma_{P, n-1}\right)_{i}=\frac{1}{H_{P, n-1}^{W+}-\tilde{w}_{n+1}} Q_{P, n-1}\left(\Gamma_{P, n-1}\right)_{i}+\frac{1}{H_{P, n-1}^{W+}-\tilde{w}_{n+1}} Q_{P, n-1}^{\perp}\left(\Gamma_{P, n-1}\right)_{i} .
$$

The term involving $Q_{P, n-1}$ is estimated by a computation using (4.109), (B.2), (B.12) and $|k|^{-1} \leq \sigma_{n}^{-1} \leq \sigma_{n+1}^{-1}$. As for the term with $Q_{P, n-1}^{\perp}$, making use of the maximal modulus principle and (3.48) in Lemma 3.12, we can write for any $\phi \in \mathcal{F}_{n},\|\phi\| \leq 1$,

$$
\left\|\frac{1}{H_{P, n-1}^{W+}-\tilde{w}_{n+1}} Q_{P, n-1}^{\perp}\left(\Gamma_{P, n-1}\right)_{i} \phi\right\| \leq \sup _{z_{n+1} \in \tilde{\gamma}_{P, n+1}}\left\|\frac{1}{H_{P, n-1}^{W+}-z_{n+1}} Q_{P, n-1}^{\perp}\left(\Gamma_{P, n-1}\right)_{i} \phi\right\| \leq \frac{c}{\sigma_{n+1}},
$$

uniformly in $\phi$. This concludes the proof. 
Lemma 4.32. Under the assumptions of Theorem 4.3

$$
\left.\left.\frac{1}{H_{P, n-1}^{W+}-z_{n+1}^{\prime}}\left(\Gamma_{P, n-1}\right)_{i} \frac{1}{H_{P, n-1}^{W+}-z_{n+1}} \mathcal{L}\right|_{n} ^{n-1} \cdot \mathcal{L}\right|_{n} ^{n-1} \phi_{P, n-1}=O\left(|\lambda| \sigma_{n-1}^{1-\delta}\right)
$$

where $z_{n+1}^{\prime}, z_{n+1} \in \gamma_{P, n+1} \cup \tilde{\gamma}_{P, n+1}$.

Proof. First, we note that

$$
\begin{aligned}
\left(\left.\mathcal{L}\right|_{n} ^{n-1}\right)^{(-)} \cdot\left(\left.\mathcal{L}\right|_{n} ^{n-1}\right)^{(+)} \phi_{P, n-1} & =\lambda^{2} \bar{\int}_{\left.\mathcal{P}\right|_{n} ^{n-1}} d^{3} k \frac{k^{2}}{2|k|^{3} \alpha_{P, n-1}(\hat{k})^{2}} \phi_{P, n-1} \\
& =\phi_{P, n-1} O\left(\lambda^{2}|\ln \epsilon| \sigma_{n-1}^{2}\right) .
\end{aligned}
$$

The first contribution to the expression from the statement of the lemma is therefore

$$
\begin{array}{r}
\frac{1}{H_{P, n-1}^{W+}-z_{n+1}^{\prime}}\left(\Gamma_{P, n-1}\right)_{i} \frac{1}{H_{P, n-1}^{W+}-z_{n+1}}\left(\left(\left.\mathcal{L}\right|_{n} ^{n-1}\right)^{(-)} \cdot\left(\left.\mathcal{L}\right|_{n} ^{n-1}\right)^{(+)}\right) \phi_{P, n-1} \\
=O\left(\lambda^{2}|\ln \epsilon| \epsilon^{-2} \sigma_{n-1}^{1-\delta}\right)=O\left(|\lambda| \sigma_{n-1}^{1-\delta}\right),
\end{array}
$$

where we made use of Theorem 4.1. The remaining contribution is

$$
\begin{aligned}
& \frac{1}{H_{P, n-1}^{W+}-z_{n+1}^{\prime}}\left(\Gamma_{P, n-1}\right)_{i} \frac{1}{H_{P, n-1}^{W+}-z_{n+1}}\left(\left.\mathcal{L}\right|_{n} ^{n-1}\right)^{(+)} \cdot\left(\left.\mathcal{L}\right|_{n} ^{n-1}\right)^{(+)} \phi_{P, n-1} \\
& \simeq \lambda^{2} \sqrt{2} \int_{\left(\left.\mathcal{A}\right|_{n} ^{n-1}\right)^{\times 2}}^{\oplus} d^{3} k_{1} d^{3} k_{2} \frac{1}{\sqrt{2}\left|k_{1}\right|^{3 / 2}} \frac{k_{1, i}}{\alpha_{P, n-1}\left(\hat{k}_{1}\right)} \frac{1}{\sqrt{2}\left|k_{2}\right|^{3 / 2}} \frac{k_{2, i}}{\alpha_{P, n-1}\left(\hat{k}_{2}\right)} \times \\
& \times \frac{1}{\left[H_{P, n-1}^{W+}\right]_{k_{1}, k_{2}}-z_{n+1}^{\prime}}\left(\Gamma_{P, n-1}+k_{1}+k_{2}\right)_{i} \frac{1}{\left[H_{P, n-1}^{W+}\right]_{k_{1}, k_{2}}-z_{n+1}} \phi_{P, n-1} .
\end{aligned}
$$

The part of (4.314) proportional to $k_{1}+k_{2}$ is $O\left(|\lambda|^{2}|\ln \epsilon| \sigma_{n-1}\right)=O\left(|\lambda| \sigma_{n-1}\right)$ by (3.70) in Lemma 3.14. To control the remaining part of (4.314), we write, making use of Lemma 4.29

$$
\begin{aligned}
& \frac{1}{\left[H_{P, n-1}^{W+}\right]_{k_{1}, k_{2}}-z_{n+1}^{\prime}}\left(\Gamma_{P, n-1}\right)_{i} \frac{1}{\left[H_{P, n-1}^{W+}\right]_{k_{1}, k_{2}}-z_{n+1}} \phi_{P, n-1} \\
&=G_{0}\left(k_{1}, k_{2}\right) \frac{1}{\left[H_{P, n-1}^{W+}\right]_{k_{1}, k_{2}}-z_{n+1}^{\prime}}\left(\Gamma_{P, n-1}\right)_{i} \phi_{P, n-1} \\
& \quad+\frac{1}{\left[H_{P, n-1}^{W+}\right]_{k_{1}, k_{2}}-z_{n+1}^{\prime}}\left(\Gamma_{P, n-1}\right)_{i} O\left(\sigma_{n-1}^{-\delta}\right)=O\left(\left(\left|k_{1}\right|+\left|k_{2}\right|\right)^{-1} \sigma_{n-1}^{-\delta}\right),
\end{aligned}
$$

where we applied (3.72) in Lemma 3.14 combined with an identity analogous to (4.101), Theorem 4.1 and the fact that $G_{0}\left(k_{1}, k_{2}\right)=O\left(\left(\left|k_{1}\right|+\left|k_{2}\right|\right)^{-1}\right)$. Substituting (4.315) to (4.314), we obtain altogether

$$
(4.313)=O\left(\lambda^{2}|\ln \epsilon| \sigma_{n-1}^{1-\delta}\right)=O\left(|\lambda| \sigma_{n-1}^{1-\delta}\right),
$$

which completes the proof.

Lemma 4.33. Under the assumptions of Theorem 4.3

$$
\begin{array}{r}
\frac{1}{H_{P, n-1}^{W+}-z_{n+1}^{\prime}}\left(\Gamma_{P, n-1}\right)_{i} \frac{1}{H_{P, n-1}^{W+}-z_{n+1}}\left(\left.\mathcal{L}\right|_{n} ^{n-1} \cdot \Gamma_{P, n-1}\right) \phi_{P, n-1} \\
\quad=b^{*}\left(\left.g_{2, i}\right|_{n} ^{n-1}\right) \phi_{P, n-1}+O\left(|\lambda|^{1 / 4} \sigma_{n-1}^{1-2 \delta}\right),
\end{array}
$$


where $z_{n+1}^{\prime}, z_{n+1} \in \gamma_{P, n+1} \cup \tilde{\gamma}_{P, n+1}$ and $\left.g_{2, i}\right|_{n} ^{n-1}$ is a 3-tuple of functions with support in $\left.\overline{\mathcal{A}}\right|_{n} ^{n-1}$ which satisfy the pointwise bound

$$
\left|g_{2, i}(k)\right|_{n}^{n-1}|\leq| \lambda \mid \frac{c}{|k|^{3 / 2} \sigma_{n-1}^{\delta}}
$$

Proof. We use the direct integral representation

$$
\begin{aligned}
& \left.\frac{1}{H_{P, n-1}^{W+}-z_{n+1}^{\prime}}\left(\Gamma_{P, n-1}\right)_{i} \frac{1}{H_{P, n-1}^{W+}-z_{n+1}} \mathcal{L}\right|_{n} ^{n-1} \cdot \Gamma_{P, n-1} \phi_{P, n-1} \\
\simeq & (-\lambda) \int_{\left.\mathcal{A}\right|_{n} ^{n-1}}^{\oplus} \frac{d^{3} k}{\sqrt{2}|k|^{3 / 2}} \frac{k_{i^{\prime}}}{\alpha_{P, n-1}(\hat{k})} \times \\
& \times \frac{1}{\left[H_{P, n-1}^{W+}\right]_{k}-z_{n+1}^{\prime}}\left(\Gamma_{P, n-1}+k\right)_{i} \frac{1}{\left[H_{P, n-1}^{W+}\right]_{k}-z_{n+1}}\left(\Gamma_{P, n-1}\right)_{i^{\prime}} \phi_{P, n-1} .
\end{aligned}
$$

By (3.72) combined with the identity in (4.101) Lemma 3.14 and Theorem 4.1, the part of (4.319) proportional to $k$ is $O\left(|\lambda||\ln \epsilon|^{1 / 2} \sigma_{n-1}^{1-\delta}\right)=O\left(|\lambda|^{1 / 2} \sigma_{n-1}^{1-\delta}\right)$. We write the remaining part as follows

$$
\begin{aligned}
(-\lambda) & \int_{\left.\mathcal{H}\right|_{n} ^{n-1}}^{\oplus} \frac{d^{3} k}{\sqrt{2}|k|^{3 / 2}} \frac{k_{i^{\prime}}}{\alpha_{P, n-1}(\hat{k})} \times \\
& \times \frac{1}{\left[H_{P, n-1}^{W+}\right]_{k}-z_{n+1}^{\prime}} Q_{P, n-1}^{\perp}\left(\Gamma_{P, n-1}\right)_{i} \frac{1}{\left[H_{P, n-1}^{W+}\right]_{k}-z_{n+1}}\left(\Gamma_{P, n-1}\right)_{i^{\prime}} \phi_{P, n-1} \\
+(-\lambda) & \int_{\left.\mathcal{H}\right|_{n} ^{n-1}}^{\oplus} \frac{d^{3} k}{\sqrt{2}|k|^{3 / 2}} \frac{k_{i^{\prime}}}{\alpha_{P, n-1}(\hat{k})} \times \\
& \times \frac{1}{\left[H_{P, n-1}^{W+}\right]_{k}-z_{n+1}^{\prime}} Q_{P, n-1}\left(\Gamma_{P, n-1}\right)_{i} \frac{1}{\left[H_{P, n-1}^{W+}\right]_{k}-z_{n+1}}\left(\Gamma_{P, n-1}\right)_{i^{\prime}} \phi_{P, n-1} .
\end{aligned}
$$

We have (4.320) $=O\left(|\lambda||\ln \epsilon|^{1 / 2} \epsilon^{-4} \sigma_{n-1}^{1-2 \delta}\right)=O\left(|\lambda|^{1 / 4} \sigma_{n-1}^{1-2 \delta}\right.$, where we made use of (3.72) in Lemma 3.14, and of Lemma 4.30. The analysis of (4.321) is very similar to the discussion of (4.130), thus we can write

$$
(4.321)=b^{*}\left(\left.g_{2, i}\right|_{n} ^{n-1}\right) \phi_{P, n-1}+O\left(|\lambda|^{1 / 2} \sigma_{n-1}^{1-2 \delta}\right),
$$

where $\left.g_{2, i}\right|_{n} ^{n-1}$ is a 3-tuple of functions with support in $\left.\overline{\mathcal{A}}\right|_{n} ^{n-1}$ which satisfy the pointwise bound $\left|g_{2, i^{\prime}}(k)\right|_{n}^{n-1} \mid \leq$ $|\lambda| \frac{c}{|k|^{3 / 2} \sigma_{n-1}^{\delta}}$. This completes the proof.

\section{$5 \quad$ Proof of Theorem 2.1}

\subsection{Proof of Theorem $2.1(\mathrm{c})$}

An immediate consequence of Theorem 4.1 (and its proof) is the convergence of the sequence $\left\{\phi_{P, n}\right\}_{n \in \mathbb{N}_{0}}$ to the limiting non-zero vector $\phi_{P}$, which is the ground state of the transformed Hamiltonian with removed infrared cut-off $H_{P, n=\infty}^{W}$. To study the properties of this sequence, we define an antiunitary $J$ on $\mathcal{F}$ as follows: for $\varphi \in \mathcal{F}$ with momentum wave functions $\left\{\varphi^{q}\left(k_{1}, \ldots, k_{q}\right)\right\}_{q \in \mathbb{N}_{0}}$ we set

$$
J\left\{\varphi^{q}\left(k_{1}, \ldots, k_{q}\right)\right\}_{q \in \mathbb{N}_{0}}:=\left\{\overline{\varphi^{q}\left(k_{1}, \ldots, k_{q}\right)}\right\}_{q \in \mathbb{N}_{0}},
$$

where $J \Omega=\Omega$ is understood. Now we prove the following corollary of Theorem 4.1. 
Corollary 5.1. Let $\left\{\phi_{P, n}\right\}_{n \in \mathbb{N}_{0}}$ be the sequence of vectors appearing in Theorem 4.1 and denote $\check{\phi}_{P, n}:=\phi_{P, n} /\left\|\phi_{P, n}\right\|$. Then, under the assumptions of Theorem 4.1:

(a) $\left\|\phi_{P, n+1}-\phi_{P, n}\right\| \leq 2|\lambda|^{1 / 4} \sigma_{n}^{1 / 2}$ and $\left\|\check{\phi}_{P, n+1}-\check{\phi}_{P, n}\right\| \leq 8|\lambda|^{1 / 4} \sigma_{n}^{1 / 2}$. Therefore, the sequence $\left\{\phi_{P, n}\right\}_{n \in \mathbb{N}_{0}}$ (resp. $\left.\left\{\check{\phi}_{P, n}\right\}_{n \in \mathbb{N}_{0}}\right)$ is convergent as $n \rightarrow \infty$ to the non-zero limiting vector $\phi_{P}\left(\right.$ resp. $\left.\check{\phi}_{P}\right)$.

(b) $\left\langle\check{\phi}_{P, n}, \Omega\right\rangle>1 / 2$.

(c) Let $Q_{P, n}$ be the spectral projection onto the ground state energy of $H_{P, n}^{W}$; see (3.38). Then $\breve{\phi}_{P, n}=$ $Q_{P, n} \Omega /\left\|Q_{P, n} \Omega\right\|$.

Proof. The estimates in part (a) follow from Theorem 4.1 ii), iii), and formula (C.43). The convergence of the sequences to non-zero vectors then follows by a telescopic argument and Theorem 4.1 iii).

To prove part (b), we recall that $\phi_{P, 0}:=\Omega$ and note that by part (a)

$$
\left\|\Omega-\check{\phi}_{P, n}\right\| \leq 8|\lambda|^{1 / 4} \sum_{n=0}^{\infty} \sigma_{n}^{1 / 2}<1
$$

for $\left|\lambda_{0}\right|$ sufficiently small, so that we obtain $\operatorname{Re}\left\langle\check{\phi}_{P, n}, \Omega\right\rangle>1 / 2$. Noting that, by definition (3.18), $J \check{\phi}_{P, n}=\check{\phi}_{P, n}$, we deduce that $\left\langle\breve{\phi}_{P, n}, \Omega\right\rangle$ is real and complete the proof of part (b) of the corollary.

To prove part (c), we note that by $\left\langle\check{\phi}_{P, n}, \Omega\right\rangle>1 / 2$, the uniqueness of the ground state of $H_{P, n}^{W}$, and $J Q_{P, n} \Omega=$ $Q_{P, n} \Omega$ we have that $\check{\phi}_{P, n}=s(n) Q_{P, n} \Omega /\left\|Q_{P, n} \Omega\right\|$, where $s(n) \in\{ \pm 1\}$. Clearly, $s(0)=1$. To show that $s(n)=1$ for all $n \geq 0$, suppose the opposite, i.e. that $s\left(n_{*}\right)=-1$ for some $n_{*} \in \mathbb{N}$. Then

$$
1>\left\|\Omega-\check{\phi}_{P, n_{*}}\right\| \geq\left\langle\Omega, \Omega-\check{\phi}_{P, n_{*}}\right\rangle=1-\left\langle\Omega, \breve{\phi}_{P, n_{*}}\right\rangle=1+\left\|Q_{P, n_{*}} \Omega\right\|,
$$

which is a contradiction.

Now we describe the passage from the discrete to the continuous infrared cut-off, following [29]. For any $\delta \in(0,1 / 4)$ we fix a sequence of discrete cut-offs $\rho_{n}:=\left(\epsilon^{*}(\delta)\right)^{n}$ as specified in Theorem 4.3. Now for any given (continuous) infrared cut-off $\sigma \in\left(0, \epsilon^{*}(\delta)\right]$ there is exactly one $n_{\sigma} \in \mathbb{N}$ s.t. $\rho_{n_{\sigma}+1}<\sigma \leq \rho_{n_{\sigma}}$. Defining $\epsilon_{\sigma}:=\sigma^{1 / n_{\sigma}}$, we obtain a new sequence of discrete cut-offs $\sigma_{n}:=\epsilon_{\sigma}^{n}$ with the property $\sigma_{n_{\sigma}}=\sigma$. Clearly, $\epsilon^{*}(\delta)^{1+1 / n_{\sigma}}<\epsilon_{\sigma} \leq \epsilon^{*}(\delta)$, hence $\epsilon_{\sigma} \in\left(\epsilon^{*}(\delta)^{2}, \epsilon^{*}(\delta)\right]$ and $\epsilon_{\sigma} \rightarrow \epsilon^{*}(\delta)$ for $\sigma \rightarrow 0$.

Definition 5.2. For any sequence $\left\{A_{n}^{(\epsilon)}\right\}_{n \in \mathbb{N}}$ of quantities depending on the discrete cut-off (where we write the dependence on $\epsilon$ explicitly), we can set $A_{\sigma}:=A_{n_{\sigma}}^{\left(\epsilon_{\sigma}\right)}$. By applying this rule we recover $H_{P, \sigma}$, introduced in (2.4), and also define $W_{P, \sigma}, H_{P, \sigma}^{W}, E_{P, \sigma}, \Gamma_{P, \sigma}, \phi_{P, \sigma}, \hat{\phi}_{P, \sigma}, Q_{P, \sigma}, \hat{Q}_{P, \sigma}, \bar{Q}_{P, \sigma}$ which will be used below.

We cannot apply this rule to $\gamma_{P, n}$, since it depends both on $n$ and $n-1$. Instead, we declare

$$
\gamma_{P, \sigma}:=\left\{w \in \mathbb{C}|| w-E_{P, \sigma} \mid=\frac{\sigma}{6}\right\} .
$$

As for the ground state vector of $H_{P, \sigma}$, it will be convenient to set its phase as follows:

Definition 5.3. We define $\check{\psi}_{P, \sigma}:=W_{P, \sigma}^{*} \check{\phi}_{P, \sigma}$, where $\check{\phi}_{P, \sigma}:=\phi_{P, \sigma} /\left\|\phi_{P, \sigma}\right\|$, and $\phi_{P, \sigma}:=\phi_{P, n_{\sigma}}^{\left(\epsilon_{\sigma}\right)}$ was constructed in Corollary 3.5. In view of Corollary 5.1 (c), $\check{\phi}_{P, \sigma}$ can equivalently be defined as

$$
\check{\phi}_{P, \sigma}:=\frac{\oint_{\gamma_{P, \sigma}} d w \frac{1}{H_{P, \sigma}^{W}-w} \Omega}{\left\|\oint_{\gamma_{P, \sigma}} d w \frac{1}{H_{P, \sigma}^{W}-w} \Omega\right\|} .
$$


After this preparation, we proceed to the proof of Theorem 2.1 (c). To this end, we establish a counterpart of Corollary 5.1 (a) for the continuous cut-off, that is showing that $\left\|\check{\phi}_{P, \bar{\sigma}}-\breve{\phi}_{P, \sigma}\right\| \leq 8|\lambda|^{1 / 4} \sigma^{1 / 2}$ for $0<\bar{\sigma}<$ $\sigma \leq \epsilon^{*}(\delta)$. We cannot apply the arguments from the proof of the corollary and Theorem 4.1 directly, because $\epsilon_{\sigma} \neq \epsilon_{\bar{\sigma}}$ in general. Instead, we proceed as follows: we find $n \in \mathbb{N}_{0}$ s.t. $\bar{\sigma} \in\left[\sigma_{n+1}, \sigma_{n}\right)$ and we define $\hat{H}_{P, \bar{\sigma}}^{W}$, $\hat{Q}_{P, \bar{\sigma}}$ as indicated in Definition 5.2 using the sequence of cut-offs $\left\{\epsilon_{\bar{\sigma}}^{n}\right\}_{n=0}^{\infty}$. By adapting our considerations from Subsection 3.2, we can write

$$
\hat{H}_{P, \bar{\sigma}}=H_{P, n}^{W}+\left.\Delta c_{P}\right|_{\bar{\sigma}} ^{n}+\left.H_{I}^{W}\right|_{\bar{\sigma}} ^{n}
$$

where

$$
\left.H_{I}^{W}\right|_{\bar{\sigma}} ^{n}:=\left(\left.\mathcal{L}\right|_{\bar{\sigma}} ^{n}+\left.\mathcal{I}\right|_{\bar{\sigma}} ^{n}\right) \cdot \Gamma_{P, n}+\Delta\left(\left.H_{I}^{W}\right|_{\bar{\sigma}} ^{n}\right)_{\mathrm{mix}}+\left(\left.H_{I}^{W}\right|_{\bar{\sigma}} ^{n}\right)_{\mathrm{quad}}
$$

and the quantities $\left.\mathcal{L}\right|_{\bar{\sigma}} ^{n},\left.\mathcal{I}\right|_{\bar{\sigma}} ^{n},\left.\Delta c_{P}\right|_{\bar{\sigma}} ^{n}, \Delta\left(\left.H_{I}^{W}\right|_{\bar{\sigma}} ^{n}\right)_{\text {mix }}, \Delta\left(\left.H_{I}^{W}\right|_{\bar{\sigma}} ^{n}\right)_{\text {mix }},\left(\left.H_{I}^{W}\right|_{\bar{\sigma}} ^{n}\right)_{\text {quad }}$ are obtained by replacing $\sigma_{n+1}=\epsilon_{\sigma}^{n+1}$ with $\bar{\sigma}$ in their counterparts from Section 3. Using that $\bar{\sigma} \geq \sigma_{n+1}$, it is easy to check that these expressions satisfy the estimates from Lemma 3.12 and that considerations about direct integral representations from Subsection 3.4 can be adapted to the present situation. Furthermore, the discussion from Appendix D can be repeated for the modified cut-off as follows. We set

$$
\begin{aligned}
& W_{P, \bar{\sigma}}:=\exp \left(-\lambda \int_{\bar{\sigma}}^{k} \frac{d^{3} k}{\sqrt{2}|k|^{\frac{3}{2}} \alpha_{P, \bar{\sigma}}(\hat{k})}\left\{b(k)-b^{*}(k)\right\}\right), \\
& \widetilde{W}_{P, \bar{\sigma}}^{\prime}:=\exp \left(-\lambda \int_{\bar{\sigma}}^{k} \frac{d^{3} k}{\sqrt{2}|k|^{\frac{3}{2}} \alpha_{P, n}(\hat{k})}\left\{b(k)-b^{*}(k)\right\}\right),
\end{aligned}
$$

where $\alpha_{P, \bar{\sigma}}(\hat{k}):=\left(1-\hat{k} \cdot \nabla E_{P, \bar{\sigma}}\right)$, and define by analogy with (3.18)

$$
\hat{\phi}_{P, \bar{\sigma}}^{\prime}:=\hat{Q}_{P, \bar{\sigma}} \phi_{P, n}, \quad \phi_{P, \bar{\sigma}}^{\prime}:=W_{P, \bar{\sigma}}\left(\widetilde{W}_{P, \bar{\sigma}}^{\prime}\right)^{*} \hat{\phi}_{P, \bar{\sigma}}^{\prime},
$$

where we use the prime to distinguish these vectors from $\hat{\phi}_{P, \bar{\sigma}}$ and $\phi_{P, \bar{\sigma}}$ defined using the rule in Definition 5.2. Now by repeating the arguments from Lemmas D.5, D.6 we obtain:

Lemma 5.4. Let $|P| \leq P_{\max }=1 / 3$ and $|\lambda| \in\left(0, \lambda_{0}\right]$. Then

$$
\begin{gathered}
\left|\nabla E_{P, \bar{\sigma}}-\nabla E_{P, n}\right| \leq c_{1}\left[\lambda^{2} \sigma_{n}+\left\|\frac{\hat{\phi}_{P, \bar{\sigma}}^{\prime}}{\left\|\hat{\phi}_{P, \bar{\sigma}}^{\prime}\right\|}-\frac{\phi_{P, n}}{\left\|\phi_{P, n}\right\|}\right\|\right], \\
\left\|\left(W_{P, \bar{\sigma}}\left(\widetilde{W}_{P, \bar{\sigma}}^{\prime}\right)^{*}-1\right) \hat{\phi}_{P, \bar{\sigma}}^{\prime}\right\| \leq c|\lambda|\left|\nabla E_{P, \bar{\sigma}}-\nabla E_{P, n}\|\ln \bar{\sigma} \mid\| \hat{\phi}_{P, \bar{\sigma}}^{\prime} \|,\right. \\
\left\|\left(W_{P, \bar{\sigma}} W_{P, n}^{*}-1\right) \hat{\phi}_{P, \bar{\sigma}}^{\prime}\right\| \leq c|\lambda|\left|\nabla E_{P, \bar{\sigma}}-\nabla E_{P, n}\|\ln \bar{\sigma} \mid\| \hat{\phi}_{P, \bar{\sigma}}^{\prime} \|\right. \\
+c|\lambda|(\bar{\alpha})^{-1} \sigma_{n}^{\bar{\alpha}}\left\|\hat{\phi}_{P, \bar{\sigma}}^{\prime}\right\|,
\end{gathered}
$$

where (only) for (5.13) we assumed $\bar{\alpha}>0$ and (5.12), (5.13) also hold after replacing $\hat{\phi}_{P, \bar{\sigma}}^{\prime}$ with $\phi_{P, \bar{\sigma}}^{\prime}$.

Now we are ready to prove the following proposition:

Proposition 5.5. Let $|P| \leq P_{\max }=1 / 3$ and $|\lambda| \in\left(0, \lambda_{0}\right]$. Then

(a) For $\bar{\alpha} \geq 0,\left\|\check{\phi}_{P, \bar{\sigma}}-\check{\phi}_{P, n}\right\| \leq c|\lambda|^{1 / 4} \sigma_{n}^{1 / 2}$,

(b) For $\bar{\alpha}>0,\left\|\breve{\psi}_{P, \bar{\sigma}}-\breve{\psi}_{P, n}\right\| \leq c|\lambda|^{1 / 8}(\bar{\alpha})^{-1} \sigma_{n}^{\bar{\alpha}}$,

where $\check{\phi}_{P, \bar{\sigma}}:=Q_{P, \bar{\sigma}} \Omega /\left\|Q_{P, \bar{\sigma}} \Omega\right\|$ and $\check{\psi}_{P, \bar{\sigma}}:=W_{P, \bar{\sigma}}^{*} \check{\phi}_{P, \bar{\sigma}}$ according to Definition 5.3. 
Proof. Using the information in (5.6)-(5.12) and repeating the steps from the proof of Claim ii) and Claim iii) of Theorem 4.1, we obtain for some $0<\delta<1 / 4$

$$
\left\|\hat{\phi}_{P, \bar{\sigma}}^{\prime}-\phi_{P, n}\right\| \leq|\lambda|^{\frac{1}{4}} \sigma_{n}^{1-\delta}, \quad\left\|\phi_{P, \bar{\sigma}}^{\prime}-\hat{\phi}_{P, \bar{\sigma}}^{\prime}\right\| \leq|\lambda|^{1 / 4} \bar{\sigma}^{1 / 2}, \quad\left\|\phi_{P, \bar{\sigma}}^{\prime}\right\| \geq(1 / 2)
$$

(We note that the application of Claim i) of Theorem 4.1 within the above discussion is unproblematic, since it relies on $\bar{\sigma}$-independent ${ }^{1}$ ingredients $H_{P, n}^{W}$ and $\Gamma_{P, n}$ in (5.6)). Now setting $\breve{\phi}_{P, \bar{\sigma}}^{\prime}:=\phi_{P, \bar{\sigma}}^{\prime} /\left\|\phi_{P, \bar{\sigma}}^{\prime}\right\|$, we immediately get

$$
\left\|\check{\phi}_{P, \bar{\sigma}}^{\prime}-\check{\phi}_{P, n}\right\| \leq 8|\lambda|^{1 / 4} \sigma_{n}^{1 / 2} \text {. }
$$

Let us now prove that $\check{\phi}_{P, \bar{\sigma}}^{\prime}=\check{\phi}_{P, \bar{\sigma}}$ : We recall from Corollary 5.1 that $\check{\phi}_{P, n}=Q_{P, n} \Omega /\left\|Q_{P, n} \Omega\right\|$, where $Q_{P, n}$ is the ground state projection of $H_{P, n}^{W}$. Since, by construction, $J \check{\phi}_{P, \bar{\sigma}}^{\prime}=\check{\phi}_{P, \bar{\sigma}}^{\prime}$, we have $\breve{\phi}_{P, \bar{\sigma}}^{\prime}=( \pm) Q_{P, \bar{\sigma}} \Omega /\left\|Q_{P, \bar{\sigma}} \Omega\right\|$. Suppose there is a (-) sign in the last formula. Then, by (5.15) and Corollary 5.1 (b)

$$
8|\lambda|^{1 / 4} \sigma_{n}^{1 / 2} \geq\left\|\frac{Q_{P, \bar{\sigma}} \Omega}{\left\|Q_{P, \bar{\sigma}} \Omega\right\|}+\frac{Q_{P, n} \Omega}{\left\|Q_{P, n} \Omega\right\|}\right\| \geq\left\langle\Omega, \check{\phi}_{P, \bar{\sigma}}\right\rangle+\left\langle\Omega, \check{\phi}_{P, n}\right\rangle>1,
$$

where we have used that both $\left\langle\Omega, \check{\phi}_{P, \bar{\sigma}}\right\rangle$ and $\left\langle\Omega, \check{\phi}_{P, n}\right\rangle$ are larger than $\frac{1}{2}$. As this is a contradiction (possibly after reducing $\left.\left|\lambda_{0}\right|\right)$ we obtain that $\check{\phi}_{P, \bar{\sigma}}^{\prime}=Q_{P, \bar{\sigma}} \Omega /\left\|Q_{P, \bar{\sigma}} \Omega\right\| \equiv \breve{\phi}_{P, \bar{\sigma}}$. Together with (5.15), this concludes the proof of part (a) of the proposition.

Proceeding to part (b), we write, making use of (5.15) and Lemma 5.4,

$$
\begin{aligned}
\left\|\check{\psi}_{P, \bar{\sigma}}-\check{\psi}_{P, n}\right\| & \leq\left\|\left(W_{P, \bar{\sigma}} W_{P, n}^{*}-1\right) \check{\phi}_{P, \bar{\sigma}}\right\|+\left\|\check{\phi}_{P, \bar{\sigma}}-\check{\phi}_{P, n}\right\| \\
& \leq c\left[\lambda^{2} \sigma_{n}+4|\lambda|^{1 / 4} \sigma_{n}^{1-\delta}\right]|\ln \bar{\sigma}|+c|\lambda|(\bar{\alpha})^{-1} \sigma_{n}^{\bar{\alpha}}+8|\lambda|^{1 / 4} \sigma_{n}^{1 / 2} \\
& \leq c|\lambda|^{1 / 8}(\bar{\alpha})^{-1} \sigma_{n}^{\bar{\alpha}},
\end{aligned}
$$

where we used that $0<\bar{\alpha} \leq 1 / 2$. This concludes the proof.

We recall that, according to Definition 5.2, $\breve{\psi}_{P, n_{\sigma}}=\breve{\psi}_{P, n_{\sigma}}^{\left(\epsilon_{\sigma}\right)}=: \breve{\psi}_{P, \sigma}$ and $\sigma_{n_{\sigma}}=\sigma$. Then, by the telescopic argument (to step from $n_{\sigma}$ to $n$ ) Proposition 5.5 yields immediately the following corollary. Its second part gives Theorem 2.1 (c).

Corollary 5.6. For $|\lambda| \in\left(0, \lambda_{0}\right]$ and $0<\bar{\sigma}<\sigma \leq \epsilon^{*}(\delta)$ we have

(a) For $\bar{\alpha} \geq 0,\left\|\check{\phi}_{P, \bar{\sigma}}-\check{\phi}_{P, \sigma}\right\| \leq c|\lambda|^{1 / 4} \sigma^{1 / 2}$,

(b) For $\bar{\alpha}>0$, $\left\|\breve{\psi}_{P, \bar{\sigma}}-\breve{\psi}_{P, \sigma}\right\| \leq c|\lambda|^{1 / 8}(\bar{\alpha})^{-1} \sigma^{\bar{\alpha}}$.

Consequently, the limits $\check{\phi}_{P}:=\lim _{\sigma \rightarrow 0} \check{\phi}_{P, \sigma}$ and $\breve{\psi}_{P}:=\lim _{\sigma \rightarrow 0} \breve{\psi}_{P, \sigma}$ exist. (The latter only for $\bar{\alpha}>0$ ).

\subsection{Proof of Theorem 2.1 (b)}

By a straightforward modification of the discussion from Lemmas A.12-A.14 we obtain $\left|E_{P, \bar{\sigma}}-E_{P, n}\right| \leq c|\lambda|^{2} \sigma_{n}$, which by the telescopic argument gives $\left|E_{P, \bar{\sigma}}-E_{P, \sigma}\right| \leq c|\lambda|^{2} \sigma$ for $0<\bar{\sigma}<\sigma \leq 1$. The statement about convexity of $S \ni P \mapsto E_{P}$ is proven in Appendix E.

\footnotetext{
${ }^{1}$ To be more precise they are not explicitly $\bar{\sigma}$-dependent, indeed the value $n$ depends on $\sigma$ and $\bar{\sigma}$.
} 


\subsection{Proof of Theorem 2.1 (a)}

We start with the following corollary of Theorems 4.1, 4.3.

Corollary 5.7. There is $\lambda_{0}>0$ and $\delta_{\lambda_{0}} \in(0,1 / 4)$ s.t. for all $\lambda \in\left(0, \lambda_{0}\right], \sigma \in\left(0, \epsilon^{*}(\delta)\right]$ and $P \leq P_{\max }=1 / 3$ the following estimates hold true

$$
\begin{aligned}
& \left\|\frac{1}{H_{P, \sigma}^{W}-E_{P, \sigma}}\left(\Gamma_{P, \sigma}\right)_{i} \check{\phi}_{P, \sigma}\right\| \leq \frac{1}{\sigma^{\delta_{\lambda_{0}}}} \\
& \left\|\frac{Q_{P, \sigma}^{\perp}}{H_{P, \sigma}^{W}-E_{P, \sigma}}\left(\Gamma_{P, \sigma}\right)_{i} \frac{1}{H_{P, \sigma}^{W}-E_{P, \sigma}}\left(\Gamma_{P, \sigma}\right)_{i^{\prime}} \check{\phi}_{P, \sigma}\right\| \leq \frac{1}{\sigma^{2 \delta_{\lambda_{0}}}} .
\end{aligned}
$$

Clearly, (5.18), (5.19) remain valid after replacing $\lambda_{0}$ by some $\tilde{\lambda}_{0} \in\left(0, \lambda_{0}\right]$. The resulting function $\tilde{\lambda}_{0} \rightarrow \delta_{\tilde{\lambda}_{0}}$ can be chosen s.t. $\lim _{\tilde{\lambda}_{0} \rightarrow 0} \delta_{\tilde{\lambda}_{0}}=0$.

Proof. We discuss only (5.19), since the treatment of (5.18) is analogous and simpler. Fix $\delta \in(0,1 / 4), \epsilon^{*}(\delta)$ and $\lambda_{0}^{*}\left(\epsilon^{*}(\delta)\right)$ as in Theorem 4.3. Now, as described above Definition 5.2, for any $\sigma \in\left(0, \epsilon^{*}(\delta)\right]$ we obtain $\epsilon_{\sigma} \in\left(\epsilon^{*}(\delta)^{2}, \epsilon^{*}(\delta)\right]$ and $n_{\sigma} \in \mathbb{N}$ s.t. $\sigma=\epsilon_{\sigma}^{n_{\sigma}}$. Then, using also the maximal modulus principle, we obtain from Theorem 4.3

$$
\left\|\frac{Q_{P, \sigma}^{\perp}}{H_{P, \sigma}^{W}-E_{P, \sigma}}\left(\Gamma_{P, \sigma}\right)_{i} \frac{1}{H_{P, \sigma}^{W}-E_{P, \sigma}}\left(\Gamma_{P, \sigma}\right)_{i^{\prime}} \breve{\phi}_{P, \sigma}\right\| \leq \frac{1}{\sigma^{2 \delta}}
$$

for all $|\lambda| \leq \epsilon_{\sigma}^{8}$, hence for all $|\lambda| \leq \epsilon^{*}(\delta)^{16}$. Now we define the function of $0<\tilde{\lambda}_{0} \leq \lambda_{0}^{*}\left(\epsilon^{*}(\delta)\right)$

$$
\delta\left(\tilde{\lambda}_{0}\right):=\inf \left\{\delta \in(0,1 / 4) \mid \tilde{\lambda}_{0} \leq \epsilon^{*}(\delta)^{16}\right\} .
$$

Since for any $\delta \in(0,1 / 4)$ we can find a non-zero $\epsilon^{*}(\delta)$ we have $\lim _{\tilde{\lambda}_{0} \rightarrow 0} \delta\left(\tilde{\lambda}_{0}\right)=0$. Thus setting e.g. $\delta_{\tilde{\lambda}_{0}}:=$ $\delta\left(\tilde{\lambda}_{0}\right)+\tilde{\lambda}_{0}$ (to account for the fact that the infimum may be outside of the set) we conclude the proof.

Now we compute the first and the second derivative with respect to $P$ of the normalized ground state vector $\breve{\psi}_{P, \sigma}$ of the Hamiltonian $H_{P, \sigma}$ introduced in Definition 5.3. Since $\breve{\psi}_{P, \sigma}=\bar{Q}_{P, \sigma} W_{P, \sigma}^{*} \breve{\phi}_{P, \sigma} /\left\|\bar{Q}_{P, \sigma} W_{P, \sigma}^{*} \breve{\phi}_{P, \sigma}\right\|$, the families of operators $\left\{H_{P, \sigma}\right\}_{P \in \mathbb{R}^{3}},\left\{H_{P, \sigma}^{W}\right\}_{P \in \mathbb{R}^{3}}$ are analytic of type $\mathrm{A}$, and the eigenvalue $E_{P, \sigma}$ is isolated on the relevant subspaces (see Proposition 3.2), we conclude that the derivatives of $\breve{\psi}_{P, \sigma}$ w.r.t. $P$ exist. We write

$$
\check{\psi}_{P, \sigma}=\oint_{\gamma_{P, \sigma}} d w \frac{1}{H_{P, \sigma}-w} \check{\psi}_{P, \sigma}
$$

and note that the r.h.s is invariant under small changes of the integration contour. Thus we can write

$$
\partial_{i} \breve{\psi}_{P, \sigma}=\oint_{\gamma_{P, \sigma}} d w \frac{1}{H_{P, \sigma}-w}(-)\left(P-P_{\mathrm{f}}\right)_{i} \frac{1}{H_{P, \sigma}-w} \breve{\psi}_{P, \sigma}+\breve{\psi}_{P, \sigma}\left\langle\check{\psi}_{P, \sigma}, \partial_{i} \check{\psi}_{P, \sigma}\right\rangle,
$$

where we set $\partial_{i}:=\partial_{P_{i}}$. By normalization of $\breve{\psi}_{P, \sigma}$ we have $\partial_{i}\left\|\breve{\psi}_{P, \sigma}\right\|^{2}=0$, i.e., $\operatorname{Re}\left\langle\breve{\psi}_{P, \sigma}, \partial_{i} \breve{\psi}_{P, \sigma}\right\rangle=0$. Moreover, by Definition 5.3

$$
\left\langle\check{\psi}_{P, \sigma}, \partial_{i} \breve{\psi}_{P, \sigma}\right\rangle=\left\langle J \breve{\psi}_{P, \sigma}, J \partial_{i} \check{\psi}_{P, \sigma}\right\rangle=\overline{\left\langle\check{\psi}_{P, \sigma}, \partial_{i} \check{\psi}_{P, \sigma}\right\rangle},
$$

hence also $\operatorname{Im}\left\langle\breve{\psi}_{P, \sigma}, \partial_{i} \breve{\psi}_{P, \sigma}\right\rangle=0$. Thus we obtain

$$
\partial_{i} \check{\psi}_{P, \sigma}=\oint_{\gamma_{P, \sigma}} d w \frac{1}{H_{P, \sigma}-w}(-)\left(P-P_{\mathrm{f}}\right)_{i} \frac{1}{H_{P, \sigma}-w} \breve{\psi}_{P, \sigma} .
$$


Therefore

$$
\begin{aligned}
\partial_{i^{\prime}} \partial_{i} \check{\psi}_{P, \sigma}= & \left(\oint_{\gamma_{P, \sigma}} d w \frac{1}{H_{P, \sigma}-w}(-)\left(P-P_{\mathrm{f}}\right)_{i^{\prime}} \frac{1}{H_{P, \sigma}-w}(-)\left(P-P_{\mathrm{f}}\right)_{i} \frac{1}{H_{P, \sigma}-w} \breve{\psi}_{P, \sigma}+\left\{i \leftrightarrow i^{\prime}\right\}\right) \\
& +\left(\oint_{\gamma_{P, \sigma}} d w \frac{1}{H_{P, \sigma}-w}(-)\left(P-P_{\mathrm{f}}\right)_{i} \frac{1}{H_{P, \sigma}-w}\right)\left(\oint_{\gamma_{P, \sigma}} d \mu \frac{1}{H_{P, \sigma}-\mu}(-)\left(P-P_{\mathrm{f}}\right)_{i^{\prime}} \frac{1}{H_{P, \sigma}-\mu}\right) \check{\psi}_{P, \sigma} \\
= & \left(\oint_{\gamma_{P, \sigma}} d w \frac{1}{H_{P, \sigma}-w}(-)\left(P-P_{\mathrm{f}}\right)_{i^{\prime}} \frac{1}{H_{P, \sigma}-w}(-)\left(P-P_{\mathrm{f}}\right)_{i} \frac{1}{H_{P, \sigma}-w} \check{\psi}_{P, \sigma}+\left\{i \leftrightarrow i^{\prime}\right\}\right) \\
& +\breve{\psi}_{P, \sigma}\left\langle\check{\psi}_{P, \sigma},(-)\left(P-P_{\mathrm{f}}\right)_{i} \frac{\bar{Q}_{P, \sigma}^{\perp}}{\left(H_{P, \sigma}-E_{P, \sigma}\right)^{2}}(-)\left(P-P_{\mathrm{f}}\right)_{i^{\prime}} \breve{\psi}_{P, \sigma}\right\rangle .
\end{aligned}
$$

In the first step of this computation we noted that when $\partial_{i^{\prime}}$ acts on the factor $\left(P-P_{\mathrm{f}}\right)_{i}$ in $(5.25)$, the resulting integrand has a (trivial) pole of the second order with vanishing residuum. In the second step we inserted twice the decomposition $1=\bar{Q}_{P, \sigma}+\bar{Q}_{P, \sigma}^{\perp}$, namely next to the two integral signs in (5.27). The $\left(\bar{Q}_{P, \sigma}^{\perp}, \bar{Q}_{P, \sigma}^{\perp}\right)$-term vanishes since the corresponding $w$-integrand is holomorphic inside $\gamma_{P, \sigma}$. The $\left(\bar{Q}_{P, \sigma}^{\perp}, \bar{Q}_{P, \sigma}\right)$-term vanishes since the corresponding $\mu$-integrand has a (trivial) pole of second order with vanishing residuum. The $\left(\bar{Q}_{P, \sigma}, \bar{Q}_{P, \sigma}\right)$-term vanishes, since both the $w$ - and $\mu$-integrands have (trivial) poles of the second order with vanishing residuum. The remaining $\left(\bar{Q}_{P, \sigma}, \bar{Q}_{P, \sigma}^{\perp}\right)$-term was evaluated using the Cauchy integral formula and is stated in (5.29).

Next, using the identity

$$
W_{P, \sigma}(-)\left(P-P_{\mathrm{f}}\right) W_{P, \sigma}^{*}=\Gamma_{P, \sigma}-\nabla E_{P, \sigma}
$$

we rearrange formulas (5.25) and (5.28)-(5.29). First, we write

$$
\begin{aligned}
W_{P, \sigma} \partial_{i} \check{\psi}_{P, \sigma} & =W_{P, \sigma} \oint_{\gamma_{P, \sigma}} d w \frac{1}{H_{P, \sigma}-w}(-)\left(P-P_{\mathrm{f}}\right)_{i} \frac{1}{H_{P, \sigma}-w} \breve{\psi}_{P, \sigma} \\
& =\oint_{\gamma_{P, \sigma}} d w \frac{1}{H_{P, \sigma}^{W}-w}\left(\Gamma_{P, \sigma}-\nabla E_{P}\right)_{i} \frac{1}{H_{P, \sigma}^{W}-w} \check{\phi}_{P, \sigma} \\
& =\frac{1}{H_{P, \sigma}^{W}-E_{P, \sigma}}\left(\Gamma_{P, \sigma}\right)_{i} \check{\phi}_{P, \sigma},
\end{aligned}
$$

where the term proportional to $\nabla E_{P, \sigma}$ gave a pole of second order with vanishing residuum, and to the term proportional to $\Gamma_{P, \sigma}$ we applied the Cauchy integral formula. It was important here that $\Gamma_{P, \sigma} \breve{\phi}_{P, \sigma}$ is in the range of $Q_{P, \sigma}^{\perp}$, to ensure the required holomorphy. Second, we compute

$$
\begin{aligned}
W_{P, \sigma} \partial_{i^{\prime}} \partial_{i} \check{\psi}_{P, \sigma}= & \left(\oint_{\gamma_{P, \sigma}} d w \frac{1}{H_{P, \sigma}^{W}-w}\left(\Gamma_{P, \sigma}-\nabla E_{P, \sigma}\right)_{i^{\prime}} \frac{1}{H_{P, \sigma}^{W}-w}\left(\Gamma_{P, \sigma}-\nabla E_{P, \sigma}\right)_{i} \frac{1}{H_{P, \sigma}^{W}-w} \check{\phi}_{P, \sigma}+\left\{i \leftrightarrow i^{\prime}\right\}\right) \\
& +\check{\phi}_{P, \sigma}\left\langle\check{\phi}_{P, \sigma},\left(\Gamma_{P, \sigma}-\nabla E_{P, \sigma}\right)_{i} \frac{\bar{Q}_{P, \sigma}^{\perp}}{\left(H_{P, \sigma}-E_{P, \sigma}\right)^{2}}\left(\Gamma_{P, \sigma}-\nabla E_{P, \sigma}\right)_{i^{\prime}} \check{\phi}_{P, \sigma}\right\rangle \\
= & \left(\frac{Q_{P, \sigma}^{\perp}}{H_{P, \sigma}^{W}-E_{P, \sigma}}\left(\Gamma_{P, \sigma}\right)_{i^{\prime}} \frac{1}{H_{P, \sigma}^{W}-E_{P, \sigma}}\left(\Gamma_{P, \sigma}\right)_{i} \check{\phi}_{P, \sigma}+\left\{i \leftrightarrow i^{\prime}\right\}\right) \\
& -\check{\phi}_{P, \sigma}\left\langle\check{\phi}_{P, \sigma},\left(\Gamma_{P, \sigma}\right)_{i^{\prime}} \frac{1}{\left(H_{P, \sigma}^{W}-E_{P, \sigma}\right)^{2}}\left(\Gamma_{P, \sigma}\right)_{i} \check{\phi}_{P, \sigma}\right\rangle
\end{aligned}
$$

Let us justify this computation: Making use of the fact that $\left\langle\check{\phi}_{P, \sigma},\left(\Gamma_{P, \sigma}\right)_{i} \check{\phi}_{P, \sigma}\right\rangle=0$, we can write

$$
(5.35)=\check{\phi}_{P, \sigma}\left\langle\check{\phi}_{P, \sigma},\left(\Gamma_{P, \sigma}\right)_{i} \frac{1}{\left(H_{P, \sigma}-E_{P, \sigma}\right)^{2}}\left(\Gamma_{P, \sigma}\right)_{i^{\prime}} \check{\phi}_{P, \sigma}\right\rangle,
$$


and we note the sign and $\left(i \leftrightarrow i^{\prime}\right)$ difference w.r.t. to (5.37). Let us now discuss (5.34): The $\left(\nabla E_{P, \sigma}, \nabla E_{P, \sigma}\right)-$ terms vanish as (trivial) poles of the third order with vanishing residua. Thus we can write

$$
\begin{aligned}
(5.34)= & \left(\oint_{\gamma_{P, \sigma}} d w \frac{1}{H_{P, \sigma}^{W}-w}\left(\Gamma_{P, \sigma}\right)_{i^{\prime}} \frac{1}{H_{P, \sigma}^{W}-w}\left(\Gamma_{P, \sigma}\right)_{i} \frac{1}{H_{P, \sigma}^{W}-w} \check{\phi}_{P, \sigma}+\left\{i \leftrightarrow i^{\prime}\right\}\right) \\
& +\left(\oint_{\gamma_{P, \sigma}} d w \frac{1}{H_{P, \sigma}^{W}-w}\left(\Gamma_{P, \sigma}\right)_{i^{\prime}} \frac{1}{H_{P, \sigma}^{W}-w}\left(-\nabla E_{P, \sigma}\right)_{i} \frac{1}{H_{P, \sigma}^{W}-w} \check{\phi}_{P, \sigma}+\left\{i \leftrightarrow i^{\prime}\right\}\right) \\
& +\left(\oint_{\gamma_{P, \sigma}} d w \frac{1}{H_{P, \sigma}^{W}-w}\left(-\nabla E_{P, \sigma}\right)_{i^{\prime}} \frac{1}{H_{P, \sigma}^{W}-w}\left(\Gamma_{P, \sigma}\right)_{i} \frac{1}{H_{P, \sigma}^{W}-w} \check{\phi}_{P, \sigma}+\left\{i \leftrightarrow i^{\prime}\right\}\right) .
\end{aligned}
$$

Now we note that $(5.40)+(5.41)=0$. Indeed, by combining the explicitly stated term in (5.40) with the $\left\{i \leftrightarrow i^{\prime}\right\}$ term in (5.41) (and vice versa) we obtain

$$
(5.40)+(5.41)=\left(-\nabla E_{P, \sigma}\right)_{i} \oint_{\gamma_{P, \sigma}} d w \frac{d}{d w}\left(\frac{1}{H_{P, \sigma}^{W}-w}\left(\Gamma_{P, \sigma}\right)_{i^{\prime}} \frac{1}{H_{P, \sigma}^{W}-w}\right) \check{\phi}_{P, \sigma}+\left\{i \leftrightarrow i^{\prime}\right\}=0,
$$

where in the last step we used the fact that the derivative of a meromorphic function w.r.t. $w$ can only have a vanishing residuum, which follows by inspection of the Laurent series. Given this, we can come back to (5.39) and compute

$$
\begin{aligned}
\text { (5.34) }= & \left(\oint_{\gamma_{P, \sigma}} d w \frac{1}{E_{P, \sigma}-w} \frac{Q_{P, \sigma}^{\perp}}{H_{P, \sigma}^{W}-w}\left(\Gamma_{P, \sigma}\right)_{i^{\prime}} \frac{1}{H_{P, \sigma}^{W}-w}\left(\Gamma_{P, \sigma}\right)_{i} \check{\phi}_{P, \sigma}+\left\{i \leftrightarrow i^{\prime}\right\}\right) \\
& +\left(\oint_{\gamma_{P, \sigma}} d w \frac{1}{\left(E_{P, \sigma}-w\right)^{2}} \check{\phi}_{P, \sigma}\left\langle\check{\phi}_{P, \sigma},\left(\Gamma_{P, \sigma}\right)_{i^{\prime}} \frac{1}{H_{P, \sigma}^{W}-w}\left(\Gamma_{P, \sigma}\right)_{i} \check{\phi}_{P, \sigma}\right\rangle+\left\{i \leftrightarrow i^{\prime}\right\}\right) \\
= & \left(\frac{Q_{P, \sigma}^{\perp}}{H_{P, \sigma}^{W}-E_{P, \sigma}}\left(\Gamma_{P, \sigma}\right)_{i^{\prime}} \frac{1}{H_{P, \sigma}^{W}-E_{P, \sigma}}\left(\Gamma_{P, \sigma}\right)_{i} \check{\phi}_{P, \sigma}+\left\{i \leftrightarrow i^{\prime}\right\}\right) \\
& -\left(\check{\phi}_{P, \sigma}\left\langle\check{\phi}_{P, \sigma},\left(\Gamma_{P, \sigma}\right)_{i^{\prime}} \frac{1}{\left(H_{P, \sigma}^{W}-E_{P, \sigma}\right)^{2}}\left(\Gamma_{P, \sigma}\right)_{i} \check{\phi}_{P, \sigma}\right\rangle+\left\{i \leftrightarrow i^{\prime}\right\}\right),
\end{aligned}
$$

where we applied the Cauchy integral formula to (5.43) and computed the residuum of a meromorphic function with a (non-trivial) pole of second order in (5.44). We remark that (5.46) can alternatively be obtained from the Cauchy formula and

$$
\oint_{\gamma_{P, \sigma}} d w \frac{d}{d w}\left(\frac{1}{\left(E_{P, \sigma}-w\right)} \check{\phi}_{P, \sigma}\left\langle\check{\phi}_{P, \sigma},\left(\Gamma_{P, \sigma}\right)_{i^{\prime}} \frac{1}{H_{P, \sigma}^{W}-w}\left(\Gamma_{P, \sigma}\right)_{i} \check{\phi}_{P, \sigma}\right\rangle\right)=0 .
$$

Making use of (5.45), (5.46), (5.38) we justify the computation (5.34)-(5.37).

Now we proceed to the derivatives of the ground state energy up to third degree, starting from the identity

$$
\partial_{i} E_{P, \sigma}=\left\langle\check{\psi}_{P, \sigma},\left(P-P_{\mathrm{f}}\right)_{i} \check{\psi}_{P, \sigma}\right\rangle .
$$

We first compute

$$
\begin{aligned}
\partial_{i^{\prime}} \partial_{i} E_{P, \sigma} & =\delta_{i, i^{\prime}}+2\left\langle\partial_{i^{\prime}} \check{\psi}_{P, \sigma},\left(P-P_{\mathrm{f}}\right)_{i} \breve{\psi}_{P, \sigma}\right\rangle \\
& =\delta_{i, i^{\prime}}-2\left\langle W_{P, \sigma} \partial_{i^{\prime}} \breve{\psi}_{P, \sigma},\left(\Gamma_{P, \sigma}-\nabla E_{P, \sigma}\right)_{i} \check{\phi}_{P, \sigma}\right\rangle \\
& =\delta_{i, i^{\prime}}-2\left\langle\check{\phi}_{P, \sigma},\left(\Gamma_{P, \sigma}\right)_{i^{\prime}} \frac{1}{H_{P, \sigma}^{W}-E_{P, \sigma}}\left(\Gamma_{P, \sigma}\right)_{i} \check{\phi}_{P, \sigma}\right\rangle,
\end{aligned}
$$


where in the first step we used that $J \breve{\psi}_{P, \sigma}=\breve{\psi}_{P, \sigma}$, in the second step we applied (5.30) and in the last step we substituted (5.33) and used that $\left\langle\check{\phi}_{P, \sigma}, \Gamma_{P, \sigma} \check{\phi}_{P, \sigma}\right\rangle=0$.

Next, coming back to (5.49) and using $\left\langle\partial_{i^{\prime}} \breve{\psi}_{P, \sigma}, \breve{\psi}_{P, \sigma}\right\rangle=0$ and (5.30), we obtain

$$
\begin{aligned}
\partial_{i^{\prime \prime}} \partial_{i^{\prime}} \partial_{i} E_{P, \sigma}= & 2\left\langle\partial_{i^{\prime \prime}} \partial_{i^{\prime}} \breve{\psi}_{P, \sigma},\left(P-P_{\mathrm{f}}\right)_{i} \breve{\psi}_{P, \sigma}\right\rangle \\
& +2\left\langle\partial_{i^{\prime}} \check{\psi}_{P, \sigma},\left(P-P_{\mathrm{f}}\right)_{i} \partial_{i^{\prime \prime}} \breve{\psi}_{P, \sigma}\right\rangle \\
= & -2\left\langle W_{P, \sigma} \partial_{i^{\prime \prime}} \partial_{i^{\prime}} \breve{\psi}_{P, \sigma},\left(\Gamma_{P, \sigma}-\nabla E_{P, \sigma}\right)_{i} \check{\phi}_{P, \sigma}\right\rangle \\
& -2\left\langle W_{P, \sigma} \partial_{i^{\prime}} \breve{\psi}_{P, \sigma},\left(\Gamma_{P, \sigma}-\nabla E_{P, \sigma}\right)_{i} W_{P, \sigma} \partial_{i^{\prime \prime}} \check{\psi}_{P, \sigma}\right\rangle .
\end{aligned}
$$

Now making use of (5.33), we can write

$$
(5.53)=-2\left\langle\frac{1}{H_{P, \sigma}^{W}-E_{P, \sigma}}\left(\Gamma_{P, \sigma}\right)_{i^{\prime}} \check{\phi}_{P, \sigma},\left(\Gamma_{P, \sigma}-\nabla E_{P, \sigma}\right)_{i} \frac{1}{H_{P, \sigma}^{W}-E_{P, \sigma}}\left(\Gamma_{P, \sigma}\right)_{i^{\prime \prime}} \check{\phi}_{P, \sigma}\right\rangle .
$$

Furthermore, exploiting (5.36)-(5.37) we obtain

$$
\begin{aligned}
(5.52)= & -2\left(\left\langle\frac{Q_{P, \sigma}^{\perp}}{H_{P, \sigma}^{W}-E_{P, \sigma}}\left(\Gamma_{P, \sigma}\right)_{i^{\prime \prime}} \frac{1}{H_{P, \sigma}^{W}-E_{P, \sigma}}\left(\Gamma_{P, \sigma}\right)_{i^{\prime}} \check{\phi}_{P, \sigma},\left(\Gamma_{P, \sigma}\right)_{i} \check{\phi}_{P, \sigma}\right\rangle+\left\{i^{\prime} \leftrightarrow i^{\prime \prime}\right\}\right) \\
& -2\left(\nabla E_{P, \sigma}\right)_{i}\left\langle\check{\phi}_{P, \sigma},\left(\Gamma_{P, \sigma}\right)_{i^{\prime \prime}} \frac{1}{\left(H_{P, \sigma}^{W}-E_{P, \sigma}\right)^{2}}\left(\Gamma_{P, \sigma}\right)_{i^{\prime}} \check{\phi}_{P, \sigma}\right\rangle .
\end{aligned}
$$

Noting that $\left(\Gamma_{P, \sigma}\right)_{i^{\prime}} \breve{\phi}_{P, \sigma}=J\left(\Gamma_{P, \sigma}\right)_{i^{\prime}} \breve{\phi}_{P, \sigma}$, we can swop the indices $i^{\prime}, i^{\prime \prime}$ in (5.55), which then cancels with the part of (5.54) proportional to $\left(\nabla E_{P, \sigma}\right)_{i}$. Thus, altogether,

$$
\begin{aligned}
\partial_{i^{\prime \prime}} \partial_{i^{\prime}} \partial_{i} E_{P, \sigma}= & -2\left(\left\langle\frac{Q_{P, \sigma}^{\perp}}{H_{P, \sigma}^{W}-E_{P, \sigma}}\left(\Gamma_{P, \sigma}\right)_{i^{\prime \prime}} \frac{1}{H_{P, \sigma}^{W}-E_{P, \sigma}}\left(\Gamma_{P, \sigma}\right)_{i^{\prime}} \check{\phi}_{P, \sigma},\left(\Gamma_{P, \sigma}\right)_{i} \check{\phi}_{P, \sigma}\right\rangle+\left\{i^{\prime} \leftrightarrow i^{\prime \prime}\right\}\right) \\
& -2\left\langle\frac{1}{H_{P, \sigma}^{W}-E_{P, \sigma}}\left(\Gamma_{P, \sigma}\right)_{i^{\prime}} \check{\phi}_{P, \sigma},\left(\Gamma_{P, \sigma}\right)_{i} \frac{1}{H_{P, \sigma}^{W}-E_{P, \sigma}}\left(\Gamma_{P, \sigma}\right)_{i^{\prime \prime}} \check{\phi}_{P, \sigma}\right\rangle \\
= & -\left(\left\langle\check{\phi}_{P, \sigma},\left(\Gamma_{P, \sigma}\right)_{i} \frac{1}{H_{P, \sigma}^{W}-E_{P, \sigma}}\left(\Gamma_{P, \sigma}\right)_{i^{\prime}} \frac{1}{H_{P, \sigma}^{W}-E_{P, \sigma}}\left(\Gamma_{P, \sigma}\right)_{i^{\prime \prime}} \check{\phi}_{P, \sigma}\right\rangle+\{\text { perm }\}\right),
\end{aligned}
$$

where \{perm\} denotes all the remaining permutations of the $i, i^{\prime}, i^{\prime \prime}$ indices and in the last step we made use again of $\left(\Gamma_{P, \sigma}\right)_{i^{\prime}} \check{\phi}_{P, \sigma}=J\left(\Gamma_{P, \sigma}\right)_{i^{\prime}} \check{\phi}_{P, \sigma}$ and of the fact that $\left\langle\check{\phi}_{P, \sigma}, \Gamma_{P, \sigma} \check{\phi}_{P, \sigma}\right\rangle=0$.

Making use of the formulas above and Corollary 5.7, we immediately obtain

$$
\left|\partial_{i} E_{P, \sigma}\right| \leq c, \quad\left|\partial_{i^{\prime \prime}} \partial_{i^{\prime}} \partial_{i} E_{P, \sigma}\right|,\left\|\partial_{i} \breve{\psi}_{P, \sigma}\right\|,\left\|\partial_{i^{\prime}} \partial_{i} \breve{\psi}_{P, \sigma}\right\| \leq c / \sigma^{\delta_{\lambda_{0}}},
$$

The remaining estimate $\left|\partial_{i^{\prime}} \partial_{i} E_{P, \sigma}\right| \leq c$ from Theorem 2.1 (a) and the convexity statement are proven in Appendix E.

\section{A Proof of Proposition 3.2}

In the proof of Proposition 3.2, which is contained in Lemmas A.5, A.9, A.10, A.12, A.14, and formula (A.26) below, we follow mostly [6], but most ideas date back to [29]. First, we define

$$
\begin{aligned}
\left.\Phi\right|_{n} ^{m} & :=\int_{\sigma_{n}}^{\sigma_{m}} d^{3} k \frac{1}{\sqrt{2|k|}}\left\{b(k)+b^{*}(k)\right\}, \\
\left.H_{\mathrm{f}}\right|_{n} ^{m} & :=\int_{\sigma_{n} \leq k \mid \leq \sigma_{m}} d^{3} k|k| b^{*}(k) b(k), \\
\left.P_{\mathrm{f}}\right|_{n} ^{m} & :=\int_{\sigma_{n} \leq|k| \leq \sigma_{m}} d^{3} k k b^{*}(k) b(k),
\end{aligned}
$$


for $m \leq n$ as operators on (a domain in) $\mathcal{F}$. To analyse these quantities we will often use the energy bounds reported in Lemmata A.1-A.4 below:

Lemma A.1. [6, formula (22)] Let $f \in L^{2}\left(\mathbb{R}^{3}\right)$ be s.t. the integrals on the r.h.s. below are well defined and $\psi \in D\left(H_{P, \text { free }}^{1 / 2}\right)$. Then, for $m \geq n$,

$$
\begin{aligned}
\left\|\int_{\sigma_{n} \leq|k| \leq \sigma_{m}} d^{3} k f(k) b(k) \psi\right\| \leq & \left(\int_{\sigma_{n} \leq|k| \leq \sigma_{m}} d^{3} k\left|\frac{f(k)}{\sqrt{|k|}}\right|^{2}\right)^{1 / 2}\left\|\left(\left.H_{\mathrm{f}}\right|_{n} ^{m}\right)^{1 / 2} \psi\right\|, \\
\left\|\int_{\sigma_{n} \leq k \mid \leq \sigma_{m}} d^{3} k f(k) b^{*}(k) \psi\right\| \leq & \left(\int_{\sigma_{n} \leq|k| \leq \sigma_{m}} d^{3} k\left|\frac{f(k)}{\sqrt{|k|}}\right|^{2}\right)^{1 / 2}\left\|\left(\left.H_{\mathrm{f}}\right|_{n} ^{m}\right)^{1 / 2} \psi\right\| \\
& +\left(\int_{\sigma_{n} \leq|k| \leq \sigma_{m}} d^{3} k|f(k)|^{2}\right)^{1 / 2}\|\psi\| .
\end{aligned}
$$

Next, we fix values of various parameters:

$$
\begin{aligned}
& 0<\epsilon \leq 1 / 2, \quad|\lambda| \leq \epsilon^{2}, \\
& \theta=1 / 12, \quad 0<\xi<1 / 3, \quad C_{\nabla E}^{\lambda}=1 / 3+O(\lambda),
\end{aligned}
$$

where $|O(\lambda)| \leq c|\lambda|$. We demand that

$$
1-\theta-C_{\nabla E}^{\lambda} \geq 2 \xi
$$

We list some preparatory facts.

Lemma A.2. [25, Theorem 8] $E_{0, n} \leq E_{P, n}, \quad P \in \mathbb{R}^{3}$.

Lemma A.3. [6, Lemma 3.2] Let $H_{P \text {,free }}$ be the full free Hamiltonian defined in (2.1). Then there exists a finite constant $c_{a}>0$ s.t. for all $P \in \mathbb{R}^{3}$

$$
\left\langle\psi, H_{P, \text { free }} \psi\right\rangle \leq \frac{1}{1-|\lambda| c_{a}}\left[\left\langle\psi, H_{P, n} \psi\right\rangle+|\lambda| c_{a}\langle\psi, \psi\rangle\right]
$$

for $|\lambda| \leq 1,1 / c_{a}$ and $\psi \in D\left(H_{P, \text { free }}^{1 / 2}\right)$.

Proof. From the identity $H_{P, n}=H_{P, \text { free }}+\left.\lambda \Phi\right|_{n} ^{0}$, where the last term denotes the interaction with cut-off $\sigma_{n}$, and from Lemma A.1, we can write for $\psi \in D\left(H_{P, \text { free }}^{1 / 2}\right)$

$$
\left\langle\psi, H_{P, \text { free }} \psi\right\rangle=\left\langle\psi, H_{P, n} \psi\right\rangle-\left\langle\psi,\left.\lambda \Phi\right|_{n} ^{0} \psi\right\rangle
$$

Furthermore, the energy bounds of Lemma A.1 give

$$
\left|\left\langle\psi,\left.\lambda \Phi\right|_{n} ^{0} \psi\right\rangle\right| \leq|\lambda| c_{a}\left(\left\langle\psi, H_{P, \text { free }} \psi\right\rangle+\langle\psi, \psi\rangle\right)
$$

for some $c_{a}>0$. Altogether $\left\langle\psi, H_{P, \text { free }} \psi\right\rangle \leq\left\langle\psi, H_{P, n} \psi\right\rangle+|\lambda| c_{a}\left(\left\langle\psi, H_{P, \text { free }} \psi\right\rangle+\langle\psi, \psi\rangle\right)$, which gives the claim.

In the following we will assume that $|\lambda| \in\left(0, \lambda_{0}\right]$ for some $0<\lambda_{0} \leq 1 /\left(2 c_{a}\right)$. The value of $\lambda_{0}$ will be tacitly reduced in the course of our discussion but it will remain non-zero.

Lemma A.4. [6, Corollary 5.4] The following inequalities hold true: $-|\lambda| c_{a} \leq E_{P, n} \leq \frac{1}{2} P^{2}, \quad P \in \mathbb{R}^{3}, \quad|\lambda| \in$ $\left(0, \lambda_{0}\right]$. 
Proof. We note that $E_{P, n} \leq\left\langle\Omega, H_{P, n} \Omega\right\rangle \leq P^{2} / 2$. Moreover, Lemma A.3 gives for $\psi \in D\left(H_{P, n}\right) \subset D\left(H_{P, \text { free }}^{1 / 2}\right)$

$$
0 \leq\left(1-|\lambda| c_{a}\right) \inf _{\|\psi\|=1}\left\langle\psi, H_{P, \text { free }} \psi\right\rangle \leq \inf _{\|\psi\|=1}\left[\left\langle\psi, H_{P, n} \psi\right\rangle+|\lambda| c_{a}\langle\psi, \psi\rangle\right]=E_{P, n}+|\lambda| c_{a} \square .
$$

Now we are ready to prove estimate (3.7) of Lemma 3.2.

Lemma A.5. The following inequality holds true: $E_{P-k, n}-E_{P, n} \geq-C_{\nabla E}^{\lambda}|k|, \quad k \in \mathbb{R}^{3}$, for $|P| \leq P_{\max }=$ $1 / 3, \quad|\lambda| \in\left(0, \lambda_{0}\right]$.

Remark A.6. The bound $\left|\nabla E_{P, n}\right| \leq C_{\nabla E}^{\lambda}$ easily follows.

Proof. The argument follows closely [8, Section 6] and [6, Corollary 5.4]. By definition of $E_{P, n}:=\inf \sigma\left(H_{P, n}\right)$,

$$
\begin{aligned}
E_{P-k, n}-E_{P, n} & =\inf _{\|\psi\|=1}\left[\left\langle\psi,\left(H_{P-k, n}-H_{P, n}\right) \psi\right\rangle+\left\langle\psi, H_{P, n} \psi\right\rangle-E_{P, n}\right] \\
& \geq \inf _{\|\psi\|=1}\left[\frac{|k|^{2}}{2}-\left|k \|\left\langle\psi,\left(P-P_{\mathrm{f}}\right) \psi\right\rangle\right|+\left\langle\psi, H_{P, n} \psi\right\rangle-E_{P, n}\right],
\end{aligned}
$$

where $\psi \in D\left(H_{P \text {,free }}^{1 / 2}\right)$. By Lemma A.3 and $\|\psi\|=1$

$$
\left|\left\langle\psi,\left(P-P_{\mathrm{f}}\right) \psi\right\rangle\right|^{2} \leq\left\langle\psi,\left(P-P_{\mathrm{f}}\right)^{2} \psi\right\rangle \leq 2\left\langle\psi, H_{P, \text { free }} \psi\right\rangle \leq \frac{2}{1-|\lambda| c_{a}}\left[\left\langle\psi, H_{P, n} \psi\right\rangle+|\lambda| c_{a}\langle\psi, \psi\rangle\right] .
$$

From (A.14) and (A.13) we get

$$
\begin{aligned}
E_{P-k, n}-E_{P, n} & \geq \inf _{\|\psi\|=1}\left[\frac{|k|^{2}}{2}-|k| \frac{\sqrt{2}}{\sqrt{1-|\lambda| c_{a}}} \sqrt{\left\langle\psi, H_{P, n} \psi\right\rangle+|\lambda| c_{a}}+\left\langle\psi, H_{P, n} \psi\right\rangle-E_{P, n}\right] \\
& \geq \inf _{\Lambda \geq 0}\left[\frac{|k|^{2}}{2}-|k| \frac{\sqrt{2}}{\sqrt{1-|\lambda| c_{a}}} \sqrt{\Lambda+E_{P, n}+|\lambda| c_{a}}+\Lambda\right]=: \inf _{\Lambda \geq 0} f(\Lambda) .
\end{aligned}
$$

The infimum is either achieved at $\Lambda^{*}=0$ or at the point where the derivative of $f$ vanishes, i.e.,

$$
\Lambda^{*}=\frac{|k|^{2}}{4} \frac{2}{1-|\lambda| c_{a}}-\left(E_{P, n}+|\lambda| c_{a}\right)
$$

$\Lambda$ is by construction a nonnegative quantity. First we consider the case $\Lambda^{*}=0$. We have

$$
f(0) \geq-|k| \frac{\sqrt{2}}{\sqrt{1-|\lambda| c_{a}}} \sqrt{E_{P, n}+|\lambda| c_{a}} .
$$

By Lemma A.4, we get

$$
E_{P, n}+|\lambda| c_{a} \leq \frac{P^{2}}{2}+|\lambda| c_{a} \leq \frac{P_{\max }^{2}}{2}+|\lambda| c_{a}=\frac{1}{2}\left(\frac{1}{3}\right)^{2}+|\lambda| c_{a}
$$

Therefore

$$
f(0) \geq-|k| P_{\max }(1+O(\lambda)) .
$$

Now we look at the case of $\Lambda^{*}>0$. We have

$$
f\left(\Lambda^{*}\right)=\frac{k^{2}}{2}\left(1-\frac{1}{1-|\lambda| c_{a}}\right)-\left(E_{P, n}+|\lambda| c_{a}\right) .
$$


We proceed as in [8]: It is clear from Lemma A.4 that $E_{P, n}+|\lambda| c_{a} \geq 0$. The constraint $\Lambda^{*}>0$ can be fulfilled only if (see (A.16))

$$
\sqrt{E_{P, n}+|\lambda| c_{a}}<\frac{|k|}{\sqrt{2}}(1+c|\lambda|)
$$

By combining (A.21) with (A.18), we have

$$
E_{P, n}+|\lambda| c_{a}<\frac{|k|}{\sqrt{2}}(1+c|\lambda|) \sqrt{E_{P, n}+|\lambda| c_{a}} \leq \frac{|k|}{\sqrt{2}}(1+c|\lambda|) \sqrt{\frac{1}{2}\left(\frac{1}{3}\right)^{2}+|\lambda| c_{a}} \leq \frac{|k|}{3}\left(1+c^{\prime}|\lambda|\right) .
$$

Thus we get from (A.20) that for $|k| \leq 1$,

$$
f\left(\Lambda^{*}\right) \geq-k^{2} c|\lambda|-\frac{|k|}{3}\left(1+c^{\prime}|\lambda|\right) \geq-\frac{|k|}{3}\left(1+c^{\prime \prime}|\lambda|\right) .
$$

Recall that $P_{\max }=1 / 3$. Therefore, using (A.19), we have altogether for $|k| \leq 1$

$$
E_{P-k, n}-E_{P, n} \geq-C_{\nabla E}^{\lambda}|k|
$$

for $C_{\nabla E}^{\lambda}=1 / 3+O(\lambda)$. Now we consider the case $|k| \geq 1$. We have by Lemma A.2

$$
\begin{array}{r}
E_{P-k, n}-E_{P, n}=\left(E_{P-k, n}-E_{0, n}\right)+\left(E_{0, n}-E_{P, n}\right) \geq\left(E_{0, n}-E_{P, n}\right) \\
\geq-C_{\nabla E}^{\lambda}\left|P_{\max }\right| \geq-C_{\nabla E}^{\lambda}|k|,
\end{array}
$$

where in the second line we used (A.24) in the case $k=P$.

The following statement is similar to [6, Lemma 5.5], except that we introduce an additional parameter $\eta$ in order to suitably adjust our constants.

Lemma A.7. Let $|\lambda| \in\left(0, \lambda_{0}\right]$ and $|P| \leq P_{\max }=1 / 3$. Assume that for some $7 / 4>\eta \geq 1$ :

1. $E_{P, n-1}$ is the non-degenerate eigenvalue of $H_{P, n-1} 1 \mathcal{F}_{n-1}$ with eigenvector $\psi_{P, n-1}$.

2. $\operatorname{Gap}\left(H_{P, n-1} 1 \mathcal{F}_{n-1}\right) \geq \eta \xi \sigma_{n-1}$.

This implies that $E_{P, n-1}$ is also the non-degenerate ground state energy of $H_{P, n-1} 1 \mathcal{F}_{n}$ with eigenvector $\psi_{P, n-1} \otimes$ $\Omega$. Furthermore,

$$
\operatorname{Gap}\left(H_{P, n-1} 1 \mathcal{F}_{n}\right) \geq \inf _{\mathcal{F}_{n} \ni \psi \perp \psi_{P, n-1} \otimes \Omega}\left\langle H_{P, n-1}-\left.1 \otimes \theta H_{\mathrm{f}}\right|_{n} ^{n-1}-E_{P, n-1}\right\rangle_{\psi} \geq 2 \xi \sigma_{n},
$$

where $\langle\cdot\rangle_{\psi}:=\frac{\langle\psi, \cdot \psi\rangle}{\langle\psi, \psi\rangle}$ and the infimum is taken over non-zero $\psi \in D\left(H_{P, \text { free }}\right)$.

Remark A.8. From (A.34) below and from Lemma A.5, it can be concluded that for $\left(1-\theta-C_{\nabla E}^{\lambda}\right) \geq 0$

$$
\inf _{\mathcal{F}_{n} \ni \psi \perp \psi_{P, n-1} \otimes \Omega}\left\langle H_{P, n-1}-\left.1 \otimes \theta H_{\mathrm{f}}\right|_{n} ^{n-1}-E_{P, n-1}\right\rangle_{\psi} \geq 0
$$

without assuming 1., 2..

Proof. Using 1. a computation gives $H_{P, n-1}\left(\psi_{P, n-1} \otimes \Omega\right)=E_{P, n-1}\left(\psi_{P, n-1} \otimes \Omega\right)$, since the interaction (but not $H_{\mathrm{f}}$, $P_{\mathrm{f}}$ inside of $\left.H_{P, n-1}\right)$ is cut-off at $\sigma_{n-1}$. To prove the simplicity of the eigenvalue, we write

$$
\inf _{\mathcal{F}_{n} \ni \varphi \perp \psi_{P, n-1} \otimes \Omega}\left\langle H_{P, n-1}-E_{P, n-1}\right\rangle_{\varphi} \geq \inf _{\mathcal{F}_{n} \ni \varphi \perp \psi_{P, n-1} \otimes \Omega}\left\langle H_{P, n-1}-\left.1 \otimes \theta H_{\mathrm{f}}\right|_{n} ^{n-1}-E_{P, n-1}\right\rangle_{\varphi}
$$


which is the first inequality in (A.26). Set $P_{\psi}:=\left(\left|\psi_{P, n-1}\right\rangle\left\langle\psi_{P, n-1}\right|\right) /\left\|\psi_{P, n-1}\right\|^{2}$ and $P_{0}:=|\Omega\rangle\langle\Omega|$. Note that $X:=\left(P_{\psi} \otimes P_{0}\right)^{\perp} \mathcal{F}_{n}$ is an invariant subspace of the operator $A:=H_{P, n-1}-\left.1 \otimes \theta H_{\mathrm{f}}\right|_{n} ^{n-1}-E_{P, n-1}$, thus we can consider this operator on this subspace. Furthermore, $\left(1 \otimes P_{0}\right)$ commutes with $\left(P_{\psi} \otimes P_{0}\right)$ and $A$, so we can write

$$
\text { r.h.s. }(A .28)=\inf \sigma(A 1 X)=\min \left(\inf \sigma\left(\left(1 \otimes P_{0}\right) A 1 X\right), \inf \sigma\left(\left(1 \otimes P_{0}\right)^{\perp} A 1 X\right)\right) .
$$

By considering $\left\langle\left(1 \otimes P_{0}\right) A\right\rangle_{\varphi}$ for $\varphi=\sum_{i, i^{\prime}} c_{i, i^{\prime}} e_{i}^{(1)} \otimes e_{i^{\prime}}^{(2)}$, where the orthonormal bases $\left\{e_{i}^{(j)}\right\}_{i \in \mathbb{N}_{0}}$ are chosen so that $e_{0}^{(1)}=\psi_{P, n} /\left\|\psi_{P, n}\right\|$ and $e_{0}^{(2)}=\Omega$, it is easy to see that $\inf \sigma\left(\left(1 \otimes P_{0}\right) A 1 X\right)=\operatorname{Gap}\left(H_{P, n-1} 1 \mathcal{F}_{n-1}\right)$. Let us now analyse the second term: Let $N$ be the number operator on $\left.\mathcal{F}\right|_{n} ^{n-1}$ and $P_{m}$ its spectral projections corresponding to eigenvalues $m \in \mathbb{N}_{0}$. Since $1 \otimes P_{m}$ commute with $A$ and $\left(P_{\psi} \otimes P_{0}\right)$, we can write

$$
\inf \sigma\left(\left(1 \otimes P_{0}\right)^{\perp} A 1 X\right)=\inf _{m \geq 1} \inf \sigma\left(\left(1 \otimes P_{m}\right)\left(1 \otimes P_{0}\right)^{\perp} A 1 X\right) \geq \inf _{m \geq 1} \inf \sigma\left(\left(1 \otimes P_{m}\right) A\right) .
$$

Now we can write

$$
\begin{aligned}
& \left(1 \otimes P_{m}\right) A \simeq \int_{K_{m}}^{\oplus} d^{3 m} k\left(P_{m} A\right)(k), \\
& \left(P_{m} A\right)(k):=\frac{1}{2}\left(P-P_{\mathrm{f}}-\sum_{j=1}^{m} k_{j}\right)^{2}+H_{\mathrm{f}}+\left.\lambda \Phi\right|_{n-1} ^{0}+(1-\theta) \sum_{j=1}^{m}\left|k_{j}\right|-E_{P, n-1},
\end{aligned}
$$

where $K_{m}:=\left\{\left(k_{1}, \ldots, k_{m}\right) \in \mathbb{R}^{3 m}\left|\sigma_{n} \leq\right| k_{i} \mid \leq \sigma_{n-1}\right\}$. Consequently,

$$
\inf _{m \geq 1} \inf \sigma\left(\left(1 \otimes P_{m}\right) A\right)=\inf _{m \geq 1} \inf _{k \in K_{m}} \sigma\left(\left(P_{m} A\right)(k)\right)=\inf _{m \geq 1} \inf _{k \in K_{m}} \inf _{\varphi \in \mathcal{F}_{n-1}}\left\langle\left(P_{m} A\right)(k)\right\rangle_{\varphi}
$$

Thus we have

$$
\begin{aligned}
& \inf _{k \in K_{m}} \inf _{\varphi \in \mathcal{F}_{n-1}}\left\langle\left(P_{m} A\right)(k)\right\rangle_{\varphi} \\
& \geq \inf _{k \in K_{m}} \inf _{\varphi \in \mathcal{F}_{n-1}}\left\langle\frac{1}{2}\left(P-P_{\mathrm{f}}-\sum_{j=1}^{m} k_{j}\right)^{2}+H_{\mathrm{f}}+\left.\lambda \Phi\right|_{n-1} ^{0}+(1-\theta) \sum_{j=1}^{m}\left|k_{j}\right|-E_{P, n-1}\right\rangle_{\varphi} \\
& \geq \inf _{k_{j} \in\left[\sigma_{n}, \sigma_{n-1}\right)}\left((1-\theta) \sum_{j=1}^{m}\left|k_{j}\right|+E_{P-\sum_{j=1}^{m} k_{j}, n-1}-E_{P, n-1}\right) .
\end{aligned}
$$

Now we note that by Lemma A.5

$$
E_{P-\sum_{j=1}^{m} k_{j}, n-1}-E_{P, n-1} \geq-C_{\nabla E}^{\lambda} \sum_{i=1}^{m}\left|k_{i}\right| .
$$

Hence, by (A.8),

$$
(A .34) \geq \inf _{k_{j} \in\left[\sigma_{n}, \sigma_{n-1}\right)}\left(\left(1-\theta-C_{\nabla E}^{\lambda}\right) \sum_{j=1}^{m}\left|k_{j}\right|\right) \geq\left(1-\theta-C_{\nabla E}^{\lambda}\right) \sigma_{n} \geq 2 \xi \sigma_{n} .
$$

Summing up,

$$
\inf _{\mathcal{F}_{n} \ni \psi \perp \psi_{P, n-1} \otimes \Omega}\left\langle H_{P, n-1}-E_{P, n-1}\right\rangle_{\psi} \geq \min \left\{\operatorname{Gap}\left(H_{P, n-1} 1 \mathcal{F}_{n-1}\right), 2 \xi \sigma_{n}\right\}=\min \left(\eta \xi \sigma_{n-1}, 2 \xi \sigma_{n}\right) \geq 2 \xi \sigma_{n} .
$$

Here we made use of the fact that $\epsilon \leq 1 / 2$ and $\eta \geq 1$. This also implies that: a) $\psi_{P, n-1} \otimes \Omega$ is the nondegenerate ground state of $H_{P, n-1} 1 \mathcal{F}_{n}$ since we looked at all the vectors orthogonal to it; b) $\operatorname{Gap}\left(H_{P, n-1} 1\right.$ $\left.\mathcal{F}_{n}\right)=\inf _{\mathcal{F}_{n} \ni \psi \perp \psi_{P, n-1} \otimes \Omega}\left\langle H_{P, n-1}-E_{P, n-1}\right\rangle_{\psi} \geq 2 \xi \sigma_{n}$. 
Lemma A.9. [6, Lemma 5.6] For $n \geq 1$ and $|\lambda| \in\left(0, \lambda_{0}\right]$ the assumptions of Lemma A.7 imply that the resolvent

$$
\frac{1}{H_{P, n}-z}
$$

restricted to $\mathcal{F}_{n}$ is well-defined in the domain

$$
\frac{1}{4} \xi \sigma_{n} \leq\left|E_{P, n-1}-z\right| \leq \eta \xi \sigma_{n}
$$

Proof. We recall that $H_{P, n} 1 \mathcal{F}_{n}=H_{P, n-1} 1 \mathcal{F}_{n}+\left.\lambda \Phi\right|_{n} ^{n-1}$. We note that by Lemma A.7 $z$ is in the resolvent set of $H_{P, n-1} 1 \mathcal{F}_{n}$. Thus for $A(z):=H_{P, n-1} 1 \mathcal{F}_{n}-z$ we can define $A(z)^{-1}$ and $A(z)^{-1 / 2}$ (for some choice of the square root). Setting $B:=\left.\lambda \Phi\right|_{n} ^{n-1}$ we can thus define the resolvent in (A.38) by

$$
(A(z)+B)^{-1}:=A(z)^{-1 / 2} \sum_{j=0}^{\infty}\left[A(z)^{-1 / 2} B A(z)^{-1 / 2}\right]^{j} A(z)^{-1 / 2}
$$

provided that

$$
\left\|A(z)^{-1 / 2} B A(z)^{-1 / 2}\right\|_{\mathcal{F}_{n}}<1
$$

within the specified restrictions. We denote by $B^{( \pm)}$the creation and annihilation part of $B$. We have by the energy bounds from Lemma A.1

$$
\begin{aligned}
\left\|B^{(-)} A(z)^{-1 / 2}\right\|_{\mathcal{F}_{n}} & \leq|\lambda| c\left((1-\epsilon) \sigma_{n-1}\right)^{1 / 2}\left\|\left(\left.H_{\mathrm{f}}\right|_{n} ^{n-1}\right)^{1 / 2} A(z)^{-1 / 2}\right\|_{\mathcal{F}_{n}}, \\
\left\|A(z)^{-1 / 2} B^{(+)}\right\|_{\mathcal{F}_{n}} & =\left\|B^{(-)}\left(A(z)^{-1 / 2}\right)^{*}\right\|_{\mathcal{F}_{n}} \leq|\lambda| c\left((1-\epsilon) \sigma_{n-1}\right)^{1 / 2}\left\|\left(\left.H_{\mathrm{f}}\right|_{n} ^{n-1}\right)^{1 / 2}\left(A(z)^{-1 / 2}\right)^{*}\right\|_{\mathcal{F}_{n}},
\end{aligned}
$$

and we have $\left\|\left(\left.H_{\mathrm{f}}\right|_{n} ^{n-1}\right)^{1 / 2}\left(A(z)^{-1 / 2}\right)^{*}\right\|_{\mathcal{F}_{n}}=\left\|\left(\left.H_{\mathrm{f}}\right|_{n} ^{n-1}\right)^{1 / 2}\left(A(z)^{-1 / 2}\right)\right\|_{\mathcal{F}_{n}}$, since $\left.H_{\mathrm{f}}\right|_{n} ^{n-1}$ and $H_{P, n-1} 1 \mathcal{F}_{n}$ commute. By the same token

$$
\begin{aligned}
& \left\|\left(\left.H_{\mathrm{f}}\right|_{n} ^{n-1}\right)^{1 / 2}\left(H_{P, n-1}-z\right)^{-1 / 2}\right\|_{\mathcal{F}_{n}} \\
& =\theta^{-1 / 2}\left\|\left(\left.\theta H_{f}\right|_{n} ^{n-1}\right)^{1 / 2}\left(H_{P, n-1}-\left.\theta H_{\mathrm{f}}\right|_{n} ^{n-1}-E_{P, n-1}+\left.\theta H_{\mathrm{f}}\right|_{n} ^{n-1}+E_{P, n-1}-z\right)^{-1 / 2}\right\|_{\mathcal{F}_{n}} \\
& \leq \theta^{-1 / 2}\left\|\left(\left.\theta H_{\mathrm{f}}\right|_{n} ^{n-1}\right)^{1 / 2}\left(2 \xi \sigma_{n}-\eta \xi \sigma_{n}+\left.\theta H_{\mathrm{f}}\right|_{n} ^{n-1}\right)^{-1 / 2}\right\|_{\mathcal{F}_{n}} \leq \theta^{-1 / 2}
\end{aligned}
$$

where we made use of (A.26) of Lemma A.7 combined with the fact that $\left.\theta H_{\mathrm{f}}\right|_{n} ^{n-1}$ effectively projects onto the orthogonal complement of the ground state of $H_{P, n-1}$ on $\mathcal{F}_{n}$. Now using Lemma A.7, we get

$$
\begin{aligned}
\left\|A(z)^{-1 / 2}\right\|_{\mathcal{F}_{n}}^{2} & =\left\|\left(H_{P, n-1}-E_{P, n-1}+E_{P, n-1}-z\right)^{-1 / 2}\right\|_{\mathcal{F}_{n}}^{2} \\
& \leq \max \left\{\frac{1}{(1 / 4) \xi \sigma_{n}}, \frac{1}{(2-\eta)\left(\xi \sigma_{n}\right)}\right\}=\frac{4}{\xi \sigma_{n}},
\end{aligned}
$$

where $(2-\eta) \xi \sigma_{n}=2 \xi \sigma_{n}-\eta \xi \sigma_{n}$ comes from (A.26) and (A.39) and we note that $(2-\eta)>1 / 4$. Thus altogether,

$$
(A .41) \leq c|\lambda|\left(\frac{\sigma_{n-1}}{\sigma_{n}}\right)^{1 / 2} \leq c|\lambda|^{3 / 4}<1,
$$

since $|\lambda| \leq \epsilon^{2}$ and $\theta=1 / 12$ and the last inequality may require to reduce $\lambda_{0}$.

Now we choose $\eta=4 / 3$ and $\xi=1 / 4$ s.t. $\eta \xi=1 / 3$ and define the contour

$$
\gamma_{P, n}:=\left\{z \in \mathbb{C}|| E_{P, n-1}-z \mid=\frac{1}{3} \sigma_{n}\right\} .
$$


Lemma A.10. [6, Theorem 4.8] For all $n \geq 0$ and $|\lambda| \in\left(0, \lambda_{0}\right]$ the following holds true:

i) $E_{P, n}:=\inf \sigma\left(H_{P, n} 1 \mathcal{F}\right)=\inf \sigma\left(H_{P, n} 1 \mathcal{F}_{n}\right)$ is the non-degenerate ground state energy of $H_{P, n} 1 \mathcal{F}_{n}$.

ii) $\operatorname{Gap}\left(H_{P, n} 1 \mathcal{F}_{n}\right) \geq \eta \xi \sigma_{n}=(1 / 3) \sigma_{n}$.

iii) The vectors $\psi_{P, 0}=\Omega$ and $\psi_{P, n}:=\bar{Q}_{P, n} \psi_{P, n-1}$, where $\bar{Q}_{P, n}: \mathcal{F}_{n} \rightarrow \mathcal{F}_{n}$,

$$
\bar{Q}_{P, n}:=\oint_{\gamma_{P, n}} \frac{d w_{n}}{H_{P, n}-w_{n}}, \quad n \geq 1
$$

are well defined and non-zero. The vector $\psi_{P, n}$ is the unique (up to phase and normalization) ground state of $H_{P, n} 1 \mathcal{F}_{n}$.

Proof. The proof is by induction. Statements i), ii), iii) for $(n-1)$ will be referred to as assumptions a-i), a-ii), a-iii) while the same statements for $n$ as claims c-i), c-ii), c-iii).

For $n=0$ the claims can be verified as follows: We show that $P^{2} / 2$ is an isolated eigenvalue of $H_{P, 0} 1 \mathcal{F}_{0}$ with a gap larger than $(1 / 3) \sigma_{0}=1 / 3$. We have

$$
H_{P, 0}=\frac{1}{2}\left(P-P_{\mathrm{f}}\right)^{2}+H_{\mathrm{f}}
$$

and we consider this operator on $\mathcal{F}_{0}:=\Gamma\left(L^{2}\left(\mathbb{R}^{3} \backslash B_{\sigma_{0}}\right), d^{3} k\right)$. We note that $\Omega$ is an eigenvector and the corresponding eigenvalue is $P^{2} / 2$. The gap on the $m$-particle subspace is determined by the following function of $\left|k_{i}\right| \geq 1$ :

$$
\begin{aligned}
f\left(k_{1}, \ldots, k_{m}\right) & =\frac{1}{2}\left(P-\sum_{i=1}^{m} k_{i}\right)^{2}+\sum_{i=1}^{m}\left|k_{i}\right|-\frac{1}{2} P^{2} \geq-P \cdot \sum_{i=1}^{m} k_{i}+\sum_{i=1}^{m}\left|k_{i}\right| \\
& \geq\left(1-\left|P_{\max }\right|\right) \sum_{i=1}^{m}\left|k_{i}\right| \geq 2 / 3=(2 / 3) \sigma_{0} \geq(1 / 3) \sigma_{0} .
\end{aligned}
$$

Hence $P^{2} / 2=\inf \sigma\left(H_{P, 0} 1 \mathcal{F}_{0}\right)=\inf \sigma\left(H_{P, 0} 1 \mathcal{F}\right)=: E_{P, 0}$ and the gap on $\mathcal{F}_{0}$ is bounded as required.

Now we proceed with the inductive argument:

1. We have by a-i), a-ii) and Lemma A.7

$$
\operatorname{Gap}\left(H_{P, n-1} 1 \mathcal{F}_{n}\right) \geq \frac{1}{2} \sigma_{n}
$$

By Lemma A.9 we get that the resolvent $\left(H_{P, n}-z\right)^{-1} 1 \mathcal{F}_{n}$ is well defined for $(1 / 16) \sigma_{n} \leq\left|E_{P, n-1}-z\right| \leq$ $(1 / 3) \sigma_{n}$. (Since the resolvent set is open, it actually must be well defined in a slightly larger set).

2. Now we use Kato perturbation theory: We note that $H_{P, n} 1 \mathcal{F}_{n}=H_{P, n-1} 1 \mathcal{F}_{n}+\left.\lambda^{*} \Phi\right|_{n} ^{n-1}$ for $\lambda^{*}=\lambda$. We introduce auxiliary Hamiltonians $H_{P, n}^{\lambda^{*}}$ defined as above with $\left|\lambda^{*}\right| \leq|\lambda|$. We note that the corresponding expression for the ground state projection $\bar{Q}_{P, n}^{\lambda^{*}}$ is well-defined for small $\lambda^{*}$ by the analytic perturbation theory and the bound (A.51) on the gap of the 'unperturbed' Hamiltonian. But by Lemma A.9 the formula can be extended to $\lambda^{*}=\lambda$. (Indeed, if some piece of the spectrum would hit the contour of integration as $\left|\lambda^{*}\right|$ increases, the norm of the resolvent would blow up. But this cannot happen by Lemma A.9). Thus Kato theory gives that $E_{P, n}$ is an isolated, simple eigenvalue of $H_{P, n}$. Since $E_{P, n} \leq E_{P, n-1}$ by Lemma A.12 below, we also have that

$$
\operatorname{Gap}\left(H_{P, n} 1 \mathcal{F}_{n}\right) \geq(1 / 3) \sigma_{n},
$$

due to the upper bound in (A.39). 
3. It suffices to show that the vector $\psi_{P, n}=\bar{Q}_{P, n} \psi_{P, n-1}$ is non-zero. We consider the difference

$$
\left\|\psi_{P, n}-\psi_{P, n-1}\right\|=\left\|\bar{Q}_{P, n} \psi_{P, n-1}-\psi_{P, n-1}\right\|
$$

Similarly as in the proof of Lemma A.9 we set $A\left(w_{n}\right):=\left(H_{P, n-1} 1 \mathcal{F}_{n}-w_{n}\right), B:=\left.\lambda \Phi\right|_{n} ^{n-1}$ and write

$$
\bar{Q}_{P, n} \psi_{P, n-1}-\psi_{P, n-1}=\oint_{\gamma_{P, n}} d w_{n} A\left(w_{n}\right)^{-1 / 2} \sum_{j=1}^{\infty}\left(A\left(w_{n}\right)^{-1 / 2} B A\left(w_{n}\right)^{-1 / 2}\right)^{j} A\left(w_{n}\right)^{-1 / 2} \psi_{P, n-1} .
$$

Thus taking into account (A.45), (A.46) from the proof of Lemma A.9 and the length of the integration contour, we obtain

$$
\left\|\psi_{P, n}-\psi_{P, n-1}\right\| \leq c|\lambda|^{3 / 4}\left\|\psi_{P, n-1}\right\|
$$

from which we conclude $\left\|\psi_{P, n}\right\| \geq\left(1-c|\lambda|^{3 / 4}\right)\left\|\psi_{P, n-1}\right\|$.

Remark A.11. We recall that $E_{P, n}:=\inf \sigma\left(H_{P, n} 1 \mathcal{F}\right)$ according to the original definition in (2.6). Since, by construction, the corresponding eigenvector of $H_{P, n} 1 \mathcal{F}$ has the form $\psi_{P, n} \otimes \Omega$, where $\psi_{P, n} \in \mathcal{F}_{n}$, we have $E_{P, n}=\inf \sigma\left(H_{P, n} 1 \mathcal{F}_{n}\right)$. This explains the second equality in Lemma A.10 (i).

In Lemmata A.12 and A.14 we prove the estimates stated in (3.9) and (3.10) of Proposition 3.2. Lemma A.13 is needed for the proof of Lemma A.14.

Lemma A.12. [29, Lemma 1.2] Recall that $E_{P, n-1}:=\inf \sigma\left(H_{P, n-1} 1 \mathcal{F}\right), E_{P, n}:=\inf \sigma\left(H_{P, n} 1 \mathcal{F}\right)$. Suppose that $H_{P, n-1} 1 \mathcal{F}_{n-1}$ has an eigenvector $\psi_{P, n-1}$ and the corresponding eigenvalue coincides with $E_{P, n-1}$. Then $E_{P, n-1} \geq E_{P, n}$

Proof. The statement follows from

$$
E_{P, n-1}=\frac{\left\langle\psi_{P, n-1} \otimes \Omega, H_{P, n} \psi_{P, n-1} \otimes \Omega\right\rangle}{\left\|\psi_{P, n-1} \otimes \Omega\right\|^{2}} \geq E_{P, n} . \square
$$

Lemma A.13. [6, Lemma 5.9] For some $c>0$ the following inequality holds true: $\left.\theta H_{\mathrm{f}}\right|_{n} ^{n-1}+\left.\lambda \Phi\right|_{n} ^{n-1} \geq$ $-\lambda^{2} \frac{c}{\theta} \sigma_{n-1}$.

Proof. The statement follows from the computation

$$
\begin{aligned}
& \int_{\sigma_{n}}^{\sigma_{n-1}} d^{3} k|k| \theta\left(b^{*}(k) b(k)+\frac{\lambda \chi_{\kappa}(k)|k|^{\bar{\alpha}}}{\sqrt{2|k|}|k| \theta} b(k)+\frac{\lambda \chi_{\kappa}(k)|k|^{\bar{\alpha}}}{\sqrt{2|k|}|k| \theta} b^{*}(k)\right) \\
= & \int_{\sigma_{n}}^{\sigma_{n-1}} d^{3} k|k| \theta\left(b^{*}(k)+\frac{\lambda \chi_{\kappa}(k)|k|^{\bar{\alpha}}}{\sqrt{2|k|}|k| \theta}\right)\left(b(k)+\frac{\lambda \chi_{\kappa}(k)|k|^{\bar{\alpha}}}{\sqrt{2|k|}|k| \theta}\right)-\int_{\sigma_{n}}^{\sigma_{n-1}} d^{3} k|k| \theta\left(\frac{\lambda \chi_{\kappa}(k)|k|^{\bar{\alpha}}}{\sqrt{2|k|}|k| \theta}\right)^{2} \\
\geq & -\frac{1}{\theta} \lambda^{2} \int_{\sigma_{n}}^{\sigma_{n-1}} d^{3} k|k|\left(\frac{\chi_{\kappa}(k)|k|^{\bar{\alpha}}}{\sqrt{2|k|}|k|}\right)^{2} \cdot \square
\end{aligned}
$$

Lemma A.14. [29, Lemma 1.2] Let $|P| \leq P_{\max }=1 / 3$ and $|\lambda| \in\left(0, \lambda_{0}\right]$. Then $\left|E_{P, n-1}-E_{P, n}\right| \leq c_{\Delta E} \lambda^{2} \sigma_{n-1}$.

Proof. We have:

$$
E_{P, n}=\inf \sigma\left(H_{P, n-1} 1 \mathcal{F}_{n}+\left.1 \otimes \lambda \Phi\right|_{n} ^{n-1}\right) .
$$

Since by Lemma A.13 $\left.\theta H_{\mathrm{f}}\right|_{n} ^{n-1}+\left.\lambda \Phi\right|_{n} ^{n-1} \geq-\lambda^{2} \frac{c}{\theta} \sigma_{n-1}$, we get

$$
E_{P, n} \geq \inf \sigma\left\{H_{P, n-1} 1 \mathcal{F}_{n}-\left.1 \otimes \theta H_{\mathrm{f}}\right|_{n} ^{n-1}-\lambda^{2} \frac{c}{\theta} \sigma_{n-1}\right\}
$$


Setting $A:=H_{P, n-1} 1 \mathcal{F}_{n}-\left.1 \otimes \theta H_{\mathrm{f}}\right|_{n} ^{n-1}-\lambda^{2} \frac{c}{\theta} \sigma_{n-1}$ and noting that $\psi_{P, n-1} \otimes \Omega$ is an eigenvector of $A$, we can write

$$
E_{P, n} \geq \min \left\{\langle A\rangle_{\psi_{P, n-1} \otimes \Omega}, \inf _{\mathcal{F}_{n} \ni \downarrow \perp \psi_{P, n-1} \otimes \Omega}\langle A\rangle_{\psi}\right\} .
$$

Clearly, $\langle A\rangle_{\psi_{P, n-1} \otimes \Omega}=E_{P, n-1}-\lambda^{2} \frac{c}{\theta} \sigma_{n-1}$. As for the second quantity under the minimum

$$
\inf _{\mathcal{F}_{n} \ni \psi \perp \psi_{P, n-1} \otimes \Omega}\langle A\rangle_{\psi}=\inf _{\mathcal{F}_{n} \ni \psi \perp \psi_{P, n-1} \otimes \Omega}\left\langle H_{P, n-1} 1 \mathcal{F}_{n}-\left.1 \otimes \theta H_{\mathrm{f}}\right|_{n} ^{n-1}-E_{P, n-1}\right\rangle_{\psi}+E_{P, n-1}-\lambda^{2} \frac{c}{\theta} \sigma_{n-1} .
$$

The first term above is positive by (A.27). Thus we get

$$
E_{P, n} \geq E_{P, n-1}-\lambda^{2} \frac{c}{\theta} \sigma_{n-1} \cdot \square
$$

\section{B Proof of Lemma 3.12}

We consider only the case $z_{n+1} \in \tilde{\gamma}_{P, n+1}$ as the case of contours $\gamma_{P, n+1}$ is analogous and simpler. Let us start with some general considerations. Let $q \in\{1 / 2,1\}$ and $X$ be an operator on $\mathcal{F}$ which maps $\mathcal{F}_{n} \rightarrow \mathcal{F}_{m}$ for some finite $m$, s.t.

$$
\left\|\left(i+H_{P, n-1}^{W}\right)^{-q} X\right\|_{\mathcal{F}_{n}}<\infty
$$

We note for future reference that

$$
\left\|Q_{P, n-1} X\right\|_{\mathcal{F}_{n}}=\left|i+E_{P, n-1}\right|^{q}\left\|Q_{P, n-1}\left(i+H_{P, n-1}^{W}\right)^{-q} X\right\|_{\mathcal{F}_{n}} \leq c\left\|\left(i+H_{P, n-1}^{W}\right)^{-q} X\right\|_{\mathcal{F}_{n}}<\infty,
$$

where $Q_{P, n-1}$ was defined in (3.38). For $z$ outside of the spectrum of $H_{P, n-1}^{W+}$ we consider the following expression on $\mathcal{F}_{n}$

$$
r(z):=\left(\frac{1}{H_{P, n-1}^{W+}-z}\right)^{q} X=\left(\frac{1}{E_{P, n-1}^{+}-z}\right)^{q} Q_{P, n-1} X+Q_{P, n-1}^{\perp}\left(\frac{1}{H_{P, n-1}^{W+}-z}\right)^{q}\left(i+H_{P, n-1}^{W}\right)^{q}\left(i+H_{P, n-1}^{W}\right)^{-q} X,
$$

where $E_{P, n-1}^{+}:=E_{P, n-1}+\left.\Delta c_{P}\right|_{n} ^{n-1}$. We call $r_{1}(z)$ and $r_{2}(z)$ the two terms on the r.h.s. of (B.3). Clearly,

$$
\left\|r_{1}\left(z_{n+1}\right)\right\|_{\mathcal{F}_{n}} \leq \frac{c}{\sigma_{n+1}^{q}}\left\|\left(i+H_{P, n-1}^{W}\right)^{-q} X\right\|_{\mathcal{F}_{n}},
$$

since for sufficiently small $\lambda_{0}$ we can ensure (cf. Remark 3.13)

$$
\left|\Delta c_{P}\right|_{n}^{n-1}|,| E_{P, n-1}-E_{P, n} \mid \leq(1 / 20) \sigma_{n+1} .
$$

Furthermore, we estimate

$$
\left\|r_{2}\left(z_{n+1}\right)\right\|_{\mathcal{F}_{n}} \leq\left\|Q_{P, n-1}^{\perp}\left(\frac{1}{H_{P, n-1}^{W+}-z_{n+1}}\right)^{q}\left(i+H_{P, n-1}^{W}\right)^{q}\right\|_{\mathcal{F}_{n}}\left\|\left(i+H_{P, n-1}^{W}\right)^{-q} X\right\|_{\mathcal{F}_{n}} \leq \frac{c}{\sigma_{n+1}^{q}}\left\|\left(i+H_{P, n-1}^{W}\right)^{-q} X\right\|_{\mathcal{F}_{n}},
$$

where we made use of the gap estimate (3.12). Summarizing the above discussion, we have

$$
\begin{aligned}
& \left\|\frac{1}{H_{P, n-1}^{W+}-z_{n+1}} X\right\|_{\mathcal{F}_{n}} \leq \frac{c}{\sigma_{n+1}}\left\|\left(i+H_{P, n-1}^{W}\right)^{-1} X\right\|_{\mathcal{F}_{n}}, \\
& \left\|\left(\frac{1}{H_{P, n-1}^{W+}-z_{n+1}}\right)^{1 / 2} X\right\|_{\mathcal{F}_{n}} \leq \frac{c}{\sigma_{n+1}^{1 / 2}}\left\|\left(i+H_{P, n-1}^{W}\right)^{-1 / 2} X\right\|_{\mathcal{F}_{n}} .
\end{aligned}
$$

These simple-minded estimates will yield some (but not all) bounds from Lemma 3.12. 
Lemma B.1. Under the assumptions of Lemma 3.12

$$
\begin{aligned}
& \left\|\frac{1}{H_{P, n-1}^{W+}-z_{n+1}}\right\|_{\mathcal{F}_{n}} \leq \frac{c_{0}}{\sigma_{n+1}} \\
& \left\|\frac{1}{H_{P, n-1}^{W+}-z_{n+1}}\left(\Gamma_{P, n-1}\right)_{i}\right\|_{\mathcal{F}_{n}} \leq \frac{c_{0}}{\sigma_{n+1}} \\
& \left\|\frac{1}{H_{P, n-1}^{W+}-z_{n+1}}\left(\left.\mathcal{I}\right|_{n} ^{n-1}\right)_{i}\right\|_{\mathcal{F}_{n}} \leq c_{2}|\lambda|^{3 / 2}
\end{aligned}
$$

Proof. The estimate in (B.9) follows from (B.7) with $X=1$. Given (B.7), to verify (B.10), it suffices to check that

$$
\left\|\left(i+H_{P, n-1}^{W}\right)^{-1} \Gamma_{P, n-1}\right\|_{\mathcal{F}_{n}} \leq c
$$

Since $\Gamma_{P, n-1}:=\nabla E_{P, n-1}-W_{P, n-1}\left(P-P_{\mathrm{f}}\right) W_{P, n-1}^{*}$, we have by (3.8)

$$
\left\|\left(i+H_{P, n-1}^{W}\right)^{-1} \Gamma_{P, n-1}\right\|_{\mathcal{F}_{n}} \leq\left\|\left(i+H_{P, n-1}\right)^{-1}\left(P-P_{\mathrm{f}}\right)\right\|_{\mathcal{F}_{n}}+c
$$

Furthermore, by Lemma A.3

$$
\left\|\left(i+H_{P, n-1}\right)^{-1 / 2}\left(P-P_{\mathrm{f}}\right)\right\|_{\mathcal{F}_{n}}^{2} \leq c\left\|\left(i+H_{P, n-1}\right)^{-1 / 2} H_{P, \text { free }}\left(\left(i+H_{P, n-1}\right)^{-1 / 2}\right)^{*}\right\|_{\mathcal{F}_{n}} \leq c^{\prime} .
$$

As for (B.11), we have by definition (3.28) that $\left|\left(\left.\mathcal{I}\right|_{n} ^{n-1}\right)_{i}\right| \leq c|\lambda|^{2} \sigma_{n-1}$, which gives together with (B.9) and $|\lambda| \leq \epsilon^{4}$ the required bound.

Lemma B.2. Under the assumptions of Lemma 3.12

$$
\left\|\left.\frac{1}{H_{P, n-1}^{W+}-z_{n+1}} \mathcal{L}\right|_{n} ^{n-1}\right\|_{\mathcal{F}_{n}} \leq c_{2}|\lambda|^{1 / 2} .
$$

If $\left.\mathcal{L}\right|_{n} ^{n-1}$ is replaced with $\left.\mathcal{L}^{\prime}\right|_{n} ^{n-1}:=\left[H_{\mathrm{f}},\left.\mathcal{L}\right|_{n} ^{n-1}\right]$ or $\left.\stackrel{\circ}{\mathcal{L}}\right|_{n} ^{n-1}=\sum_{j=1}^{3}\left[\left(P_{\mathrm{f}}\right)_{j},\left(\left.\mathcal{L}\right|_{n} ^{n-1}\right)_{j}\right]$ the bounds can be improved to $c_{2}|\lambda|^{1 / 2} \sigma_{n-1}$.

Proof. We set $A:=H_{P, n-1}+\left.\Delta c_{P}\right|_{n} ^{n-1}-\bar{z}_{n+1}$. Since $\left[W_{P, n-1},\left.\mathcal{L}\right|_{n} ^{n-1}\right]=0$, we have

$$
\left\|\left.\frac{1}{H_{P, n-1}^{W+}-z_{n+1}} \mathcal{L}\right|_{n} ^{n-1}\right\|_{\mathcal{F}_{n}}=\left\|\left.\left(A^{-1}\right)^{*} \mathcal{L}\right|_{n} ^{n-1}\right\|_{\mathcal{F}_{n}} .
$$

By the energy bounds (Lemma A.1) and the fact that $A^{-1 / 2}$ and $\left(\left.H_{\mathrm{f}}\right|_{n} ^{n-1}\right)^{1 / 2}$ commute we have

$$
\begin{aligned}
\left\|\left.\left(A^{-1 / 2}\right)^{*} \mathcal{L}\right|_{n} ^{n-1}\right\|_{\mathcal{F}_{n}}=\left\|\left.\mathcal{L}\right|_{n} ^{n-1} A^{-1 / 2}\right\|_{\mathcal{F}_{n}} & \leq|\lambda| c \sigma_{n-1}^{1 / 2}\left\|\left(\left.H_{\mathrm{f}}\right|_{n} ^{n-1}\right)^{1 / 2} A^{-1 / 2}\right\|_{\mathcal{F}_{n}}+|\lambda| c \sigma_{n-1}\left\|A^{-1 / 2}\right\|_{\mathcal{F}_{n}} \\
& \leq|\lambda| c \sigma_{n-1}^{1 / 2}+|\lambda| c \frac{\sigma_{n-1}}{\left(\sigma_{n+1}\right)^{1 / 2}} .
\end{aligned}
$$

In the last step we used (B.9) and the spectral theorem which give $\left\|A^{-1 / 2}\right\|_{\mathcal{F}_{n}} \leq\left\|A^{-1}\right\|_{\mathcal{F}_{n}}^{1 / 2} \leq c\left(\sigma_{n+1}\right)^{-1 / 2}$, and the following computation similar to (A.44)

$$
\begin{aligned}
\left\|\left(\left.H_{\mathrm{f}}\right|_{n} ^{n-1}\right)^{1 / 2} A^{-1 / 2}\right\|_{\mathcal{F}_{n}} & =\left\|\left(\left.H_{\mathrm{f}}\right|_{n} ^{n-1}\right)^{1 / 2}\left(H_{P, n-1}+\left.\Delta c_{P}\right|_{n} ^{n-1}-z_{n+1}\right)^{-1 / 2}\right\|_{\mathcal{F}_{n}}, \\
& \leq \theta^{-1 / 2}\left\|\left(\left.\theta H_{\mathrm{f}}\right|_{n} ^{n-1}\right)^{1 / 2}\left(H_{P, n-1}-\left.\theta H_{\mathrm{f}}\right|_{n} ^{n-1}-E_{P, n-1}+O\left(\sigma_{n+1}\right)+\left.\theta H_{\mathrm{f}}\right|_{n} ^{n-1}-i \operatorname{Im} z_{n+1}\right)^{-1 / 2}\right\|_{\mathcal{F}_{n}} \\
& \leq \theta^{-1 / 2}\left\|\left(\left.\theta H_{\mathrm{f}}\right|_{n} ^{n-1}\right)^{1 / 2}\left(\sigma_{n} / 2+O\left(\sigma_{n+1}\right)+\left.\theta H_{\mathrm{f}}\right|_{n} ^{n-1}-i \operatorname{Im} z_{n+1}\right)^{-1 / 2}\right\|_{\mathcal{F}_{n}} \leq c,
\end{aligned}
$$


where we made use of the fact that $H_{P, n-1}-\left.\theta H_{\mathrm{f}}\right|_{n} ^{n-1}-E_{P, n-1} \geq \sigma_{n} / 2$ on the subspace orthogonal to $\psi_{P, n-1} \otimes \Omega$ (cf. (A.26)). Since $\left|O\left(\sigma_{n+1}\right)\right|:=\sup _{z_{n+1} \in \gamma_{n+1} \cup \tilde{\gamma}_{n+1}}\left|E_{P, n-1}-E_{P, n}+\Delta c_{P}\right|_{n}^{n-1}+E_{P, n}-\operatorname{Re} z_{n+1} \mid \leq \sigma_{n+1} / 2$, we obtain the last estimate in (B.18). Alluding again to the fact that $\left\|A^{-1 / 2}\right\|_{\mathcal{F}_{n}} \leq c\left(\sigma_{n+1}\right)^{-1 / 2}$ and using $|\lambda| \leq \epsilon^{4}$ we conclude the proof of (B.15). The remaining statements in the lemma follow from obvious modifications of the above discussion.

Lemma B.3. Under the assumptions of Lemma 3.12

$$
\left\|\left.\frac{1}{H_{P, n-1}^{W+}-z_{n+1}} \mathcal{L}\right|_{n} ^{n-1} \cdot \Gamma_{P, n-1}\right\|_{\mathcal{F}_{n}} \leq c_{2}|\lambda|^{1 / 2}
$$

Proof. Since $\Gamma_{P, n-1}:=\nabla E_{P, n-1}-W_{P, n-1}\left(P-P_{\mathrm{f}}\right) W_{P, n-1}^{*}$ and $\left[W_{P, n-1},\left.\mathcal{L}\right|_{n} ^{n-1}\right]=0$, we note the following inequality

$$
\begin{aligned}
\left\|\left.\frac{1}{H_{P, n-1}^{W+}-z_{n+1}} \mathcal{L}\right|_{n} ^{n-1} \cdot \Gamma_{P, n-1}\right\|_{\mathcal{F}_{n}} \leq & \left\|\left.\frac{1}{H_{P, n-1}+\left.\Delta c_{P}\right|_{n} ^{n-1}-z_{n+1}} \mathcal{L}\right|_{n} ^{n-1} \cdot\left(P-P_{\mathrm{f}}\right)\right\|_{\mathcal{F}_{n}} \\
& +\left\|\left.\frac{1}{H_{P, n-1}+\left.\Delta c_{P}\right|_{n} ^{n-1}-z_{n+1}} \mathcal{L}\right|_{n} ^{n-1}\right\|_{\mathcal{F}_{n}}\left|\nabla E_{P, n-1}\right| .
\end{aligned}
$$

By Lemma B.2, using $\left[W_{P, n-1},\left.\mathcal{L}\right|_{n} ^{n-1}\right]=0$ one more time, we have $(B .21) \leq c|\lambda|^{1 / 2}$. As for (B.20), we write

$$
\begin{aligned}
(B .20)= & \left\|\left.\frac{1}{H_{P, n-1}+\left.\Delta c_{P}\right|_{n} ^{n-1}-z_{n+1}} \mathcal{L}\right|_{n} ^{n-1}\right\|_{\mathcal{F}_{n}} \\
& +\left\|\left.\frac{1}{H_{P, n-1}+\left.\Delta c_{P}\right|_{n} ^{n-1}-z_{n+1}}\left(P-P_{\mathrm{f}}\right) \cdot \mathcal{L}\right|_{n} ^{n-1}\right\|_{\mathcal{F}_{n}},
\end{aligned}
$$

where $\left.\stackrel{\circ}{\mathcal{L}}\right|_{n} ^{n-1}=-\sum_{j=1}^{3}\left[\left(\left.\mathcal{L}\right|_{n} ^{n-1}\right)_{j},\left(P_{\mathrm{f}}\right)_{j}\right]$. By the last statement in Lemma B.2 and $\left[W_{P, n-1},\left.\mathcal{L}\right|_{n} ^{n-1}\right]=0$, we have that $(B .22) \leq c|\lambda|^{1 / 2} \sigma_{n-1}$. To (B.23) we apply the energy bounds (Lemma A.1). Setting $A:=H_{P, n-1}+\left.\Delta c_{P}\right|_{n} ^{n-1}-\bar{z}_{n+1}$, we have

$$
\begin{aligned}
\left\|\left.\mathcal{L}\right|_{n} ^{n-1} \cdot\left(P-P_{\mathrm{f}}\right) A^{-1}\right\|_{\mathcal{F}_{n}} & =c|\lambda| \sigma_{n-1}^{1 / 2}\left\|\left(\left.H_{\mathrm{f}}\right|_{n} ^{n-1}\right)^{1 / 2}\left(P-P_{\mathrm{f}}\right) A^{-1}\right\|_{\mathcal{F}_{n}}+c|\lambda| \sigma_{n-1}\left\|\left(P-P_{\mathrm{f}}\right) A^{-1}\right\|_{\mathcal{F}_{n}} \\
& \leq c|\lambda|\left(\frac{\sigma_{n-1}^{1 / 2}}{\sigma_{n+1}^{1 / 2}}+\frac{\sigma_{n-1}}{\sigma_{n+1}}\right)=c|\lambda|^{1 / 2} .
\end{aligned}
$$

Here we made use of $\left\|\left(P-P_{\mathrm{f}}\right) A^{-1}\right\|_{\mathcal{F}_{n}} \leq c / \sigma_{n+1}$, which is shown using (B.7) and (B.14). Furthermore, using that $\left[\left.H_{\mathrm{f}}\right|_{n} ^{n-1}, P-P_{\mathrm{f}}\right]=\left[\left.H_{\mathrm{f}}\right|_{n} ^{n-1}, A\right]=0$,

$$
\begin{aligned}
\left\|\left(\left.H_{\mathrm{f}}\right|_{n} ^{n-1}\right)^{1 / 2}\left(P-P_{\mathrm{f}}\right) A^{-1}\right\|_{\mathcal{F}_{n}} & =\left\|\left(P-P_{\mathrm{f}}\right) A^{-1 / 2}\left(\left.H_{\mathrm{f}}\right|_{n} ^{n-1}\right)^{1 / 2} A^{-1 / 2}\right\|_{\mathcal{F}_{n}} \\
& \leq\left\|\left(P-P_{\mathrm{f}}\right) A^{-1 / 2}\right\|_{\mathcal{F}_{n}}\left\|\left(\left.H_{\mathrm{f}}\right|_{n} ^{n-1}\right)^{1 / 2} A^{-1 / 2}\right\|_{\mathcal{F}_{n}} \leq \frac{c}{\left(\sigma_{n+1}\right)^{1 / 2}},
\end{aligned}
$$

where in the last inequality we exploit (B.18) and (B.14) combined with (B.8). This completes the proof.

Lemma B.4. Under the assumptions of Lemma 3.12

$$
\left\|\frac{1}{H_{P, n-1}^{W+}-z_{n+1}}\left(\left.\mathcal{L}\right|_{n} ^{n-1}\right)^{2}\right\|_{\mathcal{F}_{n}} \leq c|\lambda|^{3 / 2} \sigma_{n-1} .
$$


Proof. We set as before $A:=H_{P, n-1}+\left.\Delta c_{P}\right|_{n} ^{n-1}-\bar{z}_{n+1}$, we then use $\left[W_{P, n-1},\left.\mathcal{L}\right|_{n} ^{n-1}\right]=0$ and apply the energy bounds (Lemma A.1):

$$
\left\|\left(\left.\mathcal{L}\right|_{n} ^{n-1}\right)^{2} A^{-1}\right\|_{\mathcal{F}_{n}} \leq c|\lambda| \sigma_{n-1}^{1 / 2}\left\|\left.\left(\left.H_{\mathrm{f}}\right|_{n} ^{n-1}\right)^{1 / 2} \mathcal{L}\right|_{n} ^{n-1} A^{-1}\right\|_{\mathcal{F}_{n}}+c|\lambda| \sigma_{n-1}\left\|\left.\mathcal{L}\right|_{n} ^{n-1} A^{-1}\right\|_{\mathcal{F}_{n}}
$$

We first estimate the second term on the r.h.s. above applying again the energy bounds

$$
\begin{aligned}
c|\lambda| \sigma_{n-1}\left\|\left.\mathcal{L}\right|_{n} ^{n-1} A^{-1}\right\|_{\mathcal{F}_{n}} & \leq c|\lambda|^{2}\left(\sigma_{n-1}\right)^{3 / 2}\left\|\left(\left.H_{\mathrm{f}}\right|_{n} ^{n-1}\right)^{1 / 2} A^{-1}\right\|_{\mathcal{F}_{n}}+c|\lambda|^{2}\left(\sigma_{n-1}\right)^{2}\left\|A^{-1}\right\|_{\mathcal{F}_{n}} \\
& \leq c|\lambda|^{2}\left(\left(\sigma_{n-1}\right)^{3 / 2} \sigma_{n+1}^{-1 / 2}+\left(\sigma_{n-1}\right)^{2} \sigma_{n+1}^{-1}\right) \\
& \leq c|\lambda|^{3 / 2} \sigma_{n-1},
\end{aligned}
$$

where we made use of (B.18), $\left\|A^{-1 / 2}\right\|_{\mathcal{F}_{n}} \leq c\left(\sigma_{n+1}\right)^{-1 / 2}$ and $|\lambda|^{1 / 2} \leq \epsilon^{2}$. Now we consider the first term on the r.h.s. of (B.27):

$$
\begin{aligned}
\left\|\left.\left(\left.H_{\mathrm{f}}\right|_{n} ^{n-1}\right)^{1 / 2} \mathcal{L}\right|_{n} ^{n-1} A^{-1}\right\|_{\mathcal{F}_{n}}^{2} & =\left\|\left.\left.\left.\left(A^{-1}\right)^{*} \mathcal{L}\right|_{n} ^{n-1} H_{\mathrm{f}}\right|_{n} ^{n-1} \mathcal{L}\right|_{n} ^{n-1} A^{-1}\right\|_{\mathcal{F}_{n}} \\
& \leq\left\|\left.\left.\left(A^{-1}\right)^{*} \mathcal{L}\right|_{n} ^{n-1} \mathcal{L}^{\prime}\right|_{n} ^{n-1} A^{-1}\right\|_{\mathcal{F}_{n}}+\left\|\left.\left.\left.\left(A^{-1}\right)^{*} \mathcal{L}\right|_{n} ^{n-1} \mathcal{L}\right|_{n} ^{n-1} H_{\mathrm{f}}\right|_{n} ^{n-1} A^{-1}\right\|_{\mathcal{F}_{n}} \\
& \leq c|\lambda| \sigma_{n-1}+c\left\|\left(A^{-1}\right)^{*}\left(\left.\mathcal{L}\right|_{n} ^{n-1}\right)^{2}\right\|_{\mathcal{F}_{n}},
\end{aligned}
$$

where $\left.\mathcal{L}^{\prime}\right|_{n} ^{n-1}:=\left[H_{\mathrm{f}},\left.\mathcal{L}\right|_{n} ^{n-1}\right]$ and we applied Lemma B.2 and (B.18) in the last step. Thus altogether we get

$$
\left\|\left(\left.\mathcal{L}\right|_{n} ^{n-1}\right)^{2} A^{-1}\right\|_{\mathcal{F}_{n}} \leq c|\lambda| \sigma_{n-1}^{1 / 2}\left(|\lambda| \sigma_{n-1}+\left\|\left(A^{-1}\right)^{*}\left(\left.\mathcal{L}\right|_{n} ^{n-1}\right)^{2}\right\|_{\mathcal{F}_{n}}\right)^{1 / 2}+c|\lambda|^{3 / 2} \sigma_{n-1}
$$

Set $x:=\left\|\left(\left.\mathcal{L}\right|_{n} ^{n-1}\right)^{2} A^{-1}\right\|_{\mathcal{F}_{n}}$ and shift the term $c|\lambda|^{3 / 2} \sigma_{n-1}$ on the 1.h.s. of (B.30). If $x-c|\lambda|^{3 / 2} \sigma_{n-1} \leq 0$ then the proof is complete. Otherwise, we can square both sides of the resulting inequality which implies the following relation

$$
x^{2}-c_{1}|\lambda|^{3 / 2} \sigma_{n-1} x-c_{2}|\lambda|^{3} \sigma_{n-1}^{2} \leq 0,
$$

with $c_{1}, c_{2} \geq 0$. This leads to $x \leq c|\lambda|^{3 / 2} \sigma_{n-1}$.

Lemma B.5. Under the assumptions of Lemma 3.12

$$
\left\|\frac{1}{H_{P, n-1}^{W+}-z_{n+1}}\left(\left.H_{I}^{W}\right|_{n} ^{n-1}\right)_{\text {quad }}\right\|_{\mathscr{F}_{n}} \leq c|\lambda|^{3 / 2} \sigma_{n-1} .
$$

Proof. We recall that $\left(\left.H_{I}^{W}\right|_{n} ^{n-1}\right)_{\text {quad }}=\frac{1}{2}\left(\left.\mathcal{L}\right|_{n} ^{n-1}+\left.\mathcal{I}\right|_{n} ^{n-1}\right)^{2}$. Thus the statement follows from Lemmas B.1, B.2, B.4, and from $\left|\left(\left.\mathcal{I}\right|_{n} ^{n-1}\right)_{i}\right| \leq c|\lambda|^{2} \sigma_{n-1}$.

Lemma B.6. Under the assumptions of Lemma 3.12

$$
\left\|\frac{1}{H_{P, n-1}^{W+}-z_{n+1}} \Delta\left(\left.H_{I}^{W}\right|_{n} ^{n-1}\right)_{\operatorname{mix}}\right\|_{\mathcal{F}_{n}} \leq c|\lambda|^{1 / 2} \sigma_{n-1} .
$$

Proof. We recall that $\Delta\left(\left.H_{I}^{W}\right|_{n} ^{n-1}\right)_{\text {mix }}=\frac{1}{2} \sum_{j=1}^{3}\left[\left(\Gamma_{P, n-1}\right)_{j},\left(\left.\mathcal{L}\right|_{n} ^{n-1}\right)_{j}\right]$. Thus (B.33) corresponds to the second result of Lemma B.2.

Lemma B.7. Under the assumptions of Lemma 3.12

$$
\left\|\frac{1}{H_{P, n-1}^{W+}-z_{n+1}}\left(\left.H_{I}^{W}\right|_{n} ^{n-1}\right)\right\|_{\mathcal{F}_{n}} \leq c|\lambda|^{1 / 2}
$$

Proof. Given that $\left.H_{I}^{W}\right|_{n} ^{n-1}:=\left(\left.\mathcal{L}\right|_{n} ^{n-1}+\left.\mathcal{I}\right|_{n} ^{n-1}\right) \cdot \Gamma_{P, n-1}+\left(\left.H_{I}^{W}\right|_{n} ^{n-1}\right)_{\text {quad }}+\Delta\left(\left.H_{I}^{W}\right|_{n} ^{n-1}\right)_{\text {mix }}$, the statement follows from Lemmas B.1, B.3, B.5, B.6 and $\left|\left(\left.\mathcal{I}\right|_{n} ^{n-1}\right)_{i}\right| \leq c|\lambda|^{2} \sigma_{n-1}$. 


\section{Proof of Theorem 4.1}

First, we check that the hypotheses holds for $n=0$. Statement i) is obvious since $\Gamma_{P, 0} \Omega=0$. As for ii), we have

$$
\hat{\phi}_{P, 1}-\Omega=\oint_{\gamma_{P, 2}} \frac{d w_{2}}{\hat{H}_{P, 1}^{W}-w_{2}} \Omega-\Omega
$$

We use the resolvent expansion (3.25):

$$
\frac{1}{\hat{H}_{P, 1}^{W}-w_{2}} \Omega=\frac{1}{H_{P, 0}^{W}+\left.\Delta c_{P}\right|_{1} ^{0}-w_{2}} \sum_{j=0}^{\infty}\left\{-\left.H_{I}^{W}\right|_{1} ^{0} \frac{1}{H_{P, 0}^{W}+\left.\Delta c_{P}\right|_{1} ^{0}-w_{2}}\right\}^{j} \Omega,
$$

for $w_{2} \in \gamma_{P, 2}$. The $j=0$ term gives zero contribution to (C.1) due to $H_{P, 0}^{W} \Omega=E_{P, 0} \Omega,\left|E_{P, 0}-E_{P, 1}\right| \leq(1 / 20) \sigma_{2}$, $\left.\Delta c_{P}\right|_{1} ^{0}=(1 / 20) \sigma_{2}$ and $\left|w_{2}-E_{P, 1}\right|=\sigma_{2} / 3$. Thus we have

$$
\begin{aligned}
\left\|\hat{\phi}_{P, 1}-\Omega\right\| & =\left\|\oint_{\gamma_{P, 2}} d w_{2} \frac{1}{H_{P, 0}^{W}+\left.\Delta c_{P}\right|_{1} ^{0}-w_{2}} \sum_{j=1}^{\infty}\left\{-\left.H_{I}^{W}\right|_{1} ^{0} \frac{1}{H_{P, 0}^{W}+\left.\Delta c_{P}\right|_{1} ^{0}-w_{2}}\right\}^{j} \Omega\right\| \\
& \leq \sigma_{2} \frac{c_{0}}{\sigma_{2}} c_{2}|\lambda|^{1 / 2} \frac{1}{1-c_{2}|\lambda|^{1 / 2}} \leq|\lambda|^{1 / 4} \sigma_{0}^{1-\delta},
\end{aligned}
$$

where we made use of estimates (3.47) and (3.51) from Lemma 3.12, (3.56) and $\sigma_{0}=1$. Now iii) follows from ii) via the inverse triangle inequality.

Now we proceed to the inductive argument. The claims in i), ii), and iii) are at the $n+1$-th step by definition, and they will be denoted as c-i), c-ii), and c-iii), respectively. At the $n$-th step they will be denoted as assumptions a-i), a-ii), and a-iii), respectively. All operator norms in the proof are meant on $B\left(\mathcal{F}_{n}\right)$ (the bounded operators on $\mathcal{F}_{n}$ ) unless stated otherwise.

Claim-I) (Here we show the implication: $a-i), a-i i), a-i i i) \rightarrow c-i)$ )

We use the unitary operator $\widetilde{W}_{P, n} W_{P, n}^{*}$ to switch from the given expression to the expression with 'hats'

$$
\left\|\frac{1}{H_{P, n}^{W}-z_{n+1}}\left(\Gamma_{P, n}\right)_{i} \phi_{P, n}\right\|=\left\|\widetilde{W}_{P, n} W_{P, n}^{*} \frac{1}{H_{P, n}^{W}-z_{n+1}}\left(\Gamma_{P, n}\right)_{i} \phi_{P, n}\right\|=\left\|\frac{1}{\hat{H}_{P, n}^{W}-z_{n+1}}\left(\hat{\Gamma}_{P, n}\right)_{i} \hat{\phi}_{P, n}\right\| .
$$

In (C.4) we proceed with the full expansion of $\hat{\phi}_{P, n}, \frac{1}{\hat{H}_{P, n}^{W}-z_{n+1}}$, and $\left(\hat{\Gamma}_{P, n}\right)_{i}$, i.e., we study

$$
\begin{array}{r}
\sum_{l=0}^{\infty} \sum_{l^{\prime}=0}^{\infty} \oint_{\gamma_{P, n+1}} d w_{n+1}\left\{\left.\frac{1}{H_{P, n-1}^{W+}-z_{n+1}}(-) H_{I}^{W}\right|_{n} ^{n-1}\right\}^{l} \frac{1}{H_{P, n-1}^{W+}-z_{n+1}}\left(\Gamma_{P, n-1}+\left.\Delta \Gamma_{P}\right|_{n} ^{n-1}\right)_{i} \times \\
\times\left\{\left.\frac{1}{H_{P, n-1}^{W+}-w_{n+1}}(-) H_{I}^{W}\right|_{n} ^{n-1}\right\}^{l^{\prime}} \frac{1}{H_{P, n-1}^{W+}-w_{n+1}} \phi_{P, n-1} .
\end{array}
$$

By splitting $\left.\Delta \Gamma_{P}\right|_{n} ^{n-1}$ into $\left.\Delta^{\prime} \Gamma_{P}\right|_{n} ^{n-1}+\left.\mathcal{I}\right|_{n} ^{n-1}$ (see (3.34) and (3.35)) we can write

$$
\begin{aligned}
(C .5)=\sum_{l=0}^{\infty} \sum_{l^{\prime}=0}^{\infty} \oint_{\gamma_{P, n+1}} d w_{n+1}\left\{\left.\frac{1}{H_{P, n-1}^{W+}-z_{n+1}}(-) H_{I}^{W}\right|_{n} ^{n-1}\right\}^{l} \frac{1}{H_{P, n-1}^{W+}-z_{n+1}} \times \\
\quad \times\left(\Gamma_{P, n-1}+\left.\Delta^{\prime} \Gamma_{P}\right|_{n} ^{n-1}\right)_{i}\left\{\left.\frac{1}{H_{P, n-1}^{W+}-w_{n+1}}(-) H_{I}^{W}\right|_{n} ^{n-1}\right\}^{l^{\prime}} \frac{1}{H_{P, n-1}^{W+}-w_{n+1}} \phi_{P, n-1}
\end{aligned}
$$


where $\mathcal{R}_{P, n-1}$ is the term proportional to $\left.\mathcal{I}\right|_{n} ^{n-1}$. Using Lemma 3.12 we can estimate

$$
\left\|\mathcal{R}_{P, n-1}\right\| \leq \sigma_{n+1} \cdot \frac{1}{1-c_{2}|\lambda|^{\frac{1}{2}}} c_{2}|\lambda|^{\frac{1}{2}} \cdot \frac{1}{1-c_{2}|\lambda|^{\frac{1}{2}}} \cdot \frac{c_{0}}{\sigma_{n+1}} .
$$

(We recall that according to our notation the numerical factor $-\frac{1}{2 \pi i}$ is hidden in the symbol $\oint_{\gamma_{P, n}}$ ). Next we split the term in (C.6) into

$$
\begin{aligned}
\sum_{l=0}^{\infty}\left\{\left.\frac{1}{H_{P, n-1}^{W+}-z_{n+1}}(-) H_{I}^{W}\right|_{n} ^{n-1}\right\}^{l} \frac{1}{H_{P, n-1}^{W+}-z_{n+1}}\left(\Gamma_{P, n-1}+\left.\Delta^{\prime} \Gamma_{P}\right|_{n} ^{n-1}\right)_{i} \phi_{P, n-1} \\
\quad+\sum_{l=0}^{\infty} \sum_{l^{\prime}=1}^{\infty} \oint_{\gamma_{P, n+1}} d w_{n+1}\left\{\left.\frac{1}{H_{P, n-1}^{W+}-z_{n+1}}(-) H_{I}^{W}\right|_{n} ^{n-1}\right\}^{l} \frac{1}{H_{P, n-1}^{W+}-z_{n+1}} \times \\
\quad \times\left(\Gamma_{P, n-1}+\left.\Delta^{\prime} \Gamma_{P}\right|_{n} ^{n-1}\right)_{i}\left\{\left.\frac{1}{H_{P, n-1}^{W+}-w_{n+1}}(-) H_{I}^{W}\right|_{n} ^{n-1}\right\}^{l^{\prime}} \frac{1}{H_{P, n-1}^{W+}-w_{n+1}} \phi_{P, n-1}
\end{aligned}
$$

Term (C.9)

We re-state this term:

$$
\sum_{l=0}^{\infty}\left\{\left.\frac{1}{H_{P, n-1}^{W+}-z_{n+1}}(-) H_{I}^{W}\right|_{n} ^{n-1}\right\}^{l} \frac{1}{H_{P, n-1}^{W+}-z_{n+1}}\left(\Gamma_{P, n-1}+\left.\Delta^{\prime} \Gamma_{P}\right|_{n} ^{n-1}\right)_{i} \phi_{P, n-1}
$$

As for the part of (C.11) proportional to $\left(\left.\Delta^{\prime} \Gamma_{P}\right|_{n} ^{n-1}\right)_{i}:=\left(-\nabla E_{P, n-1}+\nabla E_{P, n}+\left.\mathcal{L}\right|_{n} ^{n-1}\right)_{i}$, notice that:

1. In $\left(\left.\mathcal{L}\right|_{n} ^{n-1}\right)_{i}$ only the term proportional to the creation operator, i.e., $\left(\left.\mathcal{L}\right|_{n} ^{n-1}\right)_{i}^{(+)}$, gives a nonzero contribution. This contribution can be bounded by (see Lemma 3.12)

$$
\sup _{z_{n+1} \in \tilde{\gamma}_{P, n+1}}\left\{\sum_{l=0}^{\infty}\left\|\left\{\left.\frac{1}{H_{P, n-1}^{W+}-z_{n+1}}(-) H_{I}^{W}\right|_{n} ^{n-1}\right\}\right\|^{l}\left\|\frac{1}{H_{P, n-1}^{W+}-z_{n+1}}\left(\left.\mathcal{L}\right|_{n} ^{n-1}\right)_{i}^{(+)} \phi_{P, n-1}\right\|\right\} \leq \frac{1}{1-c_{2}|\lambda|^{\frac{1}{2}}} \cdot c_{2}|\lambda|^{\frac{1}{2}} .(\mathrm{C}
$$

2. The contribution coming from $-\nabla E_{P, n-1}+\nabla E_{P, n}$ can be bounded by

$$
\begin{array}{r}
\sup _{z_{n+1} \in \tilde{\gamma}_{P, n+1}}\left\{\sum_{l=0}^{\infty}\left\|\left\{\left.\frac{1}{H_{P, n-1}^{W+}-z_{n+1}}(-) H_{I}^{W}\right|_{n} ^{n-1}\right\}\right\|^{l}\left|\frac{1}{E_{P, n-1}+\left.\Delta c_{P}\right|_{n} ^{n-1}-z_{n+1}}\right|\right\} c_{1}\left[\lambda^{2} \sigma_{n-1}+\left\|\frac{\hat{\phi}_{P, n}}{\left\|\hat{\phi}_{P, n}\right\|}-\frac{\phi_{P, n-1}}{\left\|\phi_{P, n-1}\right\|}\right\|\right] \\
\leq \frac{1}{1-c_{2}|\lambda|^{\frac{1}{2}}} \cdot \frac{c_{0}}{\sigma_{n+1}} \cdot c_{1} \cdot\left[\lambda^{2} \sigma_{n-1}+4|\lambda|^{\frac{1}{4}} \sigma_{n-1}^{1-\delta}\right] .(\mathrm{C} .
\end{array}
$$

Here we have used (3.19), Lemma 3.12, and a-ii), a-iii). (Notice that $\left\|\hat{\phi}_{P, n}\right\|=\left\|\phi_{P, n}\right\|$ and $\left\|\phi_{P, n-1}\right\| \geq\left\|\phi_{P, n}\right\|$ because of the definitions in (3.18)).

As for the term in (C.11) proportional to $\Gamma_{P, n-1}$, its norm is bounded by

$$
\begin{aligned}
& \sum_{l=0}^{\infty}\left\|\left.\frac{1}{H_{P, n-1}^{W+}-z_{n+1}}(-) H_{I}^{W}\right|_{n} ^{n-1}\right\|^{l}\left\|\frac{1}{H_{P, n-1}^{W+}-z_{n+1}}\left(\Gamma_{P, n-1}\right)_{i} \phi_{P, n-1}\right\| \\
& \leq \sum_{l=0}^{\infty}\left\|\left.\frac{1}{H_{P, n-1}^{W+}-z_{n+1}}(-) H_{I}^{W}\right|_{n} ^{n-1}\right\|^{l} \sup _{z_{n}^{\prime} \in \tilde{\gamma}_{P, n}}\left\|\frac{1}{H_{P, n-1}^{W}-z_{n}^{\prime}}\left(\Gamma_{P, n-1}\right)_{i} \phi_{P, n-1}\right\| \leq \frac{1}{1-c_{2}|\lambda|^{\frac{1}{2}}} \frac{1}{\sigma_{n-1}^{\delta}},
\end{aligned}
$$


where in the first step we applied the maximal modulus principle (Lemma 3.16) after using the gap estimate (3.11) and the fact that $\Gamma_{P, n-1} \phi_{P, n-1}$ is orthogonal to $\phi_{P, n-1}$ (as well as (3.54), (3.55)) to ensure the holomorphy of the expression. It was essential here that $\operatorname{Re} z_{n+1}-\left.\Delta c_{P}\right|_{n} ^{n-1} \leq \operatorname{Re} z_{n}^{\prime}$. In the last step we used the induction hypothesis a-i). (Cf. Remark 4.2 for a similar argument).

Summing up, we have

$$
\|(C .9)\| \leq \frac{1}{1-c_{2}|\lambda|^{\frac{1}{2}}} \cdot c_{2}|\lambda|^{\frac{1}{2}}+\frac{1}{1-c_{2}|\lambda|^{\frac{1}{2}}} \cdot \frac{c_{0}}{\sigma_{n+1}} \cdot c_{1} \cdot\left[\lambda^{2} \sigma_{n-1}+4|\lambda|^{\frac{1}{4}} \sigma_{n-1}^{1-\delta}\right]+\left(\frac{1}{1-c_{2}|\lambda|^{\frac{1}{2}}}\right) \frac{1}{\sigma_{n-1}^{\delta}} .
$$

$\operatorname{Term}(C .10)$

Firstly, we notice that the norm of the term proportional to $\left.\Delta^{\prime} \Gamma_{P}\right|_{n} ^{n-1}$, i.e.,

$$
\begin{aligned}
& \sum_{l=0}^{\infty} \sum_{l^{\prime}=1}^{\infty} \oint_{\gamma_{P, n+1}} d w_{n+1}\left\{\left.\frac{1}{H_{P, n-1}^{W+}-z_{n+1}}(-) H_{I}^{W}\right|_{n} ^{n-1}\right\}^{l} \frac{1}{H_{P, n-1}^{W+}-z_{n+1}}\left(\left.\Delta^{\prime} \Gamma_{P}\right|_{n} ^{n-1}\right)_{i} \times \\
& \times\left.\left\{\left.\frac{1}{H_{P, n-1}^{W+}-w_{n+1}}(-) H_{I}^{W}\right|_{n} ^{n-1}\right\}^{l^{\prime}-1} \frac{1}{H_{P, n-1}^{W+}-w_{n+1}}(-) H_{I}^{W}\right|_{n} ^{n-1} \frac{1}{H_{P, n-1}^{W+}-w_{n+1}} \phi_{P, n-1},
\end{aligned}
$$

is bounded by

$$
\begin{aligned}
& \sigma_{n+1} \frac{1}{1-c_{2}|\lambda|^{\frac{1}{2}}}\left\{\frac{c_{0}}{\sigma_{n+1}} c_{1}\left[\lambda^{2} \sigma_{n-1}+\left\|\frac{\hat{\phi}_{P, n}}{\left\|\hat{\phi}_{P, n}\right\|}-\frac{\phi_{P, n-1}}{\left\|\phi_{P, n-1}\right\|}\right\|\right]+c_{2}|\lambda|^{\frac{1}{2}}\right\}\left(\frac{1}{1-c_{2}|\lambda|^{\frac{1}{2}}}\right) c_{2}|\lambda|^{\frac{1}{2}} \frac{c_{0}}{\sigma_{n+1}} \\
& \leq c_{0} c_{2}|\lambda|^{\frac{1}{2}}\left(\frac{1}{1-c_{2}|\lambda|^{\frac{1}{2}}}\right)^{2}\left\{\frac{c_{0}}{\sigma_{n+1}} c_{1}\left[\lambda^{2} \sigma_{n-1}+4|\lambda|^{\frac{1}{4}} \sigma_{n-1}^{1-\delta}\right]+c_{2}|\lambda|^{\frac{1}{2}}\right\},
\end{aligned}
$$

where we have used (3.19), a-ii), a-iii) and Lemma 3.12.

As for the term proportional to $\Gamma_{P, n-1}$, i.e.,

$$
\begin{array}{r}
\sum_{l=0}^{\infty} \sum_{l^{\prime}=1}^{\infty} \oint_{\gamma_{P, n+1}} d w_{n+1}\left\{\left.\frac{1}{H_{P, n-1}^{W+}-z_{n+1}}(-) H_{I}^{W}\right|_{n} ^{n-1}\right\}^{l} \frac{1}{H_{P, n-1}^{W+}-z_{n+1}}\left(\Gamma_{P, n-1}\right)_{i} \times \\
\times\left.\left\{\left.\frac{1}{H_{P, n-1}^{W+}-w_{n+1}}(-) H_{I}^{W}\right|_{n} ^{n-1}\right\}^{l^{\prime}-1} \frac{1}{H_{P, n-1}^{W+}-w_{n+1}}(-) H_{I}^{W}\right|_{n} ^{n-1} \frac{1}{H_{P, n-1}^{W+}-w_{n+1}} \phi_{P, n-1},
\end{array}
$$

for each summand in the series

$$
\left.\sum_{l^{\prime}=1}^{\infty}\left\{\left.\frac{1}{H_{P, n-1}^{W+}-w_{n+1}}(-) H_{I}^{W}\right|_{n} ^{n-1}\right\}^{l^{\prime}-1} \frac{1}{H_{P, n-1}^{W+}-w_{n+1}}(-) H_{I}^{W}\right|_{n} ^{n-1} \frac{1}{H_{P, n-1}^{W+}-w_{n+1}} \phi_{P, n-1}
$$

consider the first operator $\left.H_{I}^{W}\right|_{n} ^{n-1}$ from the right

$$
\left.H_{I}^{W}\right|_{n} ^{n-1}=\frac{1}{2}\left(\Gamma_{P, n-1} \cdot\left(\left.\mathcal{L}\right|_{n} ^{n-1}+\left.\mathcal{I}\right|_{n} ^{n-1}\right)+\text { h.c. }\right)+\left(\left.H_{I}^{W}\right|_{n} ^{n-1}\right)_{\text {quad }} .
$$

It can be re-written as

$$
\left.H_{I}^{W}\right|_{n} ^{n-1}=\left(\left.\mathcal{L}\right|_{n} ^{n-1}+\left.\mathcal{I}\right|_{n} ^{n-1}\right) \cdot \Gamma_{P, n-1}+\Delta\left(\left.H_{I}^{W}\right|_{n} ^{n-1}\right)_{\operatorname{mix}}+\left(\left.H_{I}^{W}\right|_{n} ^{n-1}\right)_{\text {quad }} .
$$

The contribution proportional to $\Delta\left(\left.H_{I}^{W}\right|_{n} ^{n-1}\right)_{\text {mix }}+\left(\left.H_{I}^{W}\right|_{n} ^{n-1}\right)_{\text {quad }}$ in (C.18), i.e.,

$$
\begin{aligned}
\sum_{l=0}^{\infty} \sum_{l^{\prime}=1}^{\infty} \oint_{\gamma_{P, n+1}} d w_{n+1}\left\{\left.\frac{1}{H_{P, n-1}^{W+}-z_{n+1}}(-) H_{I}^{W}\right|_{n} ^{n-1}\right\}^{l} \frac{1}{H_{P, n-1}^{W+}-z_{n+1}}\left(\Gamma_{P, n-1}\right)_{i} \times \\
\times\left\{\frac{1}{H_{P, n-1}^{W+}-w_{n+1}}(-)\left(\left.H_{I}^{W}\right|_{n} ^{n-1}\right)\right\}^{l^{\prime}-1} \frac{1}{H_{P, n-1}^{W+}-w_{n+1}} \times \\
\times(-)\left\{\Delta\left(\left.H_{I}^{W}\right|_{n} ^{n-1}\right)_{\mathrm{mix}}+\left(\left.H_{I}^{W}\right|_{n} ^{n-1}\right)_{\mathrm{quad}}\right\} \frac{1}{H_{P, n-1}^{W+}-w_{n+1}} \phi_{P, n-1}
\end{aligned}
$$


is bounded in norm by

$$
\sigma_{n+1} \frac{1}{1-c_{2}|\lambda|^{\frac{1}{2}}} \frac{c_{0}}{\sigma_{n+1}} \frac{1}{1-c_{2}|\lambda|^{\frac{1}{2}}}\left(c_{4}|\lambda|^{\frac{1}{2}}+c_{3}|\lambda|\right) \sigma_{n-1} \frac{c_{0}}{\sigma_{n+1}} \leq c_{0}^{2}\left(c_{4}+c_{3}\right)|\lambda|^{\frac{1}{2}} \frac{\sigma_{n-1}}{\sigma_{n+1}}\left(\frac{1}{1-c_{2}|\lambda|^{\frac{1}{2}}}\right)^{2},
$$

where we have used Lemma 3.12.

As for the contribution proportional to $\left(\left.\mathcal{L}\right|_{n} ^{n-1}+\left.\mathcal{I}\right|_{n} ^{n-1}\right) \cdot \Gamma_{P, n-1}$, i.e.,

$$
\begin{array}{r}
\sum_{l=0}^{\infty} \sum_{l^{\prime}=1}^{\infty} \oint_{\gamma_{P, n+1}} d w_{n+1}\left\{\left.\frac{1}{H_{P, n-1}^{W+}-z_{n+1}}(-) H_{I}^{W}\right|_{n} ^{n-1}\right\}^{l} \frac{1}{H_{P, n-1}^{W+}-z_{n+1}}\left(\Gamma_{P, n-1}\right)_{i} \times \\
\times\left\{\left.\frac{1}{H_{P, n-1}^{W+}-w_{n+1}}(-) H_{I}^{W}\right|_{n} ^{n-1}\right\}^{l^{\prime}-1} \frac{1}{H_{P, n-1}^{W+}-w_{n+1}}(-)\left(\left.\mathcal{L}\right|_{n} ^{n-1}+\left.\mathcal{I}\right|_{n} ^{n-1}\right) \cdot \Gamma_{P, n-1} \times \\
\times \frac{1}{H_{P, n-1}^{W+}-w_{n+1}} \phi_{P, n-1},
\end{array}
$$

we split it into two pieces. The norm of the summand proportional to $\left.\mathcal{I}\right|_{n} ^{n-1} \cdot \Gamma_{P, n-1}$ can be easily estimated in terms of

$$
\begin{aligned}
\sigma_{n+1} \frac{1}{1-c_{2}|\lambda|^{\frac{1}{2}}} \cdot \frac{1}{1-c_{2}|\lambda|^{\frac{1}{2}}} \cdot \frac{c_{0}}{\sigma_{n+1}} c_{I}|\lambda|^{2} \sigma_{n-1} & \sum_{j=1}^{3}\left\|\frac{1}{H_{P, n-1}^{W+}-w_{n+1}}\left(\Gamma_{P, n-1}\right)_{j} \phi_{P, n-1}\right\| \frac{c_{0}}{\sigma_{n+1}} \\
& \leq c_{0}^{2}\left(\frac{1}{1-c_{2}|\lambda|^{\frac{1}{2}}}\right)^{2} \cdot\left(\frac{\sigma_{n-1}}{\sigma_{n+1}}\right) \cdot c_{I}|\lambda|^{2} \cdot \frac{3}{\sigma_{n-1}^{\delta}}
\end{aligned}
$$

where we have used that $\left.|\mathcal{I}|_{n}^{n-1}\left|\leq c_{I}\right| \lambda\right|^{2} \sigma_{n-1}, c_{I}$ being a universal constant, Lemma 3.12, the maximal modulus principle (Lemma 3.16 and Remark 4.2) and the induction hypothesis a-i). As for the other summand, making use of the direct integral representations from Subsection 3.4 we can write

$$
\begin{aligned}
& \frac{1}{H_{P, n-1}^{W+}-w_{n+1}}\left(\left.\mathcal{L}\right|_{n} ^{n-1} \cdot \Gamma_{P, n-1}\right) \frac{1}{H_{P, n-1}^{W+}-w_{n+1}} \phi_{P, n-1} \\
& \quad \simeq O\left(\sigma_{n+1}^{-1}\right)(-\lambda) \int_{\left.\mathcal{A}\right|_{n} ^{n-1}}^{\oplus} \frac{d^{3} k}{\sqrt{2}|k|^{3 / 2}} \frac{k_{i}}{\alpha_{P, n-1}(\hat{k})} \frac{1}{\left[H_{P, n-1}^{W+}\right]_{k}-w_{n+1}}\left(\Gamma_{P, n-1}\right)_{i} \phi_{P, n-1},
\end{aligned}
$$

where $O\left(\sigma_{n-1}^{-1}\right):=\left(E_{P, n-1}+\left.\Delta c_{P}\right|_{n} ^{n-1}-w_{n+1}\right)^{-1}$. Now by (3.72) and the induction hypothesis combined with Remark 4.2 we get

$$
\|(C .26)\| \leq c|\lambda|\left(\frac{\sigma_{n-1}}{\sigma_{n+1}}\right)|\ln \epsilon|^{1 / 2} \frac{1}{\sigma_{n-1}^{\delta}} .
$$

Hence the norm of the summand in (C.24) proportional to $\left(\left.\mathcal{L}\right|_{n} ^{n-1} \cdot \Gamma_{P, n-1}\right)$ is bounded by

$$
\sigma_{n+1} \frac{1}{1-c_{2}|\lambda|^{\frac{1}{2}}} \cdot \frac{1}{1-c_{2}|\lambda|^{\frac{1}{2}}} \cdot \frac{c_{0}}{\sigma_{n+1}} c|\lambda|\left(\frac{\sigma_{n-1}}{\sigma_{n+1}}\right)|\ln \epsilon|^{1 / 2} \frac{1}{\sigma_{n-1}^{\delta}}=c_{0}\left(\frac{1}{1-c_{2}|\lambda|^{\frac{1}{2}}}\right)^{2} c|\lambda|\left(\frac{\sigma_{n-1}}{\sigma_{n+1}}\right)|\ln \epsilon|^{1 / 2} \frac{1}{\sigma_{n-1}^{\delta}} \text {. (C. }
$$


The sum of all contributions (C.8), (C.15), (C.17), (C.23), (C.25), and (C.28), yields

$$
\begin{aligned}
& \sigma_{n+1} \cdot \frac{1}{1-c_{2}|\lambda|^{\frac{1}{2}}} c_{2}|\lambda|^{\frac{1}{2}} \cdot \frac{1}{1-c_{2}|\lambda|^{\frac{1}{2}}} \cdot \frac{c_{0}}{\sigma_{n+1}} \\
& +\frac{1}{1-c_{2}|\lambda|^{\frac{1}{2}}} \cdot|\lambda|^{\frac{1}{2}} c_{2}+\frac{1}{1-c_{2}|\lambda|^{\frac{1}{2}}} \cdot \frac{c_{0}}{\sigma_{n+1}} \cdot c_{1} \cdot\left[\lambda^{2} \sigma_{n-1}+4|\lambda|^{\frac{1}{4}} \sigma_{n-1}^{1-\delta}\right]+\left(\frac{1}{1-c_{2}|\lambda|^{\frac{1}{2}}}\right) \frac{1}{\sigma_{n-1}^{\delta}} \\
& +c_{0} c_{2}|\lambda|^{\frac{1}{2}}\left(\frac{1}{1-c_{2}|\lambda|^{\frac{1}{2}}}\right)^{2}\left\{\frac{c_{0}}{\sigma_{n+1}} c_{1}\left[\lambda^{2} \sigma_{n-1}+4|\lambda|^{\frac{1}{4}} \sigma_{n-1}^{1-\delta}\right]+c_{2}|\lambda|^{\frac{1}{2}}\right\} \\
& +c_{0}^{2}\left(c_{4}+c_{3}\right)|\lambda|^{\frac{1}{2}} \frac{\sigma_{n-1}}{\sigma_{n+1}}\left(\frac{1}{1-c_{2}|\lambda|^{\frac{1}{2}}}\right)^{2}+c_{0}^{2}\left(\frac{1}{1-c_{2}|\lambda|^{\frac{1}{2}}}\right)^{2} \cdot\left(\frac{\sigma_{n-1}}{\sigma_{n+1}}\right) \cdot c_{I}|\lambda|^{2} \cdot \frac{3}{\sigma_{n-1}^{\delta}} \\
& +c_{0}\left(\frac{1}{1-c_{2}|\lambda|^{\frac{1}{2}}}\right)^{2} c|\lambda|\left(\frac{\sigma_{n-1}}{\sigma_{n+1}}\right)|\ln \epsilon|^{1 / 2} \frac{1}{\sigma_{n-1}^{\delta}} \leq \frac{C^{(i)}}{\sigma_{n-1}^{\delta}}
\end{aligned}
$$

for some universal constant $C^{(i)}$, where we made use of $|\lambda| \leq \epsilon^{8}$. (The power eight is needed in the second line above in the term involving $\left.|\lambda|^{1 / 4}\right)$. Thus, by setting $\epsilon(\delta):=\min \left\{\frac{1}{2} ;\left(1 / \mathcal{C}^{(i)}\right)^{\frac{1}{\delta}}\right\}$ and choosing $\lambda_{0}^{(i)} \equiv \lambda_{0}^{(i)}(\epsilon(\delta))>0$ (within all the earlier restrictions) such that

$$
\lambda_{0}^{(i)} \leq \epsilon(\delta)^{8}
$$

we have proven that c-i) is implied by a-i), a-ii), a-iii) for all $|\lambda| \in\left(0, \lambda_{0}^{(i)}\right]$ and $\epsilon \in(0, \epsilon(\delta)]$ s.t. $|\lambda| \leq \epsilon^{8}$.

Claim-II) (Here we show the implication: $c$-i) $\rightarrow c$-ii))

We choose $\epsilon$ and $\lambda$ as specified above. We set $m:=n+1$ and start from the difference

$$
\begin{aligned}
\hat{\phi}_{P, n+1}-\phi_{P, n}= & \sum_{j=1}^{\infty} \oint_{\gamma_{P, m+1}} d w_{m+1}\left\{\left.\frac{1}{H_{P, m-1}^{W+}-w_{m+1}}(-) H_{I}^{W}\right|_{m} ^{m-1}\right\}^{j-1} \times \\
& \times \frac{1}{H_{P, m-1}^{W+}-w_{m+1}}(-)\left[\left(\left.\mathcal{L}\right|_{m} ^{m-1}+\left.\mathcal{I}\right|_{m} ^{m-1}\right) \cdot \Gamma_{P, m-1}+\Delta\left(\left.H_{I}^{W+}\right|_{m} ^{m-1}\right)_{\operatorname{mix}}+\left(\left.H_{I}^{W}\right|_{m} ^{m-1}\right)_{\mathrm{quad}}\right] \times \\
& \times \frac{1}{H_{P, m-1}^{W+}-w_{m+1}} \phi_{P, m-1} .
\end{aligned}
$$

By Lemma 3.12, the contribution proportional to $\Delta\left(\left.H_{I}^{W}\right|_{m} ^{m-1}\right)_{\text {mix }}+\left(\left.H_{I}^{W}\right|_{m} ^{m-1}\right)_{\text {quad }}$ can be bounded in norm by

$$
\sigma_{m+1} \frac{1}{1-c_{2}|\lambda|^{\frac{1}{2}}} \cdot\left(c_{4}|\lambda|^{\frac{1}{2}}+c_{3}|\lambda|\right) \sigma_{m-1} \frac{c_{0}}{\sigma_{m+1}} .
$$

(Clearly, the operator norms involved in these estimates are on $B\left(\mathcal{F}_{m}\right)$ rather than on $B\left(\mathcal{F}_{n}\right)$ ). We can rewrite the rest as follows

$$
\begin{aligned}
&-\sum_{i^{\prime}=1}^{3}\left(\left.\mathcal{I}\right|_{m} ^{m-1}\right)_{i^{\prime}} \sum_{j=1}^{\infty} \oint_{\gamma_{P, m+1}} d w_{m+1}\left(\frac{1}{E_{P, m-1}^{+}-w_{m+1}}\right)\left\{\left.\frac{1}{H_{P, m-1}^{W+}-w_{m+1}}(-) H_{I}^{W}\right|_{m} ^{m-1}\right\}^{j-1} \times \\
& \times \frac{1}{H_{P, m-1}^{W+}-w_{m+1}}\left(\Gamma_{P, m-1}\right)_{i^{\prime}} \phi_{P, m-1} \\
&-\sum_{i^{\prime}=1}^{3} \sum_{j=1}^{\infty} \oint_{\gamma_{P, m+1}} d w_{m+1}\left(\frac{1}{E_{P, m-1}^{+}-w_{m+1}}\right)\left\{\left.\frac{1}{H_{P, m-1}^{W+}-w_{m+1}}(-) H_{I}^{W}\right|_{m} ^{m-1}\right\}^{j-1} \times \\
& \times \frac{1}{H_{P, m-1}^{W+}-w_{m+1}}\left(\left.\mathcal{L}\right|_{m} ^{m-1}\right)_{i^{\prime}}^{(+)}\left(\Gamma_{P, m-1}\right)_{i^{\prime}} \phi_{P, m-1},
\end{aligned}
$$


where $E_{P, m-1}^{+}:=E_{P, m-1}+\left.\Delta c_{P}\right|_{m} ^{m-1}$. Using that $\left|\left(\left.\mathcal{I}\right|_{m} ^{m-1}\right)_{i^{\prime}}\right| \leq c_{I}|\lambda|^{2} \sigma_{m-1}$ and applying the maximal modulus principle (Lemma 3.16 and Remark 4.2) and c-i) to (C.33), we obtain

$$
\|(C .33)\| \leq \frac{3 c_{0} \cdot c_{I}|\lambda|^{2} \sigma_{m-1}}{1-c_{2}|\lambda|^{\frac{1}{2}}} \frac{1}{\sigma_{m-1}^{\delta}} .
$$

Next, arguing as in (C.26), (C.27) above and making use of c-i) we obtain

$$
\left\|\frac{1}{H_{P, m-1}^{W+}-w_{m+1}}\left(\left.\mathcal{L}\right|_{m} ^{m-1}\right)_{i^{\prime}}^{(+)}\left(\Gamma_{P, m-1}\right)_{i^{\prime}} \phi_{P, m-1}\right\| \leq c|\lambda| \sigma_{m-1}|\ln \epsilon|^{1 / 2} \frac{1}{\sigma_{m-1}^{\delta}},
$$

and therefore

$$
\|(C .34)\| \leq \frac{3 c_{0}}{1-c_{2}|\lambda|^{\frac{1}{2}}} c|\lambda| \sigma_{m-1}|\ln \epsilon|^{1 / 2} \frac{1}{\sigma_{m-1}^{\delta}} .
$$

Summing up, we have

$$
\begin{aligned}
\left\|\hat{\phi}_{P, n+1}-\phi_{P, n}\right\| \leq & \frac{c_{0}}{1-c_{2}|\lambda|^{\frac{1}{2}}} \cdot\left(c_{4}|\lambda|^{\frac{1}{2}}+c_{3}|\lambda|\right) \sigma_{m-1} \\
& \quad+\frac{3 c_{0} \cdot c_{I}|\lambda|^{2} \sigma_{m-1}}{1-c_{2}|\lambda|^{\frac{1}{2}}} \frac{1}{\sigma_{m-1}^{\delta}}+\frac{3 c_{0}}{1-c_{2}|\lambda|^{\frac{1}{2}}} c|\lambda| \sigma_{m-1}|\ln \epsilon|^{1 / 2} \frac{1}{\sigma_{m-1}^{\delta}} \\
\leq & C^{(i i)|\lambda|^{\frac{1}{2}} \sigma_{m-1}^{1-\delta}=C^{(i i)}|\lambda|^{\frac{1}{2}} \sigma_{n}^{1-\delta}}
\end{aligned}
$$

for some universal constant $C^{(i i)}$, and finally

$$
\left\|\hat{\phi}_{P, n+1}-\phi_{P, n}\right\| \leq|\lambda|^{\frac{1}{4}} \sigma_{n}^{1-\delta}
$$

for $|\lambda| \in\left(0, \lambda_{0}^{(i i)}\right]$, where $\lambda_{0}^{(i i)} \equiv \min \left\{\lambda_{0}^{(i)},\left[\frac{1}{C^{(i i)}}\right]^{4}\right\}$.

ClaIm-III) (Here we show the implication: $c$-ii) and a-iii) $\rightarrow c$-iii))

We choose $\epsilon$ and $\lambda$ within the restrictions specified below formulas (C.30) and (C.39). By relation (3.18)

$$
\left\|\phi_{P, n+1}-\hat{\phi}_{P, n+1}\right\|=\left\|\left(W_{P, n+1} \widetilde{W}_{P, n+1}^{*}-1\right) \hat{\phi}_{P, n+1}\right\| .
$$

Using Lemma D.5 and relation (3.19), the r.h.s of (C.40) can be estimated less than

$$
\begin{aligned}
c|\lambda| \mid \nabla E_{P, n+1} & -\nabla E_{P, n}\left\|\ln \sigma_{n+1} \mid\right\| \hat{\phi}_{P, n+1} \| \\
& \leq c|\lambda| c_{1}\left|\ln \sigma_{n+1}\right|\left[\lambda^{2} \sigma_{n}+\left\|\frac{\hat{\phi}_{P, n+1}}{\left\|\hat{\phi}_{P, n+1}\right\|}-\frac{\phi_{P, n}}{\left\|\phi_{P, n}\right\|}\right\|\right]\left\|\hat{\phi}_{P, n+1}\right\| \\
& \leq c|\lambda| c_{1}\left|\ln \sigma_{n+1}\right|\left[\lambda^{2} \sigma_{n}\left\|\hat{\phi}_{P, n+1}\right\|+\left\|\hat{\phi}_{P, n+1}-\phi_{P, n}\right\|+\left|\left\|\phi_{P, n}\right\|-\left\|\hat{\phi}_{P, n+1}\right\|\right|\right] \\
& \leq c|\lambda| c_{1}\left|\ln \sigma_{n+1}\right|\left[\lambda^{2} \sigma_{n}+2|\lambda|^{\frac{1}{4}} \sigma_{n}^{1-\delta}\right] \leq C^{\prime(i i i)}|\lambda|^{\frac{5}{4}} \sigma_{n}^{1-\delta}\left|\ln \sigma_{n+1}\right|,
\end{aligned}
$$

where we made use of c-ii), the fact that $\left\|\phi_{P, n+1}\right\| \leq 1$, and $C^{\prime(i i i)}$ is some universal constant. Furthermore, we can write

$$
\begin{aligned}
C^{\prime(i i i)}|\lambda|^{\frac{5}{4}} \sigma_{n}^{1-\delta}\left|\ln \sigma_{n+1}\right| & \leq C^{\prime(i i i)}|\lambda|^{\frac{5}{4}} \frac{\sigma_{n+1}^{1-\delta}}{\epsilon^{1-\delta}}\left|\ln \sigma_{n+1}\right|=C^{\prime(i i i)}\left(|\lambda|^{1 / 4} \sigma_{n+1}^{1 / 4}\right)|\lambda| \frac{\sigma_{n+1}^{3 / 4-\delta}}{\epsilon^{1-\delta}}\left|\ln \sigma_{n+1}\right| \\
& \leq C^{\prime(i i i)}|\lambda|^{1 / 2}\left(|\lambda|^{1 / 4} \sigma_{n+1}^{1 / 2}\right) \frac{|\lambda|^{1 / 2}}{\epsilon} \sigma_{n+1}^{1 / 4}\left|\ln \sigma_{n+1}\right| \leq C^{(i i i)}|\lambda|^{1 / 2}\left(|\lambda|^{1 / 4} \sigma_{n+1}^{1 / 2}\right),
\end{aligned}
$$


for some universal constant $C^{(i i i)}\left(=c C^{\prime(i i i)}\right)$, where we used that $\frac{|\lambda|^{\frac{1}{4}}}{\epsilon} \leq 1$ and $\sigma_{n+1}^{1 / 4}\left|\ln \sigma_{n+1}\right| \leq c$. Altogether, we have by (C.40), (C.41), (C.42)

$$
\left\|\phi_{P, n+1}-\hat{\phi}_{P, n+1}\right\| \leq C^{(i i i)}|\lambda|^{1 / 2}\left(|\lambda|^{1 / 4} \sigma_{n+1}^{1 / 2}\right) .
$$

Thus we obtain by the inverse triangle inequality, (C.43) and c-ii)

$$
\left\|\phi_{P, n+1}\right\| \geq\left\|\phi_{P, n}\right\|-\left\|\phi_{P, n+1}-\hat{\phi}_{P, n+1}\right\|-\left\|\hat{\phi}_{P, n+1}-\phi_{P, n}\right\| \geq\left\|\phi_{P, n}\right\|-\left(C^{(i i i)}|\lambda|^{1 / 2}+1\right)|\lambda|^{\frac{1}{4}} \sigma_{n}^{1 / 2} .
$$

We choose $\lambda_{0}$ s.t. $C^{(i i i)}\left|\lambda_{0}\right|^{1 / 2} \leq 1$ and obtain from (C.44) and a-iii)

$$
\left\|\phi_{P, n+1}\right\| \geq 1-2 \sum_{j=0}^{n}\left\{|\lambda|^{\frac{1}{4}} \sigma_{j}^{1 / 2}\right\} \geq 1-2 \frac{|\lambda|^{1 / 4}}{1-\epsilon^{1 / 2}} .
$$

We obtain from the last formula that c-ii) and a-iii) imply c-iii) provided that

$$
|\lambda| \in\left(0, \lambda_{0}\right], \quad \lambda_{0} \equiv \min \left(\lambda_{0}^{(i i)}, \frac{1}{\left(C^{(i i i)}\right)^{2}},\left(\frac{1-\epsilon(\delta)^{1 / 2}}{4}\right)^{4}\right) .
$$

This concludes the proof.

\section{A pull through identity and its consequences}

We refer to (D.5) below as a pull through identity. It has several useful consequences (see e.g. (C.41) above) which will be studied in this appendix. The analysis of pull-through estimates dates back to [17], but our treatment of technical aspects also profited from [27]. We will use the domain

$$
C^{\infty}\left(H_{P, \text { free }}\right):=\bigcap_{\ell \geq 0} D\left(H_{P, \text { free }}^{\ell}\right)
$$

We also recall the standard bounds, valid for any $P \in S$ and $\ell \in \mathbb{N}$

$$
\left\|H_{P, \text { free }}^{\ell}\left(H_{P, n}+i\right)^{-\ell}\right\| \leq c,
$$

For $\ell=1$ these estimates follow from Lemmas A.1, A.3. For $\ell \geq 1$ we refer to [21, 28, 13] for the standard arguments.

By Lemma A.1, for $\psi \in C^{\infty}\left(H_{P, \text { free }}\right)$ we can define $\mathbb{R}^{3 m} \ni\left(k_{1}, \ldots, k_{m}\right) \mapsto b\left(k_{1}\right) \ldots b\left(k_{m}\right) \psi$ as a vectorvalued distribution on $S\left(\mathbb{R}^{3 m}\right)$ which gives rise to a continuous map on $L_{\omega}^{2}\left(\mathbb{R}^{3}\right)^{\times m}$, where $L_{\omega}^{2}\left(\mathbb{R}^{3}\right):=\{f \in$ $\left.L^{2}\left(\mathbb{R}^{3}\right)||\left\|\left.k\right|^{-1 / 2} f\right\|_{2}<\infty\right\}$. For some vectors $\psi \in C^{\infty}\left(H_{P, \text { free }}\right)$ we can also define $b\left(k_{1}\right) \ldots b\left(k_{m}\right) \psi$ pointwise in $\left(k_{1}, \ldots, k_{m}\right)$ as follows:

Definition D.1. Let $\eta \in C_{0}^{\infty}\left(\mathbb{R}^{3}\right)$ be a spherically symmetric function s.t. $\eta \geq 0, \int \eta\left(k^{\prime}\right) d^{3} k^{\prime}=1$, so that $\eta_{k}^{\varepsilon}\left(k^{\prime}\right):=$ $\varepsilon^{-3} \eta\left(\left(k^{\prime}-k\right) \varepsilon^{-1}\right)$ is an approximating sequence of $k^{\prime} \mapsto \delta\left(k^{\prime}-k\right)$ as $\varepsilon \rightarrow 0$. Then, for any $\left(k_{1}, \ldots, k_{m}\right) \in \mathbb{R}^{3 m}$, we say that $\psi \in C^{\infty}\left(H_{P, \text { free }}\right)$ is in $D\left(b\left(k_{1}\right) \ldots b\left(k_{m}\right)\right)$ if the following limit exists in norm

$$
\left[b\left(k_{1}\right) \ldots b\left(k_{m}\right) \psi\right]:=\lim _{\varepsilon \rightarrow 0} b\left(\eta_{k_{1}}^{\varepsilon}\right) \ldots b\left(\eta_{k_{m}}^{\varepsilon}\right) \psi .
$$

For $m=0$ we set by convention $D\left(b\left(k_{1}\right) \ldots b\left(k_{m}\right)\right):=C^{\infty}\left(H_{P, \text { free }}\right)$.

Lemma D.3 below shows that this definition is consistent with the definition as a distribution and, consequently, that the dependence on $\eta$ is inessential after smearing. In the following lemma we will use the notation $\underline{k}_{m}:=k_{1}+\cdots+k_{m}$ and $|\underline{k}|_{m}:=\left|k_{1}\right|+\cdots+\left|k_{m}\right|$. 
Lemma D.2. [17] Let $|\lambda| \in\left(0, \lambda_{0}\right],|P| \leq P_{\max }=1 / 3$ and $m \in \mathbb{N}_{0}$. Then:

(a) For $k_{1}, \ldots, k_{m} \in \mathbb{R}^{3} \backslash\{0\}$, $m \geq 1$, the following bound holds true

$$
\left\|\frac{1}{E_{P, n}-|\underline{k}|_{m}-H_{P-\underline{k}_{m}, n}}\right\| \leq \frac{c}{|\underline{k}|_{m}}
$$

(b) $\psi_{P, n} \in D\left(b\left(k_{1}\right) \ldots b\left(k_{m}\right)\right)$ for $k_{1}, \ldots, k_{m} \in \mathbb{R}^{3} \backslash\{0\}$ and satisfies

$$
\left[b\left(k_{1}\right) \ldots b\left(k_{m}\right) \psi_{P, n}\right]=\frac{1}{E_{P, n}-|\underline{k}|_{m}-H_{P-\underline{k}_{m}, n}} \sum_{i=1}^{m} v_{\bar{\alpha}}^{\sigma_{n}}\left(k_{i}\right)\left[b\left(k_{1}\right) \ldots \check{i} \ldots b\left(k_{m}\right) \psi_{P, n}\right] .
$$

If $\left|k_{i}\right|=\sigma_{n}$ for some $i=1, \ldots, m$, the corresponding $v_{\bar{\alpha}}^{\sigma_{n}}\left(k_{i}\right)$ must be multiplied by $\frac{1}{2}$.

(c) For any $f_{1}, \ldots f_{m} \in L^{2}\left(\mathbb{R}^{3}\right) \cap L^{1}\left(\mathbb{R}^{3}\right)$, supported away from zero ${ }^{2}$,

$$
\int d^{3} k_{1} \ldots d^{3} k_{m} \bar{f}_{1}\left(k_{1}\right) \ldots \bar{f}_{m}\left(k_{m}\right)\left[b\left(k_{1}\right) \ldots b\left(k_{m}\right) \psi_{P, n}\right]=b\left(f_{1}\right) \ldots b\left(f_{m}\right) \psi_{P, n},
$$

where the l.h.s. is a weak integral and on the r.h.s. the usual definition of the annihilation operators is understood.

Proof. To prove (a) we note that, by definition of $E_{P-\underline{k}_{m}, n}$, we have $H_{P-\underline{k}_{n}, n}-E_{P-\underline{k}_{m}, n} \geq 0$ and by (3.7) $E_{P-\underline{k}_{m}, n}-E_{P, n}+|\underline{k}|_{m} \geq(2 / 3-c|\lambda|)|\underline{k}|_{m}>0$ for $|\underline{k}|_{m} \neq 0$ (cf. also Remark A.11). Therefore $H_{P-\underline{k}_{n}, n}+|\underline{k}|_{m}-E_{P, n} \geq$ $c^{\prime}|\underline{k}|_{m}>0$ and (D.4) follows.

To prove (b) and (c) we first assume that $\left|k_{i}\right| \neq \sigma_{n}, i=1, \ldots, m$, and only in the last part of the proof we will explain how to drop this assumption. We proceed by induction, namely we suppose that for $m^{\prime}=0,1, \ldots, m-1$ the following inductive assumptions hold

a-(b) $\psi_{P, n} \in D\left(b\left(k_{1}\right) \ldots b\left(k_{m^{\prime}}\right)\right)$ for all $k_{1}, \ldots k_{m^{\prime}} \in \mathbb{R}^{3} \backslash\{0\}$ and

$$
\left[b\left(k_{1}\right) \ldots b\left(k_{m^{\prime}}\right) \psi_{P, n}\right]=\frac{1}{E_{P, n}-|\underline{k}|_{m^{\prime}}-H_{P-\underline{k}_{m^{\prime}}, n}} \sum_{i=1}^{m^{\prime}} v_{\bar{\alpha}}^{\sigma}\left(k_{i}\right)\left[b\left(k_{1}\right) \ldots \check{i} \ldots b\left(k_{m^{\prime}}\right) \psi_{P, n}\right] .
$$

a-(c) $\int d^{3} k_{1} \ldots d^{3} k_{m^{\prime}} \bar{f}_{1}\left(k_{1}\right) \ldots \bar{f}_{m^{\prime}}\left(k_{m^{\prime}}\right)\left[b\left(k_{1}\right) \ldots b\left(k_{m^{\prime}}\right) \psi_{P, n}\right]=b\left(f_{1}\right) \ldots b\left(f_{m^{\prime}}\right) \psi_{P, n}$ for any $f_{1}, \ldots f_{m^{\prime}} \in L^{2}\left(\mathbb{R}^{3}\right) \cap$ $L^{1}\left(\mathbb{R}^{3}\right)$ supported away from zero.

For $m=1$ the only content of our inductive assumption is that $\psi_{P, n} \in C^{\infty}\left(H_{P, \text { free }}\right)$, which holds by (D.2). We will conclude from these assumptions statements (b) and (c) of the lemma for $m$, thereby closing the inductive argument. As a preparation, we note that by iteration of (D.7) we get (cf. [17])

$$
\left[b\left(q_{1}\right) \ldots b\left(q_{m-1}\right) \psi_{P, n}\right]=\sum_{\pi \in S_{m-1}}\left\{(-1)^{m-1} \prod_{i=m-1}^{1} \frac{1}{H_{P-\underline{q}_{\pi, i}, n}-E_{P, n}+|\underline{q}|_{\pi, i}} v_{\bar{\alpha}}^{\sigma_{n}}\left(q_{\pi(i)}\right) \psi_{P, n}\right\},
$$

where $\pi \in S_{m-1}$ is any permutation of $(1, \ldots, m-1)$ and $\underline{q}_{\pi, i}=\sum_{i^{\prime}=1}^{i} q_{\pi_{i^{\prime}}},|\underline{q}|_{\pi, i}=\sum_{i^{\prime}=1}^{i}\left|q_{\pi_{i^{\prime}}}\right|$. (We renamed here the variables from $k_{i}$ to $q_{i}$ to facilitate applications in the later part of the proof, where $q_{i}$ will denote smearing variables). By the bound from part (a) of the lemma and a combinatorial argument from [17] we obtain a standard bound

$$
\left\|\left[b\left(q_{1}\right) \ldots b\left(q_{m-1}\right) \psi_{P, n}\right]\right\| \leq \prod_{i=1}^{m-1} \frac{c v_{\bar{\alpha}}^{\sigma_{n}}\left(q_{i}\right)}{\left|q_{i}\right|}
$$

\footnotetext{
${ }^{2}$ For consistency with the discussion above Definition D.1, we note that if $f \in L^{2}\left(\mathbb{R}^{3}\right)$ is supported away from zero, then it is automatically in $L_{\omega}^{2}\left(\mathbb{R}^{3}\right)$.
} 
Making use of a-(c) and (D.9) we also obtain for $k_{1}, \ldots, k_{m-1}$ in any compact set $K \subset \mathbb{R}^{3} \backslash\{0\}$ and any continuous functions $g_{1}, \ldots, g_{m-1}$

$$
\begin{aligned}
\left\|b\left(g_{1} \eta_{k_{1}}^{\varepsilon}\right) \ldots b\left(g_{m-1} \eta_{k_{m-1}}^{\varepsilon}\right) \psi_{P, n}\right\| & \leq \prod_{i=1}^{m-1} \int d^{3} q_{i} \frac{c v_{\bar{\alpha}}^{\sigma_{n}}\left(q_{i}\right)}{\left|q_{i}\right|}\left|g_{i}\left(q_{i}\right)\right| \eta_{k_{i}}^{\varepsilon}\left(q_{i}\right) \\
& \leq \prod_{i=1}^{m-1} \int d^{3} q_{i} \eta\left(q_{i}\right)\left|g_{i}\left(k_{i}+\varepsilon q_{i}\right)\right| \frac{\chi_{\left[\sigma_{n}, \kappa\right)}\left(k_{i}+\varepsilon q_{i}\right)\left|k_{i}+\varepsilon q_{i}\right|^{\bar{\alpha}}}{\left|k_{i}+\varepsilon q_{i}\right|^{3 / 2}} \\
& \leq C_{K, m-1} \prod_{i=1}^{m-1} \sup _{q \in \operatorname{supp} \eta}\left|g_{i}\left(k_{i}+\varepsilon q\right)\right| \leq C_{K, m-1}^{\prime} .
\end{aligned}
$$

The last bound holds uniformly in $k_{1}, \ldots, k_{m-1} \in K$ and in $\varepsilon$ s.t. $\left|k_{i}+\varepsilon q\right|>\frac{1}{2}\left|k_{i}\right|$ for all $q \in \operatorname{supp} \eta$. (The constants $C_{K, m-1}, C_{K, m-1}^{\prime}$ do not depend on $n, \bar{\alpha}$. But the latter constant depends on $g_{i}$ ). The estimate (D.10) will be important for our proof of part (c) of the lemma for $m$ via Lemma D.3.

Let us now proceed to the proof of (D.5). In the following, the expressions $b\left(q_{1}\right) \ldots b\left(q_{m}\right) \psi$, for $\psi \in$ $C^{\infty}\left(H_{P, \text { free }}\right)$, are understood in the sense of distributions unless stated otherwise. More precisely, the following smearing is understood

$$
b\left(f_{m}\right) \ldots b\left(f_{1}\right) \psi=\int d^{3} q_{1} \ldots d^{3} q_{m} \bar{f}_{m}\left(q_{m}\right) \ldots \bar{f}_{1}\left(q_{1}\right) b\left(q_{m}\right) \ldots b\left(q_{1}\right) \psi
$$

with functions $f_{1}, \ldots, f_{m} \in L^{2}\left(\mathbb{R}^{3}\right) \cap L^{1}\left(\mathbb{R}^{3}\right)$ supported away from zero.

By canonical commutation relations, for any $\psi \in C^{\infty}\left(H_{P, \text { free }}\right)$

$$
b(\tilde{k}) H_{P-k, \sigma} \psi=\left(H_{P-k-\tilde{k}, \sigma}+|\tilde{k}|\right) b(\tilde{k}) \psi+v_{\bar{\alpha}}^{\sigma}(\tilde{k}) \psi
$$

holds in the sense of distributions. Next, by iteration of (D.12), we obtain that

$$
b\left(q_{m}\right) \ldots b\left(q_{1}\right) H_{P, n} \psi=\left(H_{P-\underline{q}_{m}, n}+|\underline{q}|_{m}\right) b\left(q_{m}\right) \ldots b\left(q_{1}\right) \psi+\sum_{i=1}^{m} v_{\bar{\alpha}}^{\sigma}\left(q_{i}\right) b\left(q_{m}\right) \ldots \check{i} \ldots b\left(q_{1}\right) \psi,
$$

where analogous smearing as in (D.11) is understood. To move on, we set $\psi \equiv \psi_{P, n}$ in (D.13) and introduce the following notation:

$$
\begin{aligned}
\underline{q} & :=\left(q_{m-1}, \ldots, q_{1}\right), \\
\underline{q}^{i} & :=\left(q_{m-1}, \ldots \check{i} \ldots q_{1}\right), \\
\underline{q}^{i, i^{\prime}} & :=\left(q_{m-1}, \ldots \check{i} \ldots \check{i^{\prime}} \ldots q_{1}\right), \text { for } i \neq i^{\prime}, \\
A_{q}\left(\bar{q}_{m}\right) & :=\left(E_{P, n}-|\underline{q}|_{m-1}-\left|q_{m}\right|-H_{P-q_{m-1}}-q_{m}, n\right), \\
B_{m-1}(\underline{q}) & :=b\left(q_{m-1}\right) \ldots b\left(q_{1}\right), \quad B_{m-1}=1 \text { for } m=1, \\
B_{m-1}^{i}\left(\underline{q}^{i}\right) & :=b\left(q_{m-1}\right) \ldots \check{i} \ldots b\left(q_{1}\right), B_{m-1}^{i}=0 \text { for } m=1, \\
B_{m-1}^{i}\left(\underline{q}^{i, i^{\prime}}\right) & :=b\left(q_{m-1}\right) \ldots \check{i} \ldots \check{i^{\prime}} \ldots b\left(q_{1}\right), B_{m-1}^{i, i^{\prime}}=0 \quad \text { for } m=1,2 .
\end{aligned}
$$

We use $H_{P, n} \psi_{P, n}=E_{P, n} \psi_{P, n}$, and combine the term on the 1.h.s. of (D.13) with the first term on the r.h.s. so as to obtain

$$
A_{\underline{q}}\left(q_{m}\right) b\left(q_{m}\right) B_{m-1}(\underline{q}) \psi_{P, n}=v_{\bar{\alpha}}^{\sigma}\left(q_{m}\right) B_{m-1}(\underline{q}) \psi_{P, n}+\sum_{i=1}^{m-1} v_{\bar{\alpha}}^{\sigma_{n}}\left(q_{i}\right) b\left(q_{m}\right) B_{m-1}^{i}\left(\underline{q}^{i}\right) \psi_{P, n},
$$


where we also separated the $i=m$ contribution to the sum on the r.h.s. of (D.13). Now we pick some $k_{1}, \ldots, k_{m} \in K$, where $K$ is compact and $0 \notin K$, write $A_{\underline{q}}\left(q_{m}\right)=A_{\underline{k}}\left(k_{m}\right)+\left(A_{\underline{q}}\left(q_{m}\right)-A_{\underline{k}}\left(k_{m}\right)\right)$ and obtain from (D.21):

$$
\begin{aligned}
A_{\underline{k}}\left(k_{m}\right) b\left(q_{m}\right) B_{m-1}(\underline{q}) \psi_{P, n}= & v_{\bar{\alpha}}^{\sigma}\left(q_{m}\right) B_{m-1}(\underline{q}) \psi_{P, n}+\sum_{i=1}^{m-1} v_{\bar{\alpha}}^{\sigma_{n}}\left(q_{i}\right) b\left(q_{m}\right) B_{m-1}^{i}\left(\underline{q^{i}}\right) \psi_{P, n} \\
& +\left(A_{\underline{k}}\left(k_{m}\right)-A_{\underline{q}}\left(q_{m}\right)\right) b\left(q_{m}\right) B_{m-1}(\underline{q}) \psi_{P, n} .
\end{aligned}
$$

Next we divide both sides of (D.22) by $A_{k}\left(k_{m}\right)$, which is legitimate as it does not involve any smearing variables $q_{i}, i=1, \ldots, m$ and, by part (a) of the lemma, $\left\|A_{\underline{k}}\left(k_{m}\right)^{-1}\right\| \leq c\left|k_{m}\right|^{-1}$. This gives:

$$
\begin{aligned}
b\left(q_{m}\right) B_{m-1}(\underline{q}) \psi_{P, n}=A_{\underline{k}}\left(k_{m}\right)^{-1} v_{\bar{\alpha}}^{\sigma}\left(q_{m}\right) B_{m-1}(\underline{q}) \psi_{P, n}+A_{\underline{k}}\left(k_{m}\right)^{-1} \sum_{i=1}^{m-1} v_{\bar{\alpha}}^{\sigma_{n}}\left(q_{i}\right) b\left(q_{m}\right) B_{m-1}^{i}\left(\underline{q^{i}}\right) \psi_{P, n} \\
+A_{\underline{k}}\left(k_{m}\right)^{-1}\left(A_{\underline{k}}\left(k_{m}\right)-A_{\underline{q}}\left(q_{m}\right)\right) b\left(q_{m}\right) B_{m-1}(\underline{q}) \psi_{P, n} .
\end{aligned}
$$

To proceed, we introduce the smearing function $\eta_{\underline{k}, k_{m}}^{\varepsilon}\left(q_{m}, \ldots, q_{1}\right):=\eta_{k_{m}}^{\varepsilon}\left(q_{m}\right) \ldots \eta_{k_{1}}^{\varepsilon}\left(q_{1}\right)$ and set

$$
\begin{aligned}
& B_{m-1}^{\varepsilon}(\underline{k}):=b\left(\eta_{k_{m-1}}^{\varepsilon}\right) \ldots b\left(\eta_{k_{1}}^{\varepsilon}\right), \\
& B_{m-1}^{\varepsilon, i}\left(\underline{k}^{i}\right):=b\left(\eta_{k_{m-1}}^{\varepsilon}\right) \ldots \check{i} \ldots b\left(\eta_{k_{1}}^{\varepsilon}\right), \\
& B_{m-1}^{\varepsilon, i, i^{\prime}}\left(\underline{k}^{i, i^{\prime}}\right):=b\left(\eta_{k_{m-1}}^{\varepsilon}\right) \ldots \check{i} \ldots \check{i^{\prime}} \ldots b\left(\eta_{k_{1}}^{\varepsilon}\right) .
\end{aligned}
$$

By smearing with the respective test-functions we obtain from (D.23)-(D.24)

$$
\begin{aligned}
b\left(\eta_{k_{m}}^{\varepsilon}\right) B_{m-1}^{\varepsilon}(\underline{k}) \psi_{P, n}= & A_{\underline{k}}\left(k_{m}\right)^{-1}\left\langle\eta_{k_{m}}^{\varepsilon}, v_{\bar{\alpha}}^{\sigma}\right\rangle B_{m-1}^{\varepsilon}(\underline{k}) \psi_{P, n}+A_{\underline{k}}\left(k_{m}\right)^{-1} \sum_{i=1}^{m-1}\left\langle\eta_{k_{i}}^{\varepsilon}, v_{\bar{\alpha}}^{\sigma_{n}}\right\rangle b\left(\eta_{k_{m}}^{\varepsilon}\right) B_{m-1}^{\varepsilon, i}\left(\underline{k}^{i}\right) \psi_{P, n} \\
& \left.+\int d^{3(m-1)} \underline{q} d^{3} q_{m} \eta_{\underline{k}, k_{m}}^{\varepsilon} \underline{q}, q_{m}\right) A_{\underline{k}}\left(k_{m}\right)^{-1}\left(A_{\underline{k}}\left(k_{m}\right)-A_{\underline{q}}\left(q_{m}\right)\right) b\left(q_{m}\right) B_{m-1}(\underline{q}) \psi_{P, n} .
\end{aligned}
$$

Analysis of (D.28). This is the leading term and we will show that it converges as $\varepsilon \rightarrow 0$ to the expression on the r.h.s. of (D.5). To close the inductive argument we also need to show a uniform bound analogous to (D.10).

Since $k_{m} \neq 0$, the first part of the lemma gives ||$A_{\underline{k}}\left(k_{m}\right)^{-1} \| \leq c\left|k_{m}\right|^{-1}<\infty$. Furthermore, introducing for future reference some continuous functions $g_{i}$, we have

$$
\left\langle g_{i} \eta_{k_{i}}^{\varepsilon}, v_{\bar{\alpha}}^{\sigma}\right\rangle=\int d^{3} q \eta(q) g_{i}\left(k_{i}+\varepsilon q\right) \frac{\chi_{\left[\sigma_{n}, k\right)}\left(k_{i}+\varepsilon q\right)\left|k_{i}+\varepsilon q\right|^{\bar{\alpha}}}{\sqrt{2\left|k_{i}+\varepsilon q\right|}},
$$

hence

$$
\lim _{\varepsilon \rightarrow 0}\left\langle g_{i} \eta_{k_{i}}^{\varepsilon}, v_{\bar{\alpha}}^{\sigma}\right\rangle=g_{i}\left(k_{i}\right) v_{\bar{\alpha}}^{\sigma}\left(k_{i}\right) \quad \text { and } \quad\left|\left\langle g_{i} \eta_{k_{i}}^{\varepsilon}, v_{\bar{\alpha}}^{\sigma}\right\rangle\right| \leq c_{K} \sup _{q \in \operatorname{supp} \eta}\left|g_{i}\left(k_{i}+\varepsilon q\right)\right| \leq c_{K}^{\prime} .
$$

The first relation above is the only place in the proof where the additional assumption $\left|k_{i}\right| \neq \sigma_{n}$ enters to ensure the continuity of the integrand in $\varepsilon$ needed for a dominated convergence argument. (See the last paragraph of the proof for the case $\left|k_{i}\right|=\sigma_{n}$ ). The last bound in (D.31) holds uniformly in $k_{i} \in K$ and in $\varepsilon$ s.t. $\left|k_{i}+\varepsilon q\right|>\frac{1}{2}\left|k_{i}\right|$ for all $q \in \operatorname{supp} \eta$. (The constant $c_{K}$ does not depend on $n, \bar{\alpha}$. The constant $c_{K}^{\prime}$ does depend on $g_{i}$ ). Next, by a-(b) we have $\psi_{P, n} \in D\left(b\left(k_{1}\right) \ldots b\left(k_{m^{\prime}}\right)\right)$. This gives the existence of the following limits

$$
\begin{aligned}
\lim _{\varepsilon \rightarrow 0} B_{m-1}^{\varepsilon}(\underline{k}) \psi_{P, n} & =\left[b\left(k_{1}\right) \ldots b\left(k_{m-1}\right) \psi_{P, n}\right], \\
\lim _{\varepsilon \rightarrow 0} b\left(\eta_{k_{m}}^{\varepsilon}\right) B_{m-1}^{\varepsilon, i}\left(\underline{k}^{i}\right) \psi_{P, n} & =\left[b\left(k_{1}\right) \ldots \check{i} \ldots b\left(k_{m}\right) \psi_{P, n}\right] .
\end{aligned}
$$


Furthermore, estimate (D.10) gives

$$
\left\|B_{m-1}^{\varepsilon}(\underline{k}) \psi_{P, n}\right\| \leq C_{K, m-1}, \quad\left\|b\left(\eta_{k_{m}}^{\varepsilon}\right) B_{m-1}^{\varepsilon, i}\left(\underline{k}^{i}\right) \psi_{P, n}\right\| \leq C_{K, m-1},
$$

uniformly in $k_{1} \ldots, k_{m} \in K$ and $\varepsilon \in\left(0, \varepsilon_{K}\right)$ for some $\varepsilon_{K}>0$. Thus (D.30)-(D.34) give

$$
\begin{aligned}
& \lim _{\varepsilon \rightarrow 0}(\text { r.h.s. }(D .28))=\frac{1}{E_{P, n}-|\underline{k}|_{m}-H_{P-\underline{k}_{m}, n}} \sum_{i=1}^{m} v_{\bar{\alpha}}^{\sigma_{n}}\left(k_{i}\right)\left[b\left(k_{1}\right) \ldots \check{i} \ldots b\left(k_{m}\right) \psi_{P, n}\right]=(\text { r.h.s. }(D .5)), \\
& \|(\text { r.h.s. }(D .28)) \| \leq C_{K, m}^{\prime},
\end{aligned}
$$

uniformly in $k_{1} \ldots, k_{m} \in K$ and $\varepsilon \in\left(0, \varepsilon_{K}^{\prime}\right)$ for some $\varepsilon_{K}^{\prime}>0$. This concludes our analysis of (D.28).

Analysis of (D.29). This is an error term and we are aiming at an estimate

$$
\|(D .29)\| \leq \varepsilon^{1 / 2} C_{K, m},
$$

uniformly in $k_{1}, \ldots, k_{m} \in K$ and $\varepsilon \in\left(0, \varepsilon_{K}\right)$ for some $\varepsilon_{K}>0$. This will give $\lim _{\varepsilon \rightarrow 0}\|(D .29)\|=0$ together with the uniformity in $k_{i}$ and $\varepsilon$ needed to close the inductive argument.

To this end, we note that

$$
\begin{aligned}
& \left(A_{\underline{k}}\left(k_{m}\right)-A_{\underline{q}}\left(q_{m}\right)\right)=L_{\underline{k}, k_{m}}\left(\underline{q}, q_{m}\right)-\left(P-P_{\mathrm{f}}\right) \cdot M_{\underline{k}, k_{m}}\left(\underline{q}, q_{m}\right), \quad \text { where } \\
& L_{\underline{k}, k_{m}}\left(\underline{q}, q_{m}\right):=\sum_{j=1}^{m}\left\{\left(\left|k_{j}\right|-\left|q_{j}\right|\right)+\left(k_{j}-q_{j}\right) \cdot \sum_{j^{\prime}=1}^{m}\left(k_{j^{\prime}}+q_{j^{\prime}}\right)\right\}, \quad M_{\underline{k}, k_{m}}\left(\underline{q}, q_{m}\right):=\sum_{j=1}^{m}\left(k_{j}-q_{j}\right) .
\end{aligned}
$$

The key property of the expressions $L_{\underline{k}, k_{m}}\left(\underline{q}, q_{m}\right)$ and $M_{\underline{k}, k_{m}}\left(\underline{q}, q_{m}\right)$ is that they vanish when all $q_{i} \rightarrow k_{i}$ and therefore

$$
\left.\eta_{\underline{k}, k_{m}}^{\varepsilon}\left(\underline{q}, q_{m}\right) L_{\underline{k}, k_{m}}\left(\underline{q}, q_{m}\right) \rightarrow 0, \quad \eta_{\underline{k}, k_{m}}^{\varepsilon} \underline{(q}, q_{m}\right) M_{\underline{k}, k_{m}}\left(\underline{q}, q_{m}\right) \rightarrow 0 \quad \text { for } \quad \varepsilon \rightarrow 0,
$$

in a sense to be specified below. This mechanism will be used to establish (D.37).

Before exploiting the above observation, we rewrite (D.29) making use of (D.23)-(D.24):

$$
\begin{aligned}
& \left.\int d^{3(m-1)} \underline{q} d^{3} q_{m} \eta_{\underline{k}, k_{m}}^{\varepsilon} \underline{q}, q_{m}\right) A_{\underline{k}}\left(k_{m}\right)^{-1}\left(A_{\underline{k}}\left(k_{m}\right)-A_{\underline{q}}\left(q_{m}\right)\right) b\left(q_{m}\right) B_{m-1}(\underline{q}) \psi_{P, n} \\
= & \left.\int d^{3(m-1)} \underline{q} d^{3} q_{m} \eta_{\underline{k}, k_{m}}^{\varepsilon} \underline{q}, q_{m}\right) A_{\underline{k}}\left(k_{m}\right)^{-1}\left(A_{\underline{k}}\left(k_{m}\right)-A_{\underline{q}}\left(q_{m}\right)\right) A_{\underline{k}}\left(k_{m}\right)^{-1} v_{\bar{\alpha}}^{\sigma}\left(q_{m}\right) B_{m-1}(\underline{q}) \psi_{P, n} \\
+ & \left.\int d^{3(m-1)} \underline{q} d^{3} q_{m} \eta_{\underline{k}, k_{m}}^{\varepsilon} \underline{q}, q_{m}\right) A_{\underline{k}}\left(k_{m}\right)^{-1}\left(A_{\underline{k}}\left(k_{m}\right)-A_{\underline{q}}\left(q_{m}\right)\right) A_{\underline{k}}\left(k_{m}\right)^{-1} \sum_{i=1}^{m-1} v_{\bar{\alpha}}^{\sigma}\left(q_{i}\right) b\left(q_{m}\right) B_{m-1}^{i}\left(\underline{q}^{i}\right) \psi_{P, n} \\
+ & \left.\int d^{3(m-1)} \underline{q} d^{3} q_{m} \eta_{\underline{k}, k_{m}}^{\varepsilon} \underline{q}, q_{m}\right)\left\{A_{\underline{k}}\left(k_{m}\right)^{-1}\left(A_{\underline{k}}\left(k_{m}\right)-A_{\underline{q}}\left(q_{m}\right)\right)\right\}^{2} b\left(q_{m}\right) B_{m-1}(q) \psi_{P, n},
\end{aligned}
$$

and we will show that (D.41), (D.42), (D.43) satisfy bounds of the form (D.37).

Each contribution (D.41) and (D.42) can be divided into two parts coming from the two terms on the r.h.s. of (D.38), which we denote $(\ldots)_{L}$ and $(\ldots)_{M}$. We will consider only the $M$-parts as the analysis of $L$-parts is analogous. Since

$$
\left\|A_{\underline{k}}\left(k_{m}\right)^{-1}\right\| \leq C_{K}, \quad \sum_{\ell=1,2,3}\left\|A_{\underline{k}}\left(k_{m}\right)^{-1}\left(P-P_{\mathrm{f}}\right)^{\ell}\right\| \leq C_{K},
$$


where $\ell$ is a vector index, we can write

$$
\begin{aligned}
\left\|(D .41)_{M}\right\| \leq & C_{K} \sup _{\ell=1,2,3}\left\|\int d^{3(m-1)} \underline{q} d^{3} q_{m} \eta_{\underline{k}, k_{m}}^{\varepsilon}\left(\underline{q}, q_{m}\right) M_{\underline{k}, k_{m}}^{\ell}\left(\underline{q}, q_{m}\right) v_{\bar{\alpha}}^{\sigma}\left(q_{m}\right) B_{m-1}(\underline{q}) \psi_{P, n}\right\| \\
\leq & C_{K} \sup _{\ell=1,2,3}\left|\left\langle\left(k_{m}-q_{m}\right)^{\ell} \eta_{k_{m}}^{\varepsilon}, v_{\bar{\alpha}}^{\sigma}\right\rangle\right|\left\|B_{m-1}^{\varepsilon}(\underline{k}) \psi_{P, n}\right\| \\
& +C_{K} \mid\left\langle\eta_{k_{m}}^{\varepsilon}, v_{\bar{\alpha}}^{\sigma}\right\rangle \sup _{\ell=1,2,3} \sum_{j=1}^{m-1}\left\|b\left(\left(k_{j}-q_{j}\right)^{\ell} \eta_{k_{j}}^{\varepsilon}\right) B_{m-1}^{\varepsilon, j}\left(\underline{k}^{j}\right) \psi_{P, n}\right\| .
\end{aligned}
$$

By the second relation in (D.31) with $i=m$ and $g_{m}(q):=\left(k_{m}-q\right)$, we have

$$
\left|\left\langle\left(k_{m}-q_{m}\right)^{\ell} \eta_{k_{m}}^{\varepsilon}, v_{\bar{\alpha}}^{\sigma}\right\rangle\right| \leq c_{K} \varepsilon, \quad\left|\left\langle\eta_{k_{m}}^{\varepsilon}, v_{\bar{\alpha}}^{\sigma}\right\rangle\right| \leq c_{K},
$$

where the latter relation corresponds to $g_{m} \equiv 1$. Furthermore, by the first estimate in (D.10) we can write

$$
\left\|b\left(\left(k_{j}-q_{j}\right)^{\ell} \eta_{k_{j}}^{\varepsilon}\right) B_{m-1}^{\varepsilon, j}\left(\underline{k}^{j}\right) \psi_{P, n}\right\| \leq \varepsilon C_{K}, \quad\left\|B_{m-1}^{\varepsilon}(\underline{k}) \psi_{P, n}\right\| \leq C_{K} .
$$

Thus altogether

$$
\left\|(D .41)_{M}\right\| \leq \varepsilon C_{K}^{\prime} .
$$

Next, we note that each term in the sum in (D.42) differs from (D.41) only by renaming the indices $i \leftrightarrow m$. Thus by analogous arguments we have

$$
\left\|(D .42)_{M}\right\| \leq \varepsilon C_{K}^{\prime} \quad \text { and altogether } \quad\|(D .41)+(D .42)\| \leq \varepsilon C_{K}^{\prime \prime} .
$$

Now we consider (D.43). This contribution can be divided into four parts coming from the two terms on the r.h.s. of (D.38). We will denote them $(D .43)_{L L},(D .43)_{L M},(D .43)_{M L},(D .43)_{M M}$. As they all have very similar structure, it suffices to consider (D.43) MM . Making use again of (D.44), we obtain

$$
\begin{aligned}
& \left\|(D .43)_{M M}\right\| \\
& \left.\left.\leq C_{K} \sup _{\ell, \ell^{\prime}=1,2,3} \| A_{\underline{k}}\left(k_{m}\right)^{-1}\left(P-P_{\mathrm{f}}\right)^{\ell} \int d^{3(m-1)} \underline{q} d^{3} q_{m} \eta_{\underline{k}, k_{m}}^{\varepsilon} \underline{q}, q_{m}\right) M_{\underline{k}, k_{m}}^{\ell} \underline{q}, q_{m}\right) M_{\underline{k}, k_{m}}^{\ell^{\prime}}\left(\underline{q}, q_{m}\right) b\left(q_{m}\right) B_{m-1}(\underline{q}) \psi_{P, n} \| \\
& \leq C_{K} \sup _{\ell, \ell^{\prime}=1,2,3}\left\|A_{\underline{k}}\left(k_{m}\right)^{-1}\left(P-P_{\mathrm{f}}\right)^{\ell} b\left(\left(k_{m}-q_{m}\right)^{\ell}\left(k_{m}-q_{m}\right)^{\ell^{\prime}} \eta_{k_{m}}^{\varepsilon}\right) B_{m-1}^{\varepsilon}(\underline{k}) \psi_{P, n}\right\| \\
& +C_{K} \sup _{\ell, \ell^{\prime}=1,2,3} \sum_{j=1}^{m-1}\left\|A_{\underline{k}}\left(k_{m}\right)^{-1}\left(P-P_{\mathrm{f}}\right)^{\ell} b\left(\left(k_{m}-q_{m}\right)^{\ell} \eta_{k_{m}}^{\varepsilon}\right) b\left(\left(k_{j}-q_{j}\right)^{\ell^{\prime}} \eta_{k_{j}}^{\varepsilon}\right) B_{m-1}^{\varepsilon, j}\left(\underline{k}^{j}\right) \psi_{P, n}\right\| \\
& +C_{K} \sup _{\ell, \ell^{\prime}=1,2,3} \sum_{j=1}^{m-1}\left\|A_{\underline{k}}\left(k_{m}\right)^{-1}\left(P-P_{\mathrm{f}}\right)^{\ell} b\left(\eta_{k_{m}}^{\varepsilon}\right) b\left(\left(k_{j}-q_{j}\right)^{\ell}\left(k_{j}-q_{j}\right)^{\ell^{\prime}} \eta_{k_{j}}^{\varepsilon}\right) B_{m-1}^{\varepsilon, j}\left(\underline{k}^{j}\right) \psi_{P, n}\right\| \\
& +C_{K} \sup _{\ell, \ell^{\prime}=1,2,3} \sum_{\substack{j, j^{\prime}=1 \\
j \neq j^{\prime}}}^{m-1}\left\|A_{\underline{k}}\left(k_{m}\right)^{-1}\left(P-P_{\mathrm{f}}\right)^{\ell} b\left(\eta_{k_{m}}^{\varepsilon}\right) b\left(\left(k_{j}-q_{j}\right)^{\ell} \eta_{k_{j}}^{\varepsilon}\right) b\left(\left(k_{j^{\prime}}-q_{j^{\prime}}\right)^{\ell^{\prime}} \eta_{k_{j^{\prime}}}^{\varepsilon}\right) B_{m-1}^{\varepsilon, j, j^{\prime}}\left(\underline{k}^{j, j^{\prime}}\right) \psi_{P, n}\right\| .
\end{aligned}
$$

To analyze the above expressions we make the following observations: It is an easy application of Lemmas A.1 and A.3 that

$$
\left\|A_{\underline{k}}\left(k_{m}\right)^{-1}\left(P-P_{\mathrm{f}}\right)^{\ell} H_{\mathrm{f}}^{1 / 2}\right\| \leq c_{K} .
$$


Consequently, using again Lemma A.1, we obtain

$$
\begin{aligned}
\| A_{\underline{k}}\left(k_{m}\right)^{-1}\left(P-P_{\mathrm{f}}\right)^{\ell} b\left(\left(k_{m}\right.\right. & \left.\left.-q_{m}\right)^{\ell}\left(k_{m}-q_{m}\right)^{\ell^{\prime}} \eta_{k_{m}}^{\varepsilon}\right) \| \leq c\left(\int d^{3} q_{m}\left|q_{m}\right|^{-1}\left(k_{m}-q_{m}\right)^{4} \eta_{k_{m}}^{\varepsilon}\left(q_{m}\right)^{2}\right)^{1 / 2} \\
& \leq c^{\prime} \varepsilon^{1 / 2}\left(\int \frac{d^{3} q_{m}}{\varepsilon^{3}}\left|q_{m}\right|^{-1} \frac{\left|q_{m}-k_{m}\right|^{4}}{\varepsilon^{4}} \eta\left(\frac{q_{m}-k_{m}}{\varepsilon}\right)^{2}\right)^{1 / 2} \\
& =c \varepsilon^{1 / 2}\left(\int d^{3} q_{m}^{\prime}\left|\varepsilon q_{m}^{\prime}+k_{m}\right|^{-1}\left|\left(q_{m}^{\prime}\right)^{2} \eta\left(q_{m}^{\prime}\right)\right|^{2}\right)^{1 / 2} \leq c^{\prime} \varepsilon^{1 / 2}\left|k_{m}\right|^{-1 / 2}
\end{aligned}
$$

An analogous consideration gives

$$
\left\|A_{\underline{k}}\left(k_{m}\right)^{-1}\left(P-P_{\mathrm{f}}\right)^{\ell} b\left(\left(k_{m}-q_{m}\right)^{\ell} \eta_{k_{m}}^{\varepsilon}\right)\right\| \leq c^{\prime} \varepsilon^{-1 / 2}\left|k_{m}\right|^{-1 / 2} .
$$

Furthermore, by estimate (D.10),

$$
\left\|B_{m-1}^{\varepsilon, j}\left(\underline{k}^{j}\right) \psi_{P, n}\right\| \leq C_{K, m-1}, \quad\left\|b\left(\left(k_{j}-q_{j}\right)^{\ell^{\prime}} \eta_{k_{j}}^{\varepsilon}\right) B_{m-1}^{\varepsilon, j}\left(\underline{k}^{j}\right) \psi_{P, n}\right\| \leq \varepsilon C_{K, m-1} .
$$

Taking (D.55)-(D.57) into account, we get

$$
\|D .50\| \leq \varepsilon^{1 / 2} C_{K}, \quad\|D .51\| \leq \varepsilon^{1 / 2} C_{K} .
$$

Now we note that (D.52), (D.53) have the same structure as (D.50), (D.51), respectively, and therefore satisfy analogous bounds. Consequently $\left\|(D .43)_{M M}\right\| \leq \varepsilon^{1 / 2} C_{K}$ and such bound holds also for the remaining contributions to (D.43). Together with (D.49), this concludes the proof of (D.37).

We have therefore proven property (b) from the statement of the lemma for $m$. Furthermore, we have verified that

$$
\left\|b\left(\eta_{k_{1}}^{\varepsilon}\right) \ldots b\left(\eta_{k_{m}}^{\varepsilon}\right) \psi_{P, n}\right\| \leq C_{K, m}
$$

uniformly in $k_{1}, \ldots, k_{m} \in K$ and $\varepsilon \in\left(0, \varepsilon_{K}\right)$ for some $\varepsilon_{K}>0$. Therefore, by Lemma D.3 we obtain statement (c) from the lemma for $m$, which concludes the inductive argument under the additional assumption that $\left|k_{i}\right| \neq \sigma_{n}$, $i=1, \ldots, m$.

Let us now explain how to drop this assumption at a cost of providing factors $\frac{1}{2}$ in formula (D.5): It suffices to reestablish the first relation in (D.31) in the case $\left|k_{i}\right|=\sigma_{n}$. We have, making use of (D.30), and assuming that $\varepsilon$ is sufficiently small so that $0<\left|k_{i}+\varepsilon q\right| \ll \kappa$ for $q \in \operatorname{supp} \eta$,

$$
\left\langle g_{i} \eta_{k_{i}}^{\varepsilon}, v_{\bar{\alpha}}^{\sigma}\right\rangle=\int d^{3} q \eta(q) g_{i}\left(k_{i}+\varepsilon q\right) \frac{\chi_{\left[\sigma_{n}, \kappa\right)}\left(k_{i}+\varepsilon q\right)\left|k_{i}+\varepsilon q\right|^{\bar{\alpha}}}{\sqrt{2\left|k_{i}+\varepsilon q\right|}}=g\left(k_{i}\right) \frac{\left|k_{i}\right|^{\bar{\alpha}}}{\sqrt{2\left|k_{i}\right|}} \int_{\sigma_{n} \leq\left|k_{i}+\varepsilon q\right|} d^{3} q \eta(q)+O(\varepsilon),
$$

where $O(\varepsilon)$ denotes an error term which tends to zero as $\varepsilon \rightarrow 0$ and we made use of continuity of $k \mapsto g(k) \frac{\mid k k^{\bar{\alpha}}}{\sqrt{2|k|}}$ near $k_{i} \neq 0$. Now by squaring both sides of the relation $\sigma_{n} \leq\left|k_{i}+\varepsilon q\right|$ and using $\left|k_{i}\right|^{2}=\sigma_{n}^{2}$ we get

$$
\cos \theta \geq-\frac{\varepsilon|q|}{2\left|k_{i}\right|},
$$

where $\theta$ is the angle between $k_{i}$ and $q$. Exploiting the spherical symmetry of $\eta$ to choose the third axis of the reference frame in the direction of $k_{i}$ and passing to spherical coordinates, we have

$$
\begin{aligned}
\int_{\sigma_{n} \leq\left|k_{i}+\varepsilon q\right|} d^{3} q \eta(q) & =2 \pi \int|q|^{2} d|q| \eta(|q|) \int_{\cos \theta \geq-\frac{\varepsilon|q|}{2 k_{i} \mid}} d \cos \theta \\
& =2 \pi \int|q|^{2} d|q| \eta(|q|)\left(1+\frac{\varepsilon|q|}{2\left|k_{i}\right|}\right) \\
& =\frac{1}{2} \int d^{3} q \eta(q)+O(\varepsilon) .
\end{aligned}
$$


Since $\int d^{3} q \eta(q)=1$, this concludes the argument.

Lemma D.3. Let $f_{i} \in L^{1}\left(\mathbb{R}^{3}\right) \cap L^{2}\left(\mathbb{R}^{3}\right), i=1, \ldots . . m$, be compactly supported outside of zero and let $K$ be a compact set, not containing zero, whose interior contains all supp $f_{i}$. Suppose that $\psi \in D\left(b\left(k_{1}\right) \ldots b\left(k_{m}\right)\right)$ for all $k_{i} \in K$. Suppose furthermore that

$$
\left\|b\left(\eta_{k_{1}}^{\varepsilon}\right) \ldots b\left(\eta_{k_{m}}^{\varepsilon}\right) \psi\right\| \leq C_{K, m}
$$

uniformly in $k_{1}, \ldots, k_{m} \in K$ and $\varepsilon \in\left(0, \varepsilon_{K}\right)$ for some $\varepsilon_{K}>0$. Then $K^{\times m} \ni\left(k_{1}, \ldots, k_{m}\right) \rightarrow\left[b\left(k_{1}\right) \ldots b\left(k_{m}\right) \psi\right]$ is weakly measurable and

$$
\int d^{3} k_{1} \ldots d^{3} k_{m} \bar{f}_{1}\left(k_{1}\right) \ldots \bar{f}_{m}\left(k_{m}\right)\left[b\left(k_{1}\right) \ldots b\left(k_{m}\right) \psi\right]=b\left(f_{1}\right) \ldots b\left(f_{m}\right) \psi
$$

where the l.h.s. is a weak integral and the r.h.s. is the usual definition.

Remark D.4. We prove this lemma for functions from $L^{1}\left(\mathbb{R}^{3}\right) \cap L^{2}\left(\mathbb{R}^{3}\right)$ in order to accommodate $h_{n+1}$ from the proof of Lemma D.5. For the assumption (D.63) see the proof of Lemma D.2.

Proof. First we note that by iterating (A.4), we obtain for any functions $f_{1}, \ldots, f_{m}$ as in the statement of the lemma and $\psi \in C^{\infty}\left(H_{P, \text { free }}\right)$

$$
\left\|b\left(f_{1}\right) \ldots b\left(f_{m}\right) \psi\right\| \leq c_{m}(\psi)\left\|f_{1}\right\|_{2} \ldots\left\|f_{m}\right\|_{2}
$$

where the constant $c_{m}(\psi)$ depends on $\psi$ and we used that $f_{i}$ are supported away from zero. Now let us set $\underline{k}=\left(k_{1}, \ldots, k_{m}\right)$ and write $F(\underline{k}):=f_{1}\left(k_{1}\right) \ldots f_{m}\left(k_{m}\right)$. By standard density arguments, for any $\theta>0$ we can find a function $F_{\theta}(\underline{k}):=f_{1, \theta}\left(k_{1}\right) \ldots f_{m, \theta}\left(k_{m}\right)$, with $f_{i, \theta} \in C_{0}^{\infty}\left(\mathbb{R}^{3 m}\right)$, s.t.

$$
\left\|f_{i}-f_{i, \theta}\right\|_{2} \leq \theta .
$$

Furthermore, we can ensure that all $f_{i, \theta}$ are supported in the compact set $K \subset \mathbb{R}^{3} \backslash\{0\}$ from the statement of the lemma. Then we also have

$$
\left\|f_{i}-f_{i, \theta}\right\|_{1} \leq|K|^{1 / 2} \theta^{1 / 2}
$$

where $|K|$ is the volume of $K$. Using (D.65), (D.66), we obtain

$$
\left\|\left(b\left(f_{1}\right) \ldots b\left(f_{m}\right)\right) \psi-\left(b\left(f_{\theta, 1}\right) \ldots b\left(f_{\theta, m}\right)\right) \psi\right\| \leq C_{1} \theta .
$$

Next, we note that for any $\psi^{\prime} \in \mathcal{F}$ and any fixed $\varepsilon \in\left(0, \varepsilon_{K}\right]$ the function given by $K^{\times m} \ni\left(k_{1}, \ldots, k_{m}\right) \mapsto$ $\left\langle\psi^{\prime}, b\left(\eta_{k_{1}}^{\varepsilon}\right) \ldots b\left(\eta_{k_{m}}^{\varepsilon}\right) \psi\right\rangle$ is measurable. This is easily seen by expressing $\psi, \psi^{\prime}$ in terms of their Fock space components and noting that the resulting sum of measurable functions converges for any $\left(k_{1}, \ldots, k_{m}\right) \in K^{\times m}$. Similarly, since $\psi \in D\left(b\left(k_{1}\right) \ldots b\left(k_{m}\right)\right)$, we obtain that $\left\langle\psi^{\prime},\left[b\left(k_{1}\right) \ldots b\left(k_{m}\right) \psi\right]\right\rangle=\lim _{\varepsilon \rightarrow 0}\left\langle\psi^{\prime}, b\left(\eta_{k_{1}}^{\varepsilon}\right) \ldots b\left(\eta_{k_{m}}^{\varepsilon}\right) \psi\right\rangle$ is a measurable function. Moreover, using the boundedness assumption (D.63), the dominated convergence theorem and estimate in (D.67), we can write

$$
\begin{array}{r}
\left|\int d^{3 m} \underline{k}\left(\bar{F}(\underline{k})-\bar{F}_{\theta}(\underline{k})\right)\left\langle\psi^{\prime},\left[b\left(k_{1}\right) \ldots b\left(k_{m}\right) \psi\right]\right\rangle\right|=\lim _{\varepsilon \rightarrow 0}\left|\int d^{3 m} \underline{k}\left(\bar{F}(\underline{k})-\bar{F}_{\theta}(\underline{k})\right)\left\langle\psi^{\prime}, b\left(\eta_{k_{1}}^{\varepsilon}\right) \ldots b\left(\eta_{k_{m}}^{\varepsilon}\right) \psi\right\rangle\right| \\
\leq C_{K, m}\left\|\psi^{\prime}\right\| \sum_{i=1}^{m}\left(\left\|f_{1}\right\|_{1} \ldots\left\|f_{i-1}\right\|_{1} K^{1 / 2} \theta^{1 / 2}\left\|f_{i+1, \theta}\right\|_{1} \ldots\left\|f_{m, \theta}\right\|_{1}\right) . \text { (D.69) }
\end{array}
$$


In view of the estimates (D.68) and (D.69) it suffices to prove (D.64) for $f_{i, \theta}$. For this purpose, by (D.65) and the fact that $f_{i, \theta}$ are supported away from zero, we get for sufficiently small $\varepsilon$

$$
\begin{aligned}
& \left\|\int d^{3 m} \underline{k} \bar{f}_{1, \theta}\left(k_{1}\right) \ldots \bar{f}_{m, \theta}\left(k_{m}\right)\left(b\left(\eta_{k_{1}}^{\varepsilon}\right) \ldots b\left(\eta_{k_{m}}^{\varepsilon}\right)\right) \psi-\left(b\left(f_{1, \theta}\right) \ldots b\left(f_{m, \theta}\right)\right) \psi\right\| \\
\leq & c_{m} \sum_{i=1}^{m}\left(\left\|\left(\eta_{(\cdot)}^{\varepsilon} * f_{1, \theta}\right)\right\|_{2} \ldots\left\|\left(\eta_{(\cdot)}^{\varepsilon} * f_{i-1, \theta}\right)\right\|_{2}\right)\left\|\left(\eta_{(\cdot)}^{\varepsilon} * f_{i, \theta}\right)-f_{i, \theta}\right\|_{2}\left(\left\|f_{i+1, \theta}\right\|_{2} \ldots\left\|f_{m, \theta}\right\|_{2}\right) .
\end{aligned}
$$

Now we analyse the relevant factor

$$
\begin{aligned}
\left(\eta_{(\cdot)}^{\varepsilon} * f_{i, \theta}\right)\left(k^{\prime}\right)-f\left(k^{\prime}\right) & =\int d^{3} k \varepsilon^{-3} \eta\left(\left(k^{\prime}-k\right) \varepsilon^{-1}\right) f_{i, \theta}(k)-f_{i, \theta}\left(k^{\prime}\right) \\
& =\int d^{3} k \eta(k)\left(f_{i, \theta}\left(k^{\prime}-\varepsilon k\right)-f_{i, \theta}\left(k^{\prime}\right)\right) \\
& =-\varepsilon \int_{0}^{1} d \lambda \int d^{3} k \eta(k) \nabla f_{i, \theta}\left(k^{\prime}-\varepsilon \lambda k\right) \cdot k
\end{aligned}
$$

By substituting (D.71) to (D.70), making use of

$$
\left|\int_{0}^{1} d \lambda \int d^{3} k \eta(k) \nabla f_{i, \theta}\left(k^{\prime}-\varepsilon \lambda k\right) \cdot k\right|^{2} \leq c \int_{0}^{1} d \lambda \int d^{3} k\left|\eta(k) \nabla f_{i, \theta}\left(k^{\prime}-\varepsilon \lambda k\right) \cdot k\right|^{2}
$$

(which follows from the compactness of the region of integration in $k$ and $\lambda$ ), the r.h.s of (D.70) tends to zero as $\epsilon \rightarrow 0$ for any fixed $\theta$.

In view of the above lemma we will simply write $b(k) \psi$ for $[b(k) \psi]$. For the proof of Theorem 2.1 we only need Lemma D. 2 for $m=1$, but the case $m \geq 1$ is needed in Theorem 2.2 which we prove in [15].

Lemma D.5. [29] Let $|P| \leq P_{\max }=1 / 3$ and $|\lambda| \in\left(0, \lambda_{0}\right]$. Then

$$
\begin{aligned}
\left\|\left(W_{P, n+1} \widetilde{W}_{P, n+1}^{*}-1\right) \hat{\phi}_{P, n+1}\right\| & \leq c|\lambda|\left|\nabla E_{P, n+1}-\nabla E_{P, n}\right|\left|\ln \sigma_{n+1}\right|\left\|\hat{\phi}_{P, n+1}\right\|, \\
\left\|\left(W_{P, n+1} W_{P, n}^{*}-1\right) \hat{\phi}_{P, n+1}\right\| & \leq c|\lambda|\left|\nabla E_{P, n+1}-\nabla E_{P, n}\right|\left|\ln \sigma_{n+1}\right|\left\|\hat{\phi}_{P, n+1}\right\|+c|\lambda|(\bar{\alpha})^{-1} \sigma_{n}^{\bar{\alpha}}\left\|\hat{\phi}_{P, n+1}\right\|,
\end{aligned}
$$

where (only) for (D.74) we assumed $\bar{\alpha}>0$ and both estimates also hold after replacing $\hat{\phi}_{P, n+1}$ with $\phi_{P, n+1}$.

Proof. We will prove the two estimates in parallel. Let us set

$$
\begin{aligned}
& \tilde{f}_{n+1}^{\varepsilon}(k):=\lambda \chi_{\left[\sigma_{n+1}, \kappa\right)}(k)|k|^{\bar{\alpha}} \frac{1}{\sqrt{2}|k|^{\frac{3}{2}} \alpha_{P, n}(\hat{k})}-\varepsilon \lambda \chi_{\left[\sigma_{n+1}, \sigma_{n}\right]}(k)|k|^{\bar{\alpha}} \frac{1}{\sqrt{2}|k|^{\frac{3}{2}} \alpha_{P, n}(\hat{k})}, \\
& f_{n+1}(k):=\lambda \chi_{\left[\sigma_{n+1}, \kappa\right)}(k)|k|^{\bar{\alpha}} \frac{1}{\sqrt{2}|k|^{\frac{3}{2}} \alpha_{P, n+1}(\hat{k})},
\end{aligned}
$$

where $\varepsilon \in\{0,1\}$ and define $h_{n+1}^{\varepsilon}:=f_{n+1}-\tilde{f}_{n+1}^{\varepsilon}$. We estimate using spectral calculus

$$
\begin{aligned}
\left\|\left(e^{\left(b^{*}\left(f_{n+1}\right)-b\left(f_{n+1}\right)\right)} e^{-\left(b^{*}\left(\tilde{f}_{n+1}^{\varepsilon}\right)-b\left(\tilde{f}_{n+1}^{\varepsilon}\right)\right)}-1\right) \hat{\phi}_{P, n+1}\right\| & \leq c\left\|\left(b^{*}\left(h_{n+1}^{\varepsilon}\right)-b\left(h_{n+1}^{\varepsilon}\right)\right) \hat{\phi}_{P, n+1}\right\| \\
& \leq c\left\|h_{n+1}^{\varepsilon}\right\|\left\|\hat{\phi}_{P, n+1}\right\|+c^{\prime}\left\|b\left(h_{n+1}^{\varepsilon}\right) \hat{\phi}_{P, n+1}\right\|,
\end{aligned}
$$

where the case $\varepsilon=0$ (resp. $\varepsilon=1$ ) corresponds to estimate (D.73) (resp. (D.74)). We recall the definitions $\breve{\phi}_{P, n}:=\phi_{P, n} /\left\|\phi_{P, n}\right\|, \phi_{P, n}:=W_{P, n} \widetilde{W}_{P, n}^{*} \hat{\phi}_{P, n}$ (cf. (3.18)) and note that $\breve{\psi}_{P, n}:=W_{P, n}^{*} \breve{\phi}_{P, n}$ differs at most by a complex 
factor from $\psi_{P, n}$ appearing in (D.5). From these relations we get $\hat{\phi}_{P, n+1}=\left\|\hat{\phi}_{P, n+1}\right\| \widetilde{W}_{P, n+1} \breve{\psi}_{P, n+1}$. Next, we set $\hat{\phi}_{P, n+1}^{*}:=\hat{\phi}_{P, n+1} /\left\|\hat{\phi}_{P, n+1}\right\|$ and compute

$$
b\left(h_{n+1}^{\varepsilon}\right) \hat{\phi}_{P, n+1}^{*}=b\left(h_{n+1}^{\varepsilon}\right) \widetilde{W}_{P, n+1} \breve{\psi}_{P, n+1}=\widetilde{W}_{P, n+1}\left(b\left(h_{n+1}^{\varepsilon}\right)+\left\langle h_{n+1}^{\varepsilon}, \tilde{f}_{n+1}^{0}\right\rangle\right) \check{\psi}_{P, n+1} .
$$

Making use of Lemmas D.2, D.5 we estimate the first term on the r.h.s. above

$$
\begin{aligned}
\left\|b\left(h_{n+1}^{\varepsilon}\right) \breve{\psi}_{P, n+1}\right\| \leq & \left\|\int_{\sigma_{n+1}}^{k} d^{3} k h_{n+1}^{0}(k) \frac{\lambda}{\sqrt{2|k|}} \frac{1}{E_{P, n+1}-|k|-H_{P-k, n+1}} \breve{\psi}_{P, n+1}\right\| \\
& +\varepsilon\left\|\int_{\sigma_{n+1}}^{\sigma_{n}} d^{3} k \frac{1}{\alpha_{P, n}(\hat{k})} \frac{\lambda^{2}}{2|k|^{2}} \frac{1}{E_{P, n+1}-|k|-H_{P-k, n+1}} \breve{\psi}_{P, n+1}\right\| \\
\leq & c \lambda^{2} \int_{\sigma_{n+1}}^{k} d^{3} k \frac{1}{2|k|^{3}}\left|\frac{1}{\alpha_{P, n+1}(\hat{k})}-\frac{1}{\alpha_{P, n}(\hat{k})}\right| \mid \breve{\psi}_{P, n+1} \|+c \varepsilon \lambda^{2}(\bar{\alpha})^{-1} \sigma_{n}^{2 \bar{\alpha}} \\
= & c \lambda^{2} \int_{\sigma_{n+1}}^{k} d^{3} k \frac{1}{2|k|^{3}}\left|\frac{\hat{k} \cdot\left(\nabla E_{P, n+1}-\nabla E_{P, n}\right)}{\alpha_{P, n+1}(\hat{k}) \alpha_{P, n}(\hat{k})}\right|+c \varepsilon \lambda^{2}(\bar{\alpha})^{-1} \sigma_{n}^{2 \bar{\alpha}} \\
\leq & c|\lambda|^{2}\left|\nabla E_{P, n+1}-\nabla E_{P, n}\right|\left|\ln \sigma_{n+1}\right|+c \varepsilon \lambda^{2}(\bar{\alpha})^{-1} \sigma_{n}^{2 \bar{\alpha}},
\end{aligned}
$$

where we refer to "Standing assumptions and conventions" in Section 2 for the definitions of $\int_{\sigma_{n+1}}^{K} d^{3} k, \int_{\sigma_{n+1}}^{K} d^{3} k$. Now we consider the second term on the r.h.s. of (D.78):

$$
\begin{aligned}
\left\langle h_{n+1}^{\varepsilon}, \tilde{f}_{n+1}^{0}\right\rangle= & \lambda^{2} \bar{\int}_{\sigma_{n+1}}^{k} d^{3} k\left(\frac{1}{\sqrt{2}|k|^{\frac{3}{2}} \alpha_{P, n}(\hat{k})}-\frac{1}{\sqrt{2}|k|^{\frac{3}{2}} \alpha_{P, n+1}(\hat{k})}\right) \frac{1}{\sqrt{2}|k|^{\frac{3}{2}} \alpha_{P, n}(\hat{k})} \\
& +\varepsilon \lambda^{2} \bar{\int}_{\sigma_{n+1}}^{\sigma_{n}} d^{3} k\left(\frac{1}{\sqrt{2}|k|^{\frac{3}{2}} \alpha_{P, n}(\hat{k})}\right) \frac{1}{\sqrt{2}|k|^{\frac{3}{2}} \alpha_{P, n}(\hat{k})} .
\end{aligned}
$$

This satisfies again a bound of the form (D.79). Finally, we consider the first expression on the r.h.s. of (D.77):

$$
\begin{aligned}
\left\|h_{n+1}^{\varepsilon}\right\| & \leq|\lambda|\left(\int_{\sigma_{n+1}}^{k} d^{3} k\left|\frac{1}{\sqrt{2}|k|^{\frac{3}{2}} \alpha_{P, n}(\hat{k})}-\frac{1}{\sqrt{2}|k|^{\frac{3}{2}} \alpha_{P, n+1}(\hat{k})}\right|^{2}\right)^{1 / 2}+\varepsilon|\lambda|\left(\int_{\sigma_{n+1}}^{\sigma_{n}} d^{3} k\left(\frac{1}{\sqrt{2}|k|^{\frac{3}{2}} \alpha_{P, n}(\hat{k})}\right)^{2}\right)^{1 / 2} \\
& \leq c|\lambda|\left|\nabla E_{P, n+1}-\nabla E_{P, n} \| \ln \sigma_{n+1}\right|^{1 / 2}+c \varepsilon|\lambda|(\bar{\alpha})^{-1 / 2} \sigma_{n}^{\bar{\alpha}}
\end{aligned}
$$

which again satisfies the required bounds. Finally, we note that replacing $\hat{\phi}_{P, n+1}$ with $\phi_{P, n+1}$ amounts to replacing $\tilde{f}_{n+1}^{0}$ with $\tilde{f}_{n+1}^{1}$ on the r.h.s. of (D.78). Consequently, the last term on the r.h.s. of (D.80) can be dropped and the region of integration in the first term restricted to $\left[\sigma_{n}, \kappa\right]$. Clearly the resulting expression satisfies again a bound of the form (D.79).

Lemma D.6. [29] Let $|P| \leq P_{\max }=1 / 3$ and $|\lambda| \in\left(0, \lambda_{0}\right.$ ]. Then

$$
\left|\nabla E_{P, n-1}-\nabla E_{P, n}\right| \leq c_{1}\left[\lambda^{2} \sigma_{n-1}+\left\|\frac{\hat{\phi}_{P, n}}{\left\|\hat{\phi}_{P, n}\right\|}-\frac{\phi_{P, n-1}}{\left\|\phi_{P, n-1}\right\|}\right\|\right] .
$$

Proof. First, we recall the definitions $\check{\psi}_{P, n}:=W_{P, n}^{*} \check{\phi}_{P, n}, \breve{\phi}_{P, n}:=\phi_{P, n} /\left\|\phi_{P, n}\right\|$ and $\phi_{P, n}:=W_{P, n} \widetilde{W}_{P, n}^{*} \hat{\phi}_{P, n}$ (cf. (3.18) and Definition 5.3). Now by a standard computation and definition (3.22)

$$
\begin{aligned}
W_{P, n} P_{\mathrm{f}} W_{P, n}^{*} & =P_{\mathrm{f}}-\lambda \int_{\sigma_{n}}^{k} d^{3} k \frac{k}{\sqrt{2}|k|^{3 / 2} \alpha_{P, n}(\hat{k})}\left(b^{*}(k)+b(k)\right)+\lambda^{2} \bar{\int}_{\sigma_{n}}^{k} d^{3} k \frac{k}{2|k|^{3} \alpha_{P, n}^{2}(\hat{k})} \\
& =\Pi_{P, n}+\lambda^{2} \bar{\int}_{\sigma_{n}}^{k} d^{3} k \frac{k}{2|k|^{3} \alpha_{P, n}^{2}(\hat{k})} .
\end{aligned}
$$


Thus we have by the Hellmann-Feynman theorem and formula (D.83)

$$
\begin{aligned}
\nabla E_{P, n} & =\left\langle\breve{\psi}_{P, n},\left(P-P_{\mathrm{f}}\right) \breve{\psi}_{P, n}\right\rangle=\left\langle W_{P, n} \breve{\psi}_{P, n}, W_{P, n}\left(P-P_{\mathrm{f}}\right) W_{P, n}^{*} W_{P, n} \check{\psi}_{P, n}\right\rangle \\
& =-\left\langle W_{P, n} \check{\psi}_{P, n}, \Pi_{P, n} W_{P, n} \check{\psi}_{P, n}\right\rangle+P-\lambda^{2} \bar{\int}_{\sigma_{n}}^{k} d^{3} k \frac{k}{2|k|^{3} \alpha_{P, n}^{2}(\hat{k})}, \\
\nabla E_{P, n-1} & =-\left\langle W_{P, n-1} \check{\psi}_{P, n-1}, \Pi_{P, n-1} W_{P, n-1} \check{\psi}_{P, n-1}\right\rangle+P-\lambda^{2} \int_{\sigma_{n-1}}^{k} d^{3} k \frac{k}{2|k|^{3} \alpha_{P, n-1}^{2}(\hat{k})} .
\end{aligned}
$$

Recalling that $\hat{\Pi}_{P, n}:=\widetilde{W}_{P, n} W_{P, n}^{*} \Pi_{P, n} W_{P, n} \widetilde{W}_{P, n}^{*}$, we can write

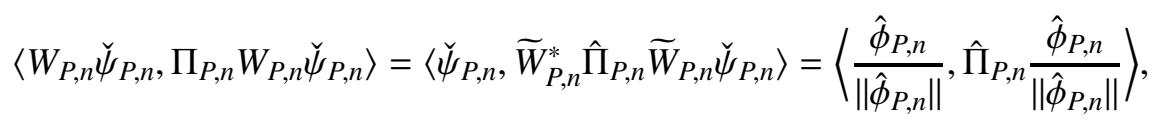

where we used $\hat{\phi}_{P, n} /\left\|\hat{\phi}_{P, n}\right\|=\widetilde{W}_{P, n} \breve{\psi}_{P, n}$. Denoting for any vector $\psi$ its normalized counterpart by $\psi^{*}:=\psi /\|\psi\|$, so that $\breve{\phi}_{P, n}=\phi_{P, n}^{*}$, we obtain

$$
\begin{aligned}
& \left\langle\hat{\phi}_{P, n}^{*}, \hat{\Pi}_{P, n} \hat{\phi}_{P, n}^{*}\right\rangle-\left\langle\phi_{P, n-1}^{*}, \Pi_{P, n-1} \phi_{P, n-1}^{*}\right\rangle \\
& =\left\langle\hat{\phi}_{P, n}^{*}, \hat{\Pi}_{P, n}\left(\hat{\phi}_{P, n}^{*}-\phi_{P, n-1}^{*}\right)\right\rangle+\left\langle\hat{\phi}_{P, n}^{*},\left(\hat{\Pi}_{P, n}-\Pi_{P, n-1}\right) \phi_{P, n-1}^{*}\right\rangle+\left\langle\left(\hat{\phi}_{P, n}^{*}-\phi_{P, n-1}^{*}\right), \Pi_{P, n-1} \phi_{P, n-1}^{*}\right\rangle .
\end{aligned}
$$

Now we recall formula (3.31)

$$
\hat{\Pi}_{P, n}-\Pi_{P, n-1}=\left.\mathcal{L}\right|_{n} ^{n-1}+\lambda^{2} \bar{\int}_{\sigma_{n}}^{\sigma_{n-1}} d^{3} k \frac{k\left(\alpha_{P, n}^{2}(\hat{k})-\alpha_{P, n-1}^{2}(\hat{k})\right)}{2|k|^{3} \alpha_{P, n-1}^{2}(\hat{k}) \alpha_{P, n}^{2}(\hat{k})}
$$

and we get

$$
\begin{aligned}
\nabla E_{P, n-1}-\nabla E_{P, n}= & \left\langle\hat{\phi}_{P, n}^{*}, \hat{\Pi}_{P, n}\left(\hat{\phi}_{P, n}^{*}-\phi_{P, n-1}^{*}\right)\right\rangle+\left\langle\left(\hat{\phi}_{P, n}^{*}-\phi_{P, n-1}^{*}\right), \Pi_{P, n-1} \phi_{P, n-1}^{*}\right\rangle \\
& +\left\langle\hat{\phi}_{P, n}^{*}, \phi_{P, n-1}^{*}\right\rangle \lambda^{2} \bar{\int}_{\sigma_{n}}^{\sigma_{n-1}} d^{3} k \frac{k\left(\alpha_{P, n}^{2}(\hat{k})-\alpha_{P, n-1}^{2}(\hat{k})\right)}{2|k|^{3} \alpha_{P, n-1}^{2}(\hat{k}) \alpha_{P, n}^{2}(\hat{k})} \\
& +\left\langle\hat{\phi}_{P, n}^{*},\left.\mathcal{L}\right|_{n} ^{n-1} \phi_{P, n-1}^{*}\right\rangle-\lambda^{2} \bar{\int}_{\sigma_{n-1}}^{K} d^{3} k \frac{k\left(\alpha_{P, n}^{2}(\hat{k})-\alpha_{P, n-1}^{2}(\hat{k})\right)}{2|k|^{3} \alpha_{P, n-1}^{2}(\hat{k}) \alpha_{P, n}^{2}(\hat{k})} \\
& +\lambda^{2} \bar{\int}_{\sigma_{n}}^{\sigma_{n-1}} d^{3} k \frac{k}{2|k|^{3} \alpha_{P, n}^{2}(\hat{k})} .
\end{aligned}
$$

Consequently,

$$
\begin{aligned}
\nabla E_{P, n-1}-\nabla E_{P, n}+ & \lambda^{2} \int_{\sigma_{n-1}}^{k} d^{3} k \frac{k\left(-\hat{k} \cdot \nabla E_{P, n}+\hat{k} \cdot \nabla E_{P, n-1}\right)\left(2-\hat{k} \cdot \nabla E_{P, n}-\hat{k} \cdot \nabla E_{P, n-1}\right)}{2|k|^{3} \alpha_{P, n-1}^{2}(\hat{k}) \alpha_{P, n}^{2}(\hat{k})} \\
= & \left\langle\hat{\phi}_{P, n}^{*}, \phi_{P, n-1}^{*}\right\rangle \lambda^{2} \bar{\sigma}_{\sigma_{n}}^{\sigma_{n-1}} d^{3} k \frac{k\left(\alpha_{P, n}^{2}(\hat{k})-\alpha_{P, n-1}^{2}(\hat{k})\right)}{2|k|^{3} \alpha_{P, n-1}^{2}(\hat{k}) \alpha_{P, n}^{2}(\hat{k})} \\
& +\left\langle\hat{\phi}_{P, n}^{*}, \hat{\Pi}_{P, n}\left(\hat{\phi}_{P, n}^{*}-\phi_{P, n-1}^{*}\right)\right\rangle+\left\langle\left(\hat{\phi}_{P, n}^{*}-\phi_{P, n-1}^{*}\right), \Pi_{P, n-1} \phi_{P, n-1}^{*}\right\rangle \\
& +\left\langle\hat{\phi}_{P, n}^{*},\left.\mathcal{L}\right|_{n} ^{n-1} \phi_{P, n-1}^{*}\right\rangle+\lambda^{2} \int_{\sigma_{n}}^{\sigma_{n-1}} d^{3} k \frac{k}{2|k|^{3} \alpha_{P, n}^{2}(\hat{k})} .
\end{aligned}
$$


To conclude, we have to analyze the term involving $\left.\mathcal{L}\right|_{n} ^{n-1}$. We recall that

$$
\left.\mathcal{L}\right|_{n} ^{n-1}:=-\lambda \int_{\sigma_{n}}^{\sigma_{n-1}} d^{3} k \frac{k\left(b(k)+b^{*}(k)\right)}{\sqrt{2}|k|^{3 / 2} \alpha_{P, n-1}(\hat{k})}
$$

and denote by $\left(\left.\mathcal{L}\right|_{n} ^{n-1}\right)^{( \pm)}$its creation (+) and annihilation (-) parts. We note that $\left(\left.\mathcal{L}\right|_{n} ^{n-1}\right)^{(-)} \phi_{P, n-1}^{*}=0$, since $\phi_{P, n-1}^{*} \in \mathcal{F}_{n-1}$, thus it suffices to study $\left\langle\hat{\phi}_{P, n}^{*},\left(\left.\mathcal{L}\right|_{n} ^{n-1}\right)^{(+)} \phi_{P, n-1}^{*}\right\rangle$. We write

$$
\begin{aligned}
\left(\left.\mathcal{L}\right|_{n} ^{n-1}\right)^{(-)} \hat{\phi}_{P, n}^{*} & =\left(\left.\mathcal{L}\right|_{n} ^{n-1}\right)^{(-)} \widetilde{W}_{P, n} W_{P, n}^{*} \phi_{P, n}^{*}=\left(\left.\mathcal{L}\right|_{n} ^{n-1}\right)^{(-)} \widetilde{W}_{P, n} \breve{\psi}_{P, n} \\
& =\widetilde{W}_{P, n}\left\{\left(\left.\mathcal{L}\right|_{n} ^{n-1}\right)^{(-)}-\lambda^{2} \bar{\sigma}_{\sigma_{n}}^{\sigma_{n-1}} d^{3} k \frac{k}{2|k|^{3} \alpha_{P, n-1}^{2}(\hat{k})}\right) \breve{\psi}_{P, n} \\
& =\widetilde{W}_{P, n}\left(\left.\mathcal{L}\right|_{n} ^{n-1}\right)^{(-)} \breve{\psi}_{P, n}+O\left(\lambda^{2} \sigma_{n-1}\right) .
\end{aligned}
$$

Now we estimate using Lemmas D.2, D.3,

$$
\left(\left.\mathcal{L}\right|_{n} ^{n-1}\right)^{(-)} \check{\psi}_{P, n}=-\lambda^{2} \bar{\int}_{\sigma_{n}}^{\sigma_{n-1}} d^{3} k \frac{k}{2|k|^{2} \alpha_{P, n-1}(\hat{k})} \frac{1}{E_{P, n}-|k|-H_{P-k, n}} \breve{\psi}_{P, n}=O\left(\lambda^{2} \sigma_{n-1}\right) .
$$

Now making use of (D.93), (D.92) and (D.90), and exploiting the fact that $\left\|\hat{\Pi}_{P, n} \phi_{P, n}^{*}\right\|,\left\|\Pi_{P, n-1} \phi_{P, n-1}^{*}\right\|<\infty$ (which follows from (D.83)) together with $\left|\nabla E_{P, n}\right| \leq c$, we conclude the proof.

\section{E Convexity of the ground state energy}

The discussion in this appendix is similar to [23] but the proof is streamlined using estimates from the present paper.

\section{E.1 Main line of the argument}

In order to show the strict convexity of $E_{P}:=\lim _{n \rightarrow \infty} E_{P, n}$ it is convenient to first state a result concerning $\partial_{|P|}^{2} E_{P, n}$.

Lemma E.1. Under the assumptions of Theorem 4.3

$$
\left|\partial_{|P|}^{2} E_{P, n}-\partial_{|P|}^{2} E_{P, n-1}\right| \leq c|\lambda|^{1 / 8} \sigma_{n-1}^{1-2 \delta} .
$$

Proof. Starting from formula (5.51), coming back to the discrete cut-off and exploiting rotation invariance of the model, we can write

$$
\partial_{|P|}^{2} E_{P, n}=1-\left.2\left\langle\check{\phi}_{P, n},\left(\Gamma_{P, n}\right)_{i} \frac{1}{H_{P, n}^{W}-E_{P, n}}\left(\Gamma_{P, n}\right)_{i} \check{\phi}_{P, n}\right\rangle\right|_{P=|P| \hat{P}_{i}},
$$

where $\hat{P}_{i}$ is the unit vector in the $i$-th direction for some fixed $i$. In the remaining part of this proof it is tacitly assumed that $P=|P| \hat{P}_{i}$. Now we define the function

$$
\begin{aligned}
f_{n}(z):= & \left\langle\check{\phi}_{P, n},\left(\Gamma_{P, n}\right)_{i} \frac{1}{H_{P, n}^{W}-z}\left(\Gamma_{P, n}\right)_{i} \check{\phi}_{P, n}\right\rangle \\
& -\left\langle\check{\phi}_{P, n-1},\left(\Gamma_{P, n-1}\right)_{i} \frac{1}{H_{P, n-1}^{W+}-z+\left(E_{P, n}-E_{P, n-1}\right)-\left.\Delta c_{P}\right|_{n} ^{n-1}}\left(\Gamma_{P, n-1}\right)_{i} \check{\phi}_{P, n-1}\right\rangle .
\end{aligned}
$$


Making use of the maximal modulus principle, applied to this function, and the fact that $\left\langle\breve{\phi}_{P, n},\left(\Gamma_{P, n}\right)_{i} \check{\phi}_{P, n}\right\rangle=0$, we can write

$$
\begin{aligned}
& \left|\partial_{|P|}^{2} E_{P, n}-\partial_{|P|}^{2} E_{P, n-1}\right| \\
& \leq \sup _{z_{n+1} \in \tilde{\gamma}_{P, n+1}} 2\left|\left\langle\check{\phi}_{P, n},\left(\Gamma_{P, n}\right)_{i} \frac{1}{H_{P, n}^{W}-z_{n+1}}\left(\Gamma_{P, n}\right)_{i} \check{\phi}_{P, n}\right\rangle-\left\langle\check{\phi}_{P, n-1},\left(\Gamma_{P, n-1}\right)_{i} \frac{1}{H_{P, n-1}^{W+}-z_{n+1}}\left(\Gamma_{P, n-1}\right)_{i} \check{\phi}_{P, n-1}\right\rangle\right| \\
& \quad+O\left(|\lambda|^{2} \sigma_{n-1}^{1-2 \delta}\right),
\end{aligned}
$$

where the error term (E.5) comes from the shift of the resolvent in (E.3), Theorem 4.1 and the bounds $\mid E_{P, n-1}-$ $\left.\left.E_{P, n}|,| \Delta c_{P}\right|_{n} ^{n-1}|\leq c| \lambda\right|^{2} \sigma_{n-1}$. Now we rearrange the first term under the absolute value in (E.4):

$$
F\left(z_{n+1}\right):=\left\langle\check{\phi}_{P, n},\left(\Gamma_{P, n}\right)_{i} \frac{1}{H_{P, n}^{W}-z_{n+1}}\left(\Gamma_{P, n}\right)_{i} \check{\phi}_{P, n}\right\rangle=\left\langle\hat{\phi}_{P, n}^{*},\left(\hat{\Gamma}_{P, n}\right)_{i} \frac{1}{\hat{H}_{P, n}^{W}-z_{n+1}}\left(\hat{\Gamma}_{P, n}\right)_{i} \hat{\phi}_{P, n}^{*}\right\rangle,
$$

where $\hat{\phi}_{P, n}^{*}:=\hat{\phi}_{P, n} /\left\|\hat{\phi}_{P, n}\right\|$. Furthermore, we write

$$
\begin{aligned}
F\left(z_{n+1}\right)= & \left\langle\left(\hat{\phi}_{P, n}^{*}-\check{\phi}_{P, n-1}\right),\left(\hat{\Gamma}_{P, n}\right)_{i} \frac{1}{\hat{H}_{P, n}^{W}-z_{n+1}}\left(\hat{\Gamma}_{P, n}\right)_{i} \hat{\phi}_{P, n}^{*}\right\rangle \\
& +\left\langle\check{\phi}_{P, n-1},\left(\hat{\Gamma}_{P, n}\right)_{i} \frac{1}{\hat{H}_{P, n}^{W}-z_{n+1}}\left(\hat{\Gamma}_{P, n}\right)_{i}\left(\hat{\phi}_{P, n}^{*}-\check{\phi}_{P, n-1}\right)\right\rangle \\
& +\left\langle\check{\phi}_{P, n-1},\left(\hat{\Gamma}_{P, n}\right)_{i} \frac{1}{\hat{H}_{P, n}^{W}-z_{n+1}}\left(\hat{\Gamma}_{P, n}\right)_{i} \check{\phi}_{P, n-1}\right\rangle .
\end{aligned}
$$

Using Lemmas E.4, E.5 and the estimate $\left\|\hat{\phi}_{P, n}^{*}-\breve{\phi}_{P, n-1}\right\| \leq 4|\lambda|^{1 / 4} \sigma_{n-1}^{1-\delta}$ (which follows from Theorem 4.1) we can write

$$
(E .7)+(E .8)=O\left(|\lambda|^{1 / 8} \sigma_{n-1}^{1-2 \delta}\right),
$$

where we also exploited $|\lambda| \leq \epsilon^{8}$. As for (E.9), we have

$$
\begin{aligned}
(E .9)= & \left\langle\check{\phi}_{P, n-1},\left(\left.\Delta \Gamma_{P}\right|_{n} ^{n-1}\right)_{i} \frac{1}{\hat{H}_{P, n}^{W}-z_{n+1}}\left(\left.\Delta \Gamma_{P}\right|_{n} ^{n-1}\right)_{i} \check{\phi}_{P, n-1}\right\rangle \\
& +\left\langle\check{\phi}_{P, n-1},\left(\left.\Delta \Gamma_{P}\right|_{n} ^{n-1}\right)_{i} \frac{1}{\hat{H}_{P, n}^{W}-z_{n+1}}\left(\Gamma_{P, n-1}\right)_{i} \check{\phi}_{P, n-1}\right\rangle \\
& +\left\langle\check{\phi}_{P, n-1},\left(\Gamma_{P, n-1}\right)_{i} \frac{1}{\hat{H}_{P, n}^{W}-z_{n+1}}\left(\left.\Delta \Gamma_{P}\right|_{n} ^{n-1}\right)_{i} \check{\phi}_{P, n-1}\right\rangle \\
& +\left\langle\check{\phi}_{P, n-1},\left(\Gamma_{P, n-1}\right)_{i} \frac{1}{\hat{H}_{P, n}^{W}-z_{n+1}}\left(\Gamma_{P, n-1}\right)_{i} \check{\phi}_{P, n-1}\right\rangle,
\end{aligned}
$$

where $\left.\Delta \Gamma_{P}\right|_{n} ^{n-1}$ is defined in (4.77). Making use of Lemma E.6 and of $\left\|\left(\hat{H}_{P, n}^{W}-z_{n+1}\right)^{-1}\right\|_{\mathcal{F}_{n}}=O\left(\sigma_{n+1}^{-1}\right)$, we obtain

$$
(E .11)+(E .12)+(E .13)=O\left(|\lambda|^{1 / 4} \sigma_{n-1}^{1-2 \delta}\right)
$$

where we also used $|\lambda| \leq \epsilon^{8}$.

Thus coming back to (E.4) and making use of the expansion in (3.25) we can write

$$
\begin{aligned}
& \left|\partial_{|P|}^{2} E_{P, n}-\partial_{|P|}^{2} E_{P, n-1}\right| \\
& \leq \sup _{z_{n+1} \in \tilde{\gamma}_{P, n+1}} 2\left|\left\langle\check{\phi}_{P, n-1},\left(\Gamma_{P, n-1}\right)_{i} \sum_{j=1}^{\infty}\left\{\frac{1}{H_{P, n-1}^{W+}-z_{n+1}}\left(-\left.H_{I}^{W}\right|_{n} ^{n-1}\right)\right\}^{j} \frac{1}{H_{P, n-1}^{W+}-z_{n+1}}\left(\Gamma_{P, n-1}\right)_{i} \check{\phi}_{P, n-1}\right\rangle\right| \\
& \quad+O\left(|\lambda|^{1 / 8} \sigma_{n-1}^{1-2 \delta}\right) .
\end{aligned}
$$


Adopting the notation of (4.16), (4.17), the term under the modulus in (E.16) can be rewritten as follows

$$
\begin{aligned}
\left\langle\check{\phi}_{P, n-1},\left(\Gamma_{P, n-1}\right)_{i} \sum_{j=1}^{\infty}\{R V\}^{j} R\left(\Gamma_{P, n-1}\right)_{i} \check{\phi}_{P, n-1}\right\rangle= & \left\langle\check{\phi}_{P, n-1},\left(\Gamma_{P, n-1}\right)_{i} \sum_{j=0}^{\infty}\{R V\}^{j} R V R\left(\Gamma_{P, n-1}\right)_{i} \check{\phi}_{P, n-1}\right\rangle \\
= & \left\langle\check{\phi}_{P, n-1},\left(\Gamma_{P, n-1}\right)_{i} \sum_{j=0}^{\infty}\{R V\}^{j} Q_{P, n-1}^{\perp} R V R\left(\Gamma_{P, n-1}\right)_{i} \check{\phi}_{P, n-1}\right\rangle \\
& +\left\langle\check{\phi}_{P, n-1},\left(\Gamma_{P, n-1}\right)_{i} \sum_{j=0}^{\infty}\{R V\}^{j} R V Q_{P, n-1} R V R\left(\Gamma_{P, n-1}\right)_{i} \check{\phi}_{P, n-1}\right\rangle,
\end{aligned}
$$

where in the last step we used $\left\langle\check{\phi}_{P, n-1},\left(\Gamma_{P, n-1}\right)_{i} \check{\phi}_{P, n-1}\right\rangle=0$. We recall that $\left\|\sum_{j=0}^{\infty}\{R V\}^{j}\right\|_{\mathcal{F}_{n}}=O(1)$ by Lemma 3.12 and $\left\|\Gamma_{P, n-1} \check{\phi}_{P, n-1}\right\|=O(1)$. Now making use of Lemma 4.18 we write

$$
\begin{aligned}
(E .18) & =\left\langle\check{\phi}_{P, n-1},\left(\Gamma_{P, n-1}\right)_{i} \sum_{j=1}^{\infty}\{R V\}^{j} b^{*}\left(\left.g_{i}\right|_{n} ^{n-1}\right) \phi_{P, n-1}\right\rangle+O\left(|\lambda|^{1 / 2} \sigma_{n-1}^{1-2 \delta}\right) \\
& =\left\langle\check{\phi}_{P, n-1},\left(\Gamma_{P, n-1}\right)_{i} \sum_{j=0}^{\infty}\{R V\}^{j} R V b^{*}\left(\left.g_{i}\right|_{n} ^{n-1}\right) \phi_{P, n-1}\right\rangle+O\left(|\lambda|^{1 / 2} \sigma_{n-1}^{1-2 \delta}\right)=O\left(|\lambda|^{1 / 2} \sigma_{n-1}^{1-2 \delta}\right),
\end{aligned}
$$

where in the second step we made use of the fact that $b\left(\left.g_{i}\right|_{n} ^{n-1}\right)\left(\Gamma_{P, n-1}\right)_{i} \breve{\phi}_{P, n-1}=0$ and in the last step we applied Lemma 4.19. Furthermore, we get by Lemma 4.10, estimate (4.113) and Theorem 4.1

$$
(E .19)=O\left(|\lambda| \sigma_{n-1}^{1-2 \delta}\right) .
$$

Thus altogether we obtain the bound in (E.1).

Theorem E.2. Assume $\left|\lambda_{0}\right|$ sufficiently small and $|P|<P_{\max }$. Then $\partial_{|P|}^{2} E_{P, n}$ defined in (E.2) is strictly positive for any $n \in \mathbb{N}_{0}$ and converges to a limiting function $\mathcal{E}_{\lambda}^{\prime \prime}(|P|)>0$ as $n \rightarrow \infty$. The function $\mathcal{E}_{\lambda}^{\prime \prime}(|P|)$ is Hölder continuous for some exponent $\eta(>0)$ and $\lim _{\lambda \rightarrow 0} \mathcal{E}_{\lambda}^{\prime \prime}(|P|)=1$.

Proof. First, we note that the estimate in (E.1) yields the existence of $\lim _{n \rightarrow \infty} \partial_{|P|}^{2} E_{P, n}<\infty$ by a telescopic argument. Next, we observe that $\partial_{|P|}^{2} E_{P, n=0}=1$ (see (E.2)), because $\left(\Gamma_{P, n=0}\right)_{i} \equiv\left(P_{\mathrm{f}}\right)_{i}$ and $\phi_{P, n=0} \equiv \Omega$. Consequently, again by a telescopic argument,

$$
\left.2\left|\left\langle\check{\phi}_{P, n},\left(\Gamma_{P, n}\right)_{i} \frac{1}{H_{P, n}^{W}-E_{P, n}}\left(\Gamma_{P, n}\right)_{i} \check{\phi}_{P, n}\right\rangle\right|_{P=|P| \hat{P}_{i}}|\leq c| \lambda\right|^{1 / 8},
$$

which implies that $\partial_{|P|}^{2} E_{P, n}>0$ and $\mathcal{E}_{\lambda}^{\prime \prime}(|P|)>0$ for $P \in S$ and $\lambda_{0}$ sufficiently small. This also gives $\lim _{\lambda \rightarrow 0} \mathcal{E}_{\lambda}^{\prime \prime}(|P|)=1$.

Starting from the expression in (E.2) and the convergence $\lim _{n \rightarrow \infty} \partial_{|P|}^{2} E_{P, n}=\mathcal{E}^{\prime \prime}(|P|)$, a standard argument ensures that $\mathcal{E}^{\prime \prime}(|P|)$ is Hölder continuous for some small exponent. Indeed, for $P, P+\Delta P \in S$ it is enough to write

$$
\begin{aligned}
& \mathcal{E}^{\prime \prime}(|P+\Delta P|)-\mathcal{E}^{\prime \prime}(|P|) \\
= & \mathcal{E}^{\prime \prime}(|P+\Delta P|)-\partial_{|P|}^{2} E_{P+\Delta P, n_{*}}+\partial_{|P|}^{2} E_{P+\Delta P, n_{*}}-\partial_{|P|}^{2} E_{P, n_{*}}+\partial_{|P|}^{2} E_{P, n_{*}}-\mathcal{E}^{\prime \prime}(|P|),
\end{aligned}
$$

where the infrared cut-off is $\epsilon^{n_{*}}=O\left(|\Delta P|^{\frac{1}{4}}\right)$ and we exploit the convergence rate of $\partial_{|P|}^{2} E_{P, n} \rightarrow \mathcal{E}^{\prime \prime}(|P|)$, the estimate on the gap of $H_{P, n}^{W} 1 \mathcal{F}_{n}$, and the fact that $\left\{H_{P, n}^{W}\right\}_{P \in S}$ is an analytic family of type A. (Alternatively, one can use our bound $\left|\partial_{P}^{\beta_{3}} E_{P, \sigma}\right| \leq c / \sigma^{\delta_{\lambda_{0}}}$ and the Taylor theorem to estimate $\left.\partial_{|P|}^{2} E_{P+\Delta P, n_{*}}-\partial_{|P|}^{2} E_{P, n_{*}}\right)$. 
Corollary E.3. Under the assumptions of Theorem E.2, the limiting function $E_{P}:=\lim _{n \rightarrow \infty} E_{P, n}$ is twice continuously differentiable and $\partial_{|P|}^{2} E_{P} \equiv \mathcal{E}_{\lambda}^{\prime \prime}(|P|)$.

Proof. Clearly, there exists $C$ such that $\left|\partial_{|P|} E_{P, n}\right|,\left|\partial_{|P|}^{2} E_{P, n}\right|<C$ for all $P \in S:=\left\{P \in \mathbb{R}^{3}|| P \mid<P_{\max }=\frac{1}{3}\right\}$ and for all $n \in \mathbb{N}_{0}$. Thus we can write for any $P, P+\Delta P$ in $S$

$$
\begin{aligned}
& E_{P+\Delta P}-E_{P}=\lim _{n \rightarrow \infty}\left\{E_{P+\Delta P, n}-E_{P, n}\right\}=\lim _{n \rightarrow \infty} \int_{0}^{1} \Delta P \cdot\left\{\frac{Q}{|Q|} \partial_{|Q|} E_{Q, n}\right\}_{Q=P+u \Delta P} d u \\
= & \int_{0}^{1} \lim _{n \rightarrow \infty}\left\{\Delta P \cdot\left\{\frac{Q}{|Q|} \partial_{|Q|} E_{Q, n}\right\}_{Q=P+u \Delta P}\right\} d u .
\end{aligned}
$$

The Hölder continuity of $\lim _{n \rightarrow \infty} \partial_{|P|} E_{P, n}$ is shown as in (E.23), exploiting (3.19) and Theorem 4.1 instead of (E.1). By this Hölder continuity and the fundamental theorem of calculus we conclude that $E_{P}$ is differentiable and $\nabla E_{P}=\lim _{n \rightarrow \infty} \partial_{|P|} E_{P, n} \frac{P}{|P|}$. An analogous argument implies that the second derivatives of $E_{P}$ exist with $\partial_{|P|}^{2} E_{P} \equiv \mathcal{E}_{\lambda}^{\prime \prime}(|P|)$.

\section{E.2 Auxiliary lemmas}

Lemma E.4. Under the assumptions of Theorem 4.3

$$
\left\|\left(\hat{\Gamma}_{P, n}\right)_{i} \frac{1}{\hat{H}_{P, n}^{W}-z_{n+1}}\left(\hat{\Gamma}_{P, n}\right)_{i} \hat{\phi}_{P, n}^{*}\right\|=O\left(\sigma_{n}^{-\delta}\right) .
$$

Proof. Clearly we can drop the 'hats' and write

$$
\begin{aligned}
\left\|\left(\Gamma_{P, n}\right)_{i} \frac{1}{H_{P, n}^{W}-z_{n+1}}\left(\Gamma_{P, n}\right)_{i} \check{\phi}_{P, n}\right\| \leq & \left\|\left(\Gamma_{P, n}\right)_{i} \chi\left(H_{P, n}^{W}-E_{P, n} \leq 1\right) \frac{1}{H_{P, n}^{W}-z_{n+1}}\left(\Gamma_{P, n}\right)_{i} \check{\phi}_{P, n}\right\| \\
& +\left\|\left(\Gamma_{P, n}\right)_{i} \chi\left(H_{P, n}^{W}-E_{P, n} \geq 1\right) \frac{1}{H_{P, n}^{W}-z_{n+1}}\left(\Gamma_{P, n}\right)_{i} \check{\phi}_{P, n}\right\|,
\end{aligned}
$$

where we inserted spectral projections of $H_{P, n}^{W}$. Since $\left(\Gamma_{P, n}\right)_{i} \chi\left(H_{P, n}^{W}-E_{P, n} \leq 1\right)$ is bounded, uniformly in $n$, we have by Theorem 4.1 that $(E .27)=O\left(\sigma_{n}^{-\delta}\right)$.

As for (E.28), we note that $\chi\left(H_{P, n}^{W}-E_{P, n} \geq 1\right)$ mollifies the infrared singularity of the resolvent. Hence, making use of

$$
\left\|\chi\left(H_{P, n}^{W}-E_{P, n} \geq 1\right)\left(i+H_{P, n}^{W}\right)^{\frac{1}{2}} \frac{1}{H_{P, n}^{W}-z_{n+1}}\right\|=O(1), \quad\left\|\left(\Gamma_{P, n}\right)_{i}\left(i+H_{P, n}^{W}\right)^{-1 / 2}\right\|=O(1),
$$

and of $\left\|\left(\Gamma_{P, n}\right)_{i} \check{\phi}_{P, n}\right\|=O(1)$ we obtain $(E .28)=O(1)$ and conclude the proof.

Lemma E.5. Under the assumptions of Theorem 4.3

$$
\left\|\left(\hat{\Gamma}_{P, n}\right)_{i} \frac{1}{\hat{H}_{P, n}^{W}-z_{n+1}}\left(\hat{\Gamma}_{P, n}\right)_{i} \check{\phi}_{P, n-1}\right\|=O\left(\sigma_{n}^{-\delta}\right)
$$

Proof. We write

$$
\begin{aligned}
\left\|\left(\hat{\Gamma}_{P, n}\right)_{i} \frac{1}{\hat{H}_{P, n}^{W}-z_{n+1}}\left(\hat{\Gamma}_{P, n}\right)_{i} \check{\phi}_{P, n-1}\right\|= & \left\|\left(\Gamma_{P, n}\right)_{i} \frac{1}{H_{P, n}^{W}-z_{n+1}}\left(\Gamma_{P, n}\right)_{i} W_{P, n} \widetilde{W}_{P, n}^{*} \check{\phi}_{P, n-1}\right\| \\
\leq & \left\|\left(\Gamma_{P, n}\right)_{i} \frac{1}{H_{P, n}^{W}-z_{n+1}}\left(\Gamma_{P, n}\right)_{i} \check{\phi}_{P, n}\right\| \\
& +\left\|\left(\Gamma_{P, n}\right)_{i} \frac{1}{H_{P, n}^{W}-z_{n+1}}\left(\Gamma_{P, n}\right)_{i}\right\|_{\mathcal{F}_{n}}\left\|W_{P, n} \widetilde{W}_{P, n}^{*} \check{\phi}_{P, n-1}-\check{\phi}_{P, n}\right\| .
\end{aligned}
$$


We immediately get from Lemma E.4 that $(E .31)=O\left(\sigma_{n}^{-\delta}\right)$. Furthermore, we note that by (3.18) and Theorem 4.1

$$
\left\|W_{P, n} \widetilde{W}_{P, n}^{*} \check{\phi}_{P, n-1}-\check{\phi}_{P, n}\right\|=\left\|\check{\phi}_{P, n-1}-\hat{\phi}_{P, n}^{*}\right\| \leq 4|\lambda|^{1 / 4} \sigma_{n-1}^{1-\delta} .
$$

Finally, we estimate similarly as in (E.27), (E.28)

$$
\begin{aligned}
\left\|\left(\Gamma_{P, n}\right)_{i} \frac{1}{H_{P, n}^{W}-z_{n+1}}\left(\Gamma_{P, n}\right)_{i}\right\|_{\mathcal{F}_{n} \leq} \leq & \left\|\left(\Gamma_{P, n}\right)_{i} \chi\left(H_{P, n}^{W}-E_{P, n} \leq 1\right) \frac{1}{H_{P, n}^{W}-z_{n+1}}\left(\Gamma_{P, n}\right)_{i}\right\|_{\mathcal{F}_{n}} \\
& +\left\|\left(\Gamma_{P, n}\right)_{i} \chi\left(H_{P, n}^{W}-E_{P, n} \geq 1\right) \frac{1}{H_{P, n}^{W}-z_{n+1}}\left(\Gamma_{P, n}\right)_{i}\right\|_{\mathcal{F}_{n}} \\
= & O\left(\sigma_{n+1}^{-1}\right)
\end{aligned}
$$

where we made use of $\left\|\left(\Gamma_{P, n}\right)_{i} \chi\left(H_{P, n}^{W}-E_{P, n} \leq 1\right)\right\|_{\mathcal{F}_{n}}=O(1)$ and $\left\|\left(H_{P, n}^{W}-z_{n+1}\right)^{-1}\right\|_{\mathcal{F}_{n}}=O\left(\sigma_{n+1}^{-1}\right)$ to estimate (E.34) and applied (E.29) to estimate (E.35). From (E.36) and (E.33) we obtain $(E .32)=O\left(\sigma_{n-1}^{-\delta}\right)$, where we also exploited $|\lambda| \leq \epsilon^{8}$. This concludes the proof.

Lemma E.6. Under the assumptions of Theorem 4.3

$$
\begin{aligned}
& \left(\left.\Delta \Gamma_{P}\right|_{n} ^{n-1}\right)_{i} \check{\phi}_{P, n-1}=O\left(|\lambda|^{1 / 4} \sigma_{n-1}^{1-\delta}\right), \\
& \frac{1}{\hat{H}_{P, n}^{W}-z_{n+1}}\left(\Gamma_{P, n-1}\right)_{i} \check{\phi}_{P, n-1}=O\left(\sigma_{n-1}^{-\delta}\right) .
\end{aligned}
$$

Proof. As for (E.37), we recall from (4.77) that $\left.\Delta \Gamma_{P}\right|_{n} ^{n-1}=\left.X\right|_{n} ^{n-1}+\left.\mathcal{L}\right|_{n} ^{n-1}$, where $\left.X\right|_{n} ^{n-1}:=-\nabla E_{P, n-1}+\nabla E_{P, n}+\left.\mathcal{I}\right|_{n} ^{n-1}$ is a vector in $\mathbb{R}^{3}$ satisfying

$$
\left.|X|_{n}^{n-1}|\leq c| \lambda\right|^{1 / 4} \sigma_{n-1}^{1-\delta}
$$

Thus it suffices to use that

$$
\left.\mathcal{L}\right|_{n} ^{n-1} \check{\phi}_{P, n-1}=\left(\left.\mathcal{L}\right|_{n} ^{n-1}\right)^{(+)} \check{\phi}_{P, n-1}=O\left(|\lambda| \sigma_{n-1}\right),
$$

which follows from the definition of $\left.\mathcal{L}\right|_{n} ^{n-1}$.

To prove (E.38), we write, making use of the expansion (3.25) and Theorem 4.1

$$
\frac{1}{\hat{H}_{P, n}^{W}-z_{n+1}}\left(\Gamma_{P, n-1}\right)_{i} \check{\phi}_{P, n-1}=\sum_{j=0}^{\infty}\left\{\frac{1}{H_{P, n-1}^{W+}-z_{n+1}}\left(-\left.H_{I}^{W}\right|_{n} ^{n-1}\right)\right\}^{j} \frac{1}{H_{P, n-1}^{W+}-z_{n+1}}\left(\Gamma_{P, n-1}\right)_{i} \check{\phi}_{P, n-1}=O\left(\sigma_{n-1}^{-\delta}\right),
$$

where we controlled the sum as explained below (E.19).

\section{References}

[1] A. Abdesselam and D. Hasler, Analyticity of the ground state energy for massless Nelson models. Commun. Math. Phys. 310, (2012) 511-536.

[2] S. Albeverio, Scattering theory in a model of quantum fields. J. Math. Phys. 14, (1973) 1800-1816.

[3] V. Bach, M. Ballesteros and A.Pizzo, Existence and construction of resonances for atoms coupled to the quantized radiation field. Advances in Mathematics 314, (2017) 540-572. 
[4] V. Bach, T. Chen, J. Fröhlich, and I. M. Sigal, Effective Dynamics of an Electron Coupled to an External Potential in Non-relativistic QED. Ann. Henri Poincaré 6, (2013) 1573-1597.

[5] V. Bach, T. Chen, J. Fröhlich, and I. M. Sigal, The renormalized electron mass in non-relativistic quantum electrodynamics. J. Funct. Anal. 243, (2007) 426-535.

[6] S. Bachmann, D.-A. Deckert and A. Pizzo, The mass shell of the Nelson model without cut-offs. J. Funct. Anal. 263, (2012) 1224-1282.

[7] T. Chen, J. Fröhlich and A. Pizzo, Infraparticle scattering states in non-relativistic QED: I. The BlochNordsieck paradigm. Commun. Math. Phys. 294, (2010) 761-825.

[8] T. Chen, J. Fröhlich and A. Pizzo, Infraparticle scattering states in non-relativistic QED: II. Mass shell properties. J. Math. Phys. 50, (2009) 012103-012134.

[9] J. Dereziński and C. Gérard, Scattering theory of infrared divergent Pauli-Fierz Hamiltonians. Ann. Henri Poincaré 5, (2004) 523-577.

[10] W. De Roeck and A. Kupiainen, Approach to ground state and time-independent photon bound for massless spin-boson models. Ann. Henri Poincaré 14, (2013) 253-311.

[11] W. Dybalski, Haag-Ruelle scattering theory in presence of massless particles. Lett. Math. Phys. 72 , (2005) 27-38.

[12] W. Dybalski, From Faddeev-Kulish to LSZ. Towards a non-perturbative description of colliding electrons. Nuclear Physics B 925, (2017) 455-469.

[13] W. Dybalski and J.S. Møller, The translation invariant massive Nelson model: III. Asymptotic completeness below the two-boson threshold. Ann. Henri Poincaré 16, (2015) 2603-2693.

[14] W. Dybalski and A. Pizzo, Coulomb scattering in the massless Nelson model I. Foundations of twoelectron scattering. J. Stat. Phys. 154, (2014) 543-587.

[15] W. Dybalski and A. Pizzo, Coulomb scattering in the massless Nelson model III. Ground state wave functions and non-commutative recurrence relations. Ann. Henri Poincaré 19, (2018) 463-514.

[16] J. Faupin, J. Fröhlich and B. Schubnel, Analyticity of the self-energy in the total momentum of an atom coupled to the quantized radiation field. J. Funct. Anal. 267, (2014) 4139-4196.

[17] J. Fröhlich, On the infrared problem in a model of scalar electrons and massless, scalar bosons. Ann. Inst. H. Poincaré Sect. A (N.S.) 19, (1973) 1-103.

[18] J. Fröhlich, unpublished notes.

[19] J. Fröhlich, Existence of dressed one electron states in a class of persistent models. Fortschr. Phys. 22, (1974) 158-198.

[20] J. Fröhlich, Application of commutator theorems to the integration o representations of Lie algebras and commutation relations. Commun. Math. Phys. 54, (1977) 135-150.

[21] J. Fröhlich, M Griesemer and B. Schlein, Asymptotic electromagnetic fields in models of quantummechanical matter interacting with the quantized radiation field Advances in Mathematics 164, (2001) 349-398.

[22] J. Fröhlich, M. Griesemer and B. Schlein, Asymptotic completeness for Compton scattering. Commun. Math. Phys. 252, (2004) 415-476.

[23] J. Fröhlich and A. Pizzo, Renormalized electron mass in non-relativistic QED. Commun. Math. Phys. 294, (2010) 439-470. 
[24] M. Griesemer and D. Hasler, Analytic perturbation theory and renormalization analysis of matter coupled to quantized radiation. Ann. Henri Poincaré, 10 (2009) 577-621.

[25] E.P. Gross, Existence and uniqueness of physical ground states. J. Funct. Anal. 10, (1972) 52-109.

[26] R. Haag, Quantum field theories with composite particles and asymptotic conditions. Phys. Rev. 112, (1958) 669-673.

[27] M. Könenberg and O. Matte, The mass-shell in the semi-relativistic Pauli-Fierz model. Ann. Henri Poincaré 15, (2014) 863-915.

[28] O. Matte, On higher order estimates in quantum electrodynamics. Documenta Math. 15, (2010) 207234.

[29] A. Pizzo, One-particle (improper) states in Nelson's massless model. Ann. Henri Poincaré 4, (2003) 439-486.

[30] A. Pizzo, Scattering of an infraparticle: the one particle sector in Nelson's massless models, Ann. Henri Poincaré 4, (2005) 553-606.

[31] D. Ruelle, On the asymptotic condition in quantum field theory. Helv. Phys. Acta 35, (1962) 147-163.

[32] H. Spohn, Asymptotic completeness for Rayleigh scattering. J. Math. Phys. 38, (1997) 2281-2296. 